\title{
Pulmonary Large Cell Neuroendocrine Carcinoma
}

\author{
Citation for published version (APA):
}

Hermans, B. C. M. (2021). Pulmonary Large Cell Neuroendocrine Carcinoma: a unique type of lung cancer? Identification of molecular and clinical subtypes \& consequences for treatment. [Doctoral Thesis, Maastricht University]. Maastricht University. https://doi.org/10.26481/dis.20210706bh

Document status and date:

Published: 01/01/2021

DOI:

10.26481/dis.20210706bh

Document Version:

Publisher's PDF, also known as Version of record

\section{Please check the document version of this publication:}

- A submitted manuscript is the version of the article upon submission and before peer-review. There can be important differences between the submitted version and the official published version of record.

People interested in the research are advised to contact the author for the final version of the publication, or visit the DOI to the publisher's website.

- The final author version and the galley proof are versions of the publication after peer review.

- The final published version features the final layout of the paper including the volume, issue and page numbers.

Link to publication

\footnotetext{
General rights rights.

- You may freely distribute the URL identifying the publication in the public portal. please follow below link for the End User Agreement:

www.umlib.nl/taverne-license

Take down policy

If you believe that this document breaches copyright please contact us at:

repository@maastrichtuniversity.nl

providing details and we will investigate your claim.
}

Copyright and moral rights for the publications made accessible in the public portal are retained by the authors and/or other copyright owners and it is a condition of accessing publications that users recognise and abide by the legal requirements associated with these

- Users may download and print one copy of any publication from the public portal for the purpose of private study or research.

- You may not further distribute the material or use it for any profit-making activity or commercial gain

If the publication is distributed under the terms of Article $25 \mathrm{fa}$ of the Dutch Copyright Act, indicated by the "Taverne" license above, 
Bregtje Catharina Maria Hermans

\section{Pulmonary Large Cell}

Neuroendocrine Carcinoma:

a unique type of lung cancer?
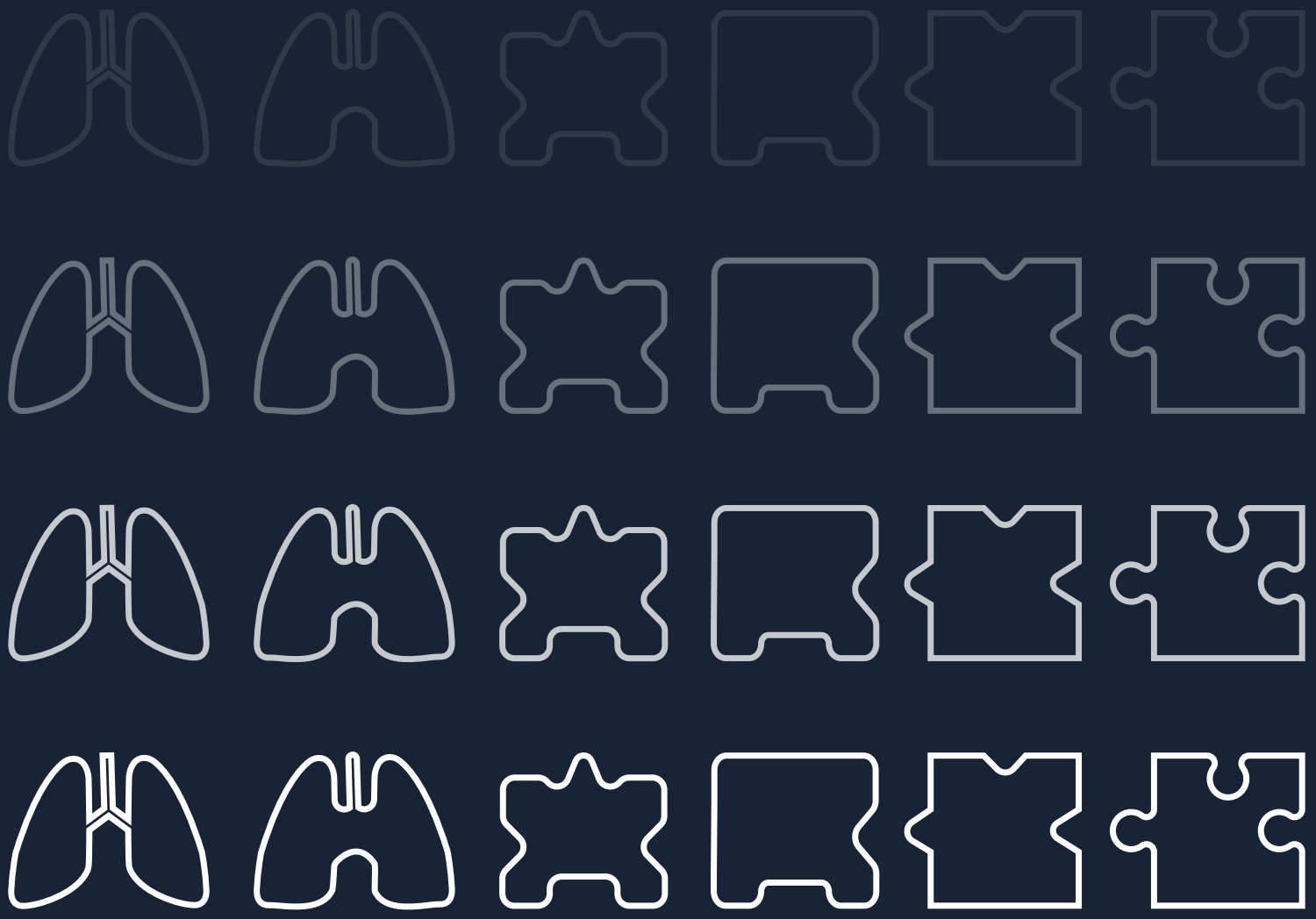

Identification of molecular and clinical subtypes $\varepsilon$ consequences for treatment 



\section{Pulmonary Large Cell Neuroendocrine Carcinoma: a unique type of lung cancer?}

Identification of molecular and clinical subtypes \& consequences for treatment 
(C) Bregtje Hermans, Maastricht 2021

For all articles published, the copyright has been transferred to the respective publisher. No part of this thesis may be reproduced in any form or by any means without written permission from the author or, when appropriate, from the publisher.

Cover: Sigrit Karsdorp

Lay-out: Tiny Wouters

Printed by: Ridderprint

ISBN: 978-94-6416-614-9

The research presented in this thesis was performed at the Department of Respiratory Medicine, GROW School for Oncology and Developmental Biology, Maastricht University Medical Centre+, Maastricht, the Netherlands.

Antibodies were provided by AbbVie for the study in chapter 7 of this thesis. The study in chapter 8 of this thesis was financially supported by Bristol-Myers Squibb.

Financial support for printing of this thesis was provided by Ipsen Farmaceutica B.V., Pfizer B.V. and Maastricht University. 


\title{
Pulmonary Large Cell Neuroendocrine Carcinoma: a unique type of lung cancer?
}

\section{Identification of molecular and clinical subtypes \& consequences for treatment}

\author{
PROEFSCHRIFT \\ ter verkrijging van de graad van doctor aan de Universiteit Maastricht, \\ op gezag van de Rector Magnificus, Prof.dr. Rianne M. Letschert \\ volgens het besluit van het College van Decanen, \\ in het openbaar te verdedigen \\ op dinsdag 6 juli 2021 om 16.00 uur
}

door

Bregtje Catharina Maria Hermans 


\section{Promotoren}

Prof. dr. A-M.C. Dingemans

Prof. dr. E-J.M. Speel

\section{Co-promotor}

Dr. J.L. Derks

\section{Beoordelingscommissie}

Prof. dr. A. Zur Hausen (voorzitter)

Prof. dr. F.H. Blackhall (University of Manchester / Christie NHS Foundation Trust, UK)

Prof. dr. V.C.G. Tjan-Heijnen

Prof. dr. M. Volante (University of Turin, Italy)

Prof. dr. M.A.G.G. Vooijs 


\section{Table of contents}

Chapter 1 General introduction and outline of this thesis $\quad 7$

Chapter 2 Exploring imaging features of molecular subtypes of large cell 21 neuroendocrine carcinoma (LCNEC)

Chapter 3 In-depth molecular analysis of combined and co-primary pulmonary large cell neuroendocrine carcinoma and adenocarcinoma

Chapter 4 Large cell neuroendocrine carcinoma with a solitary brain metastasis and low Ki-67: a unique subtype

Chapter 5 Pulmonary neuroendocrine neoplasms with well differentiated morphology and high proliferative activity: illustrated by a case series and review of the literature

Chapter 6 Delta like ligand 3 (DII3) as target for treatment of small cell lung 109 carcinoma (SCLC) and pulmonary large cell neuroendocrine carcinoma (LCNEC)

Chapter 7 DII3 expression in large cell neuroendocrine carcinoma (LCNEC) and 125 association with molecular subtypes and neuroendocrine profile

Chapter 8 Prevalence and prognostic value of $\mathrm{Pd}$-I1 expression in molecular subtypes of metastatic large cell neuroendocrine carcinoma (LCNEC)

Chapter 9 Unique metastatic patterns in neuroendocrine neoplasms of different primary origin

Chapter 10 General discussion

Addendum Summary

Samenvatting

Valorization

Dankwoord

List of publications

Curriculum Vitae 



\section{Chapter 1}

General introduction and outline of this thesis 



\section{General introduction and outline of this thesis}

Neuroendocrine neoplasms (NEN) can originate in different parts of the body, e.g. intestines, pancreas, prostate and lung. ${ }^{1}$ NEN is a rare disease with an incidence around 13 per 100.000 persons/year in the Netherlands. ${ }^{2}$ Pulmonary NENs are subdivided in well differentiated typical and atypical carcinoids (TC and AC) and poorly differentiated neuroendocrine carcinomas (NECs: small cell lung carcinoma (SCLC) and large cell neuroendocrine carcinoma (LCNEC)). ${ }^{1,3}$ Typical and atypical carcinoids form approximately $1-2 \%$ of all lung cancers. ${ }^{3,4}$ LCNEC is also rare, including approximately 1 $3 \%$ of new lung cancer cases, but incidence has increased over time. ${ }^{2,5-7}$ Although SCLC is the most prevalent form of a pulmonary NEN, it still contains only $\approx 15 \%$ of all pulmonary carcinoma. ${ }^{3,5}$ Nevertheless, SCLC and LCNEC are the two most prevalent forms of NEC throughout the body. ${ }^{2}$

\section{Pathological diagnosis of LCNEC}

LCNEC was first described in 1991 and was included in the World Health Organization (WHO)-classification in 1999. ${ }^{8}$ In 2015, LCNEC was removed from the non-small cell lung carcinoma (NSCLC) category and all pulmonary NEN were clustered into a single category of neuroendocrine lung tumors. ${ }^{3}$ Otherwise, the classification has not essentially changed. LCNEC is characterized by neuroendocrine morphology and nonsmall cells with a moderate to abundant amount of cytoplasm and presence of nucleoli. $^{3}$ This distinguishes LCNEC from SCLC, the latter having small cells, scant cytoplasm and a high nuclei to cytoplasmic ratio. ${ }^{3}$ Neuroendocrine morphology is identified by observation of rosette-like structure, formation of organoid nests, trabeculae and/or palisading of cells. Furthermore, a pepper and salt pattern is observed. $^{3}$ Besides neuroendocrine morphological differentiation, immunohistochemical expression of at least one neuroendocrine marker ( $>10 \%$ of the tumor; Synaptophysin, Chromogranin A or Neural Cell Adhesion Molecule 1 (Ncam1, Cd56) is required to confirm LCNEC diagnosis. ${ }^{3}$

LCNEC is distinguished from a carcinoid by evaluation of the mitotic index and necrosis (Table 1.1). Typical carcinoids have a mitotic index of $<2 / 2 \mathrm{~mm}^{2}$, atypical carcinoids of $2-10 / 2 \mathrm{~mm}^{2}$, whereas LCNEC and SCLC have a mitotic index $>10 / 2 \mathrm{~mm}^{2}{ }^{3}$ By definition, necrosis is not seen in TC, may be found in limited amounts in $A C$ and is often abundant in SCLC and LCNEC. ${ }^{3}$ Although most carcinoids present with well differentiated morphology (e.g. structured architecture and uniform and round or spindle shaped nuclei) and LCNEC mainly with poorly differentiated morphology (e.g. less structured, 
heterogeneous nuclei), morphological differentiation is not included as a criteria in the current WHO classification. Furthermore, Ki-67 proliferation index (PI) has been reported to be $<20 \%$ in the greater part of carcinoids and $>40 \%$ in most LCNEC and SCLC and might therefore support classification. ${ }^{9}$ Although Ki-67 seems to have prognostic relevance in pulmonary NEN, the additional prognostic value to the existing grading system has not been verified yet and $\mathrm{Ki}-67 \mathrm{PI}$ is not included in the current WHO classification. $^{3,9-14}$ After publication of the WHO classification in 2015, at least two exclusive molecular subtypes of LCNEC were identified by next generation sequencing. The first subtype harbors TP53 and RB1 mutations, resembling the most frequent mutations found in SCLC. The second subtype has TP53 and KEAP1/STK11 or KRAS mutations (NSCLC-like). ${ }^{15-17}$ Those two subtypes can also be distinguished by immunohistochemical pRb expression; the NSCLC-like LCNEC shows preserved nuclear immunostaining, whereas the SCLC-like LCNEC is characterized by loss of $p R b$ expression. $^{15}$

Table 1.1 Classification of pulmonary neuroendocrine neoplasms.

\begin{tabular}{lcccc}
\hline & Typical carcinoid & Atypical carcinoid & LCNEC & SCLC \\
\hline WHO criteria & & & & \\
Morphology & Non-small cell & Non-small cell & Non-small cell & Small cell \\
Mitotic index & $0-2 / 2 \mathrm{~mm}^{2}$ & $2-10 / 2 \mathrm{~mm}^{2}$ & $>10 / 2 \mathrm{~mm}^{2}$ & $>10 / 2 \mathrm{~mm}^{2}$ \\
Necrosis & Absent & Possible, focal & Frequent, abundant & Frequent, abundant \\
Non-WHO criteria & & & & \\
Differentiation & Well differentiated & Well differentiated & Poorly differentiated Poorly differentiated \\
Ki-67 PI & $\leq 20 \%$ & $\leq 20 \%$ & $>40 \%$ & $>40 \%$ \\
\hline
\end{tabular}

Abbreviations: LCNEC $=$ Large Cell Neuroendocrine Carcinoma, SCLC $=$ Small Cell Lung Carcinoma, WHO = World Health Organization, Ki-67 PI = Ki-67 proliferation index.

\section{Clinical characteristics}

LCNEC occurs most frequently in male patients with a median age of 65-70 years. ${ }^{5,6,18-21}$ The vast majority are smokers or former smokers. ${ }^{6,18,19,21}$ Presenting symptoms are comparable to NSCLC and SCLC: e.g. cough, hemoptysis, weight loss and/or fatigue. ${ }^{3,19}$ The carcinoid syndrome, which is sometimes present in carcinoids and is caused by hormonal production of somatostatin by tumor cells, is very rare in LCNEC. ${ }^{3,6,21}$ An X-ray or computed tomography (CT)-thorax is used during initial work-up of LCNEC patients, and commonly a peripheral tumor is observed.,22-27 An additional fluor-18deoxyglucose positron emission tomography (FDG-PET) scan is generally performed to evaluate dissemination of the tumor. ${ }^{19,26}$ In about half of the cases, patients present 
with metastatic disease at diagnosis. ${ }^{2,5,7,19,20}$ Median overall survival is 12-60 months for stage I-III patients, and only 4-9 months in patients with stage IV disease. ${ }^{5,7,20,21,28}$

\section{Treatment}

Due to the low incidence of LCNEC and a difficulty to diagnose LCNEC on a biopsy specimen, only few trials have evaluated optimal treatment strategies. Due to this lack of data, LCNEC is commonly treated using knowledge extrapolated from NSCLC and/or SCLC studies and their treatment guidelines. In case of localized disease, resection minimally by lobectomy is recommended..$^{29-31}$ In patients with stage II and III LCNEC, a multimodal approach including adjuvant chemotherapy (platinum + etoposide, or platinum + irinotecan) is advised. ${ }^{32-36}$ For stage I, the additional value of adjuvant chemotherapy is doubtful. However, recent retrospective series have shown a survival benefit in stage IB patients. ${ }^{30,31,37,38}$ For stage IA, conflicting results have been reported as some studies show a survival benefit of adjuvant chemotherapy but in others no differences in survival were found. ${ }^{30,37,38}$ Adjuvant radiotherapy seems not to be beneficial for LCNEC, although it might have a role in treatment of stage III. $^{29,30,37,39}$

Treatment of stage IV LCNEC is not discussed by the ESMO guideline of metastatic NSCLC. ${ }^{40}$ The ASCO guideline advises to treat stage IV LCNEC patients with palliative chemotherapy. ${ }^{41}$ Both a SCLC regimen (cisplatin/carboplatin + etoposide) or NSCLC regimen (platinum + gemcitabin/taxane) are deemed appropriate. ${ }^{41}$ Although some retrospective studies have shown an advantage for SCLC regimen, others did not report a significant difference. ${ }^{36,42,43}$ In the past years, retrospective series have investigated chemotherapeutic regimens in the two mutational subtypes of LCNEC (SCLC-like vs. NSCLC-like). In one study, improved survival was found in NSCLC-like LCNEC after treatment with NSCLC chemotherapy compared to SCLC regimen, whereas no difference was seen for SCLC-like LCNEC. ${ }^{15}$ However, in another study, no survival benefit of NSCLC regimen was observed in NSCLC-like LCNEC. ${ }^{44}$ Therefore, the most appropriate treatment in subtypes of LCNEC needs still to be validated.

Besides chemotherapy, some cases with durable responses ( $>6$ months) to immunotherapy have been reported. ${ }^{45-47}$ However, no data is available on immunotherapy in larger cohorts of LCNEC patients and data on Pd-I1 expression is scarce. ${ }^{46,48-54}$ Furthermore, some cases with targetable mutations or rearrangements known from NSCLC (e.g. epidermal growth factor receptor (EGFR), anaplastic lymphoma kinase $(A L K)$ ) with a durable responses (>6 months) to tyrosine kinase inhibitors (TKIS) have been reported. ${ }^{55-60}$ However, in some other cases no response 
was observed. ${ }^{56,61,62}$ Delta-like ligand 3 (DII3) has been identified as a potential therapeutic target in SCLC and LCNEC. Different approaches are used to develop drugs targeting DII3, but effectivity has not been validated so far. ${ }^{63-67}$ By now, no other targeted therapies for LCNEC have shown clinical benefit.

\section{Oncogenesis of LCNEC}

Oncogenesis of pulmonary NEN and more explicitly LCNEC has not been clarified yet. Overlapping characteristics of pulmonary neuroendocrine cells and NEN, e.g. expression of neuroendocrine markers, postulated neuroendocrine cells as cell of origin for NENs. ${ }^{68-70}$ However, neuroendocrine cells are scarce and it is disputed if all pulmonary NEN arise from those neuroendocrine cells, or that they may also originate from other pulmonary cell types. ${ }^{71}$ Most data on the cell of origin in pulmonary NEN is available for SCLC and as a matter of fact, it has been shown that inactivation of RB1 and TP53 in other pulmonary cells than neuroendocrine cells (e.g. basal cells and alveolar type II cells) can result in the development of SCLC. ${ }^{69,72,73}$ For LCNEC, limited data is available, but since SCLC and SCLC-like LCNEC have common mutational signatures, it is tempting to speculate that at least part of LCNEC have the same cell of origin as SCLC. However, development of different tumor types have been observed after deletion of RB1, PTEN and TP53 in various cell types of mice models. SCLC developed after targeting of basal cells only whereas targeting of general lung cells (e.g. alveolar cells, club-type cells) resulted in development of LCNEC in the majority of cases. ${ }^{73}$ Furthermore, SCLC is more often located centrally and LCNEC more often peripherally in the lung. ${ }^{23,74}$ Therefore, despite similar clinical and mutational characteristics, LCNEC might have different and/or additional cells of origin.

The most frequent mutations in LCNEC occur in the tumor suppressors TP53 (78-92\%) and $R B 1(38-47 \%){ }^{15-17}$ In SCLC, pRb is inactivated in the vast majority by $R B 1$ mutation (a.o. missense, nonsense, rearrangements and frameshifts). In RB1 wildtype tumors, other molecular alterations such as p16 inactivation, CDK5 upregulation and Acheatescute like 1 ( $A S C L 1$ ) overexpression can result in hyperphosphorylation and inactivation of intact $\mathrm{pRb}$ and these mechanisms might also be important in LCNEC. $^{75-77}$ STK11, KEAP1, Kirsten Rat Sarcoma (KRAS) and EGFR are frequently mutated in lung adenocarcinoma and can also be mutated in LCNEC (10-33\%, 18-31\%, 22\% and limited number of cases, respectively). ${ }^{15-17,58,62,78,79}$ KEAP1 and STK11 mutations contribute to oncogenesis by deregulation of metabolic processes. ${ }^{80,81}$ KRAS and EGFR mutations deregulate cell growth, differentiation and apoptosis. ${ }^{16,17,79}$ Additional proposed 
mechanisms underlying oncogenesis and especially neuroendocrine differentiation of LCNEC are alterations in the Notch-pathway, PI3K/AKT pathway, REST and SOX1. ${ }^{82-86}$ Loss of functional $\mathrm{pRb}$ might also have a role in neuroendocrine differentiation by BRN2 upregulation. $^{87}$ Therefore, it seems that LCNEC can originate from different pulmonary cells and that for oncogenesis an oncogenic driver (e.g. RB1 or KEAP1 mutation) in combination with an additional factor driving neuroendocrine differentiation (e.g. ASCL1 upregulation) is required (Figure 1.1).

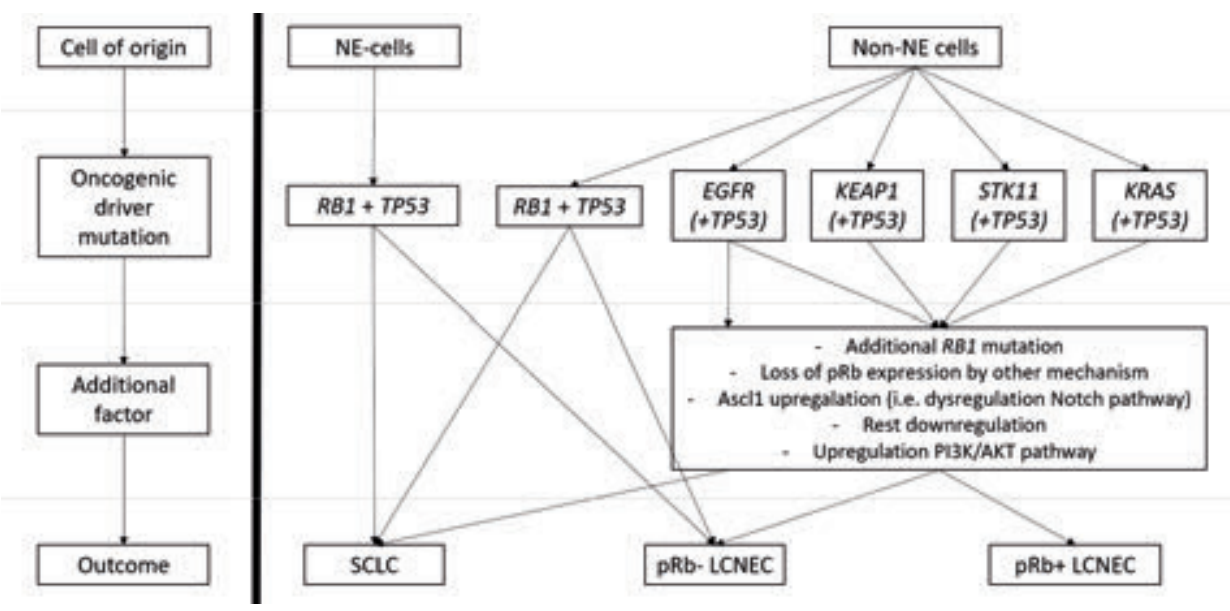

Figure 1.1 A simplified overview of possible ways for oncogenesis of SCLC and two molecular LCNEC subtypes. Abbreviations: $\mathrm{NE}$-cells = neuroendocrine cells, $\mathrm{SCLC}=$ small cell lung cancer, $\mathrm{pRb}$ LCNEC = large cell neuroendocrine carcinoma with loss of immunohistochemical $\mathrm{pRb}$ expression, $\mathrm{pRb}+\mathrm{LCNEC}=$ large cell neuroendocrine carcinoma with retained immunohistochemical $\mathrm{pRb}$ expression.

\section{Clinical subtypes of LCNEC}

According to the WHO classification, LCNEC is not further classified in clinical subtypes. However, some subtypes with particular clinical or pathological characteristics may exist. For example, LCNEC patients can present with only a solitary brain metastasis. This could be a subtype, because in case of stage IV disease, LCNEC generally presents as aggressive and disseminated disease. Another possible subtype constitutes of LCNEC patients with morphological or immunohistological signs of less aggressive behavior, e.g. well differentiated morphology and/or a relatively low Ki-67 PI. Identification of these patient groups might be relevant since prognosis and optimal treatment could be 
different from general LCNEC (i.e. more close to carcinoid), but clinical relevance has thus far not been established. Furthermore, some patients present with tumors having both an adenocarcinoma and LCNEC component or a co-primary tumor, one adenocarcinoma and one LCNEC. In cases where the LCNEC part and adenocarcinoma part of a tumor are clonally related, another cell than a neuroendocrine cell might be the cell of origin. A deeper understanding of those tumors could increase our knowledge on LCNEC oncogenesis.

\section{Aims and outline of this thesis}

The aim of this thesis is to obtain a deeper insight into relevant LCNEC molecular and clinical subtypes. Furthermore, predictive and prognostic markers will be evaluated within those subtypes, to indicate prognosis and guide optimal treatment strategies for individual LCNEC patients. Moreover, LCNEC is compared to NEN of other primary origins to reveal clinically relevant differences and similarities.

In chapter $\mathbf{2}$ of this thesis, a new method to differentiate between the molecular SCLClike and NSCLC-like LCNEC is investigated in a retrospective series of tumors, based on radiological features of $\mathrm{CT}$-scans at diagnosis.

In chapters 3-5 possible clinical subtypes of LCNEC are discussed. An in-depth analysis of combined tumors, consisting of both LCNEC and adenocarcinoma, is provided in chapter 3. Mutational and immunohistopathological characteristics of both tumor parts are compared to each other and to tumors with 'pure LCNEC' and 'pure adenocarcinoma'. In chapter $\mathbf{4}$ and $\mathbf{5}$ case series are described of selected LCNEC patients with a solitary brain metastasis or a well differentiated morphology, respectively. In both subtypes of LCNEC, prognostic immunohistological markers are proposed.

In chapter 6-8 two possible targeted therapies for LCNEC are discussed. An overview of current development and available evidence on DII3 targeted therapy in SCLC and LCNEC is provided in the review of chapter 6. DII3 expression in stage IV LCNEC and correlation with Ascl1 expression, expression of neuroendocrine markers and molecular subtypes is evaluated in chapter 7. Pd-I1 expression of LCNEC tumor cells is assessed in chapter 8. This expression is correlated to molecular subtypes and to expression of $\mathrm{Cd} 8$ positive cells within and outside the tumor and compared to $\mathrm{Pd}-\mathrm{I} 1$ expression in SCLC and NSCLC.

In chapter 9 overlap of LCNEC with other NEN is investigated. An overview of metastatic patterns at initial presentation is provided for NEN with gastro-intestinal, 
pancreatic, pulmonary, other and unknown primary origins. Both similarities and differences between the various primary organs are described.

In the general discussion in chapter 10, current evidence for subclassification of LCNEC and implications for WHO-classification are evaluated. Future possibilities for improved subclassification are proposed. Furthermore, possible predictive factors for systemic treatment are reviewed. Moreover, overlapping characteristics and differences between LCNEC and other NEN are discussed, with implications for treatment options and further research. 


\section{References}

1. Rindi G, Klimstra DS, Abedi-Ardekani B, et al. A common classification framework for neuroendocrine neoplasms: an International Agency for Research on Cancer (IARC) and World Health Organization (WHO) expert consensus proposal. Mod Pathol 2018;31:1770-1786.

2. Korse CM, Taal BG, van Velthuysen ML, et al. Incidence and survival of neuroendocrine tumours in the Netherlands according to histological grade: experience of two decades of cancer registry. Eur J Cancer 2013;49:1975-1983.

3. Travis WD, Brambilla E, Burke AP, et al. WHO Classification of Tumours of the Lung, Pleura, Thymus and Heart Lyon, France: International Agency for Research on Cancer; 2015.

4. Caplin ME, Baudin E, Ferolla $\mathrm{P}$, et al. Pulmonary neuroendocrine (carcinoid) tumors: European Neuroendocrine Tumor Society expert consensus and recommendations for best practice for typical and atypical pulmonary carcinoids. Ann Oncol 2015;26:1604-1620.

5. Derks JL, Hendriks LE, Buikhuisen WA, et al. Clinical features of large cell neuroendocrine carcinoma: a population-based overview. Eur Respir J 2016;47:615-624.

6. Takei $\mathrm{H}$, Asamura $\mathrm{H}$, Maeshima A, et al. Large cell neuroendocrine carcinoma of the lung: a clinicopathologic study of eighty-seven cases. J Thorac Cardiovasc Surg 2002;124:285-292.

7. Varlotto JM, Medford-Davis LN, Recht A, et al. Should large cell neuroendocrine lung carcinoma be classified and treated as a small cell lung cancer or with other large cell carcinomas? J Thorac Oncol 2011;6:1050-1058.

8. Travis WD, Linnoila RI, Tsokos MG, et al. Neuroendocrine tumors of the lung with proposed criteria for large-cell neuroendocrine carcinoma. An ultrastructural, immunohistochemical, and flow cytometric study of 35 cases. Am J Surg Pathol 1991;15:529-553.

9. Pelosi G, Rindi G, Travis WD, et al. Ki-67 antigen in lung neuroendocrine tumors: unraveling a role in clinical practice. J Thorac Oncol 2014;9:273-284.

10. Rindi G, Klersy C, Inzani F, et al. Grading the neuroendocrine tumors of the lung: an evidence-based proposal. Endocr Relat Cancer 2014;21:1-16.

11. Marchevsky AM, Hendifar A, Walts AE. The use of Ki-67 labeling index to grade pulmonary welldifferentiated neuroendocrine neoplasms: current best evidence. Mod Pathol 2018;31:1523-1531.

12. Swarts DR, Rudelius M, Claessen SM, et al. Limited additive value of the Ki-67 proliferative index on patient survival in World Health Organization-classified pulmonary carcinoids. Histopathology 2017;70:412-422.

13. Zahel T, Krysa S, Herpel E, et al. Phenotyping of pulmonary carcinoids and a Ki-67-based grading approach. Virchows Arch 2012;460:299-308.

14. Clay V, Papaxoinis G, Sanderson B, et al. Evaluation of diagnostic and prognostic significance of Ki-67 index in pulmonary carcinoid tumours. Clin Transl Oncol 2017;19:579-586.

15. Derks JL, Leblay N, Thunnissen E, et al. Molecular Subtypes of Pulmonary Large-cell Neuroendocrine Carcinoma Predict Chemotherapy Treatment Outcome. Clin Cancer Res 2018;24:33-42.

16. George J, Walter V, Peifer M, et al. Integrative genomic profiling of large-cell neuroendocrine carcinomas reveals distinct subtypes of high-grade neuroendocrine lung tumors. Nat Commun 2018;9:1048.

17. Rekhtman N, Pietanza MC, Hellmann MD, et al. Next-Generation Sequencing of Pulmonary Large Cell Neuroendocrine Carcinoma Reveals Small Cell Carcinoma-like and Non-Small Cell Carcinoma-like Subsets. Clin Cancer Res 2016;22:3618-3629.

18. Kinoshita T, Yoshida J, Ishii G, et al. The differences of biological behavior based on the clinicopathological data between resectable large-cell neuroendocrine carcinoma and small-cell lung carcinoma. Clin Lung Cancer 2013;14:535-540.

19. Grondahl V, Binderup T, Langer SW, et al. Characteristics of 252 patients with bronchopulmonary neuroendocrine tumours treated at the Copenhagen NET Centre of Excellence. Lung Cancer 2019;132:141-149.

20. Cao L, Li ZW, Wang M, et al. Clinicopathological characteristics, treatment and survival of pulmonary large cell neuroendocrine carcinoma: a SEER population-based study. PeerJ 2019;7:e6539. 
21. Asamura H, Kameya T, Matsuno $\mathrm{Y}$, et al. Neuroendocrine neoplasms of the lung: a prognostic spectrum. J Clin Oncol 2006;24:70-76.

22. Akata S, Okada S, Maeda J, et al. Computed tomographic findings of large cell neuroendocrine carcinoma of the lung. Clin Imaging 2007;31:379-384.

23. Zhou F, Hou L, Ding T, et al. Distinct clinicopathologic features, genomic characteristics and survival of central and peripheral pulmonary large cell neuroendocrine carcinoma: From different origin cells? Lung Cancer 2018;116:30-37.

24. Jung KJ, Lee KS, Han J, et al. Large cell neuroendocrine carcinoma of the lung: clinical, CT, and pathologic findings in 11 patients. J Thorac Imaging 2001;16:156-162.

25. Shin AR, Shin BK, Choi JA, et al. Large cell neuroendocrine carcinoma of the lung: radiologic and pathologic findings. J Comput Assist Tomogr 2000;24:567-573.

26. Lee KW, Lee $\mathrm{Y}$, Oh SW, et al. Large cell neuroendocrine carcinoma of the lung: CT and FDG PET findings. Eur J Radiol 2015;84:2332-2338.

27. Oshiro $\mathrm{Y}$, Kusumoto $\mathrm{M}$, Matsuno $\mathrm{Y}$, et al. CT findings of surgically resected large cell neuroendocrine carcinoma of the lung in 38 patients. AJR Am J Roentgenol 2004;182:87-91.

28. Raman V, Jawitz OK, Yang CJ, et al. Outcomes for Surgery in Large Cell Lung Neuroendocrine Cancer. J Thorac Oncol 2019;14:2143-2151.

29. Jiang $Y$, Lei $C$, Zhang $X$, et al. Double-edged role of radiotherapy in patients with pulmonary large-cell neuroendocrine carcinoma. J Cancer 2019;10:6422-6430.

30. Wakeam E, Adibfar A, Stokes S, et al. Defining the role of adjuvant therapy for early-stage large cell neuroendocrine carcinoma. J Thorac Cardiovasc Surg 2019.

31. Ogawa H, Tanaka Y, Kitamura Y, et al. Efficacy of perioperative chemotherapy for pulmonary high-grade neuroendocrine carcinomas: a propensity score matching analysis. J Thorac Dis 2019;11:1145-1154.

32. Tsoukalas N, Baxevanos P, Aravantinou-Fatorou E, et al. Advances on systemic treatment for lung neuroendocrine neoplasms. Ann Trans/ Med 2018;6:146.

33. Filosso PL, Guerrera F, Evangelista A, et al. Adjuvant chemotherapy for large-cell neuroendocrine lung carcinoma: results from the European Society for Thoracic Surgeons Lung Neuroendocrine Tumours Retrospective Database. Eur J Cardiothorac Surg 2017;52:339-345.

34. Iyoda A, Hiroshima K, Moriya Y, et al. Prospective study of adjuvant chemotherapy for pulmonary large cell neuroendocrine carcinoma. Ann Thorac Surg 2006;82:1802-1807.

35. Kenmotsu H, Niho S, Ito T, et al. A pilot study of adjuvant chemotherapy with irinotecan and cisplatin for completely resected high-grade pulmonary neuroendocrine carcinoma (large cell neuroendocrine carcinoma and small cell lung cancer). Lung Cancer 2014;84:254-258.

36. Rossi G, Cavazza A, Marchioni A, et al. Role of chemotherapy and the receptor tyrosine kinases KIT, PDGFRalpha, PDGFRbeta, and Met in large-cell neuroendocrine carcinoma of the lung. J Clin Oncol 2005;23:8774-8785.

37. Raman V, Jawitz OK, Yang CJ, et al. Adjuvant Therapy for Patients With Early Large Cell Lung Neuroendocrine Cancer: A National Analysis. Ann Thorac Surg 2019;108:377-383.

38. Kujtan L, Muthukumar V, Kennedy KF, et al. The Role of Systemic Therapy in the Management of Stage I Large Cell Neuroendocrine Carcinoma of the Lung. J Thorac Oncol 2018;13:707-714.

39. Prelaj A, Rebuzzi SE, Del Bene G, et al. Evaluation of the efficacy of cisplatin-etoposide and the role of thoracic radiotherapy and prophylactic cranial irradiation in LCNEC. ERJ open research 2017;3.

40. Planchard D, Popat S, Kerr K, et al. Metastatic non-small cell lung cancer: ESMO Clinical Practice Guidelines for diagnosis, treatment and follow-up. Ann Oncol 2018;29:iv192-iv237.

41. Hanna N, Johnson D, Temin S, et al. Systemic Therapy for Stage IV Non-Small-Cell Lung Cancer: American Society of Clinical Oncology Clinical Practice Guideline Update. J Clin Oncol 2017;35: 3484-3515.

42. Naidoo J, Santos-Zabala ML, Iyriboz T, et al. Large Cell Neuroendocrine Carcinoma of the Lung: ClinicoPathologic Features, Treatment, and Outcomes. Clin Lung Cancer 2016;17:e121-e129.

43. Sun JM, Ahn MJ, Ahn JS, et al. Chemotherapy for pulmonary large cell neuroendocrine carcinoma: similar to that for small cell lung cancer or non-small cell lung cancer? Lung Cancer 2012;77:365-370. 
44. Zhuo M, Guan Y, Yang X, et al. The prognostic and therapeutic role of genomic subtyping by sequencing tumor or cell-free DNA in pulmonary large-cell neuroendocrine carcinoma. Clin Cancer Res 2020; 26(4):892-901.

45. Levra MG, Mazieres J, C.A. V, et al. Efficacy of immune checkpoint inhibitors in large cell neuroendocrine lung cancer Journal of Thoracic Oncology 2017;12:S702-S703.

46. Wang VE, Urisman A, Albacker L, et al. Checkpoint inhibitor is active against large cell neuroendocrine carcinoma with high tumor mutation burden. J Immunother Cancer 2017;5:75.

47. Daido W, Yamasaki M, Saito N, et al. [Effectiveness of Nivolumab in Large-Cell Neuroendocrine Carcinoma of the Lung - A Report of Two Cases]. Gan To Kagaku Ryoho 2017;44:59-62.

48. Eichhorn F, Harms A, Warth A, et al. PD-L1 expression in large cell neuroendocrine carcinoma of the lung. Lung Cancer 2018;118:76-82.

49. Inamura K, Yokouchi Y, Kobayashi M, et al. Relationship of tumor PD-L1 (CD274) expression with lower mortality in lung high-grade neuroendocrine tumor. Cancer Med 2017;6:2347-2356.

50. Kasajima A, Ishikawa Y, Iwata A, et al. Inflammation and PD-L1 expression in pulmonary neuroendocrine tumors. Endocr Relat Cancer 2018;25:339-350.

51. Kim HS, Lee JH, Nam SJ, et al. Association of PD-L1 Expression with Tumor-Infiltrating Immune Cells and Mutation Burden in High-Grade Neuroendocrine Carcinoma of the Lung. J Thorac Oncol 2018;13: 636-648.

52. Tanaka $\mathrm{Y}$, Ogawa $\mathrm{H}$, Uchino $\mathrm{K}$, et al. Immunohistochemical studies of pulmonary large cell neuroendocrine carcinoma: a possible association between staining patterns with neuroendocrine markers and tumor response to chemotherapy. J Thorac Cardiovasc Surg 2013;145:839-846.

53. Tsuruoka K, Horinouchi H, Goto Y, et al. PD-L1 expression in neuroendocrine tumors of the lung. Lung Cancer 2017;108:115-120.

54. Hermans BCM, Derks JL, Thunnissen E, et al. Prevalence and prognostic value of PD-L1 expression in molecular subtypes of metastatic large cell neuroendocrine carcinoma (LCNEC). Lung Cancer 2019;130:179-186.

55. Shimizu N, Akashi Y, Fujii T, et al. Use of ALK Immunohistochemistry for Optimal Therapeutic Strategy of Pulmonary Large-cell Neuroendocrine Carcinoma and Identification of a Novel KIF5B-ALK Fusion Oncokinase. Anticancer Res 2019;39:413-420.

56. Zheng $Q$, Zheng $M$, Jin $Y$, et al. ALK-rearrangement neuroendocrine carcinoma of the lung: a comprehensive study of a rare case series and review of literature. Onco Targets Ther 2018;11: 4991-4998.

57. Wang $\mathrm{Y}$, Shen $\mathrm{YH}, \mathrm{Ma} \mathrm{S}$, et al. A marked response to icotinib in a patient with large cell neuroendocrine carcinoma harboring an EGFR mutation: A case report. Oncol Lett 2015;10:1575-1578.

58. De Pas TM, Giovannini M, Manzotti M, et al. Large-cell neuroendocrine carcinoma of the lung harboring EGFR mutation and responding to gefitinib. J Clin Oncol 2011;29:e819-822.

59. Ito M, Miyata $\mathrm{Y}$, Hirano S, et al. Therapeutic strategies and genetic profile comparisons in small cell carcinoma and large cell neuroendocrine carcinoma of the lung using next-generation sequencing. Oncotarget 2017;8:108936-108945.

60. Hayashi N, Fujita A, Saikai T, et al. Large Cell Neuroendocrine Carcinoma Harboring an Anaplastic Lymphoma Kinase (ALK) Rearrangement with Response to Alectinib. Internal medicine (Tokyo, Japan) 2018;57:713-716.

61. Le X, Desai NV, Majid A, et al. De novo pulmonary small cell carcinomas and large cell neuroendocrine carcinomas harboring EGFR mutations: Lack of response to EGFR inhibitors. Lung Cancer 2015;88:70-73.

62. Aroldi F, Bertocchi P, Meriggi F, et al. Tyrosine Kinase Inhibitors in EGFR-Mutated Large-Cell Neuroendocrine Carcinoma of the Lung? A Case Report. Case Rep Oncol 2014;7:478-483.

63. Saunders LR, Bankovich AJ, Anderson WC, et al. A DLL3-targeted antibody-drug conjugate eradicates high-grade pulmonary neuroendocrine tumor-initiating cells in vivo. Sci Transl Med 2015;7:302ra136.

64. Rudin CM, Pietanza MC, Bauer TM, et al. Rovalpituzumab tesirine, a DLL3-targeted antibody-drug conjugate, in recurrent small-cell lung cancer: a first-in-human, first-in-class, open-label, phase 1 study. Lancet Oncol 2017;18:42-51. 
65. Morgensztern D, Besse B, Greillier L, et al. Efficacy and Safety of Rovalpituzumab Tesirine in Third-Line and Beyond Patients with DLL3-Expressing, Relapsed/Refractory Small-Cell Lung Cancer: Results From the Phase II TRINITY Study. Clin Cancer Res 2019.

66. Giffin M, Cooke K, Lobenhofer E, et al. Targeting DLL3 with AMG 757, a BiTE ${ }^{\circledR}$ Antibody Construct, and AMG 119, a CAR-T, for the Treatment of SCLC. J Thorac Oncol 2018;13:Supplement.

67. Hipp S, Voynov V, Drobits-Handl B, et al. A novel T-cell engaging bispecific antibody induces specific and efficacious lysis of small cell lung cancer cells in vitro and potent $\mathrm{T}$ cell re-directed anti-tumor activity in vivo (Abstract \#549). AACR. Atlanta: 2019;Part A: Abstracts 1-2748.

68. Park KS, Liang MC, Raiser DM, et al. Characterization of the cell of origin for small cell lung cancer. Cell Cycle 2011;10:2806-2815.

69. Sutherland KD, Proost N, Brouns I, et al. Cell of origin of small cell lung cancer: inactivation of Trp53 and $\mathrm{Rb} 1$ in distinct cell types of adult mouse lung. Cancer Cell 2011;19:754-764.

70. Song $\mathrm{H}, \mathrm{Yao} \mathrm{E}$, Lin $\mathrm{C}$, et al. Functional characterization of pulmonary neuroendocrine cells in lung development, injury, and tumorigenesis. Proc Natl Acad Sci U S A 2012;109:17531-17536.

71. Gazdar AF, Savage TK, Johnson JE, et al. The comparative pathology of genetically engineered mouse models for neuroendocrine carcinomas of the lung. J Thorac Oncol 2015;10:553-564.

72. Yang D, Denny SK, Greenside PG, et al. Intertumoral Heterogeneity in SCLC Is Influenced by the Cell Type of Origin. Cancer Discov 2018;8:1316-1331.

73. Lazaro S, Perez-Crespo M, Lorz C, et al. Differential development of large-cell neuroendocrine or smallcell lung carcinoma upon inactivation of 4 tumor suppressor genes. Proc Natl Acad Sci U S A 2019;116:22300-22306.

74. Lee D, Rho JY, Kang S, et al. CT findings of small cell lung carcinoma: Can recognizable features be found? Medicine (Baltimore) 2016;95:e5426.

75. Gouyer V, Gazzeri S, Bolon I, et al. Mechanism of retinoblastoma gene inactivation in the spectrum of neuroendocrine lung tumors. Am J Respir Cell Mol Biol 1998;18:188-196.

76. Beasley MB, Lantuejoul S, Abbondanzo S, et al. The P16/cyclin D1/Rb pathway in neuroendocrine tumors of the lung. Hum Pathol 2003;34:136-142.

77. Meder L, Konig K, Ozretic L, et al. NOTCH, ASCL1, p53 and RB alterations define an alternative pathway driving neuroendocrine and small cell lung carcinomas. Int J Cancer 2016;138:927-938.

78. Cancer Genome Atlas Research N. Comprehensive molecular profiling of lung adenocarcinoma. Nature 2014;511:543-550.

79. Chapman AM, Sun KY, Ruestow P, et al. Lung cancer mutation profile of EGFR, ALK, and KRAS: Metaanalysis and comparison of never and ever smokers. Lung Cancer 2016;102:122-134.

80. Best SA, Sutherland KD. "Keaping" a lid on lung cancer: the Keap1-Nrf2 pathway. Cell Cycle 2018;17:1696-1707.

81. Gill RK, Yang SH, Meerzaman D, et al. Frequent homozygous deletion of the LKB1/STK11 gene in nonsmall cell lung cancer. Oncogene 2011;30:3784-3791.

82. D'Souza B, Miyamoto A, Weinmaster G. The many facets of Notch ligands. Oncogene 2008;27: 5148-5167.

83. Kunnimalaiyaan $\mathrm{M}$, Chen $\mathrm{H}$. Tumor suppressor role of Notch-1 signaling in neuroendocrine tumors. Oncologist 2007;12:535-542.

84. Chen C, Breslin MB, Lan MS. Sonic hedgehog signaling pathway promotes INSM1 transcription factor in neuroendocrine lung cancer. Cell Signal 2018;46:83-91.

85. Kreisler A, Strissel PL, Strick R, et al. Regulation of the NRSF/REST gene by methylation and CREB affects the cellular phenotype of small-cell lung cancer. Oncogene 2010;29:5828-5838.

86. Kan L, Israsena N, Zhang Z, et al. Sox1 acts through multiple independent pathways to promote neurogenesis. Dev Biol 2004;269:580-594.

87. Cobrinik D, Francis $\mathrm{RO}$, Abramson $\mathrm{DH}$, et al. $\mathrm{Rb}$ induces a proliferative arrest and curtails Brn-2 expression in retinoblastoma cells. Mol Cancer 2006;5:72. 



\section{Chapter 2}

\section{Exploring imaging features of molecular subtypes of large cell neuroendocrine carcinoma (LCNEC)}

B.C.M. Hermans, S. Sanduleanu, J.L. Derks, H. Woodruff, L. Hillen, R. Casale, F. Mohamed Hoesein, E. de Jong, D.M.H.J. ten Berge, E.J.M. Speel, P. Lambin, H.A. Gietema*, A-M.C. Dingemans*

* Contributed equally to this work Lung Cancer 2020;148:94-99 


\section{Abstract}

\section{Background}

Radiological characteristics and radiomics signatures can aid in differentiation between small cell lung carcinoma (SCLC) and non-small cell lung carcinoma (NSCLC). We investigated whether molecular subtypes of large cell neuroendocrine carcinoma (LCNEC), i.e. SCLC-like (with pRb loss) vs. NSCLC-like (with pRb expression), can be distinguished by imaging based on (1) imaging interpretation, (2) semantic features, and/or (3) a radiomics signature, designed to differentiate between SCLC and NSCLC.

\section{Methods}

Pulmonary oncologists and chest radiologists assessed chest CT-scans of 44 LCNEC patients for 'small cell-like' or 'non-small cell-like' appearance. The radiologists also scored semantic features of 50 LCNEC scans. Finally, a radiomics signature was trained on a dataset containing 48 SCLC and 76 NSCLC scans and validated on an external set of 58 SCLC and 40 NSCLC scans. This signature was applied on scans of 28 SCLC-like and 8 NSCLC-like LCNEC patients.

\section{Results}

Pulmonary oncologists and radiologists were unable to differentiate between molecular subtypes of LCNEC and no significant differences in semantic features were found. The area under the receiver operating characteristics curve of the radiomics signature in the validation set (SCLC vs. NSCLC) was 0.84 (95\% confidence interval (CI) 0.77-0.92) and 0.58 (95\% Cl 0.29-0.86) in the LCNEC dataset (SCLC-like vs. NSCLC-like).

\section{Conclusion}

LCNEC appears to have radiological characteristics of both SCLC and NSCLC, irrespective of pRb loss, compatible with the SCLC-like subtype. Imaging interpretation, semantic features and our radiomics signature designed to differentiate between SCLC and NSCLC were unable to separate molecular LCNEC subtypes, which underscores that LCNEC is a unique disease. 


\section{Introduction}

Large cell neuroendocrine carcinoma (LCNEC) of the lung is a rare tumor type, representing $1-3 \%$ of all types of lung cancer. ${ }^{1,2}$ The histological diagnosis of LCNEC is complex, and preferably, surgical resected tumor tissue is used. ${ }^{3}$ LCNEC can be separated in two main molecular subtypes: the first is SCLC-like (pathological SCLC-like, pSCLC-like), with co-mutation of RB1 and TP53 and loss of immunohistochemical (IHC) $\mathrm{pRb}$ expression and the second is NSCLC-like (pNSCLC-like), with co-mutation of TP53 and STK11/KEAP1/KRAS genes and preserved pRb expression. ${ }^{4-6}$ These subtypes might be predictive for chemotherapeutic responses. ${ }^{6,7}$

Over the past years efforts have been made to differentiate between the two main lung cancer subtypes, small cell lung carcinoma (SCLC) and non-small cell lung carcinoma (NSCLC), based on semantic features and radiomics signatures of routinely performed diagnostic chest CT-scans. ${ }^{8,9}$ Classic SCLC is described most commonly as a bulky central mass with major mediastinal lymph node involvement, whereas NSCLC is often located peripherally in the lungs with less extensive nodal involvement. ${ }^{3}$ In small case series almost exclusively consisting of stage I-III LCNEC, $0-36 \%$ of the tumors were located centrally. ${ }^{10-15}$

In this study we performed an in-depth analysis of CT-scans obtained in daily clinical practice to answer the following questions: 1) Are pulmonary oncologists and chest radiologists able to identify pSCLC-like and pNSCLC-like LCNEC based on their interpretation of radiological images? 2) Are there semantic features associated with molecular LCNEC subtypes and do the LCNEC subtypes resemble SCLC and NSCLC? Radiomics combines quantitative imaging features that can be extracted from standard-of-care medical imaging into so-called signatures. ${ }^{16,17}$ Therefore, we finally investigated 3) whether we could classify SCLC and NSCLC based on a radiomics signature and if we could use this signature to identify pSCLC-like and pNSCLC-like LCNEC, under the hypothesis that PSCLC-like LCNEC has comparable radiological characteristics as SCLC and pNSCLC-like LCNEC as NSCLC. 


\section{Materials and methods}

\section{Patient selection}

Diagnostic pretreatment CT-scans were requested for 158 patients with a confirmed LCNEC diagnosis after pathological review (Supplemental Figure S2.A). ${ }^{6} \mathrm{IHC}$ pRb (13A10) staining was performed on available formalin fixed paraffin embedded tissue, as described previously. ${ }^{6}$ Baseline CT-scans of 127 stage IV SCLC patients and 138 stage IV NSCLC patients were added to the study population (Supplemental Figure S2.B). ${ }^{18}$

The study protocol was approved by the medical ethical committee of the Maastricht UMC+ (METC azM/UM 14-4-043) and patient informed consent was waived due to the retrospective and anonymous nature of the study.

\section{Imaging interpretation}

A digital survey was developed with representative 2D images of CT-scans of LCNEC patients, from whom IHC pRb status was available $(\mathrm{N}=44)$ to evaluate the imaging interpretation by pulmonary oncologists (Qualtrics XM) (Supplemental Figure S2.C). Ten CT-scans of both SCLC and NSCLC patients were randomly included as controls (Supplemental Figure S2.A). The survey was distributed among all Dutch pulmonary oncologists, but only answers of clinicians with at least five years' experience were included in the analysis. Participants were asked to score for each CT-scan whether their first impression would be 'small cell', 'non-small cell' or 'not determinable based on the radiological image' and a 'combination score' was constructed for each scan (Supplemental methods S2.A and S2.B). Positive predictive values (PPVs) were calculated for imaging SCLC-like (iSCLC-like) and imaging NSCLC-like (iNSCLC-like) survey outcomes and association of molecular subtypes with survey outcome was investigated using the Fisher's exact test. A p-value $<0.05$ was considered significant.

\section{Semantic features}

To evaluate semantic features, next to the CT-scans used in the survey, additional scans of LCNEC patients with unknown IHC pRb status were included. Patients with missing slices of their CT-scan and those without a clear intrathoracic tumor were excluded (Supplemental Figure S2.A). Three experienced and dedicated chest radiologists (RC, $F M H, H G$ ) read the scans for semantic features (LCNEC N=50, SCLC N=10, NSCLC N=10) and a 'combination score' was constructed for each feature (Supplemental Methods S2.B, Supplemental Figure S2.D). Association of semantic features with pathological diagnosis was tested with the Fisher's exact test for cases with known IHC pRb status 
(LCNEC $\mathrm{N}=38$ ). Furthermore, the radiologists were asked to interpret the CT-scans in analogy to the pulmonary oncologists.

\section{Radiomics signature}

For evaluation of quantitative imaging features, additional scans from SCLC and NSCLC patients were added to the LCNEC and control scans used in the previous parts of this study (Supplemental Figure S2.B). The primary gross tumor volume of all scans was delineated by two investigators ( $\mathrm{SS}$ and $\mathrm{BH}$ ), supervised and checked by HG. Nondiagnostic CT-scans, including non-contrast enhanced CT-scans and scans without a well delimited intrapulmonary primary tumor were excluded. Furthermore, some scans had to be excluded due to technical problems with feature extraction (mostly variable slice spacing). The dataset was divided in a training set (SCLC ( $N=48)$ and $\operatorname{NSCLC~}(N=76))$ and external validation set (validation set $1, \operatorname{SCLC}(N=58)$ and $\operatorname{NSCLC~}(N=40)$ ). The resulting signature was applied to the dataset of LCNEC cases (validation set 2, pSCLClike $(\mathrm{N}=28)$ and pNSCLC-like ( $\mathrm{N}=8$ ) LCNEC) (Supplemental Figure S2.B). CT-image preprocessing, radiomics feature extraction, and feature harmonization are described extensively in Supplemental Methods S2.C.

\section{Results}

\section{Imaging interpretation}

The survey results of 23 pulmonary oncologists were used for analysis (Figure 2.1A). In the control group, the 2 patients by consensus allocated as iSCLC-like, were indeed SCLC (PPV 100\%), and 7/8 patients allocated as iNSCLC-like were NSCLC (PPV 88\%). In the LCNEC group, 1/44 was classified as iSCLC-like and 19/34 were classified as iNSCLClike. The only LCNEC allocated as iSCLC-like was also pSCLC-like (PPV 100\%). However, out of 19 patients regarded as iNSCLC-like, only 4 were pNSCLC-like, resulting in a PPV of $21 \%$ for an iNSCLC-like test being pNSCLC-like (Figure 2.1A, Supplemental Table S2.A).

The radiologists also scored 2 SCLCS as iSCLC-like (PPV 100\%), while only 6/9 iNSCLC-like scored cases represented NSCLC (PPV 66\%). In the LCNEC group, 2/38 were allocated to the iSCLC-like group and $8 / 38$ to the iNSCLC-like group. The PPV of an iSCLC-like scan to be a pSCLC-like LCNEC was $100 \%(2 / 2)$, but PPV of an iNSCLC-like scan to be pNSCLC-like LCNEC was only $13 \%(1 / 8)$ (Figure 2.1B, Supplemental Table S2.A). 

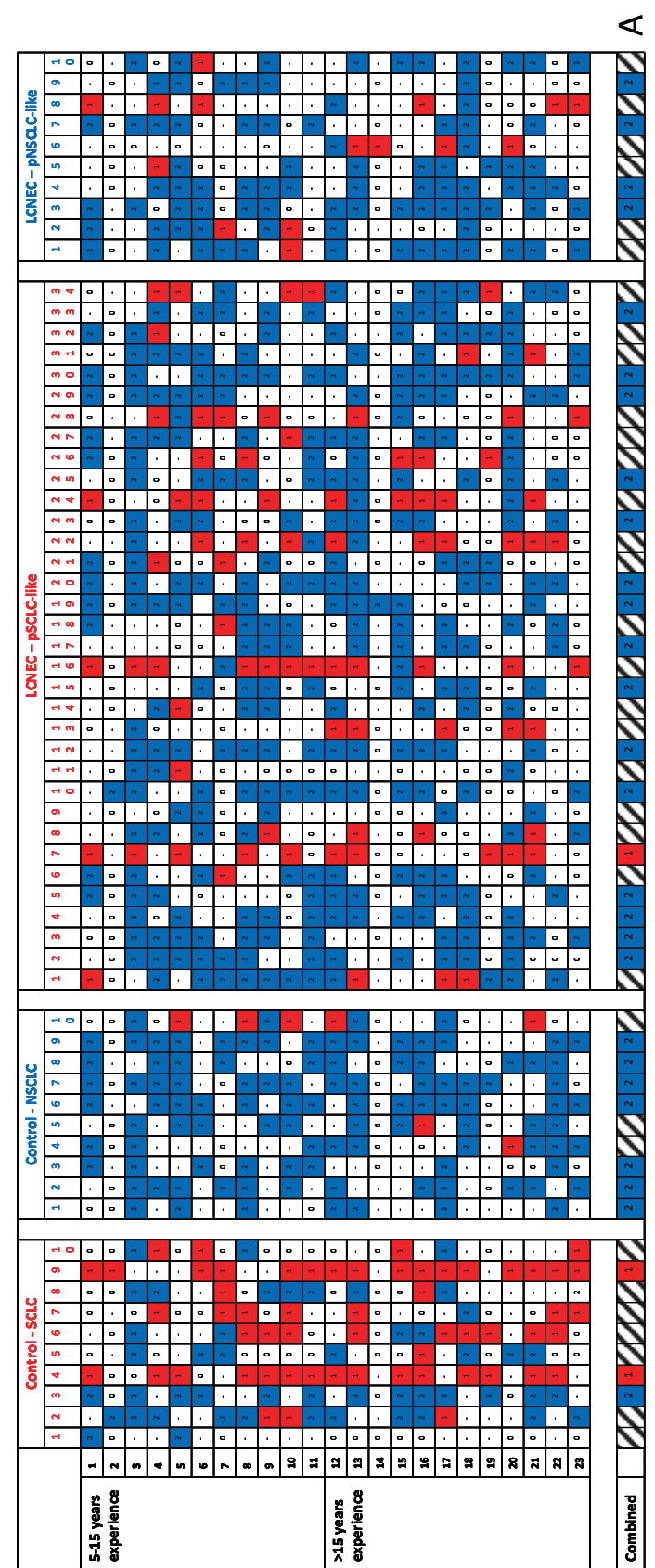

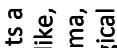

义

흥

है

논

들

刃

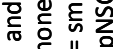

등 웜

它议

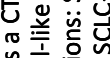

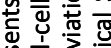

呕

흥 혼응응

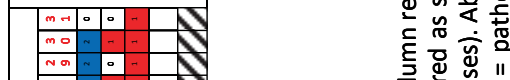

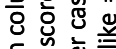

究 各至

商绕

نे

政

응

음 응 흥

Tㅇ

苛它足号

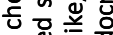

$m$ 这

등 웡

更斿

ธ。

苛立 品 $\overline{\bar{d}}$

은 令品

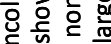

이음

충 든

苋蓄岸

든

홍

ฟ

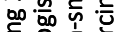

으응 흥

흥

入े

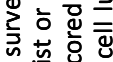
吅 능 융 可

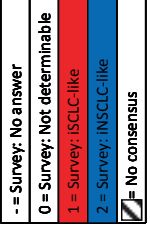

중

을 언

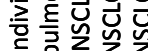

毛级

i

产 


\section{Semantic features}

An overview of all semantic features for 50 stage IV LCNEC is provided in Table 2.1. A peripheral location was more common than a central location (20/50 (40\%) vs. 9/50 $(18 \%))$, while in 21 cases location was not determinable/no consensus (42\%). In the control group, SCLC was more often located centrally compared to NSCLC (3/10 vs. $0 / 10, p=0.040$ ). No significant differences were observed in semantic features between 1) pSCLC-like and pNSCLC-like LCNEC and 2) other features of SCLC and NSCLC (Figure 2.2, Supplemental Table S2.B).

Table 2.1 Semantic features of CT-scans of patients with stage IV large cell neuroendocrine carcinoma $(\mathrm{N}=50)$.

\begin{tabular}{|c|c|}
\hline & LCNEC N (\%) \\
\hline Total number of patients & 50 \\
\hline \multicolumn{2}{|l|}{ Tumor location } \\
\hline Central & $9(18)$ \\
\hline Peripheral & $20(40)$ \\
\hline ND & $4(8)$ \\
\hline NC & $17(34)$ \\
\hline \multicolumn{2}{|l|}{ Involved lung lobe } \\
\hline LLL & $4(8)$ \\
\hline LUL & $22(44)$ \\
\hline RLL & $5(10)$ \\
\hline RML & $0(0)$ \\
\hline RUL & $14(28)$ \\
\hline ND & $4(8)$ \\
\hline NC & $1(2)$ \\
\hline \multicolumn{2}{|l|}{ Tumor size } \\
\hline$<3 \mathrm{~cm}$ & $10(20)$ \\
\hline $3-7 \mathrm{~cm}$ & $20(40)$ \\
\hline$>7 \mathrm{~cm}$ & $13(26)$ \\
\hline ND & $6(12)$ \\
\hline NC & $1(2)$ \\
\hline \multicolumn{2}{|l|}{ T } \\
\hline TO & 7 (14) \\
\hline $\mathrm{T} 1$ & $6(12)$ \\
\hline $\mathrm{T} 2$ & $10(20)$ \\
\hline T3 & $19(38)$ \\
\hline T4 & $0(0)$ \\
\hline ND & $3(6)$ \\
\hline NC & $5(10)$ \\
\hline \multicolumn{2}{|l|}{$\mathbf{N}$} \\
\hline No & $4(8)$ \\
\hline N1 & $2(4)$ \\
\hline N2 & $17(34)$ \\
\hline N3 & $24(48)$ \\
\hline ND & $1(2)$ \\
\hline NC & $2(4)$ \\
\hline
\end{tabular}


Table $2.1 \quad$ (continued)

\begin{tabular}{|c|c|}
\hline & $\begin{array}{c}\text { LCNEC } \\
\text { N (\%) }\end{array}$ \\
\hline \multicolumn{2}{|l|}{ Liver metastases } \\
\hline No & $26(52)$ \\
\hline Limited & $5(10)$ \\
\hline Diffuse & $9(18)$ \\
\hline ND & $9(18)$ \\
\hline $\mathrm{NC}$ & $1(2)$ \\
\hline \multicolumn{2}{|l|}{ Aspect tumor } \\
\hline Homogeneous & $16(32)$ \\
\hline Heterogeneous & $29(58)$ \\
\hline ND & $2(4)$ \\
\hline NC & $3(6)$ \\
\hline \multicolumn{2}{|l|}{ Tumor border* } \\
\hline Smooth & $5(10)$ \\
\hline Lobulated & $23(46)$ \\
\hline Spiculated & $26(52)$ \\
\hline \multicolumn{2}{|l|}{ Internal characteristics* } \\
\hline Calcification & $6(12)$ \\
\hline Necrosis & $10(20)$ \\
\hline Air bronchogram & $10(20)$ \\
\hline Cavitation & $1(2)$ \\
\hline Pleural invasion & $15(30)$ \\
\hline Notching & $0(0)$ \\
\hline \multicolumn{2}{|c|}{ External characteristics* } \\
\hline Groundglass & $12(24)$ \\
\hline Bubble lucencies & $0(0)$ \\
\hline Open bronchus sign & $0(0)$ \\
\hline Pleural tag & $7(14)$ \\
\hline Distal mucus plug & $1(2)$ \\
\hline Distal atelectasis & $6(12)$ \\
\hline Pleural fluid & $3(6)$ \\
\hline Satellite lesions & $18(36)$ \\
\hline Emphysema & $24(48)$ \\
\hline
\end{tabular}

* Multiple answers possible for each scan. Abbreviations: $L C N E C=$ large cell neuroendocrine carcinoma, ND = Not determinable (could not be determined by $\geq 2 / 3$ radiologists), NC $=$ No consensus (no majority $(\geq 2 / 3$ ) for one answer ( $3 / 3$ for tumor location)), LLL = left lower lobe, $L U L=$ left upper lobe, RLL = right lower lobe, RML $=$ right middle lobe, $\mathrm{RUL}=$ right upper lobe.

\section{Radiomics signature}

A dataset of scans of SCLC and NSCLC patients was used to train a random forest model to separate both tumor types (Supplemental Figure S2.B, Supplemental Figure S2.E). The area under the operating characteristics curve (AUC) for this model was 0.84 (95\% confidence interval ( $\mathrm{Cl}$ ) 0.76-0.92) and for the external validation set of SCLC and NSCLC 0.84 (95\% Cl 0.77-0.92). The validated model was applied to the scans of pSCLC-like and pNSCLC-like LCNEC patients, which resulted in an AUC of 0.58 (95\% Cl $0.29-0.86$ ) 
(Supplemental Figures S2.F-H). According to the model 7/36 LCNEC were allocated to the SCLC category and 29/36 to the NSCLC category. In the subtypes, 4/28 scans of pSCLC-like LCNEC were allocated to the SCLC category and 5/8 pNSCLC-like LCNEC were allocated to the NSCLC category (Figure 2.2). The PPV of a SCLC category outcome of the model to be a pSCLC-like LCNEC was therefore $57 \%$. The PPV of a NSCLC category outcome of the model to be pNSCLC-like LCNEC was only $17 \%$.
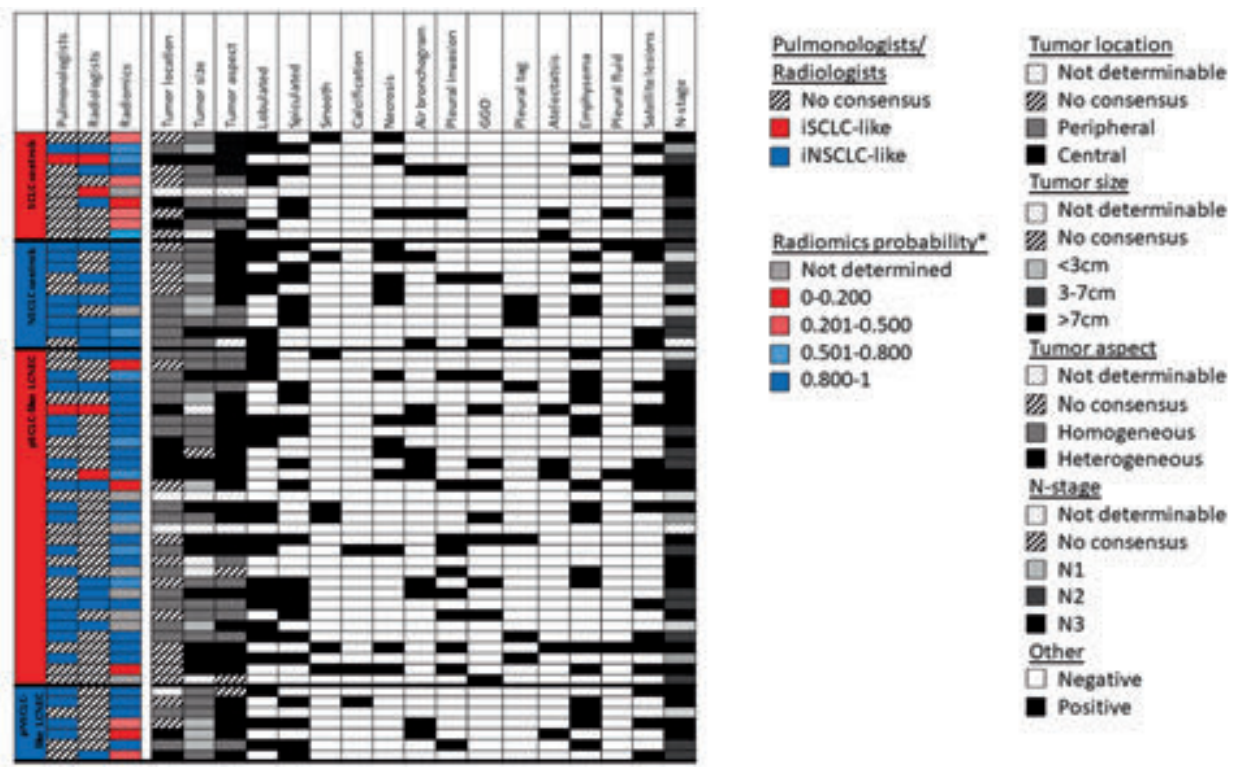

Figure 2.2 Clinical interpretation of CT-scans by pulmonary oncologists and radiologists, probability score of radiomics signature and semantic features of subtypes of stage IV large cell neuroendocrine carcinoma and control scans of small cell lung carcinoma and non-small cell lung carcinoma. *Radiomics probability: Close to 0 more likely to be SCLC, close to 1 more likely to be NSCLC. Abbreviations: $\mathrm{SCLC}=$ small cell lung carcinoma, NSCLC $=$ non-small cell lung carcinoma, LCNEC $=$ large cell neuroendocrine carcinoma, pSCLC-like = pathological SCLC-like, pNSCLC-like = pathological NSCLC-like, iSCLC-like = imaging SCLC-like, iNSCLC-like = imaging NSCLC-like, GGO = ground glass opacities, $\mathrm{N}$-stage $=$ nodal stage .

\section{Discussion}

In this study, we have investigated whether pSCLC-like and pNSCLC-like stage IV LCNEC could be distinguished on CT-scans based on imaging interpretation, semantic features or a radiomics signature. Pathological diagnosis of LCNEC is complicated and preferably, surgical resected tumor tissue is used. ${ }^{3}$ However, patients with LCNEC often present 
with disseminated disease and the diagnosis is generally based on small tumor biopsies, that are not always conclusive regarding the histological subtype of the tumor, requiring larger and/or repeated biopsies. ${ }^{1}$ Here, we tried to find less invasive alternatives to subclassify LCNEC. The radiomics signature trained on scans of SCLC and NSCLC patients was able to identify SCLC and NSCLC in an external validation set. However, pSCLC-like and pNSCLC-like LCNEC could not be separated by this signature. The subclassification between molecular LCNEC subtypes could neither be made based on imaging interpretation or semantic features. Moreover, LCNEC cases showed features of both SCLC and NSCLC, showing that LCNEC is a separate entity.

In this study, experienced pulmonary oncologists and chest radiologists could fairly differentiate between SCLC and NSCLC based features provided by CT-scans. However, no difference between pSCLC-like and pNSCLC-like LCNEC could be identified. So far, only one study of 8 LCNEC patients found 3/4 pSCLC-like LCNEC to be located central and $3 / 4$ pNSCLC-like LCNEC to be located peripheral. ${ }^{10}$ Based on the results of our study, in case the interpretation of a CT-scan of stage IV lung cancer by consensus is 'small cell-like', pathologic investigations will probably confirm SCLC morphology or pSCLC-like LCNEC. In contrast, if the interpretation is 'non-small cell-like', pathology can still reveal SCLC or pSCLC-like LCNEC, and no clinical consequences should be imposed.

Semantic features in a cohort of 50 stage IV LCNEC patients have not been investigated previously. The percentages of semantic features we found are in general comparable to those of smaller series including mainly stage I-III LCNEC (Table 2.2). ${ }^{11-15,19}$ Most semantic features in LCNEC were identified in percentages in between percentages previously described for SCLC and NSCLC. For example, the percentage of central LCNEC lesions was in between that of SCLC and NSCLC and similar patterns were seen for pleural tags, distal atelectases, liver metastases and N-stage. ${ }^{11,12,14,18}$ This indicates that LCNEC is a unique disease with characteristics of both SCLC and NSCLC.

We created an accurate radiomics signature that was able to classify SCLC and NSCLC based on CT-scans. To the best of our knowledge, only two studies, both without external validation, have reported on the separation of SCLC and NSCLC, constructing signatures with an AUC of 0.74 (95\% $\mathrm{Cl} 0.68-0.80$ ) and $>0.60$, respectively. ${ }^{8,9}$ Despite the good performance of our signature in the SCLC vs. NSCLC external validation set, our model was unable to separate pSCLC-like and pNSCLC-like LCNEC. This indicates that pSCLC-like LCNEC and SCLC as well as PNSCLC-like LCNEC and NSCLC have different quantitative imaging features. This further adds to the unique characteristics of LCNEC compared to both SCLC and NSCLC. 


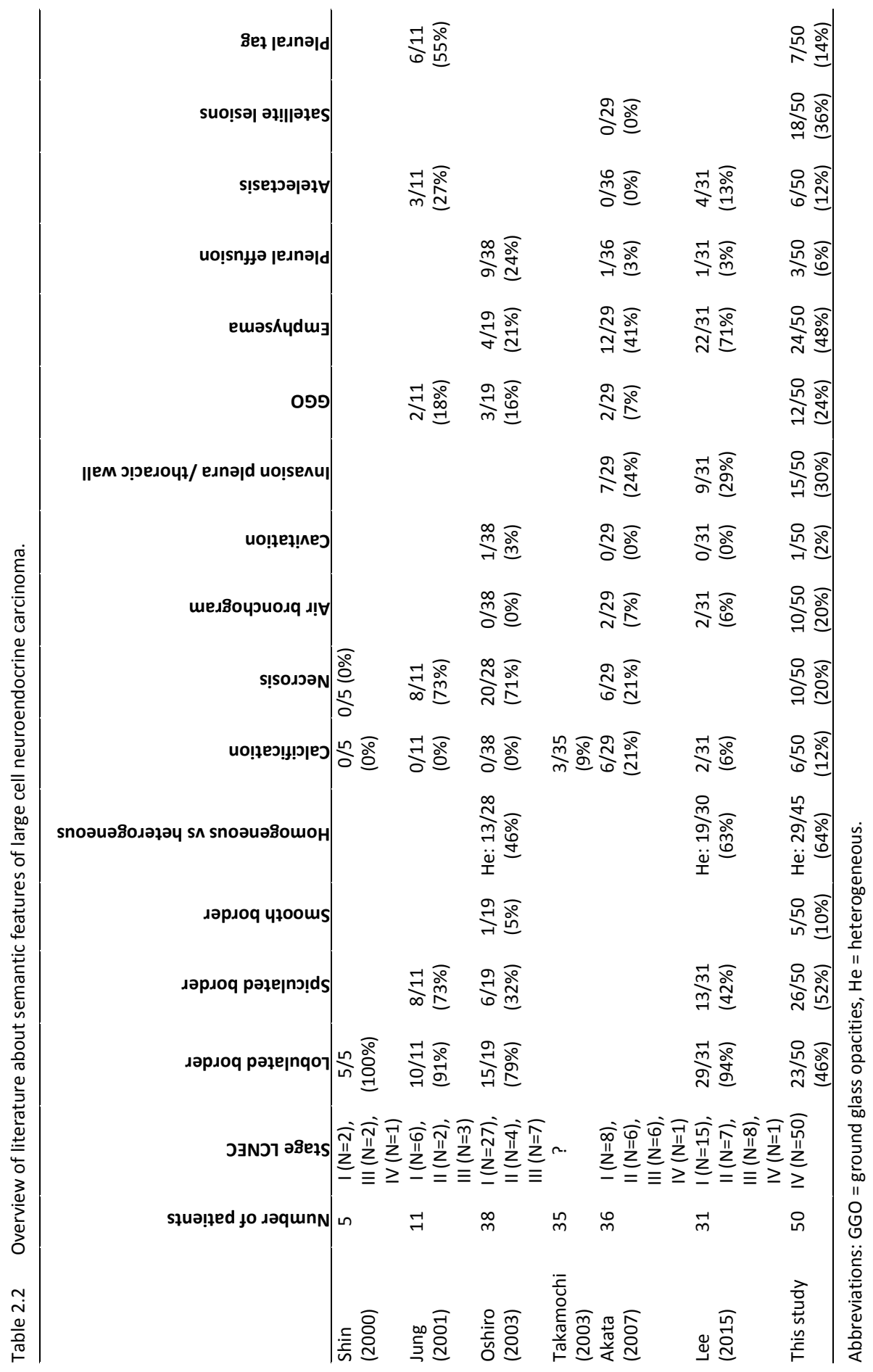


This study has several limitations. First, a limited number of CT-scans of stage IV LCNEC patients was available for this study due to the rarity of LCNEC and various technical and regulatory bottlenecks to obtain scans from multiple hospitals. Furthermore, development of the radiomics model was complicated by quite high heterogeneity in the applied scanning protocol, probably due to the long time frame in which the examinations were performed (2003-2018) and the large number of hospitals included from all over the Netherlands. To correct for inter-scanner model, acquisition protocol and reconstruction settings variation, we used the ComBat statistical harmonization technique available for multicenter imaging studies before developing the radiomics signature. ${ }^{20-22}$

In conclusion, LCNEC has radiological characteristics of both SCLC and NSCLC, but these characteristics do not correlate with pSCLC-like and pNSCLC-like LCNEC subtypes based on imaging interpretation by pulmonary oncologists and radiologists, semantic features or a radiomics signature designed to differentiate between SCLC and NSCLC. Most LCNEC were classified by clinicians and radiomics as NSCLC-like despite SCLC-like molecular characteristics, highlighting LCNEC as a unique tumor entity.

\section{Acknowledgement}

We thank E. van den Broek and colleagues from PALGA for selection of LCNEC patients from the PALGA database and providing pathological material. We thank all pulmonologists who provided CT-scans of LCNEC patients. We would also like to thank the pulmonary oncologists who participated in this study by filling out the survey on imaging interpretation. 


\section{References}

1. Derks JL, Hendriks LE, Buikhuisen WA, et al. Clinical features of large cell neuroendocrine carcinoma: a population-based overview. Eur Respir J 2016;47:615-624.

2. Takei $\mathrm{H}$, Asamura $\mathrm{H}$, Maeshima $\mathrm{A}$, et al. Large cell neuroendocrine carcinoma of the lung: a clinicopathologic study of eighty-seven cases. J Thorac Cardiovasc Surg 2002;124:285-292.

3. Travis WD, Brambilla E, Burke AP, et al. WHO Classification of Tumours of the Lung, Pleura, Thymus and Heart Lyon, France: International Agency for Research on Cancer; 2015.

4. Rekhtman N, Pietanza MC, Hellmann MD, et al. Next-Generation Sequencing of Pulmonary Large Cell Neuroendocrine Carcinoma Reveals Small Cell Carcinoma-like and Non-Small Cell Carcinoma-like Subsets. Clin Cancer Res 2016;22:3618-3629.

5. George J, Walter V, Peifer M, et al. Integrative genomic profiling of large-cell neuroendocrine carcinomas reveals distinct subtypes of high-grade neuroendocrine lung tumors. Nat Commun 2018;9:1048.

6. Derks JL, Leblay N, Thunnissen E, et al. Molecular Subtypes of Pulmonary Large-cell Neuroendocrine Carcinoma Predict Chemotherapy Treatment Outcome. Clin Cancer Res 2018;24:33-42.

7. Zhuo M, Guan Y, Yang X, et al. The Prognostic and Therapeutic Role of Genomic Subtyping by Sequencing Tumor or Cell-Free DNA in Pulmonary Large-Cell Neuroendocrine Carcinoma. Clin Cancer Res 2020;26:892-901.

8. E L, Lu L, Li L, et al. Radiomics for Classification of Lung Cancer Histological Subtypes Based on Nonenhanced Computed Tomography. Acad Radiol 2018.

9. E L, Lu L, Li L, et al. Radiomics for Classifying Histological Subtypes of Lung Cancer Based on Multiphasic Contrast-Enhanced Computed Tomography. J Comput Assist Tomogr 2019;43:300-306.

10. Zhou F, Hou L, Ding T, et al. Distinct clinicopathologic features, genomic characteristics and survival of central and peripheral pulmonary large cell neuroendocrine carcinoma: From different origin cells? Lung Cancer 2018;116:30-37.

11. Jung KJ, Lee KS, Han J, et al. Large cell neuroendocrine carcinoma of the lung: clinical, CT, and pathologic findings in 11 patients. J Thorac Imaging 2001;16:156-162.

12. Akata S, Okada S, Maeda J, et al. Computed tomographic findings of large cell neuroendocrine carcinoma of the lung. Clin Imaging 2007;31:379-384.

13. Shin AR, Shin BK, Choi JA, et al. Large cell neuroendocrine carcinoma of the lung: radiologic and pathologic findings. J Comput Assist Tomogr 2000;24:567-573.

14. Lee KW, Lee $\mathrm{Y}$, Oh SW, et al. Large cell neuroendocrine carcinoma of the lung: CT and FDG PET findings. Eur J Radiol 2015;84:2332-2338.

15. Oshiro $\mathrm{Y}$, Kusumoto $\mathrm{M}$, Matsuno $\mathrm{Y}$, et al. CT findings of surgically resected large cell neuroendocrine carcinoma of the lung in 38 patients. AJR Am J Roentgenol 2004;182:87-91.

16. Lambin P, Leijenaar RTH, Deist TM, et al. Radiomics: the bridge between medical imaging and personalized medicine. Nat Rev Clin Oncol 2017;14:749-762.

17. Lambin P, Rios-Velazquez E, Leijenaar R, et al. Radiomics: extracting more information from medical images using advanced feature analysis. Eur J Cancer 2012;48:441-446.

18. Rizzo S, Raimondi S, de Jong EEC, et al. Genomics of non-small cell lung cancer (NSCLC): Association between CT-based imaging features and EGFR and K-RAS mutations in 122 patients-An external validation. Eur J Radiol 2019;110:148-155.

19. Takamochi K, Yokose T, Yoshida J, et al. Calcification in large cell neuroendocrine carcinoma of the lung. Jpn J Clin Oncol 2003;33:10-13.

20. Johnson WE, Li C, Rabinovic A. Adjusting batch effects in microarray expression data using empirical Bayes methods. Biostatistics (Oxford, England) 2007;8:118-127.

21. Lucia F, Visvikis D, Vallieres M, et al. External validation of a combined PET and MRI radiomics model for prediction of recurrence in cervical cancer patients treated with chemoradiotherapy. European journal of nuclear medicine and molecular imaging 2019;46:864-877.

22. Orlhac F, Frouin F, Nioche C, et al. Validation of A Method to Compensate Multicenter Effects Affecting CT Radiomics. Radiology 2019;291:53-59. 


\section{Supplemental material}

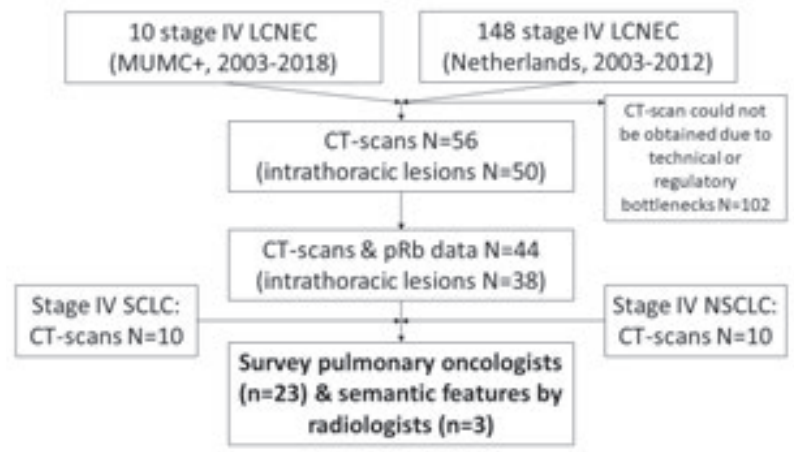

Figure S2.A Selection of CT-scans of LCNEC, SCLC and NSCLC patients for survey among pulmonary oncologists and for scoring of semantic features by radiologists. Abbreviations: LCNEC = large cell neuroendocrine carcinoma, MUMC $+=$ Maastricht University Medical Centre,$+ \mathrm{SCLC}=$ small cell lung carcinoma, NSCLC = non-small cell lung carcinoma.
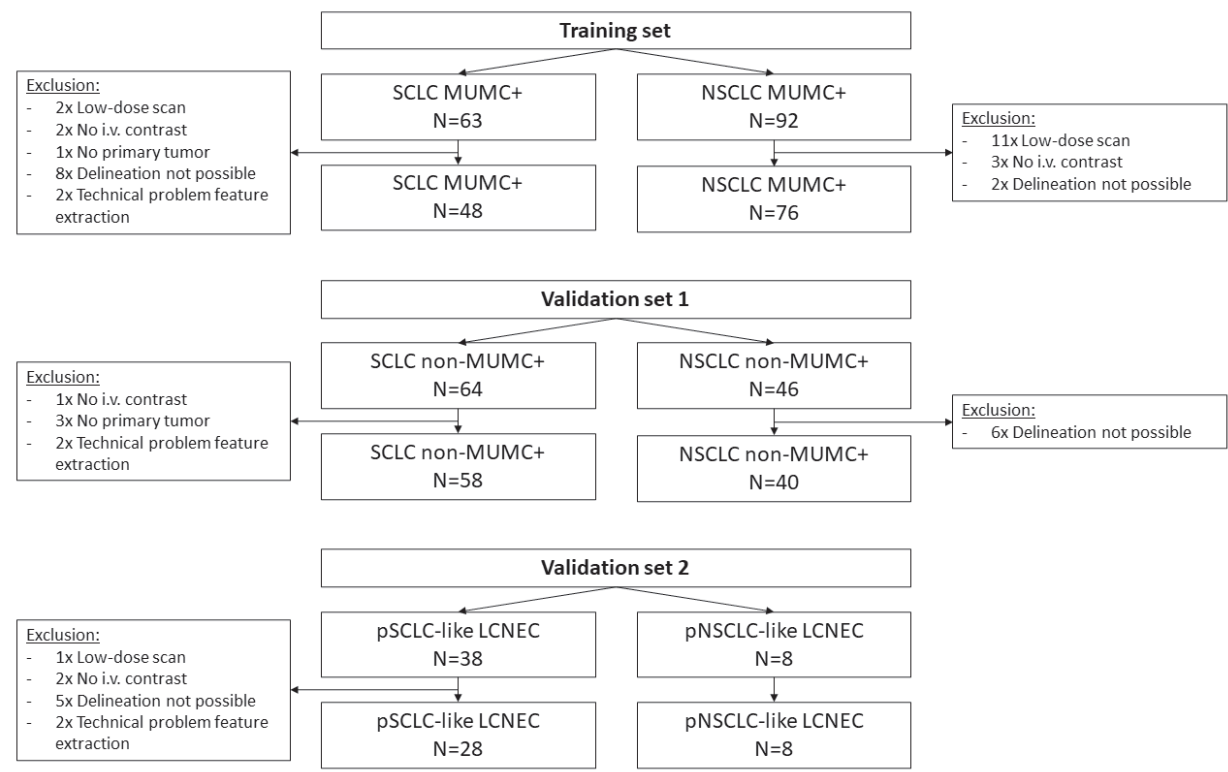

Figure S2.B Selection of CT-scans for training set of radiomics signature (SCLC and NSCLC from Maastricht University Medical Center+), validation set 1 (SCLC and NSCLC from Erasmus Medical Center and Zuyderland Hospital) and validation set 2 (pathological SCLC-like and NSCLC-like LCNEC, different centers in the Netherlands). Abbreviations: SCLC = small cell lung carcinoma, NSCLC = non-small cell lung carcinoma, MUMC+ = Maastricht University Medical Center+, i.v. = intravenous, PSCLC-like = pathological SCLC-like, LCNEC = large cell neuroendocrine carcinoma, pNSCLC-like $=$ pathological NSCLC-like. 

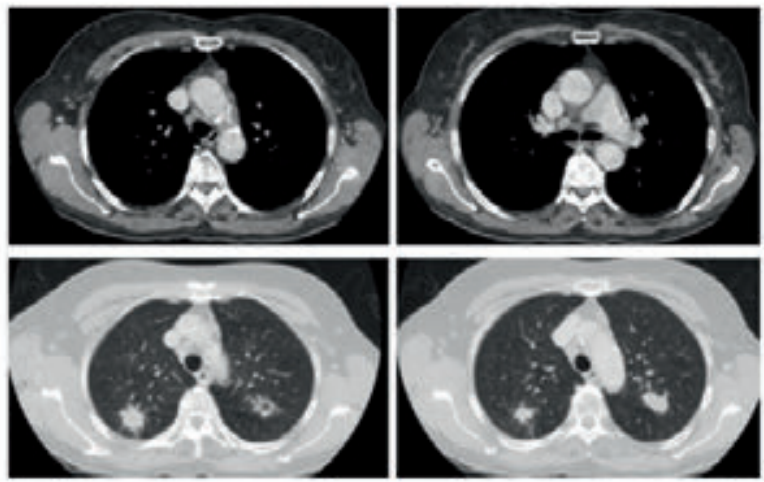

Small cell tung carcinoma like

Non-small cell lung carcinoma like

Not determinable based on the radiological image

Figure S2.C Survey among pulmonary oncologists to give an interpretation of CT-scans to be small cell-like or non-small cell-like. 


\section{Tumor location}
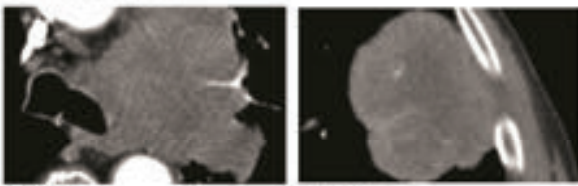

Central

Peripheral
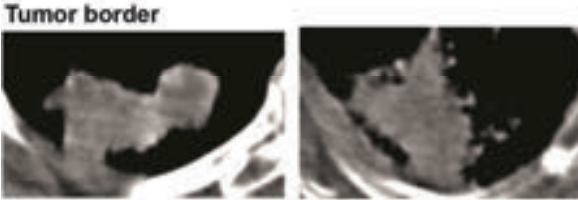

Lobulated

Spiculated

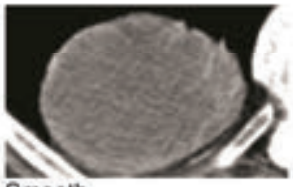

Smooth

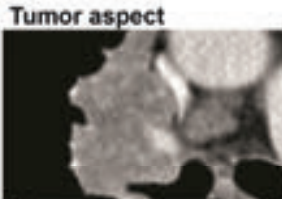

Heterogeneous

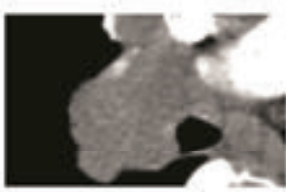

Homogeneous

\section{Internal characteristics}
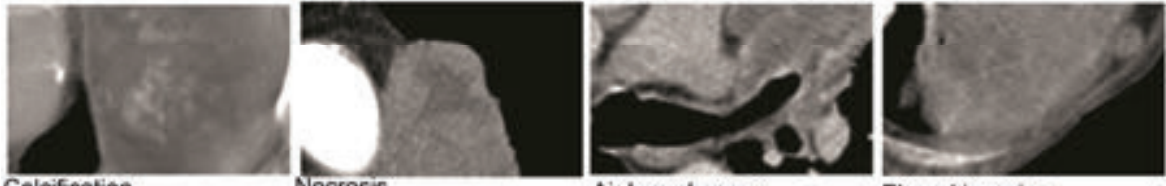

Calcification

Pleural invasion

\section{External characteristics}

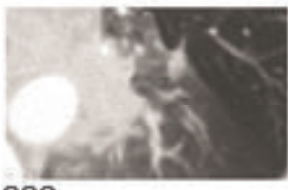

GGO

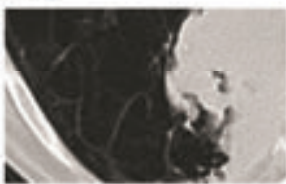

Emphysema

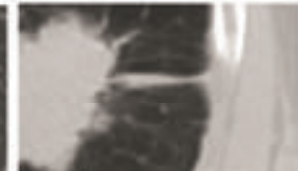

Pleural tag

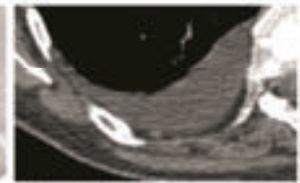

Pleural fluid

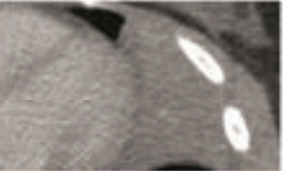

Atelectasis

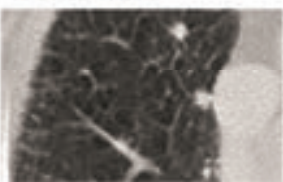

Satellite lesions

Figure S2.D Representative images of semantic features in stage IV small cell lung carcinoma, non-small cell lung carcinoma and large cell neuroendocrine carcinoma patients, as assessed by radiologists. Abbreviation: GGO = ground glass opacities. 


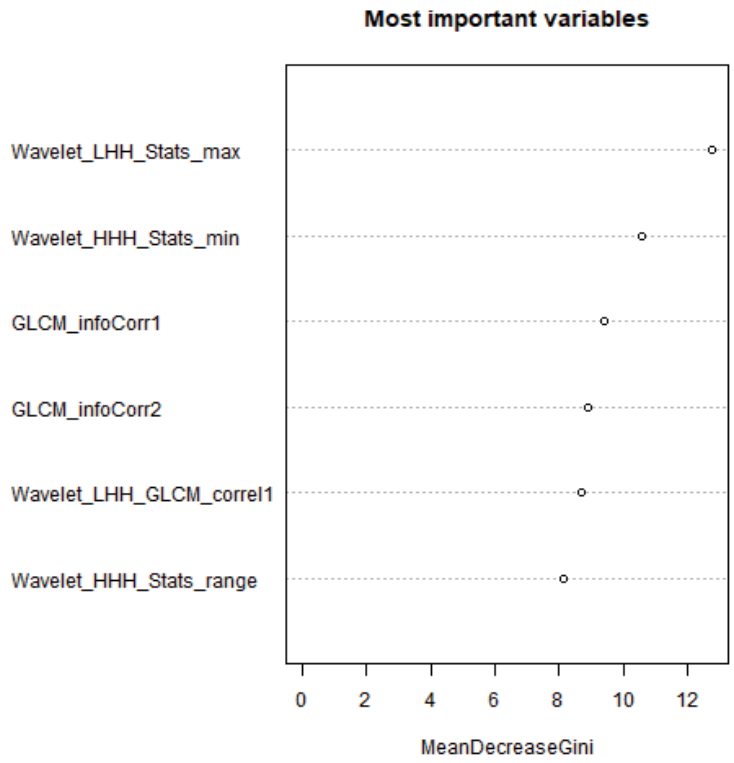

Figure S2.E Features in final model and their importance in the model. Mean Decrease in Gini is the average of a variable's total decrease in node impurity, weighted by the proportion of samples reaching that node in each individual decision tree in the random forest. A higher Mean Decrease in Gini indicates higher variable importance.

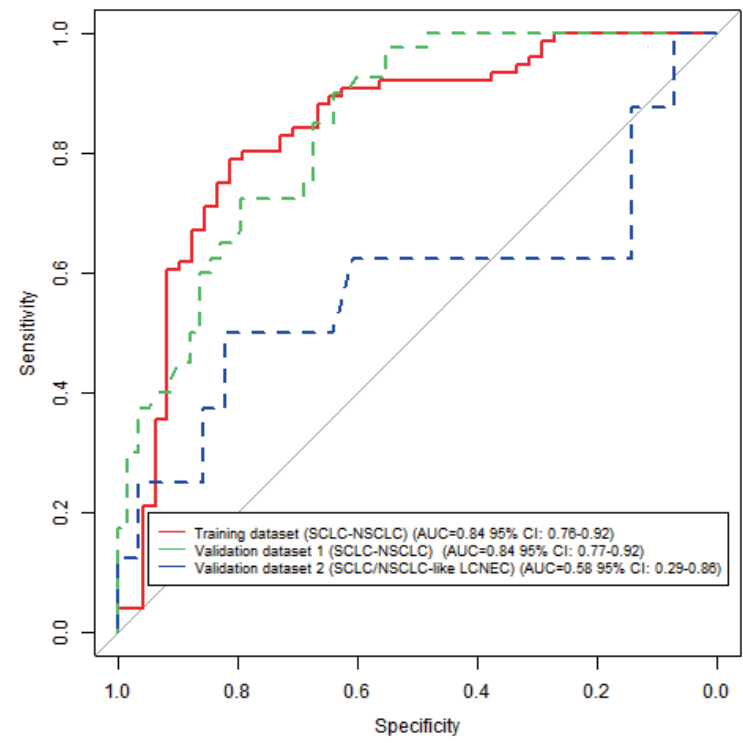

Figure S2.F Receiver operating characteristics curve with area under the curve of training set of CT-scans of SCLC and NSCLC patients, validation set of SCLC and NSCLC patients and the pathological SCLC-like and NSCLC-like LCNEC set. Abbreviations: SCLC = small cell lung carcinoma, NSCLC $=$ non-small cell lung carcinoma, $A U C=$ Area under the curve, $L C N E C=$ large cell neuroendocrine carcinoma. 


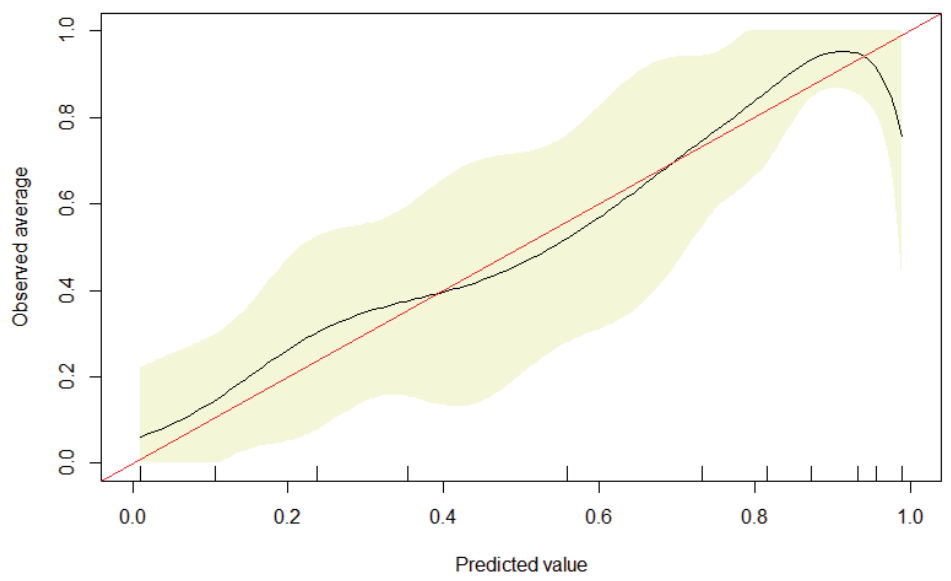

Figure S2.G.1 Calibration plot in training dataset (SCLC/NSCLC) depicting the match between classifiers' probability predictions and actual class probabilities. In yellow the $95 \%$-confidence level belt is plotted. The ticks on the $x$-axis belong to the $x$-coordinate of the individual calibration points.

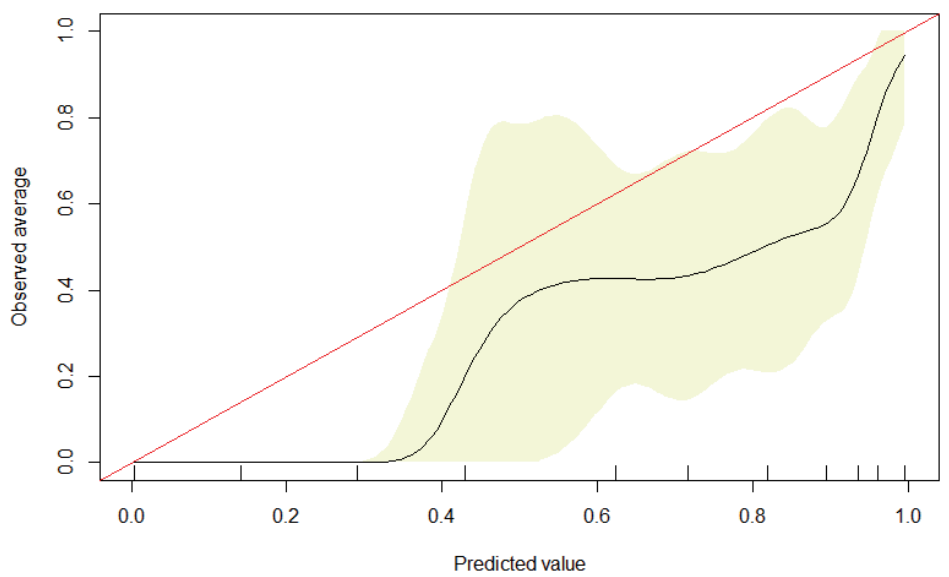

Figure S2.G.2 Calibration plot in first validation set (SCLC/NSCLC) depicting the match between classifiers' probability predictions and actual class probabilities. In yellow the $95 \%$-confidence level belt is plotted. The ticks on the $x$-axis belong to the $x$-coordinate of the individual calibration points. 


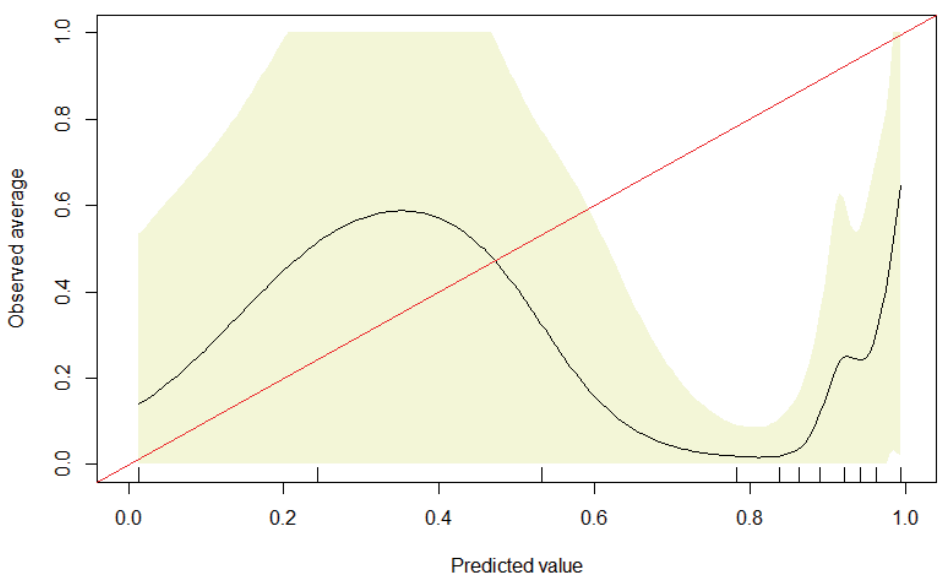

Figure S2.G.3 Calibration plot in second validation dataset (pathological SCLC-like/NSCLC-like LCNEC) depicting the match between classifiers' probability predictions and actual class probabilities. In yellow the $95 \%$-confidence level belt is plotted. The ticks on the $\mathrm{x}$-axis belong to the $\mathrm{x}$ coordinate of the individual calibration points.

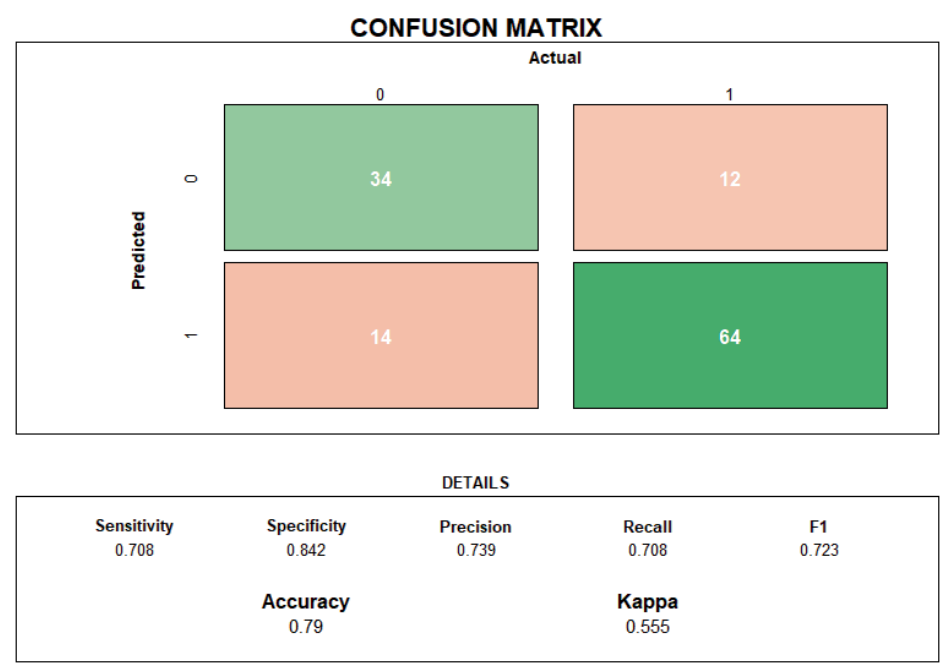

Figure S2.H.1 Confusion matrix in training dataset (SCLC/NSCLC). 


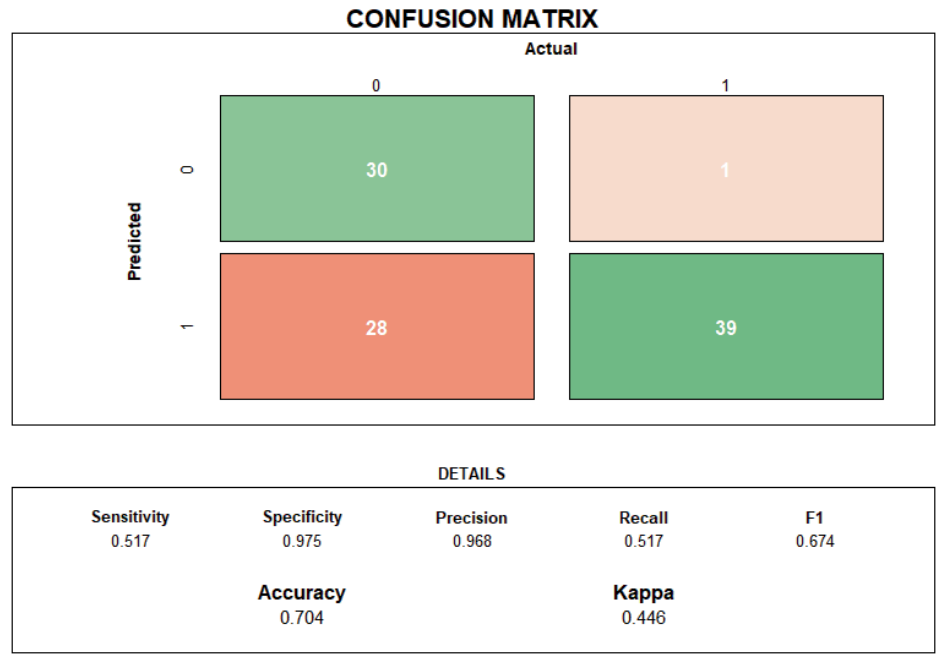

Figure S2.H.2 Confusion matrix in first validation dataset (SCLC/NSCLC).

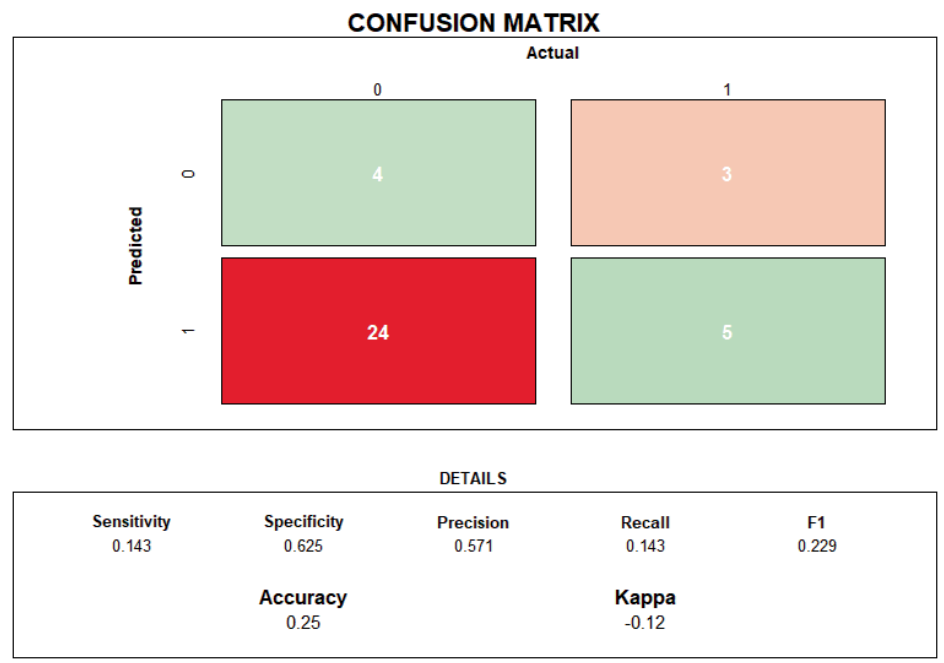

Figure S2.H.3 Confusion matrix in second validation dataset (pathological SCLC-like/NSCLC-like LCNEC). 


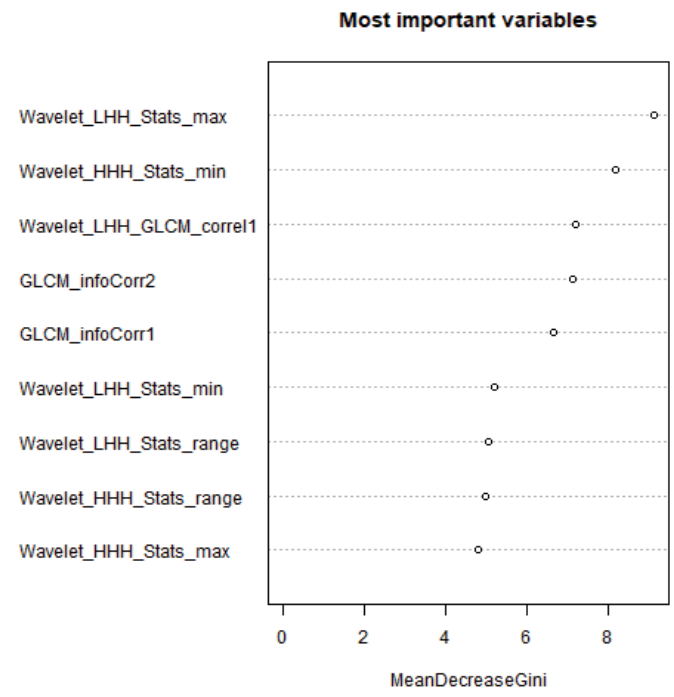

Figure S2.I.1 Features in the model before Combat harmonization and their importance in the model. Mean Decrease in Gini is the average of a variable's total decrease in node impurity, weighted by the proportion of samples reaching that node in each individual decision tree in the random forest. A higher Mean Decrease in Gini indicates higher variable importance.

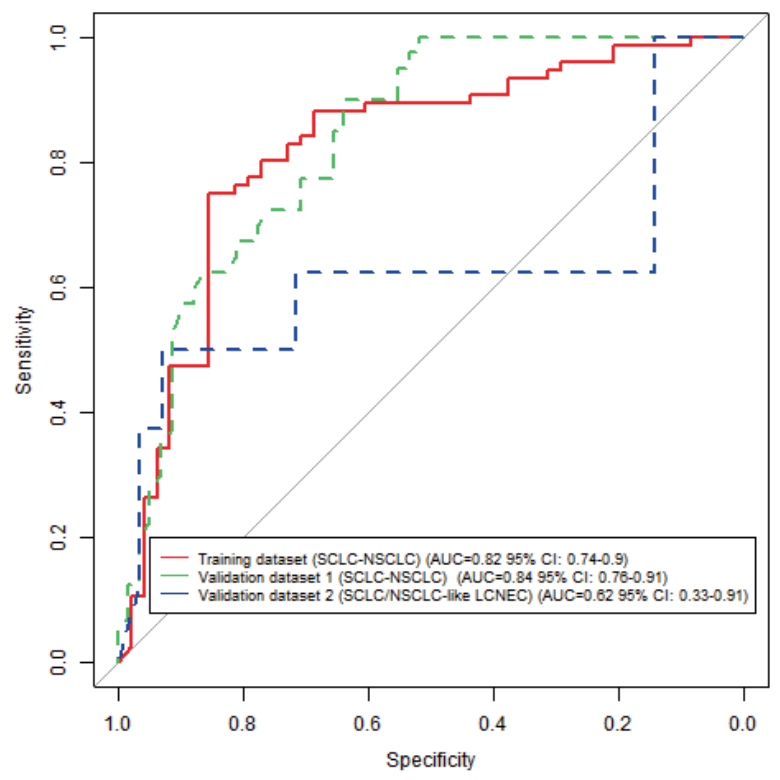

Figure S2.I.2 Receiver operating characteristics curve of the model before Combat harmonization with area under the curve of training set of CT-scans of SCLC and NSCLC patients, validation set 1 of SCLC and NSCLC patients and validation set 2 of the pathological SCLC-like and NSCLC-like LCNEC. Abbreviations: $S C L C=$ small cell lung carcinoma, $N S C L C=$ non-small cell lung carcinoma, $A \cup C=$ Area under the curve, LCNEC = large cell neuroendocrine carcinoma. 
Table S2.A Association between pathological confirmed diagnosis and SCLC-like or NSCLC-like appearance on CT-scans evaluated by pulmonary oncologists and radiologists.

\begin{tabular}{|c|c|c|c|c|c|c|}
\hline & \multicolumn{2}{|c|}{ Controls } & \multirow[b]{2}{*}{ p-value } & \multicolumn{2}{|c|}{ LCNEC } & \multirow[b]{2}{*}{ p-value } \\
\hline & $\begin{array}{l}\text { SCLC } \\
\mathrm{N}(\%) \\
\end{array}$ & $\begin{array}{l}\text { NSCLC } \\
\text { N (\%) }\end{array}$ & & $\begin{array}{c}\text { pSCLC-like } \\
\text { N (\%) }\end{array}$ & $\begin{array}{c}\text { pNSCLC-like } \\
\text { N (\%) }\end{array}$ & \\
\hline Pulmonologists & $\mathrm{N}=10$ & $\mathrm{~N}=10$ & & $\mathrm{~N}=34$ & $\mathrm{~N}=10$ & \\
\hline Survey SCLC-like & $2(100)$ & $0(0)$ & 0.011 & $1(100)$ & $0(0)$ & 1.00 \\
\hline Survey NSCLC-like & $1(13)$ & $7(88)$ & & $15(79)$ & $4(21)$ & \\
\hline Survey NC & $7(70)$ & $3(30)$ & & $18(75)$ & $6(25)$ & \\
\hline Radiologists & $\mathrm{N}=10$ & $\mathrm{~N}=10$ & & $N=31$ & $N=7$ & \\
\hline Survey SCLC-like & $2(100)$ & $0(0)$ & 0.332 & $2(100)$ & $0(0)$ & 1.00 \\
\hline Survey NSCLC-like & $3(33)$ & $6(66)$ & & $7(88)$ & $1(13)$ & \\
\hline Survey NC & $5(56)$ & $4(44)$ & & $22(79)$ & $6(21)$ & \\
\hline
\end{tabular}

Abbreviations: $\mathrm{SCLC}=$ small cell lung carcinoma, NSCLC $=$ non-small cell lung carcinoma, $\mathrm{LCNEC}=$ large cell neuroendocrine carcinoma, pSCLC-like = pathological SCLC-like, pNSCLC-like = pathological NSCLC-like, NC = no consensus between the pulmonary oncologists or radiologists. "Fisher's exact test

Table S2.B Semantic features of stage IV SCLC and NSCLC and pathological SCLC-like and NSCLC-like LCNEC.

\begin{tabular}{|c|c|c|c|c|c|c|}
\hline & \multicolumn{2}{|c|}{ Controls } & \multicolumn{4}{|c|}{ LCNEC } \\
\hline & SCLC & NSCLC & & pSCLC-like & pNSCLC-like & \\
\hline & N (\%) & N (\%) & p-value $\#$ & N (\%) & N (\%) & p-value ${ }^{\#}$ \\
\hline Total number of patients & 10 & 10 & & 31 & 7 & \\
\hline \multicolumn{7}{|l|}{ Tumor location (strict) } \\
\hline Central & $3(30)$ & $0(0)$ & 0.040 & $5(16)$ & $2(29)$ & 0.655 \\
\hline Peripheral & $1(10)$ & $6(60)$ & & $14(45)$ & $2(29)$ & \\
\hline $\mathrm{ND} / \mathrm{NC}$ & $6(60)$ & $4(40)$ & & $12(39)$ & $3(43)$ & \\
\hline \multicolumn{7}{|l|}{ Involved lung lobe } \\
\hline LLL & $1(10)$ & $2(20)$ & 0.398 & $2(7)$ & $2(29)$ & 0.275 \\
\hline LUL & $2(20)$ & $1(10)$ & & $14(45)$ & $2(29)$ & \\
\hline RLL & $3(30)$ & $1(10)$ & & $3(10)$ & $0(0)$ & \\
\hline RML & $0(0)$ & $1(10)$ & & $0(0)$ & $0(0)$ & \\
\hline RUL & $2(20)$ & $5(50)$ & & $7(23)$ & $3(43)$ & \\
\hline ND/ NC & $2(20)$ & $0(0)$ & & $5(16)$ & $0(0)$ & \\
\hline \multicolumn{7}{|l|}{ Tumor size } \\
\hline$<3 \mathrm{~cm}$ & $1(10)$ & $3(30)$ & 0.460 & $4(13)$ & $3(43)$ & 0.064 \\
\hline $3-7 \mathrm{~cm}$ & $5(50)$ & $6(60)$ & & $10(32)$ & $4(57)$ & \\
\hline$>7 \mathrm{~cm}$ & $2(20)$ & $1(10)$ & & $10(32)$ & $0(0)$ & \\
\hline $\mathrm{ND} / \mathrm{NC}$ & $2(20)$ & $0(0)$ & & $7(23)$ & $0(0)$ & \\
\hline \multicolumn{7}{|l|}{$T$} \\
\hline TO & $0(0)$ & $1(10)$ & 0.727 & $3(10)$ & $2(29)$ & 0.724 \\
\hline $\mathrm{T} 1$ & $3(30)$ & $4(40)$ & & $3(10)$ & $0(0)$ & \\
\hline $\mathrm{T} 2$ & $2(20)$ & $0(0)$ & & $8(26)$ & $1(14)$ & \\
\hline T3 & $2(20)$ & $3(3)$ & & $11(36)$ & $3(43)$ & \\
\hline $\mathrm{T} 4$ & $0(0)$ & $0(0)$ & & $0(0)$ & $0(0)$ & \\
\hline $\mathrm{ND} / \mathrm{NC}$ & $3(30)$ & $2(2)$ & & $6(19)$ & $1(14)$ & \\
\hline \multicolumn{7}{|l|}{$\mathbf{N}$} \\
\hline NO & $0(0)$ & $3(30)$ & 0.088 & $3(10)$ & 1 (14) & 1.000 \\
\hline N1 & $1(1)$ & $0(0)$ & & $2(7)$ & $0(0)$ & \\
\hline N2 & $4(40)$ & $5(50)$ & & $9(29)$ & $2(29)$ & \\
\hline N3 & $5(50)$ & $1(10)$ & & $16(52)$ & $4(57)$ & \\
\hline ND/ NC & $0(0)$ & $1(10)$ & & $1(3)$ & $0(0)$ & \\
\hline
\end{tabular}


Table S2.B (continued)

\begin{tabular}{|c|c|c|c|c|c|c|}
\hline & \multicolumn{2}{|c|}{ Controls } & \multicolumn{4}{|c|}{ LCNEC } \\
\hline & $\begin{array}{l}\text { SCLC } \\
\mathrm{N}(\%) \\
\end{array}$ & $\begin{array}{c}\text { NSCLC } \\
\text { N (\%) } \\
\end{array}$ & p-value ${ }^{\#}$ & $\begin{array}{c}\text { pSCLC-like } \\
\text { N (\%) } \\
\end{array}$ & $\begin{array}{c}\text { pNSCLC-like } \\
\text { N (\%) }\end{array}$ & p-value ${ }^{\#}$ \\
\hline \multicolumn{7}{|l|}{ Liver metastases } \\
\hline No & $5(50)$ & $7(70)$ & 0.546 & $16(52)$ & $3(43)$ & 0.182 \\
\hline Limited & $1(10)$ & $0(0)$ & & $3(10)$ & $2(29)$ & \\
\hline Diffuse & $3(30)$ & $1(10)$ & & $4(13)$ & $2(29)$ & \\
\hline $\mathrm{ND} / \mathrm{NC}$ & $1(10)$ & $2(20)$ & & $8(26)$ & $0(0)$ & \\
\hline \multicolumn{7}{|l|}{ Aspect tumor } \\
\hline Homogeneous & $3(30)$ & $2(20)$ & 1.000 & $8(26)$ & $1(14)$ & 1.000 \\
\hline Heterogeneous & $6(60)$ & $7(70)$ & & $19(61)$ & $5(71)$ & \\
\hline $\mathrm{ND} / \mathrm{NC}$ & $1(10)$ & $1(10)$ & & $4(13)$ & $1(14)$ & \\
\hline \multicolumn{7}{|l|}{ Tumor border* } \\
\hline Smooth & $1(10)$ & $1(10)$ & 1.000 & $5(16)$ & $0(0)$ & 0.561 \\
\hline Lobulated & $5(50)$ & $6(60)$ & 1.000 & $16(52)$ & $3(43)$ & 1.000 \\
\hline Spiculated & $4(40)$ & $6(60)$ & 0.656 & $13(42)$ & $4(57)$ & 0.678 \\
\hline \multicolumn{7}{|l|}{ Internal characteristics* } \\
\hline Calcification & $0(0)$ & $0(0)$ & - & $2(7)$ & $1(14)$ & 0.467 \\
\hline Necrosis & $3(30)$ & $5(50)$ & 0.650 & $8(26)$ & $0(0)$ & 0.307 \\
\hline Air bronchogram & $2(20)$ & $2(20)$ & 1.000 & $7(23)$ & $2(29)$ & 1.000 \\
\hline Cavitation & $0(0)$ & $0(0)$ & - & $1(3)$ & $0(0)$ & 1.000 \\
\hline Pleural invasion & $2(20)$ & $1(10)$ & 1.000 & $9(29)$ & $1(14)$ & 0.650 \\
\hline Notching & $0(0)$ & $0(0)$ & - & $0(0)$ & $0(0)$ & - \\
\hline \multicolumn{7}{|c|}{ External characteristics* } \\
\hline Groundglass & $0(0)$ & $2(20)$ & 0.474 & $9(29)$ & $0(0)$ & 0.164 \\
\hline Bubble lucencies & $0(0)$ & $0(0)$ & - & $0(0)$ & $0(0)$ & - \\
\hline Open bronchus sign & $0(0)$ & $0(0)$ & - & $0(0)$ & $0(0)$ & - \\
\hline Pleural tag & $0(0)$ & $3(30)$ & 0.211 & $4(13)$ & $0(0)$ & 1.000 \\
\hline Distal mucus plug & $0(0)$ & $1(10)$ & 1.000 & $1(3)$ & $0(0)$ & 1.000 \\
\hline Distal atelectasis & $2(20)$ & $0(0)$ & 0.474 & $4(13)$ & $1(14)$ & 1.000 \\
\hline Pleural fluid & $1(10)$ & $1(10)$ & 1.000 & $2(7)$ & $0(0)$ & 1.000 \\
\hline Satellite lesions & $2(20)$ & $4(40)$ & 0.628 & $11(36)$ & $3(43)$ & 1.000 \\
\hline Emphysema & $2(20)$ & $4(40)$ & 0.628 & $13(42)$ & $4(57)$ & 0.678 \\
\hline
\end{tabular}

* Multiple answers possible for each scan. Abbreviations: SCLC = small cell lung carcinoma, NSCLC = non-small cell lung carcinoma, IHC = immunohistochemistry, LCNEC = large cell neuroendocrine carcinoma, pSCLC-like = pathological SCLC-like, pNSCLC-like = pathological NSCLC-like, ND $=$ Not determinable (could not be determined by $\geq 2 / 3$ radiologists), $N C=$ No consensus (no majority ( $\geq 2 / 3$ ) for one answer $(3 / 3$ for tumor location)), $L L L=$ left lower lobe, $L U L=$ left upper lobe, $R L L=$ right lower lobe, $R M L=$ right middle lobe, $R U L=$ right upper lobe. "Fisher's exact test. 


\section{Supplemental methods}

\section{Supplemental methods S2.A: Explanation for pulmonary oncologists about the survey}

Please indicate for every CT-scan which diagnosis you think is most suitable (small cell or non-small cell lung carcinoma). In case you have a clear preference for one of the diagnoses, tick this answer, you do not need to be very sure. However, you do not have to gamble either and in case you think both diagnoses could be likely, please tick this answer ('not determinable').

\section{Supplemental methods S2.B: Construction of combination scores}

Imaging interpretation by pulmonary oncologists and radiologists

If $\geq 50 \%$ of pulmonary oncologists/radiologists scored a CT-scan as 'small cell' and none of them scored the same image as 'non-small cell', a combination score 'imaging SCLClike' (iSCLC-like) was assigned. A similar approach was used for NSCLC (iNSCLC-like). In all other cases, the combination score was set to 'no consensus'.

\section{Semantic features}

The feature was scored positive if at least two of the radiologists scored the feature as being present, otherwise the score was considered negative. If the feature was scored differently by the three radiologists in multiple choice questions, the combination score was set to 'no consensus'. If no answer was provided by at least two radiologists, the score was set at 'not determinable'. For tumor location (central/peripheral), full consensus between the 3 radiologists was required for the combination score.

\section{Supplemental methods S2.C: Radiomics workflow}

\section{Image pre-processing, feature extraction, and harmonization}

International Biomarker Standardization Initiative (IBSI)-compliant radiomics features as well as other non-IBSI covered features were extracted with the RadiomiX Discovery Toolbox (version October 2019, supported by Oncoradiomics, Liège, Belgium, https://www.oncoradiomics.com). Houndsfield Unit (HU) intensities beyond -1024 and $+3071 \mathrm{HU}$ were clipped (assigned the value -1024 and +3071 respectively). An image intensity discretization with a fixed bin width of $25 \mathrm{HU}$ was used for feature extraction. Images were resampled to a voxel size of $1 \times 1 \times 5 \mathrm{~mm}^{3}$ using cubic interpolation. This 
'standard' voxel size was chosen according to the highest slice thickness and the median pixel spacing. Radiomics features were extracted consisting of five main groups: 1) fractal features, 2) first order statistics, 3) shape and size, 4) texture descriptors including gray level co-occurrence (GLCM), gray level run-length (GLRLM) and gray level size-zone texture matrices (GLSZM), 5) features from groups 1, 3 and 4 after wavelet decomposition. There were no missing feature values. Definitions and detailed feature descriptions are described elsewhere. ${ }^{1}$

Radiomics feature values are potentially sensitive to inter-scanner model, acquisition protocol and reconstruction settings variation. Therefore, we used The ComBat statistical harmonization technique, initially developed by Johnson et al. for gene expression microarray data (even for small sample sizes), that was recently exploited in multicenter PET, MRI, and CT radiomics studies (Supplemental Figure 2.I). ${ }^{2-4}$ The batch covariate used was the scanner type.

\section{Statistical analysis}

The statistical analysis for model development was performed with R studio software, version 3.3.4 (http://www.R-project.org). The R packages used in this study were caret, missForest, sva and randomForest. The independent samples Mann-Whitney test was used for comparison of unpaired, continuous, non-normally distributed data and the chi-square and Fisher's exact tests were used for the comparison of categorical variables. All reported statistical significance levels were two-sided, with a significance level $<0.05$. The $95 \%$ confidence intervals $(\mathrm{Cl})$ were reported for all relevant model performance metrics. A random forest (RF) machine-learning classifier was computed, with a 10 -fold cross validation treebag recursive feature elimination algorithm (Caret package) loop reshuffled 10 times (outer resampling method whereby features were re-ranked), to classify patients as SCLC/ NSCLC based on the optimal combination of radiomics features. We used recursive feature elimination (RFE) for feature selection. This is a selection method based on the concept of repeated model construction (e.g. RF) to select features according to their performance (e.g. classification error, importance), setting one subset of features aside and then repeating the process with the rest of the features, until all features in the dataset are exhausted. Features are then ranked according to when they were eliminated. As such, RFE is a greedy optimization procedure that tries to find the best performing subset of features.

In order to select the optimal number of features for the final Random Forrest model the features were ranked according to decreasing relative importance in RFE. Gradually these features were added to a multivariable Random Forrest model until the first peak in validation fold accuracy was obtained or after the first peak until the accuracy drops 
by more than 0.01 , depending if there is an oscillation or noise pattern leading to multiple peaks.

\section{References}

1. Aerts HJ, Velazquez ER, Leijenaar RT, et al. Decoding tumour phenotype by noninvasive imaging using a quantitative radiomics approach. Nature communications 2014;5:4006.

2. Johnson WE, Li C, Rabinovic A. Adjusting batch effects in microarray expression data using empirical Bayes methods. Biostatistics (Oxford, England) 2007;8:118-127.

3. Orlhac F, Frouin F, Nioche C, et al. Validation of A Method to Compensate Multicenter Effects Affecting CT Radiomics. Radiology 2019;291:53-59.

4. Lucia F, Visvikis D, Vallieres M, et al. External validation of a combined PET and MRI radiomics model for prediction of recurrence in cervical cancer patients treated with chemoradiotherapy. European journal of nuclear medicine and molecular imaging 2019;46:864-877. 


\section{Chapter 3}

\section{In-depth molecular analysis of combined and co- primary pulmonary large cell neuroendocrine carcinoma and adenocarcinoma}

B.C.M. Hermans, J.L. Derks, L.M. Hillen, I. van der Baan, E.C. van den Broek, J.H. von der Thüsen, R.J. van Suylen, P.N. Atmodimedjo, T.D. den Toom, C. Coumans-Stallinga, PALGA-group, W. Timens, W.N.M. Dinjens, H.J. Dubbink, E.J.M. Speel*, A-M.C. Dingemans*

* contributed equally to this work Submitted 


\section{Chapter 4}

\section{Large cell neuroendocrine carcinoma with a solitary brain metastasis and low Ki-67: \\ a unique subtype}

B.C.M. Hermans, J.L. Derks, H.J.M. Groen, J.A. Stigt, R.J. van Suylen, L.M. Hillen, E.C. van den Broek, E.J.M. Speel*, A-M.C. Dingemans*

* contributed equally to this work Endocr Connect 2019;8(12):1600-1606 


\section{Abstract}

\section{Background}

Stage IV large cell neuroendocrine carcinoma (LCNEC) of the lung generally presents as disseminated and aggressive disease with a Ki-67 proliferation index (PI) 40-80\%. LCNEC can be subdivided in two main subtypes: the first harboring TP53/RB1 mutations (small cell lung carcinoma (SCLC)-like), the second with mutations in TP53 and STK11/KEAP1 (non-small cell lung carcinoma (NSCLC)-like). Here we evaluated 11 LCNEC patients with only a solitary brain metastasis and evaluate phenotype, genotype and follow-up.

\section{Methods}

Eleven LCNEC patients with solitary brain metastases were analyzed. Clinical characteristics and survival data were retrieved from medical records. Pathological analysis included histomorphological analysis, immunohistochemistry (pRb and Ki-67 $\mathrm{PI})$ and next generation sequencing (TP53, RB1, STK11, KEAP1 and MEN1).

\section{Results}

All patients had NO or N1 disease. Median overall survival (OS) was 12 months (95\% confidence interval (CI) 5.5-18.5 months). Mean Ki-67 PI was 59\% (range 15-100\%). In $6 / 11$ LCNEC Ki-67 PI was $\leq 40 \%$. OS was longer for Ki-67 $\leq 40 \%$ compared to $>40 \%$ (17 months (95\% Cl 11-23 months) vs. 5 months (95\% Cl 0.7-9 months), $\mathrm{p}=0.007$ ). Two patients were still alive at follow-up after 86 and 103 months, both had Ki- $67 \leq 40 \%$. $8 / 11$ patients could be subclassified and both SCLC-like $(n=6)$ and NSCLC-like $(n=2)$ subtype were present. No MEN1 mutation was found.

\section{Conclusion}

Stage IV LCNEC with a solitary brain metastasis and NO/N1 disease show in the majority of cases Ki-67 PI $\leq 40 \%$ and prolonged survival, distinguishing them from general LCNEC. This unique subgroup can be both of the SCLC-like and NSCLC-like subtype. 


\section{Introduction}

Neuroendocrine neoplasms can originate in various organ systems and are subdivided in neuroendocrine tumors (NET) and neuroendocrine carcinoma (NEC). ${ }^{1}$ The most common NEC is small cell lung carcinoma (SCLC), followed by pulmonary large cell neuroendocrine carcinoma (LCNEC). ${ }^{2}$ Although LCNEC is the second most frequent NEC, it represents only $1-3 \%$ of all types of lung cancer. ${ }^{3,4}$ Generally, stage IV LCNEC presents with extensive metastatic disease and poor survival rates ( $<10$ months), comparable to SCLC. $^{3,5}$ Furthermore, Ki-67 proliferation index (PI) of LCNEC is approximately in the same range as SCLC (40-80\%) whereas the PI is distinctly lower in well differentiated neuroendocrine tumors such as typical and atypical carcinoid (0-20\%) (Figure 4.1). ${ }^{6}$ Based on mutational analysis, LCNEC can be separated in two main molecular subtypes: the first with mutations in TP53/RB1 (a hallmark of SCLC), the other with mutations in TP53/STK11 and/or KEAP1 genes and retained pRb protein expression (non-small cell lung carcinoma (NSCLC)-like). ${ }^{7,8}$ In addition, a LCNEC subtype with lower Ki-67 PI was identified having a MEN1 mutation and, more recently, a study showed overlapping molecular alterations between atypical carcinoid and LCNEC for TP53, RB1 and MEN1.,9

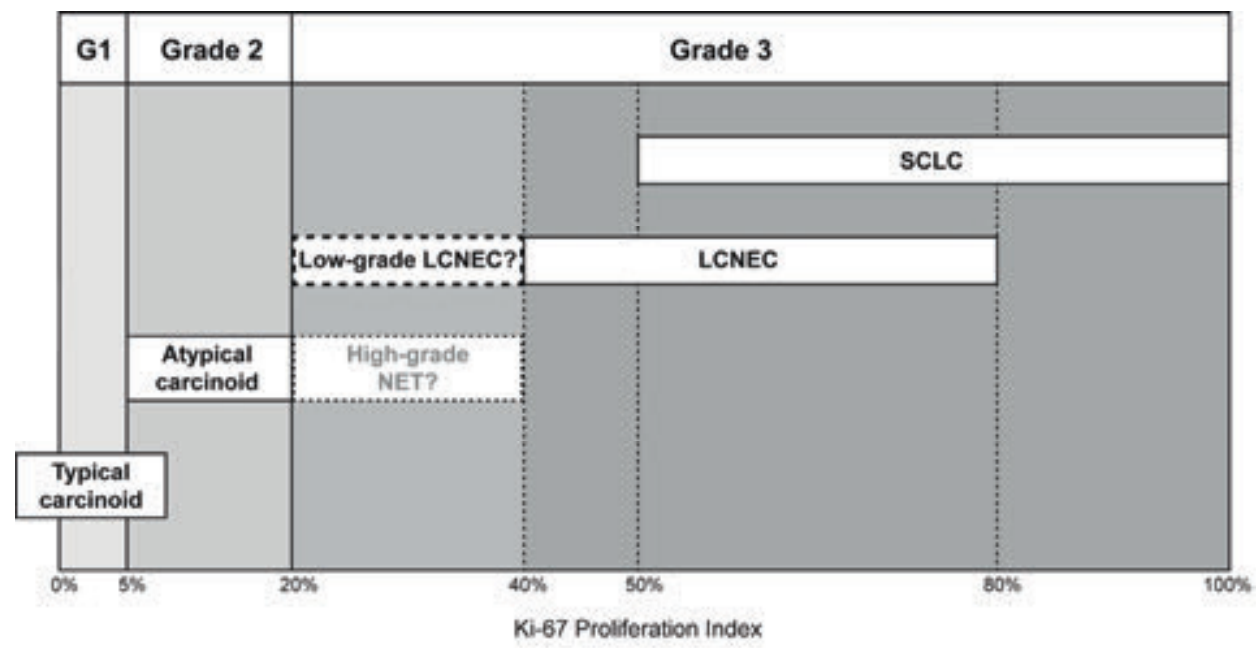

Figure 4.1 Ki-67 proliferation indices (PIs) in the spectrum of pulmonary neuroendocrine neoplasms. Carcinoids have a Ki-67 PI $\leq 20 \%$ whereas LCNEC and SCLC generally have a Ki-67 PI $>40 \%$. The group with $\mathrm{Ki}-67 \mathrm{PI}>20 \%$ and $\leq 40 \%$ might be considered an intermediate NEN group, including high-grade NET and/or low-grade LCNEC, not specified in current WHO-criteria. The majority of LCNEC patients with solitary brain metastases have a Ki-67 PI in this category. Abbreviations: G1 = grade $1, \mathrm{LCNEC}=$ large cell neuroendocrine carcinoma, $\mathrm{NET}=$ neuroendocrine tumor, $\mathrm{SCLC}=$ small cell lung carcinoma 
In contrast to these high grade neuroendocrine carcinomas, a subgroup of NSCLC presents with a solitary metastasis, limited to the brain. This subgroup comprises $7 \%$ of NSCLC and shows prolonged survival compared to NSCLC with extensive metastatic disease. ${ }^{10}$ According to current guidelines, local radical treatment of the lesions may be considered in patients with solitary brain metastases and a good performance score. ${ }^{11}$

In this study, we present a unique subgroup of 11 stage IV LCNEC patients harboring a synchronous solitary brain metastasis as only metastatic site. We hypothesized that those tumors had a lower Ki-67 PI than general LCNEC and that those tumors were of the NSCLC-like molecular subtype. Therefore, tumors were evaluated for Ki-67 PI, $\mathrm{pRb}$ expression and gene mutations.

\section{Methods}

We identified 10 stage IV LCNEC patients who underwent surgical resection of synchronous solitary brain metastases by screening of pathological reports, making use of the nationwide network and registry of histo- and cytopathology in the Netherlands (PALGA, 2003-2012). ${ }^{12,13}$ Furthermore, we identified one additional LCNEC patient treated in our own hospital with lobectomy and stereotactic radiotherapy targeting his solitary brain metastasis (2015). Clinical characteristics and survival data were retrieved from medical records.

All histological samples were centrally reviewed to confirm LCNEC diagnosis according to the criteria described in the World Health Organization (WHO) classification of lung tumors, 2015. ${ }^{14}$ Immunohistochemistry (IHC) was performed with antibodies against Ki67 (Mib-1) and pRb (13A10) as described earlier. ${ }^{13} \mathrm{Ki}-67 \mathrm{PI}$ was assessed semiquantitatively by an experienced pulmonary pathologist (LH) as is done in usual care in our center. ${ }^{15}$ Targeted next generation sequencing (NGS) for TP53, RB1, STK11 and $K E A P 1$ was performed on tumor tissue from available formalin-fixed paraffinembedded (FFPE) blocks of the primary tumor and/or the brain metastasis. ${ }^{13}$ In addition mutational analysis for MEN1 was performed by NGS. ${ }^{13}$

Median overall survival (OS) was evaluated by Kaplan Meier analysis and differences in survival between low and high Ki-67 PI (arbitrary threshold $\leq 40 \%$ vs. $>40 \%$ ) were tested for significance with Log-Rank test. $P<0.05$ was considered significant. 
The study protocol was approved by the medical ethical committee of the Maastricht University Medical Centre (METC azM/UM 14-4-043). The study is performed according to the Dutch "Federa, Human Tissue and Medical Research: Code of conduct for responsible use (2011)" regulations not requiring patients' informed consent.

\section{Results}

Eleven LCNEC patients with a synchronous solitary brain metastasis were included in the analysis (Table 4.1). Mean age at diagnosis was 59 years (range 34-72), 9/11 patients were male. For five patients, smoking history was available and mean packyears exceeded 40 years. Seven out of 11 patients had NO disease, the other four patients had N1 disease (4/11). Nine out of 11 patients were treated with definitive therapy. Seven of those patients underwent lobectomy/ pneumonectomy and surgical resection of the brain metastasis with all resection margins histopathologically free of tumor cells (Table 4.1: patients $A-E, J$ and $K$ ). Of the other two patients with definitive therapy, one underwent metastasectomy and stereotactic radiotherapy + chemotherapy for the primary tumor $(G)$. The other one underwent a lobectomy and stereotactic radiotherapy for his metastasis (F).

Mean Ki-67 PI was 59\% (range 15-100\%, Table 4.2). In 6/11 LCNEC Ki-67 PI was $\leq 40 \%$. Both tumors with a low Ki-67 PI of $15 \%$ were diagnosed as LCNEC because of the presence of necrosis and a mitotic index of 14 and $>30$ per 10 high power fields, respectively (patients $\mathrm{F}$ and $\mathrm{H}$ ). The patients had a median OS of 12 months (95\% Confidence Interval (CI) 5.5-18.5 months). A significant prolonged OS was seen in patients with a $\mathrm{Ki}-67 \mathrm{PI} \leq 40 \%$ compared to $>40 \%$ (17 months (95\% Cl 11.0-23.0 months) vs. 5 months ( $95 \% \mathrm{Cl}$ 0.7-9.3 months), $\mathrm{p}=0.007$; Figure 4.2). Two patients were still alive after 5 years, a remarkable longer time than average in stage IV LCNEC patients (Table 4.1 and 4.2: patients $G$ and $K$ ). A male patient of 58 years with T2NO disease who

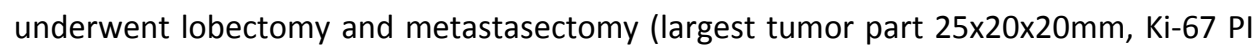
$30 \%$ ), had pulmonary recurrence after 51 months but was still alive at follow-up after 103 months. A woman of 34 years with T1N0 disease underwent a metastasectomy (two parts of tumor tissue, cross sections $8 \mathrm{~mm}$ and $22 \mathrm{~mm}$, Ki-67 PI 40\%) and was treated with chemotherapy and radiotherapy for the primary tumor. She was still alive after 86 months of follow-up, without recurrence of disease. 


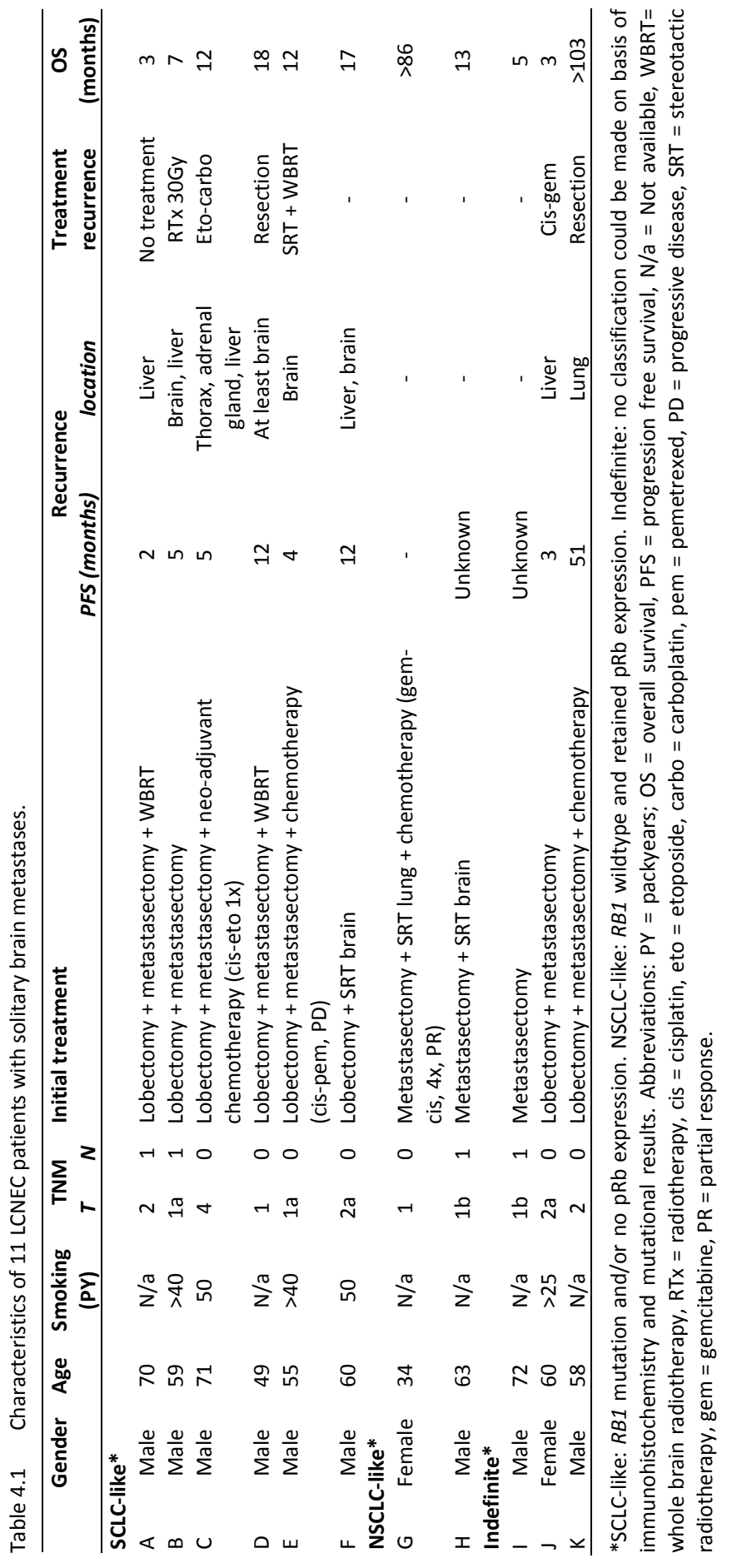


Table 4.2 Mutational and immunohistochemical characteristics of 11 LCNEC patients with solitary brain metastases.

\begin{tabular}{|c|c|c|c|c|c|c|c|}
\hline & \multirow{2}{*}{$\begin{array}{l}\text { OS } \\
\text { (months) }\end{array}$} & \multicolumn{4}{|c|}{ Immunohistochemistry } & \multicolumn{2}{|c|}{ Mutational status } \\
\hline & & $\begin{array}{c}\text { Ki-67 PI } \\
\text { prim }\end{array}$ & $\begin{array}{c}\text { Ki-67 PI } \\
\text { meta }\end{array}$ & $\begin{array}{l}\text { pRb } \\
\text { prim }\end{array}$ & $\begin{array}{c}p R b \\
\text { meta }\end{array}$ & Primary & Metastasis \\
\hline \multicolumn{8}{|c|}{ SCLC-like* } \\
\hline A & 3 & $\mathrm{~N} / \mathrm{a}$ & $90 \%$ & neg & neg & TP53/RB1 & TP53/RB1 \\
\hline B & 7 & $40 \%$ & $40 \%$ & neg & neg & $R B 1$ & $R B 1$ \\
\hline C & 12 & $90 \%$ & $\mathrm{~N} / \mathrm{a}$ & neg & neg & TP53/RB1 & $T P 53 / R B 1$ \\
\hline D & 18 & $\mathrm{~N} / \mathrm{a}$ & $30 \%$ & neg & neg & TP53/RB1 & TP53/RB1 \\
\hline$E$ & 12 & $90 \%$ & $80 \%$ & neg & $\mathrm{N} / \mathrm{a}$ & TP53 & TP53 \\
\hline $\mathrm{F}$ & 17 & $15 \%$ & $\mathrm{~N} / \mathrm{a}$ & neg & $\mathrm{N} / \mathrm{a}$ & TP53 & N/a \\
\hline \multicolumn{8}{|c|}{ NSCLC-like* } \\
\hline G & $>86$ & $\mathrm{~N} / \mathrm{a}$ & $40 \%$ & $\mathrm{~N} / \mathrm{a}$ & pos & $\mathrm{N} / \mathrm{a}$ & TP53/STK11/ KEAP1 \\
\hline $\mathrm{H}$ & 13 & $\mathrm{~N} / \mathrm{a}$ & $15 \%$ & $\mathrm{~N} / \mathrm{a}$ & pos & $\mathrm{N} / \mathrm{a}$ & TP53 \\
\hline \multicolumn{8}{|c|}{ Indefinite* } \\
\hline । & 5 & $\mathrm{~N} / \mathrm{a}$ & $100 \%$ & $\mathrm{~N} / \mathrm{a}$ & pos & $\mathrm{N} / \mathrm{a}$ & TP53/RB1 \\
\hline J & 3 & $90 \%$ & $70 \%$ & neg & neg & KEAP1 & KEAP1 \\
\hline $\mathrm{K}$ & $>103$ & $\mathrm{~N} / \mathrm{a}$ & $30 \%$ & $\mathrm{~N} / \mathrm{a}$ & neg & TP53/KEAP1 & TP53 (different)/ RB1/ KEAP1/STK11 \\
\hline
\end{tabular}

*SCLC-like: RB1 mutation and/or no pRb expression. NSCLC-like: RB1 wildtype and retained pRb expression. Indefinite: no classification could be made on basis of immunohistochemistry and mutational results. OS = overall survival, $\mathrm{Ki}-67 \mathrm{PI}=\mathrm{Ki}-67$ proliferation index, prim = primary tumor, meta $=$ metastatic lesion, $\mathrm{N} / \mathrm{a}=\mathrm{Not}$ available, neg $=$ negative, pos $=$ positive.

Tissue material of all patients was examined with IHC and NGS (Table 4.2, Supplemental Table S4.A). In seven patients samples from both primary tumors and brain lesions were available. Four LCNEC patients (A-D) had a RB1 (and TP53) mutation with loss of pRb protein expression in IHC analysis, classifying as SCLC-like subtype. Two LCNEC patients ( $E$ and F) had a TP53 mutation in combination with loss of pRb expression and were therefore also regarded as SCLC-subtype. Absence of RB1 mutation and retained $\mathrm{pRb}$ expression was observed in two LCNEC patients ( $\mathrm{G}$ and $\mathrm{H}$ ), classifying them as NSCLC-like subtype. Both NSCLC-like tumors had low Ki-67 PI (40\% and 15\%, respectively). One $L C N E C$ (I) had a $R B 1$ mutation, but retained $\mathrm{pRb}$ expression. Another tumor (J) was RB1 wildtype and had a KEAP1 mutation, but showed also loss of $\mathrm{pRb}$ expression. The last LCNEC (K) had KEAP1 and TP53 mutations in the primary tumor (no $\mathrm{pRb}$ available) and additional STK11 and RB1 mutations as well as a different TP53 mutation in the metastatic lesion. Therefore, those last three tumors could not definitely be classified as SCLC-like or NSCLC-like LCNEC. No MEN1 mutations were identified in the LCNEC cases. 


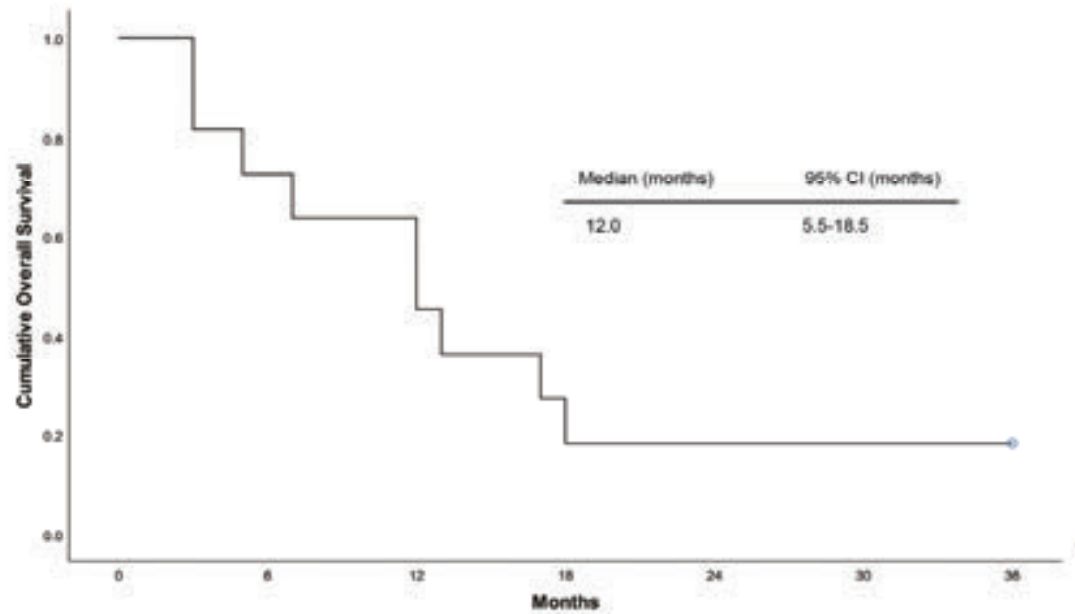

A

Number of patients at risk

\begin{tabular}{|l|l|l|l|l|l|l|l|}
\hline LCNEC & 11 & 8 & 5 & 2 & 2 & 2 & 2 \\
\hline
\end{tabular}

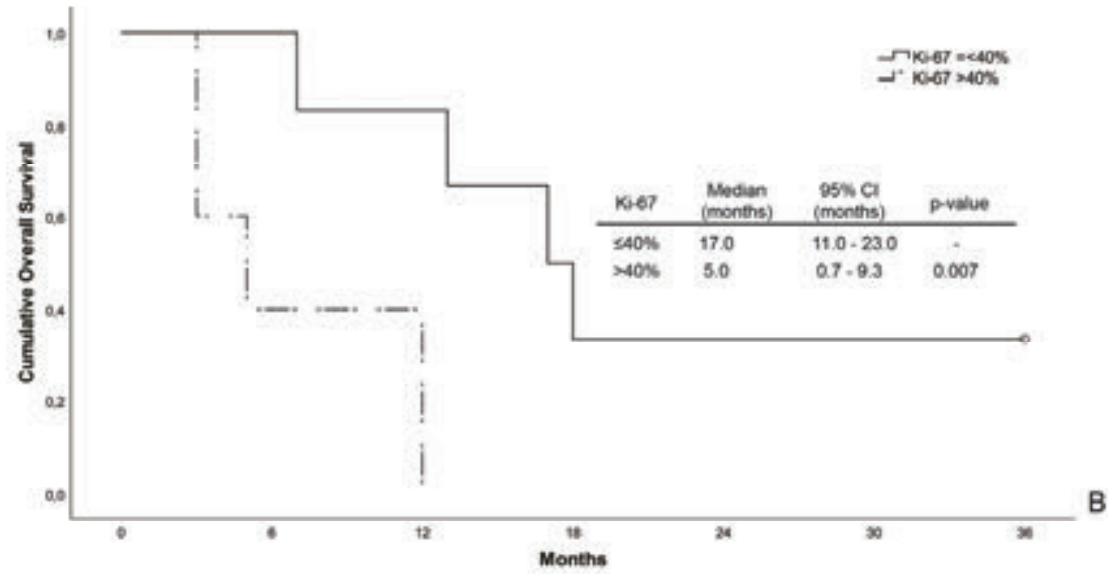

Number of patients at risk

\begin{tabular}{|l|l|l|l|l|l|l|l|}
\hline$\kappa-67540 \%$ & 6 & 6 & 5 & 2 & 2 & 2 & 2 \\
\hline$K-67 \times 40 \%$ & 5 & 2 & 0 & 0 & 0 & 0 & 0 \\
\hline
\end{tabular}

Figure 4.2 A) Overall Survival of LCNEC patients with solitary brain metastases (censored at 36 months). B) Overall Survival of LCNEC patients with solitary brain metastases, exhibiting a Ki-67 proliferation index $\leq 40 \%$ or $>40 \%$ in the primary tumor and/or metastasis (censored at 36 months). 


\section{Discussion}

We here present clinical and molecular features of a unique Dutch multicenter cohort of 11 LCNEC patients with synchronous solitary brain metastases. Whereas the majority of stage IV LCNEC patients endure an aggressive disease, this subgroup presents with limited disease and a relatively low Ki-67 PI. Stage IV LCNEC thus is a heterogeneous disease.

In this series, OS was 12 months and two long term survivors ( $>5$ years) were observed. On the contrary, stage IV LCNEC generally presents as disseminated disease with limited survival time. ${ }^{3,5}$ So far, only few series including oligometastatic LCNEC patients have been reported and this is the first series describing solely LCNEC patients with solitary brain metastases. ${ }^{16,17}$ Furthermore, only a minority of patients with stage IV LCNEC present with N0/N1 disease. In our recent study, $27 \%$ of patients had N0/N1 disease (extracted from ${ }^{13}$ ). Remarkably, in this series of patients with solitary metastases, $64 \%$ of patients have N0 disease and $36 \%$ N1 disease.

The prolonged survival of patients in this study with a Ki-67 PI $\leq 40 \%$ suggests that Ki-67 PI might be used as a prognostic factor in LCNEC patients with solitary brain metastases. A prognostic role for $\mathrm{Ki}-67 \mathrm{PI}$ has already been shown in pulmonary neuroendocrine neoplasms, specifically separating favorable subgroups with Ki-67 PI $<25 \%$ versus $\geq 25 \%$. ${ }^{18}$ The current WHO guideline for lung cancer does not include Ki-67 PI for classification of neuroendocrine neoplasms. ${ }^{14}$ However, Ki-67 PI has been shown to be $\leq 20 \%$ for pulmonary NET and $>40 \%$ for NEC. ${ }^{6}$ Although the mean value of Ki-67 PI in this study was $59 \%$ and therefore falls within the NEC category, the majority of the patients had a $\mathrm{Ki}-67 \mathrm{PI} \leq 40 \%$. This implicates that a subgroup of neuroendocrine neoplasms with a $\mathrm{Ki}-67 \mathrm{PI}>20 \%$ but $\leq 40 \%$ does exist (Figure 4.1 ). This subgroup might comprise high-grade NET, which has been recently described in several studies although not recognized in current WHO classification. ${ }^{19-21}$ However, in those series, these tumors had a carcinoid morphology and absence of TP53 and RB1 mutations. In contrast, in our study all patients had LCNEC morphology and all exhibited TP53 and/or $R B 1$ mutations or loss of $\mathrm{pRb}$ expression but no MEN1 mutations. Therefore, the patients in this study more likely comprise low-grade LCNEC with a Ki-67 PI $>20 \%$ but $\leq 40 \%$ (Figure 4.1 ).

Since the solitary metastatic state is clinically more comparable to NSCLC than to SCLC, we hypothesized that most LCNEC patients with solitary metastases would be of the NSCLC-like subtype. However, six patients were classified as SCLC-like and only two as 
NSCLC-like. The remaining three patients could not definitively be subclassified. Interestingly, mutations were identical in six out of seven patients with available samples of both primary tumor and metastatic lesion. This suggests that mutation of TP53, RB1 and/or STK11/KEAP1 occurs prior to tumor cell dissemination in LCNEC. ${ }^{8}$ In one patient, a TP53 and KEAP1 mutation was found in the primary and metastatic lesion, whereas another TP53 and additional RB1 and STK11 mutations were also found in the metastasis. This suggests that primary and metastatic lesions of this patient were clonally related and additional mutations in the metastasis probably developed later in tumorigenesis. Mutational characteristics have not been reported before for LCNEC patients with solitary brain metastases or oligometastatic disease.

Nine of 11 patients in this series were treated with definitive therapy (resection or stereotactic radiotherapy) for both primary and metastatic lesions, instead of standard treatment for stage IV LCNEC with palliative chemotherapy. Retrospective studies in NSCLC with solitary brain metastases have shown extended OS in patients treated with definitive therapy for primary and metastatic tumors. ${ }^{22}$ No data regarding definitive therapy is available for solitary metastases in SCLC and LCNEC. However, limited data on this subject is available for oligometastatic SCLC and LCNEC, revealing prolonged OS after definitive therapy. ${ }^{17,23}$ Since retrospective datasets are prone to confounding by indication, prospective randomized trials are necessary to confirm the effect of definitive local treatment.

In conclusion, we present 11 LCNEC patients with a solitary brain metastasis and relatively low $\mathrm{Ki}-67 \mathrm{PI}$ in the majority of the patients. Although presence of solitary brain metastases resembles NSCLC more than SCLC, presence of a solitary metastasis was not restricted to NSCLC-like LCNEC. Our data indicate that stage IV LCNEC is a heterogeneous disease, not justifying standard treatment with palliative chemotherapy in all patients. Instead, in those patients a curative treatment strategy for primary and metastatic lesions might be considered to improve OS, especially in LCNEC with relatively low Ki-67 PI. 


\section{References}

1. Rindi G, Klimstra DS, Abedi-Ardekani B, et al. A common classification framework for neuroendocrine neoplasms: an International Agency for Research on Cancer (IARC) and World Health Organization (WHO) expert consensus proposal. Mod Pathol 2018;31:1770-1786.

2. Korse CM, Taal BG, van Velthuysen ML, et al. Incidence and survival of neuroendocrine tumours in the Netherlands according to histological grade: experience of two decades of cancer registry. Eur J Cancer 2013;49:1975-1983.

3. Derks JL, Hendriks LE, Buikhuisen WA, et al. Clinical features of large cell neuroendocrine carcinoma: a population-based overview. Eur Respir J 2016;47:615-624.

4. Takei H, Asamura $H$, Maeshima A, et al. Large cell neuroendocrine carcinoma of the lung: a clinicopathologic study of eighty-seven cases. J Thorac Cardiovasc Surg 2002;124:285-292.

5. Asamura $\mathrm{H}$, Kameya $\mathrm{T}$, Matsuno $\mathrm{Y}$, et al. Neuroendocrine neoplasms of the lung: a prognostic spectrum. J Clin Oncol 2006;24:70-76.

6. Pelosi G, Rindi G, Travis WD, et al. Ki-67 antigen in lung neuroendocrine tumors: unraveling a role in clinical practice. J Thorac Oncol 2014;9:273-284.

7. Rekhtman N, Pietanza MC, Hellmann MD, et al. Next-Generation Sequencing of Pulmonary Large Cell Neuroendocrine Carcinoma Reveals Small Cell Carcinoma-like and Non-Small Cell Carcinoma-like Subsets. Clin Cancer Res 2016;22:3618-3629.

8. George J, Walter V, Peifer M, et al. Integrative genomic profiling of large-cell neuroendocrine carcinomas reveals distinct subtypes of high-grade neuroendocrine lung tumors. Nat Commun 2018;9:1048.

9. Simbolo M, Barbi S, Fassan M, et al. Gene expression profiling of lung atypical carcinoids and large cell neuroendocrine carcinomas identifies three transcriptomic subtypes with specific genomic alterations. J Thorac Oncol 2019.

10. Torok JA, Gu L, Tandberg DJ, et al. Patterns of Distant Metastases After Surgical Management of NonSmall-cell Lung Cancer. Clin Lung Cancer 2017;18:e57-e70.

11. Planchard D, Popat S, Kerr K, et al. Metastatic non-small cell lung cancer: ESMO Clinical Practice Guidelines for diagnosis, treatment and follow-up. Ann Oncol 2018;29:iv192-iv237.

12. Casparie M, Tiebosch AT, Burger $G$, et al. Pathology databanking and biobanking in The Netherlands, a central role for PALGA, the nationwide histopathology and cytopathology data network and archive. Cell Oncol 2007;29:19-24.

13. Derks JL, Leblay N, Thunnissen E, et al. Molecular Subtypes of Pulmonary Large-cell Neuroendocrine Carcinoma Predict Chemotherapy Treatment Outcome. Clin Cancer Res 2018;24:33-42.

14. Travis WD, Brambilla E, Burke AP, et al. WHO Classification of Tumours of the Lung, Pleura, Thymus and Heart Lyon, France: International Agency for Research on Cancer; 2015.

15. Warth A, Fink L, Fisseler-Eckhoff A, et al. Interobserver agreement of proliferation index (Ki-67) outperforms mitotic count in pulmonary carcinoids. Virchows Arch 2013;462:507-513.

16. Kotecha R, Zimmerman A, Murphy ES, et al. Management of Brain Metastasis in Patients With Pulmonary Neuroendocrine Carcinomas. Technol Cancer Res Treat 2016;15:566-572.

17. Naidoo J, Santos-Zabala ML, Iyriboz T, et al. Large Cell Neuroendocrine Carcinoma of the Lung: ClinicoPathologic Features, Treatment, and Outcomes. Clin Lung Cancer 2016;17:e121-e129.

18. Rindi G, Klersy C, Inzani F, et al. Grading the neuroendocrine tumors of the lung: an evidence-based proposal. Endocr Relat Cancer 2014;21:1-16.

19. Rekhtman N, Desmeules P, Litvak AM, et al. Stage IV lung carcinoids: spectrum and evolution of proliferation rate, focusing on variants with elevated proliferation indices. Mod Pathol 2019.

20. Quinn AM, Chaturvedi A, Nonaka D. High-grade Neuroendocrine Carcinoma of the Lung With Carcinoid Morphology: A Study of 12 Cases. Am J Surg Pathol 2017;41:263-270.

21. Vivero M, Scholl LM. "Borderline" neuroendocrine carcinomas of the lung are clinically and genomically distinct from large cell neuroendocrine carcinoma. Mod Pathol 2016;29.

22. Li D, Zhu X, Wang $H$, et al. Should aggressive thoracic therapy be performed in patients with synchronous oligometastatic non-small cell lung cancer? A meta-analysis. J Thorac Dis 2017;9:310-317. 
Chapter 4

23. Xu LM, Cheng C, Kang M, et al. Thoracic radiotherapy (TRT) improved survival in both oligo- and polymetastatic extensive stage small cell lung cancer. Sci Rep 2017;7:9255. 


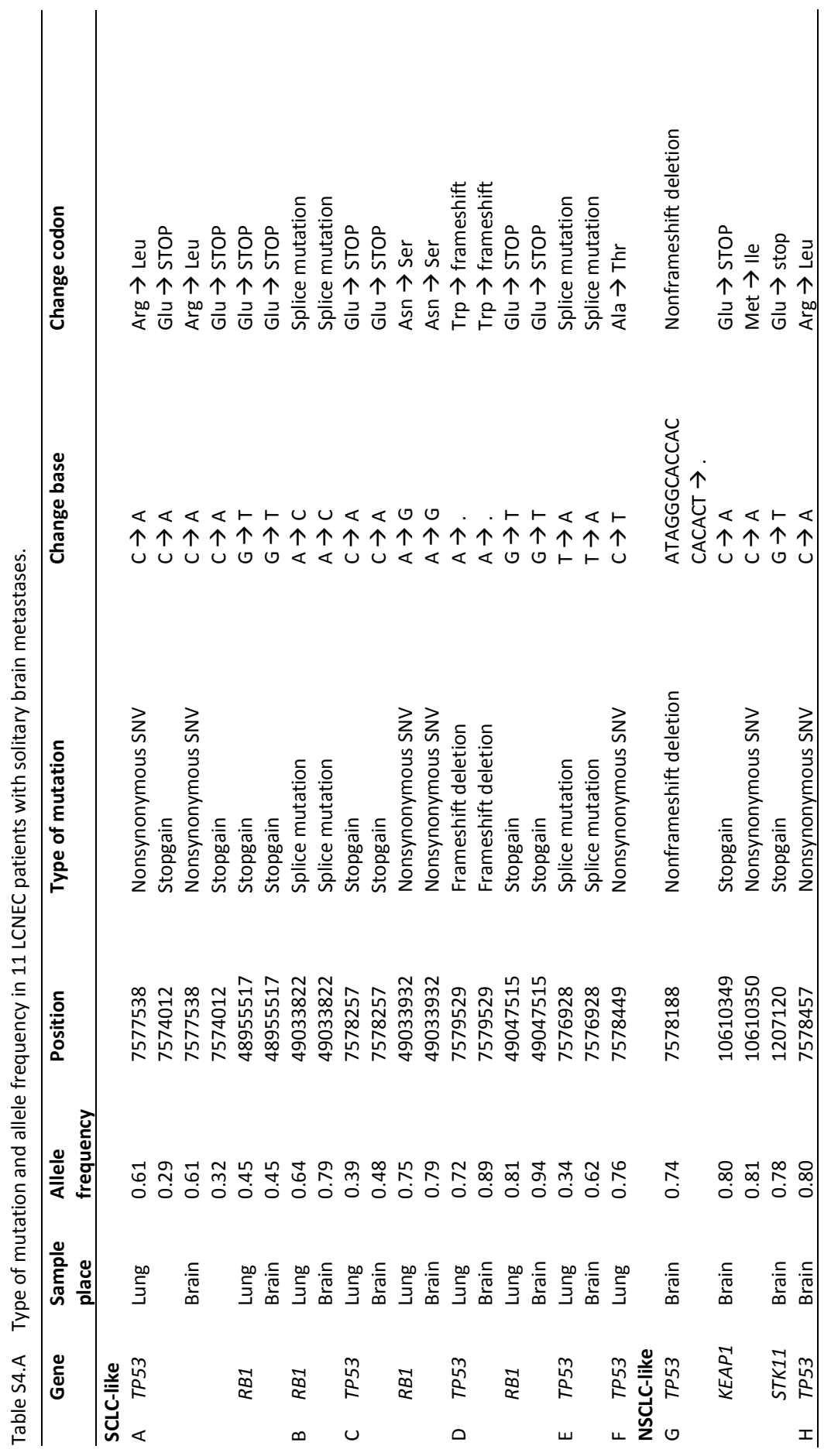




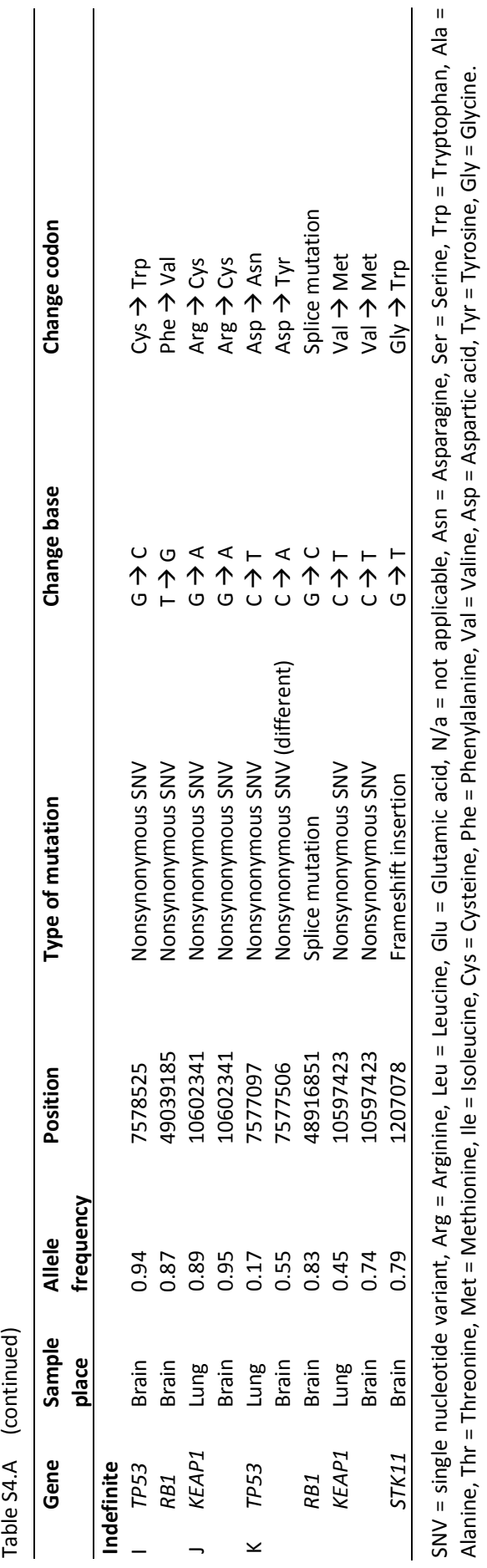




\title{
Chapter 5
}

\begin{abstract}
Pulmonary neuroendocrine neoplasms with well differentiated morphology and high proliferative activity: illustrated by a case series and review of the
\end{abstract}

literature

B.C.M. Hermans, J.L. Derks, L. Moonen, C.H.J. Habraken, J. von der Thüsen, L.M. Hillen, E.J.M. Speel*, A-M.C. Dingemans*

* contributed equally to this work Lung Cancer 2020;150:152-158 


\section{Abstract}

\section{Background}

Pulmonary neuroendocrine neoplasms (NENs) are subdivided in carcinoids and neuroendocrine carcinomas (small cell lung carcinoma and large cell neuroendocrine carcinoma (LCNEC)), based on the presence of necrosis and mitotic index (MI). However, it is unclear if tumors with well differentiated morphology but high proliferation rate should be regarded as LCNEC or as high grade carcinoids. In previous case series, a longer overall survival then expected in LCNEC has been suggested. We describe 7 of those cases analyzed for $\mathrm{pRb}$ expression and overall survival.

\section{Methods}

Cases with well differentiated morphology, but MI $>10 / 2 \mathrm{~mm}^{2}$ and/or Ki-67 proliferation index $>20 \%$ were selected based on pathology reports of consecutive NENs in our university medical center (Maastricht UMC+, 2007-2018) and confirmed by pathological review. Immunohistochemistry was performed to assess pRb expression.

\section{Results}

Seven stage IV cases were included in this study. Median overall survival was 8 months (95\% confidence interval 5-11 months). Cases with well differentiated morphology and preserved pRb expression (4/7) had a median overall survival of 45 months.

\section{Conclusion}

A subgroup of pulmonary NENs with well differentiated morphology but high proliferation rate likely exists. pRb staining might be helpful to predict prognosis, but clinical relevance remains to be studied. 


\section{Introduction}

Neuroendocrine neoplasms (NENs) represent a rare group of heterogeneous tumors which are characterized by a neuroendocrine morphology and expression of neuroendocrine markers. ${ }^{1,2}$ NENs may develop in various organ systems including the lungs. ${ }^{1}$ According to the current World Health Organization (WHO) classification system (2015), pulmonary NENs can be subdivided in two types of neuroendocrine tumors (NETs) and two types of neuroendocrine carcinomas (NECs) based on presence of necrosis and mitotic index (MI). ${ }^{2}$ NETs are subdivided into typical carcinoids (TC) and atypical carcinoids (AC) and are generally well differentiated. TC have no necrosis and a $\mathrm{MI}<2 / 2 \mathrm{~mm}^{2}$. AC are defined by no or dotlike necrosis and a $\mathrm{MI}$ of 2 up to $10 / 2 \mathrm{~mm}^{2}$. NECs can be subdivided in large cell neuroendocrine carcinoma (LCNEC) and small cell neuroendocrine carcinoma (SCLC) and are generally poorly differentiated. Both types are characterized by (abundant) necrosis and a $\mathrm{MI}>10 / 2 \mathrm{~mm}^{2}$, but can be separated based on cell size and amount of cytoplasm. ${ }^{2}$ NECs typically present as aggressive disease with poor survival rates and a median overall survival (OS) of 4-9 months in stage IV LCNEC. In contrast, AC and especially TC behave less aggressively and for the less frequent cases of metastatic carcinoids a median OS up to 58 months has been reported. $^{2-7}$

The current WHO classification relies on evaluation of neuroendocrine differentiation, mitotic rate and necrosis; however, some pulmonary NENs show aspects of both carcinoid and LCNEC, e.g. well differentiated morphology but a $\mathrm{MI}>10 / 2 \mathrm{~mm}^{2}$. Using the WHO guidelines, such tumors are classified as LCNEC, but their clinical behavior might be more comparable to that of carcinoids. ${ }^{8-14}$ In gastrointestinal NENs a group of tumors with preserved morphology but higher proliferation rate was recently identified as NET grade $3 .^{15,16}$ The biologic behavior of comparable pulmonary NENs, straddling the divide between LCNEC and carcinoids, is so far unclear.

Molecular markers might be helpful in prognostically relevant subclassification of neoplasms in case morphology is inconclusive. For example, in gastrointestinal and pancreatic NENs, mutations in MEN1, DAXX and ATRX may support the classification of NET, whereas TP53 and RB1 mutations do so for NEC. ${ }^{15,16}$ In analogy, mutational analysis of pulmonary NENs has shown that RB1 and TP53 are frequently co-mutated in LCNEC/SCLC, but not in carcinoids. ${ }^{10,17-20}$ Inactivation of the $R B 1$ gene is reflected by loss of immunohistochemical (IHC) staining of pRb. ${ }^{17}$ Normal p53 staining has an admixture of negative, weakly and strongly positive cells, whereas a TP53 mutation can 
be reflected by total loss of p53 (null mutations) or diffusely strong positive staining (missense mutations). ${ }^{21-23}$

The aim of this study is to evaluate the pathological and clinical characteristics of pulmonary NENs with well differentiated or equivocal morphology but high proliferation rate and to determine possible clinical relevance. Furthermore, we give an overview of available literature on this special subgroup of pulmonary NENs.

\section{Methods}

\section{Patient selection}

For this retrospective study, patients diagnosed and/or treated with pulmonary NENs (except SCLC) at the Maastricht University Medical Center (MUMC+) between 2007 and 2018 were included. Patient and tumor characteristics and data on treatment and survival were obtained from medical records. All data were recorded in a database program with automatic anonymization (Castor EDC, 2019). This study was approved by the medical ethical review committee and the board of directors of MUMC+ (METC 2019-0970, approval date: 05-04-2019).

\section{Selection of borderline tumors}

Cases were identified by review of the pathology reports of consecutive pulmonary NEN patients with focus on morphology, necrosis, mitotic count and Ki-67 PI. Cases were included if the initial pathologist expressed his or her doubt regarding the subclassification in the conclusion of the report, i.e. no clear distinction could be made between a diagnosis of LCNEC or carcinoid. Furthermore, cases were included if characteristics of both carcinoid and NEC were present in the sample (i.e. well differentiated morphology but mitotic count >10/2 $\mathrm{mm}^{2}$ and/or Ki-67 Pl>20\%).

\section{Pathological review}

Two experienced pulmonary pathologists ( $\mathrm{LH}$ and JvdT) reviewed all selected cases. In accordance with WHO criteria, the diagnosis of pulmonary NEN was confirmed if neuroendocrine morphology was present and for LCNEC the sample stained positive for at least one out of three neuroendocrine markers (chromogranin A, synaptophysin and Cd56). After confirmation of neuroendocrine origin, cases were scored for: 1) differentiation grade (well differentiated, poorly differentiated or equivocal 
differentiation), 2) amount of necrosis (no necrosis, focal necrosis, abundant necrosis), 3) mitotic count per $2 \mathrm{~mm}^{2}$ and 4) Ki-67 PI. The distinction between well differentiated and poorly differentiated morphology was based on architectural features and cellular characteristics. ${ }^{2,24}$ A structured architecture with rosettes, organoid nesting, trabeculae, regular peri- and intratumoral vascular configuration, peripheral palisading and scarcity of desmoplastic stroma was considered as well differentiated histopathology. A less structured architecture with organoid nesting and peripheral palisading but only rudimental rosette-like structures and trabeculae argued for a poorly differentiated morphology. On cytopathologic grounds, uniform, ovoid or round shaped nuclei were considered an indicator for well differentiated morphology, whereas heterogeneous nuclei with spindle like shapes were considered to be poorly differentiated. Furthermore, abundant finely granular cytoplasm was regarded as a sign for well differentiated morphology. In contrast, a less fine cytoplasmic pattern and presence of nuclear molding were assigned to a poorly differentiated morphology. Cases with predominant characteristics of well differentiated morphology, but also some characteristics of poorly differentiated morphology were classified as 'equivocal' and included in the study. In case the pathologists initially did not agree about well differentiated or poorly differentiated morphology, a decision was made based upon consensus. Mitotic counting was performed for a total of $2 \mathrm{~mm}^{2}$ for each case (microscopes used: Zeiss Axioskop 2 plus, eyepiece PI 10/20, fields counted: 10; Leica DM4000 B LED, eyepiece PI 10/20, fields counted: 10; Leica DM2000, eyepiece PI 10/22, fields counted: 8,4). Ki-67 PI was assessed by the experienced pathologists (LH and JvdT) in analogy to routine practice (average counts). ${ }^{25}$ For $\mathrm{MI}$ and $\mathrm{Ki}-67 \mathrm{PI}$ the average value of the two pathologists was used. Cases with a clear diagnosis of SCLC, LCNEC or carcinoid upon pathological review, were excluded from the study.

\section{Immunohistochemistry}

Additional IHC was performed for all selected cases. IHC staining for pRb (13A10, Leica Biosystems) and p53 (DO-7, IR616 DAKO) was performed as described earlier. ${ }^{17,26}$ Staining was assessed by three investigators $(\mathrm{LH}, \mathrm{BH}, \mathrm{CH})$. Nuclear staining for $\mathrm{pRb}$ was scored negative (complete loss or very limited $\mathrm{pRb}$ expression) or positive (preserved pRb expression). ${ }^{17}$ Total loss of p53 expression and strong nuclear overexpression of p53 were considered to be associated with a TP53 mutation. All other cases were considered to be associated with wild type TP53 and scored as 'normal' staining. ${ }^{22}$ 


\section{Statistics}

Data-analysis was conducted with SPSS statistics (version 25 for Windows, Armonk, NY: IBM Corp.). Descriptive statistics were used to present patient characteristics. Median OS was evaluated by Kaplan Meier analysis and presented with $95 \%$ confidence interval (CI) for the total group of stage IV patients and for stage IV patients with well differentiated morphology and preserved $\mathrm{pRb}$ expression.

\section{Results}

\section{Patient selection}

We identified 139 patients, diagnosed and/or treated with pulmonary NEN (non SCLC) at MUMC+ between 2007 and 2018. Based on presence of both carcinoid and NEC characteristics in the pathology reports of these patients, 19 cases were selected (Supplemental Figure S5.A). During pathological review all samples exhibited neuroendocrine morphology and IHC expression for $\geq 2$ neuroendocrine markers (chromogranin A, synaptophysin and/or Cd56). Eleven cases were excluded because a consensus diagnosis of carcinoid $(N=2)$, LCNEC $(N=8)$ or SCLC $(N=1)$ was made, thereby not fulfilling our inclusion criteria. One case was excluded because only very limited material was available (Figure 5.1 \& 5.2, Supplemental Figure S5.A).

\section{Patient characteristics}

Four out of 7 patients were male and median age was 59 years (range 42-82 years). Additional patient characteristics are provided in Table 5.1. All patients had stage IV disease and were treated with palliative systemic therapy.

\section{Tumor characteristics}

Initial diagnosis was LCNEC in $2 / 7$ patients, AC in $2 / 7$ patients, NSCLC with neuroendocrine features in $2 / 7$ patients and the last one had an uncertain diagnosis in between NET and NEC (Table 5.1). A well differentiated morphology was seen in $5 / 7$ cases and a morphology with both well differentiated and poorly differentiated characteristics (equivocal) in 2/7 cases. When applying the WHO 2015 classification after review, 5/7 tumors were classified as LCNEC and 2/7 as carcinoid, based on MI and assessment of necrosis (Table 5.1 \& 5.2). The carcinoid-classified patients were included because of a high $\mathrm{Ki}-67 \mathrm{PI}$ (33\% and 43\%, respectively). Preserved wild type 
pRb IHC expression was seen in 4/7 cases and a staining pattern associated with wild type p53 was seen in 4/7 cases.

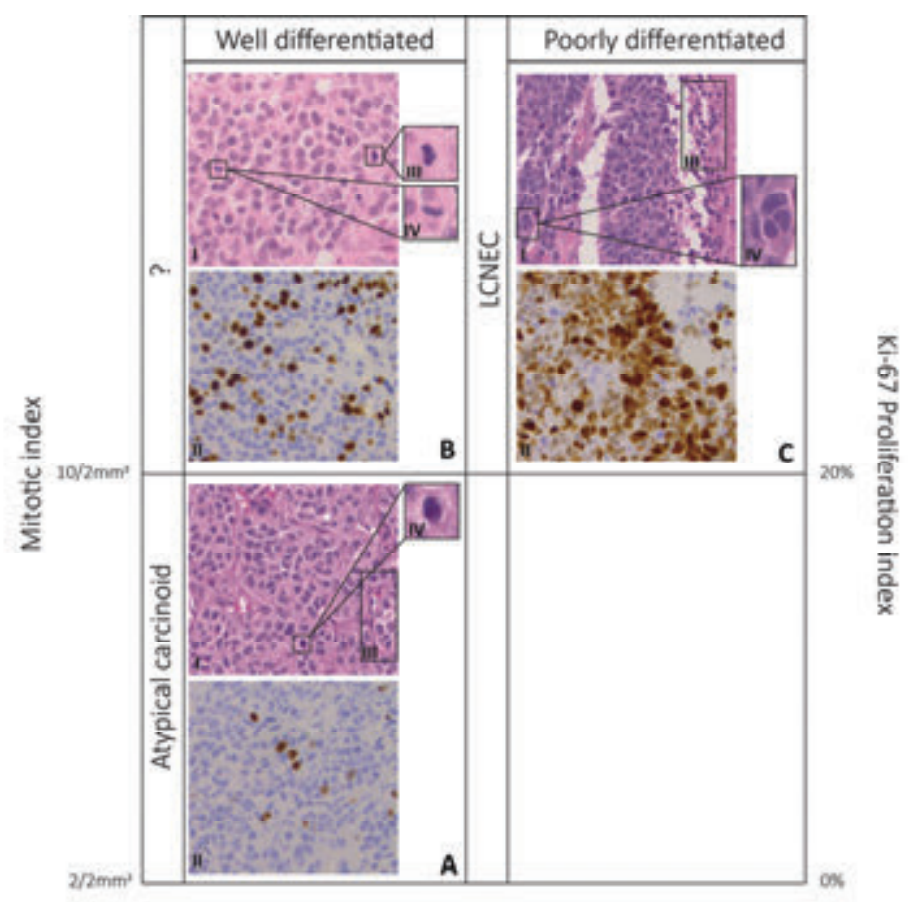

Figure 5.1 Representative cases of HE-staining and Ki-67 immunohistochemistry in atypical carcinoid, large cell neuroendocrine carcinoma and tumors with well differentiated morphology, but high proliferation rate. A) Atypical carcinoid with I) HE-staining (400x), II) Ki-67 (400x), III) rosette-like architecture and tumor cells with round homogenous nuclei, IV) mitosis. B) Well differentiated tumor with high proliferation rate I) HE-staining (400x), II) Ki-67 (400x), III) \& IV) mitosis. C) LCNEC with I) HE-staining (400x), II) Ki-67 (400x), III) necrosis and infiltrating immune cells, IV) angular shaped nuclei with molding.

\section{Survival}

The OS ranged from 2 to 45 months and median OS was 8 months (95\% Cl 5-11 months). The median OS in the group with well differentiated morphology and preserved pRb staining was 45 months ( $95 \% \mathrm{Cl}$ could not be determined). 

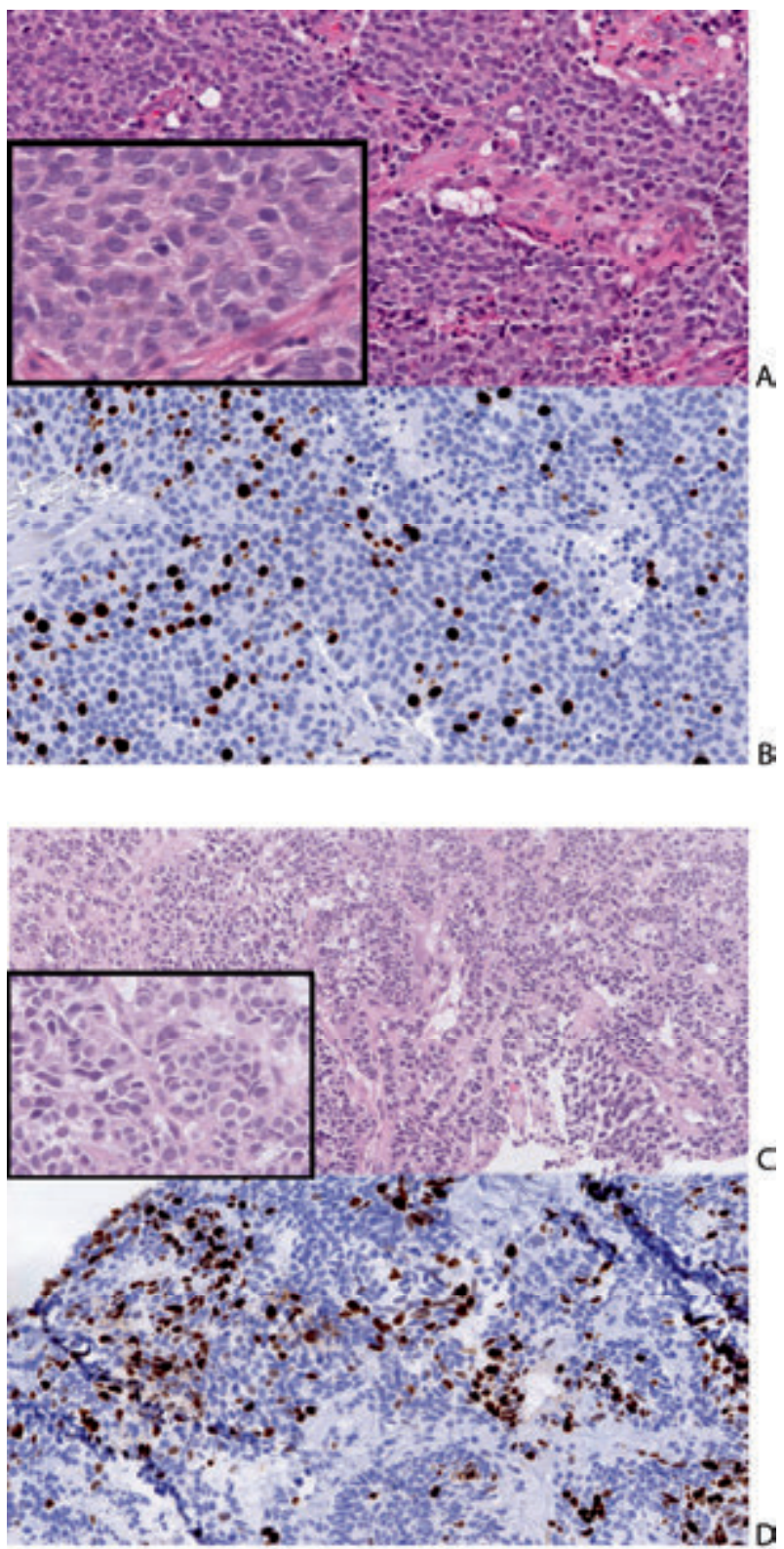

Figure 5.2 Representative cases of tumors with well-differentiated morphology but high mitotic count and/or Ki-67 proliferation index. A) Case C, HE-staining (magnification 200x, box 400x). B) Case C, Ki-67 staining (magnification 200x). C) Case E, HE-staining (magnification 200x, box 400x). D) Case $\mathrm{E}, \mathrm{Ki}-67$ staining (magnification 200x). 


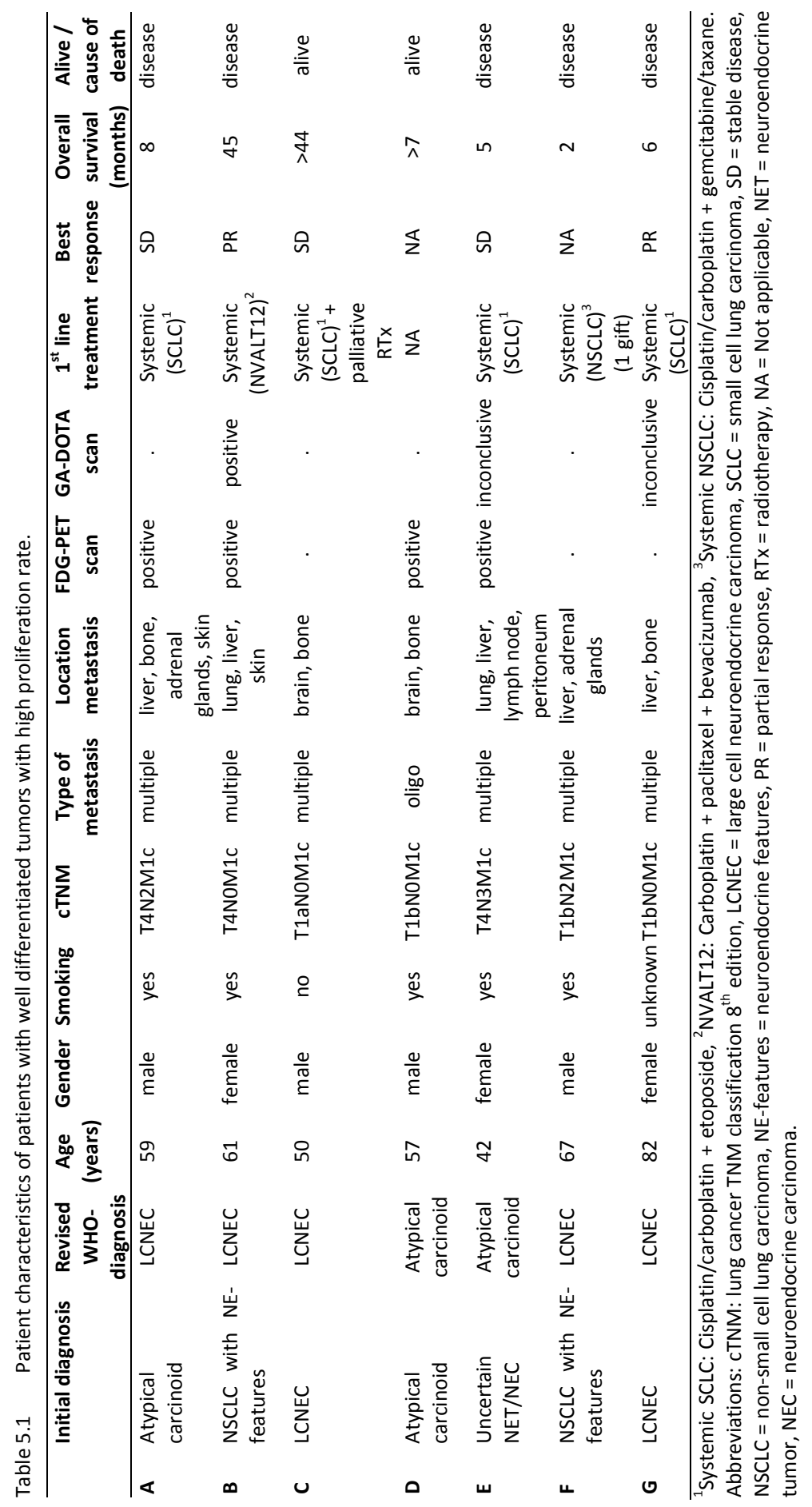




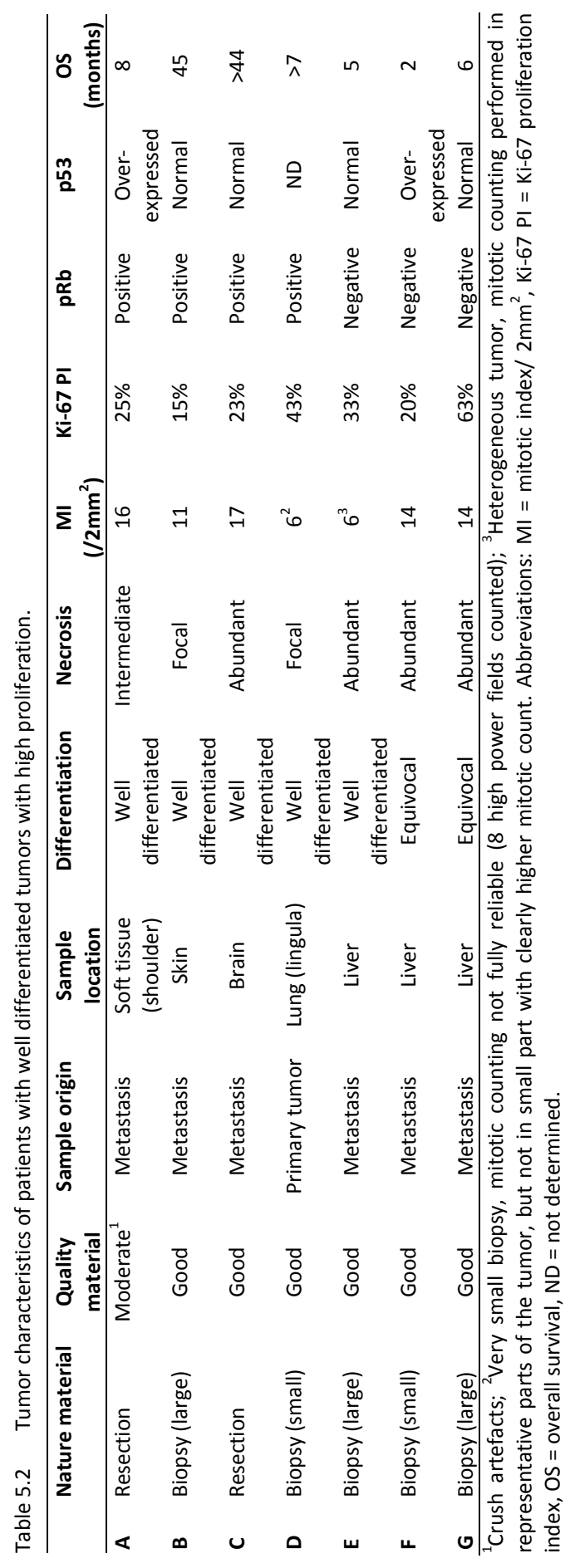




\section{Discussion}

We describe 7 patients with metastatic pulmonary NEN with well differentiated or equivocal morphology and high proliferation rate, most of them currently classified as LCNEC. However, reported OS in stage IV LCNEC is 4-9 months, whereas in this study a longer survival was observed in cases with well differentiated morphology and preserved pRb staining. ${ }^{2,3,6}$

Patients comparable to the cases presented here, with high proliferation rate but well differentiated morphology, have been reported before (Table 5.3). Most of these tumors had well differentiated morphology, but $\mathrm{MI}>10 / 2 \mathrm{~mm}^{2}$, and should therefore be classified as LCNEC according to the WHO criteria. ${ }^{8,10-14,27}$ However, some of the cases had $\mathrm{MI}<10 / 2 \mathrm{~mm}^{2}$ but $\mathrm{Ki}-67 \mathrm{PI}>20 \%$ and should thus be classified as carcinoid according to the current WHO classification. ${ }^{9,13,28}$ Remarkably, one study provided evidence for temporally increased proliferation ( $\geq 10 \%$ increase of $\mathrm{Ki}-67 \mathrm{PI}$ and/or an increase of $\geq 10 / 2 \mathrm{~mm}^{2}$ in $\mathrm{MI}$ ) in $35 \%$ of metastatic lesions from matched primary carcinoid tumor specimens. ${ }^{9}$ Therefore, in analogy to gastrointestinal and pancreatic NEN, cases with (spatially and/or temporally) combined features of both carcinoids and LCNEC may exist.

The significance of well differentiated or equivocal morphology in high-grade pulmonary NEN is unclear. In stage I-III patients described in small case series, a longer OS than expected for LCNEC has been observed in the majority of the patients. ${ }^{8,12,27}$ However, a high frequency of disease recurrence has also been described in those cases. $^{8,13,14,27}$ Furthermore, one study showed a prognosis comparable to LCNEC. ${ }^{28}$ Hence, in this context there might be limited additional value to this subclassification. However, in the metastatic setting, a well differentiated morphology with high-grade proliferation may indicate a more indolent course of disease as shown in this study and in the study by Rehktman et al., although others reported a more heterogenous outcome. $^{9,13}$ Nevertheless, a correlation of improved treatment responses with somatostatin analogues, peptide receptor radionucleotide therapy or everolimus in well differentiated high-grade proliferative pulmonary NENs is currently still lacking. Therefore, clinical impact of this subgroup in the metastatic setting requires further investigation. 


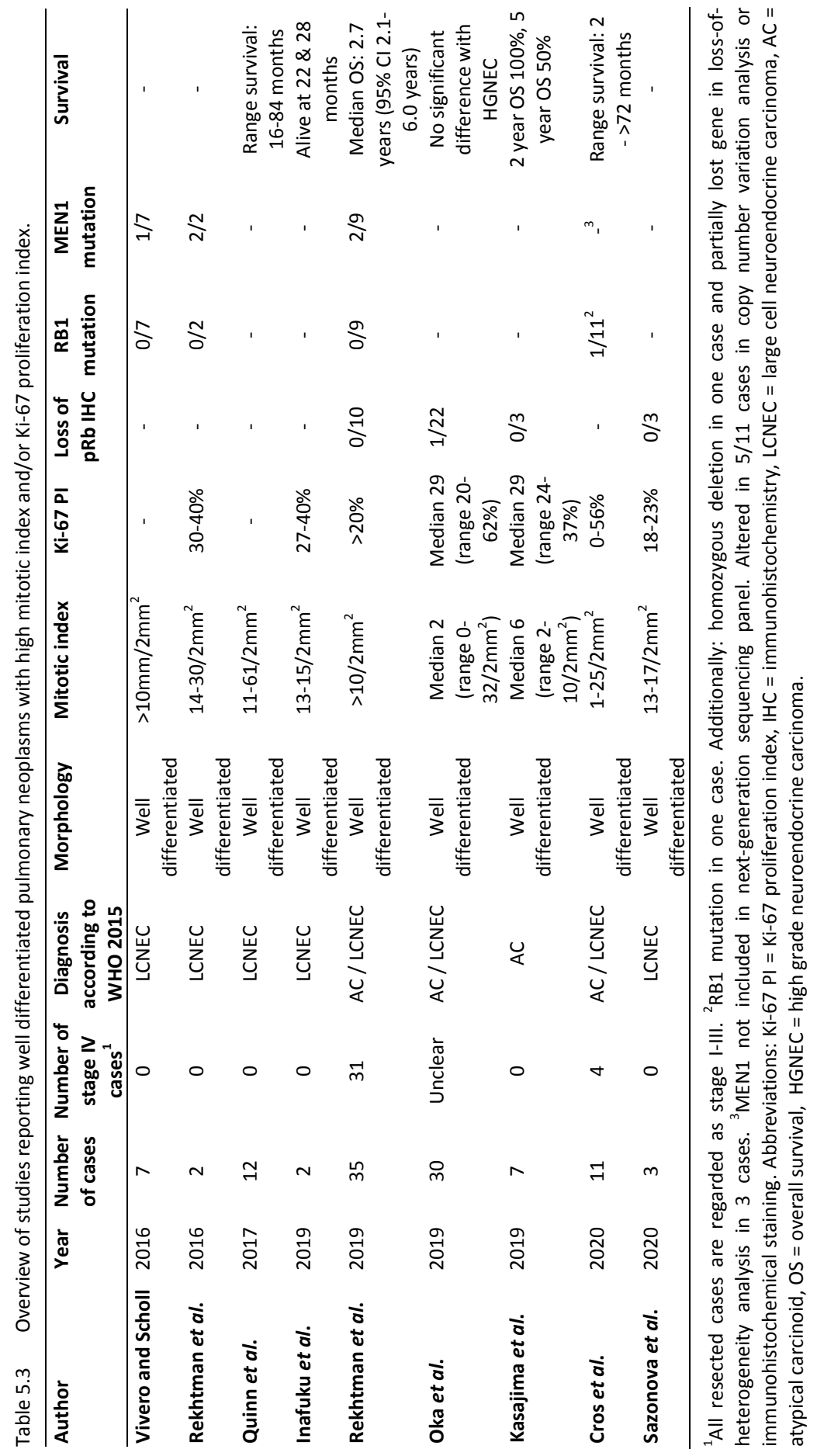


Loss of $\mathrm{pRb}$ expression is frequently seen in LCNEC, but less frequent in carcinoids. ${ }^{17,29-31}$ We noticed preserved $\mathrm{pRb}$ expression in 4 stage IV NEN patients with well differentiated morphology and high proliferation rate and found a relatively long survival (median 45 months) in those patients. In previously reported data of 34 cases, none of the cases showed a $R B 1$ mutation and/or loss of pRb expression. ${ }^{9-11,14,27}$ However, in a recent study alterations in $R B 1$ were found in $5 / 11$ cases. ${ }^{13}$ We found loss of $\mathrm{pRb}$ expression in one case with well differentiated morphology and in two cases with equivocal morphology. Those cases had an overall survival of only 2-6 months. Discrimination between well and poorly differentiated morphology might be challenging with only limited inter-observer concordance and therefore we classified two of the cases in this series as 'equivocal' (characteristics of both well and poorly differentiated morphology). Herewith, we did not intend to add another sub-category, but we intended to highlight the diagnostic challenges of this subgroup of borderline cases. Therefore, based on our results, pRb staining might be useful in these borderline cases as an additional classifying or prognostic marker.

Molecular analyses have also suggested cases on the borderline of AC and LCNEC in addition to histopathological analyses. For example, recently a subgroup of $A C$ with molecular and clinical features more comparable to LCNEC has been identified and named 'supracarcinoids'. ${ }^{19}$ Those supracarcinoids were derived from tumors classified as $\mathrm{AC}$ according to the current WHO classification and had $\mathrm{MI} \leq 4 / 2 \mathrm{~mm}^{2}$ (Ki-67 PI not provided). Therefore, there is an important difference between the cases described by us and others with MI mostly $>10 / 2 \mathrm{~mm}^{2}$, and the supracarcinoids. Another study did evaluate the molecular status of 3 LCNEC cases with well differentiated morphology but lower mitotic count $\left(<20 / 2 \mathrm{~mm}^{2}\right)$ and found that the RNA signature of these tumors was more comparable to AC than to LCNEC. ${ }^{14}$ Finally, an analysis of pure AC and LCNEC tumors indicated a separation into three different molecular cohorts: one enriched for $\mathrm{AC}$, one for LCNEC and one having cases with AC and LCNEC. ${ }^{20}$ Combined these studies suggest that tumors at the borderline of the spectrum AC-LCNEC share both carcinoid and high-grade neuroendocrine carcinoma features requiring additional research enabling separation and that markers such as RB1 and/or MEN1 might enable their differentiation.

It is tempting to speculate about an improved classification of pulmonary NENs. For example, Rindi et al. proposed an alternative grading system in 2014 , based on mitotic count ( $\leq 2 / 10$ high power fields (HPF), >2-47/10HPF, >47/10HPF), Ki-67 PI $(<4 \%, 4-<25 \%$, $\geq 25 \%$ ) and necrosis (absent, $<10 \%,>10 \%$ ). ${ }^{32,33}$ Classification of our cases according to the characteristics proposed by Rindi, resulted in 2 cases being $\mathrm{G} 2$ and 5 cases being $\mathrm{G} 3$ 
(Supplemental Table S5.A). All G2 tumors were pRb positive, whereas only 2/5 G3 cases were $\mathrm{pRb}$ positive. Remarkably, G2 tumors showed high survival rates. This indicates that the classification according to Rindi might be helpful in classification of pulmonary NEN with well differentiated morphology and high proliferation rate. A further advantage of this classification would be that assessing a tumor to be well or poorly differentiated is not necessary. However, assessing the amount of necrosis and mitotic counting can also be subjective. ${ }^{34}$ In any case, external validation with a large set of pulmonary NENs is required before adaptation of classification systems may be considered.

The present study has some limitations. First, this was a single center study and due to low incidence of pulmonary NENs in general and especially of NENs with well differentiated morphology but high proliferation rate, we have only identified 7 patients so far. For some patients in this study, only a limited amount of material was available for pathological review. A small biopsy might not reflect behavior of the total tumor in case of heterogeneity and material from metastatic lesions might be slightly different from the primary tumor. However, because we used available clinical material, our study is a good reflection of daily clinical practice. Finally, we used $\mathrm{pRb}$ and p53 immunostainings as surrogate markers for RB1 and TP53 gene mutational status. For $R B 1$, it is known that $\mathrm{pRb}$ provides a good reflection of mutational status and might even be more relevant, since loss of functional $\mathrm{pRb}$ have been observed in cases with wild type $R B 1 .{ }^{17}$ However, concordance between TP53 mutations and p53 staining patterns is less clear. Although total loss of p53 and strong nuclear overexpression are associated with TP53 mutations, tumors with 'normal' wild type IHC expression might still harbor a mutation and sometimes intense and diffuse IHC staining is seen as a physiological reaction to cell damage. ${ }^{22}$ This might be the reason that no correlation between $\mathrm{p} 53$ staining and survival has been found in this study.

In conclusion, we have reported 7 stage IV pulmonary NEN cases with well differentiated or equivocal morphology but high proliferation rate. Cases with well differentiated morphology and preserved $\mathrm{pRb}$ expression had a relatively high median survival. Future studies should evaluate the clinical value of $\mathrm{pRb}$ as a prognostic marker in patients with metastatic disease. 


\section{References}

1. Rindi G, Klimstra DS, Abedi-Ardekani B, et al. A common classification framework for neuroendocrine neoplasms: an International Agency for Research on Cancer (IARC) and World Health Organization (WHO) expert consensus proposal. Mod Pathol 2018;31:1770-1786.

2. Travis WD, Brambilla E, Burke AP, et al. WHO Classification of Tumours of the Lung, Pleura, Thymus and Heart Lyon: International Agency for Research on Cancer, 4th edition, March 2015; 2015.

3. Derks JL, Hendriks LE, Buikhuisen WA, et al. Clinical features of large cell neuroendocrine carcinoma: a population-based overview. Eur Respir J 2016;47:615-624.

4. He $\mathrm{Y}$, Liu $\mathrm{H}$, Wang $\mathrm{S}$, et al. Prognostic nomogram predicts overall survival in pulmonary large cell neuroendocrine carcinoma. PloS one 2019;14:e0223275.

5. Asamura H, Kameya T, Matsuno Y, et al. Neuroendocrine neoplasms of the lung: a prognostic spectrum. J Clin Oncol 2006;24:70-76.

6. Kinslow CJ, May MS, Saqi A, et al. Large-Cell Neuroendocrine Carcinoma of the Lung: A PopulationBased Study. Clin Lung Cancer 2020;21(2):e99-e113.

7. Sullivan I, Le Teuff G, Guigay J, et al. Antitumour activity of somatostatin analogues in sporadic, progressive, metastatic pulmonary carcinoids. Eur J Cancer 2017;75:259-267.

8. Quinn AM, Chaturvedi A, Nonaka D. High-grade Neuroendocrine Carcinoma of the Lung With Carcinoid Morphology: A Study of 12 Cases. Am J Surg Pathol 2017;41:263-270.

9. Rekhtman N, Desmeules P, Litvak AM, et al. Stage IV lung carcinoids: spectrum and evolution of proliferation rate, focusing on variants with elevated proliferation indices. Mod Pathol 2019;32: 1106-1122.

10. Rekhtman N, Pietanza MC, Hellmann MD, et al. Next-Generation Sequencing of Pulmonary Large Cell Neuroendocrine Carcinoma Reveals Small Cell Carcinoma-like and Non-Small Cell Carcinoma-like Subsets. Clin Cancer Res 2016;22:3618-3629.

11. Vivero M, Scholl LM. "Borderline" neuroendocrine carcinomas of the lung are clinically and genomically distinct from large cell neuroendocrine carcinoma. Mod Pathol 2016;29.

12. Inafuku $\mathrm{K}$, Yokose $\mathrm{T}$, Ito $\mathrm{H}$, et al. Two cases of lung neuroendocrine carcinoma with carcinoid morphology. Diagn Pathol 2019;14:104.

13. Cros J, Theou-Anton N, Gounant V, et al. Specific genomic alterations in high grade pulmonary neuroendocrine tumours with carcinoid morphology. Neuroendocrinology 2020.

14. Sazonova O, Manem V, Orain M, et al. Transcriptomic data helps refining classification of pulmonary carcinoid tumors with increased mitotic counts. Mod Pathol 2020;33(9):1712-1721.

15. WHO. WHO classification of Tumours, Digestive System Tumours, 5th edition. WHO; 2019.

16. Lloyd RV, Osamura RY, Kloppel G, et al. WHO classification of Tumours of Endocrine Organs. Lyon: International Agency for Research on Cancer; 2017.

17. Derks JL, Leblay N, Thunnissen E, et al. Molecular Subtypes of Pulmonary Large-cell Neuroendocrine Carcinoma Predict Chemotherapy Treatment Outcome. Clin Cancer Res 2018;24:33-42.

18. George J, Walter V, Peifer M, et al. Integrative genomic profiling of large-cell neuroendocrine carcinomas reveals distinct subtypes of high-grade neuroendocrine lung tumors. Nat Commun 2018;9:1048.

19. Alcala N, Leblay N, Gabriel AAG, et al. Integrative and comparative genomic analyses identify clinically relevant pulmonary carcinoid groups and unveil the supra-carcinoids. Nat Commun 2019;10:3407.

20. Simbolo M, Barbi S, Fassan M, et al. Gene Expression Profiling of Lung Atypical Carcinoids and Large Cell Neuroendocrine Carcinomas Identifies Three Transcriptomic Subtypes with Specific Genomic Alterations. J Thorac Oncol 2019;14:1651-1661.

21. Hashimoto $T$, Tokuchi $\mathrm{Y}$, Hayashi $\mathrm{M}$, et al. p53 null mutations undetected by immunohistochemical staining predict a poor outcome with early-stage non-small cell lung carcinomas. Cancer Res 1999;59:5572-5577.

22. Yemelyanova A, Vang R, Kshirsagar M, et al. Immunohistochemical staining patterns of p53 can serve as a surrogate marker for TP53 mutations in ovarian carcinoma: an immunohistochemical and nucleotide sequencing analysis. Mod Pathol 2011;24:1248-1253. 
23. Kobel M, Ronnett BM, Singh N, et al. Interpretation of P53 Immunohistochemistry in Endometrial Carcinomas: Toward Increased Reproducibility. Int J Gynecol Pathol 2019;38 Suppl 1:S123-S131.

24. Achcar RD, Groshong SD, Cool CD. Pulmonary Pathology. Philadeplhia: Wolters Kluwer Health; 2017.

25. Warth A, Fink L, Fisseler-Eckhoff A, et al. Interobserver agreement of proliferation index (Ki-67) outperforms mitotic count in pulmonary carcinoids. Virchows Arch 2013;462:507-513.

26. Pierssens D, Borgemeester MC, van der Heijden SJH, et al. Chromosome instability in tumor resection margins of primary OSCC is a predictor of local recurrence. Oral Oncol 2017;66:14-21.

27. Kasajima A, Konukiewitz B, Oka N, et al. Clinicopathological Profiling of Lung Carcinoids with a Ki67 Index > 20. Neuroendocrinology 2019;108:109-120.

28. Oka N, Kasajima A, Konukiewitz B, et al. Classification and prognostic stratification of bronchopulmonary neuroendocrine neoplasms. Neuroendocrinology 2019.

29. Cagle PT, el-Naggar AK, Xu HJ, et al. Differential retinoblastoma protein expression in neuroendocrine tumors of the lung. Potential diagnostic implications. The American journal of pathology 1997;150: 393-400.

30. Gugger M, Burckhardt E, Kappeler A, et al. Quantitative expansion of structural genomic alterations in the spectrum of neuroendocrine lung carcinomas. J Pathol 2002;196:408-415.

31. Beasley MB, Lantuejoul S, Abbondanzo S, et al. The P16/cyclin D1/Rb pathway in neuroendocrine tumors of the lung. Hum Pathol 2003;34:136-142.

32. Rindi G, Klersy C, Inzani F, et al. Grading the neuroendocrine tumors of the lung: an evidence-based proposal. Endocr Relat Cancer 2014;21:1-16.

33. Swarts DR, Rudelius M, Claessen SM, et al. Limited additive value of the Ki-67 proliferative index on patient survival in World Health Organization-classified pulmonary carcinoids. Histopathology 2017;70:412-422.

34. Swarts DR, van Suylen RJ, den Bakker MA, et al. Interobserver variability for the WHO classification of pulmonary carcinoids. Am J Surg Pathol 2014;38:1429-1436. 


\section{Supplemental material}

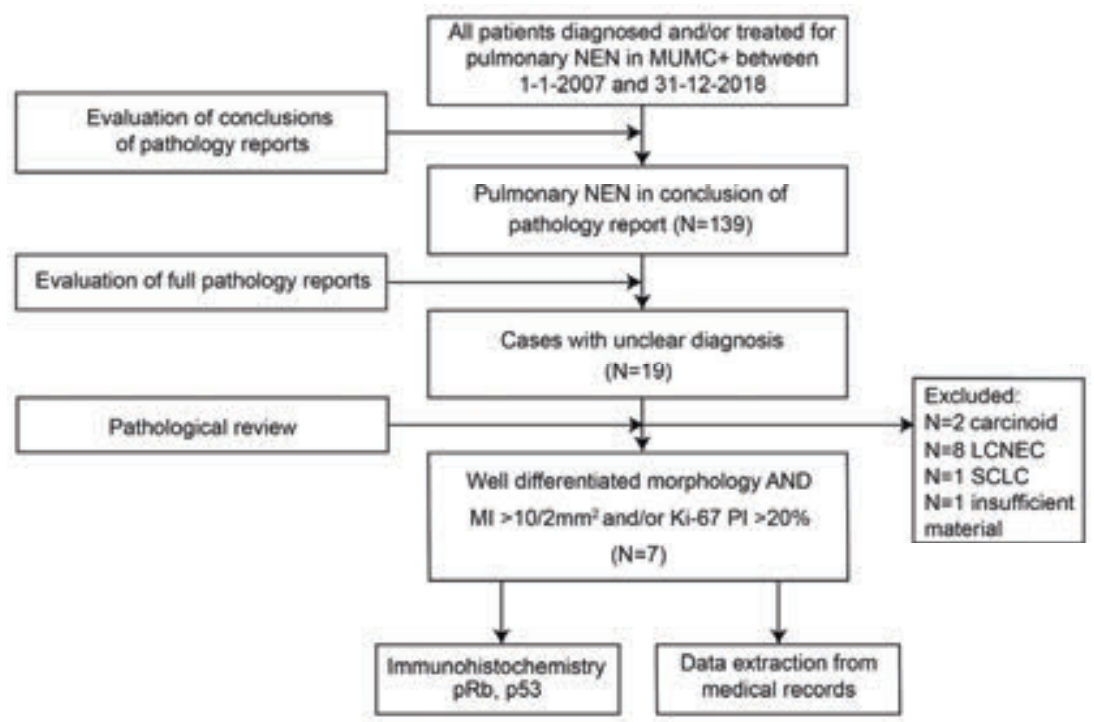

Figure S5.A Selection of cases for pathological review and inclusion in the study. Abbreviations: NEN = neuroendocrine neoplasm, MUMC+ = Maastricht University Medical Center, LCNEC = large cell neuroendocrine carcinoma, $\mathrm{SCLC}=$ small cell lung carcinoma, $\mathrm{MI}=$ mitotic index, $\mathrm{Ki}-67 \mathrm{PI}=\mathrm{Ki}-67$ proliferation index.

Table S5.A Correlation between overall survival, classification according to Rindi* and pRb IHC status in pulmonary NEN with well differentiated morphology and high proliferation rate.

\begin{tabular}{cccc}
\hline & Overall survival (months) & Rindi-classification & IHC $\mathbf{p R b}$ \\
\hline $\mathrm{B}$ & 45 & $\mathrm{G} 2$ & Positive \\
$\mathrm{C}$ & $>44$ & $\mathrm{G} 3$ & Positive \\
$\mathrm{D}$ & $>7$ & $\mathrm{G} 2$ & Positive \\
$\mathrm{A}$ & 8 & $\mathrm{G} 3$ & Positive \\
$\mathrm{G}$ & 6 & $\mathrm{G} 3$ & Negative \\
$\mathrm{E}$ & 5 & $\mathrm{G} 3$ & Negative \\
$\mathrm{F}$ & 2 & $\mathrm{G} 3$ & Negative \\
\hline
\end{tabular}

*Rindi et al., Endocr Relat Cancer, 2014. Abbreviations: NEN = neuroendocrine neoplasms, IHC = immunohistochemistry, ND = not determined. 



\section{Chapter 6}

\section{Delta like ligand 3 (DII3) as target for treatment of small cell lung carcinoma (SCLC) and pulmonary large cell neuroendocrine carcinoma (LCNEC)}

B.C.M. Hermans, J.L. Derks, L.E.L. Hendriks, L. Moonen, E.J.M. Speel, A-M.C. Dingemans With permission adapted from: Ned Tijdschr Oncol 2019;16:325-33 


\section{Abstract}

Pulmonary neuroendocrine carcinoma can be divided in small cell lung carcinoma (SCLC, around $15 \%$ of all lung tumors) and large cell neuroendocrine carcinoma (LCNEC, around $1-3 \%$ of all lung tumors). In contrast to non-small cell lung carcinoma, no targeted therapy is yet approved for SCLC and LCNEC. However, there is an urgent need for more effective therapy to improve survival rates in patients with these tumors. Delta like ligand 3 (DII3) is expressed in $64-90 \%$ of SCLC and LCNEC, whereas no or only very limited expression is observed in normal tissues. Therefore, DIl3 might be an interesting target for therapy. Currently, three different approaches using AntibodyDrug Conjugates (ADC), Bispecific T-cell Engaging antibodies (BiTE $\left.{ }^{\circledR}\right)$ and Chimeric Antigen Receptor T-cells (CAR-T), are in development for Dll3 targeted therapy. Preclinical studies and a phase 1 study with the ADC rovalpituzumab-tesirine (Rova-T) showed successful internalization of the toxin in Dll3 positive tumor cells and a sustained response. A phase 2 study (TRINITY) was less promising. Enrollment of two phase 3 studies (TAHOE and MERU) was ceased after interim analysis by the independent data monitoring committee and development of Rova-T was halted. Drugs using $\mathrm{BiTE}^{\circledR}$ and CAR-T approaches are in development and phase 1 trials are recruiting. Although DIl3 is a potential target, efficacy of those DII3 targeted drugs has to be demonstrated. 


\section{Introduction}

High grade pulmonary neuroendocrine carcinoma can be subdivided in small cell lung carcinoma (SCLC, $15 \%$ of all lung cancer) and large cell neuroendocrine carcinoma (LCNEC, 1-3\% of all lung cancer). The majority of SCLC and LCNEC patients present with metastatic disease. Both SCLC and LCNEC have an unfavorable prognosis, with a median overall survival (OS) $<10$ months for stage IV disease. ${ }^{1,2}$ For metastatic SCLC, treatment of choice is palliative chemotherapy with cisplatin/carboplatin and etoposide. ${ }^{3}$ Stage IV LCNEC is also treated with palliative intent, using platinum-based chemotherapy in combination with etoposide (SCLC-protocol), or in combination with gemcitabine or taxane (non-small cell lung carcinoma (NSCLC)-protocol). ${ }^{4,5}$ For NSCLC, the introduction of targeted therapy and immunotherapy has resulted in major survival benefits for a selected group of stage IV patients. Recently, atezolizumab has been registered as immunotherapy in SCLC, in combination with platinum containing chemotherapy and etoposide. However, the survival benefit is limited. ${ }^{6}$ Due to the poor prognosis of SCLC and LCNEC, there is a high demand for new therapeutic options. However, no other new treatments has been registered as a substitution for or as a supplement to palliative chemotherapy in the last decades.

Delta like ligand 3 (DII3) is a promising target for therapy in pulmonary neuroendocrine carcinoma. ${ }^{7,8}$ In this paper we discuss the current evidence for treatment of SCLC and LCNEC with DII3 targeted therapy.

\section{Notch ligand DII3}

The Notch-pathway is an intracellular signalling mechanism including 4 Notch receptors (Notch1-4). These Notch receptors interact with transmembrane ligands coded by jagged (JAG1 and JAG2) and delta-like (DLL1, DLL3 and DLL4) gene families. DII1 and DII4 have a stimulatory effect after binding to a Notch receptor of another cell (transactivation). However, expression of the ligand in the same cell as the receptor results in an inhibitory effect (cis-inhibition). ${ }^{9}$ In contrast, the exclusive function of DII3 seems to be Notch1 inhibition. ${ }^{10-12}$ DII3 itself is regulated by achaete-scute complex-like 1 (Ascl1), a transcription factor involved in development of pulmonary neuroendocrine cells. ${ }^{13,4}$ High expression of Ascl1 will result in more Dll3 expression and inhibition of Notch1. Complementary, overexpression of Notch1 has been shown to inhibit Ascl1..$^{15,16}$ Apparently, the Ascl1-DIl3-Notch1 pathway is a non-linear pathway where all proteins influence each other. Both Notch1 inhibition (mutation or downregulation) and ascl1 
activation have an important role in development of neuroendocrine neoplasms. ${ }^{17,18}$ This is in contrast with oncogenic activation of Notch in other solid tumors. ${ }^{19,20}$

\section{Dll3 expression in neuroendocrine carcinoma}

Several studies have described DII3 expression in $64-90 \%$ of SCLC and LCNEC, using protein immunohistochemistry (IHC) or mRNA reverse transcriptase polymerase chain reaction (PCR) (Table 6.1)..$^{7,8,21-27}$ The Dll3 antibodies SC16.65 (Abbvie) and SP347 (Ventana) do not seem to exhibit important differences, whereas expression levels of ab103102 (Abcam) seem to be slightly higher. ${ }^{27}$ High Dll3 expression was found in $32-83 \%$ of tumors ( $\mathrm{H}$-score $>100$ or percentage of positive tumor cells $\geq 50 \%$ or $\geq 75 \%$, respectively) (Table 6.1). ${ }^{7,8,21,22,25-29} \mathrm{~A}{ }^{89} \mathrm{Zr}$-labeled DII3 antibody has been developed as a predictive marker, allowing the evaluation of DII3 binding to the tumor with a positron-emission tomography (PET)-scan. With this method, DII3 expression and treatment response could be predicted in patient derived xenograft (PDX)-models. ${ }^{30}$ So far, no prognostic effect of DII3 expression has been shown in SCLC or LCNEC patients. $^{21,25,26,31}$

Table 6.1 DII3 expression in SCLC and LCNEC.

\begin{tabular}{|c|c|c|c|c|c|c|c|}
\hline References & Year & Tumor type (N) & $\begin{array}{l}\text { DII3 IHC } \\
\text { antibody } \\
\text { clone or } \\
\text { RT-PCR } \\
\text { probe }\end{array}$ & $\begin{array}{c}\text { Cut-off value } \\
\text { DII3 }\end{array}$ & $\begin{array}{c}\text { Dll3 } \\
\text { expression }\end{array}$ & $\begin{array}{l}\text { High DII3 } \\
\text { expression }\end{array}$ & $\begin{array}{c}\text { Survival (DII3+ } \\
\text { vs. Dll3-) }\end{array}$ \\
\hline \multicolumn{8}{|l|}{ Immunohistochemistry } \\
\hline Saunders et al. ${ }^{8}$ & & LCNEC (57) & SC16.65 & $\mathrm{H}$-score $>100$ & NR & $37(65 \%)^{*}$ & NR \\
\hline Rudin et $a l^{7}$ & 2017 & $\mathrm{SCLC}+\mathrm{LCNEC}(48)$ & SC16.65 & $\geq 1 \%$ & $42(88 \%)$ & $32(67 \%)^{* *}$ & $N R$ \\
\hline Tanaka et al. ${ }^{21}$ & 2018 & $\operatorname{SCLC}(63)$ & SC16.65 & $\geq 1 \%$ & $52(83 \%)$ & $20(32 \%)^{* *}$ & No difference \\
\hline Hermans et al. ${ }^{26}$ & 2019 & LCNEC (94) & SC16.65 & $\geq 1 \%$ & $70(74 \%)$ & $51(54 \%)^{* *}$ & No difference \\
\hline Saito et $a .^{22}$ & 2018 & $\operatorname{SCLC~(20)~}$ & ab103102 & $\geq 1 \%$ & $18(90 \%)$ & $14(70 \%)^{* *}$ & NR \\
\hline Brcic et al. ${ }^{27}$ & & LCNEC (27) & SP347 & $\geq 25 \%$ & $14(52 \%)$ & NR & NR \\
\hline Xie et $a l^{28}$ & 2019 & SCLC (44) & SP347 & $\geq 50 \%$ & NR & $35(83 \%)^{* *}$ & No difference \\
\hline Messaritakis et al. ${ }^{29}$ & 2019 & $\operatorname{SCLC~(20)~}$ & SP347 & $\geq 50 \%$ & NR & $14(70 \%)^{* *}$ & NR \\
\hline Furuta et $a l^{25}$ & 2019 & SCLC (93) & NR & $\geq 1 \%$ & 77 (83\%) & $44(47 \%)^{* * *}$ & No difference \\
\hline \multicolumn{8}{|l|}{ RT-PCR } \\
\hline Roy et $a .^{24}$ & $\begin{array}{c}2017 \\
\text { (abstract) }\end{array}$ & $\operatorname{SCLC~(58)~}$ & $\begin{array}{c}\text { Probe } \\
\text { unknown }\end{array}$ & NR & 37 (64\%) & NR & NR \\
\hline
\end{tabular}

*Samples with DII3 H-score >100; **Samples with Dll3 expression in $\geq 50 \%$ tumor cells; ${ }^{* * *}$ Samples with DII3 expression in $\geq 75 \%$ tumor cells. Abbreviations: IHC = immunohistochemistry, SCLC = small cell lung carcinoma, NR = not reported, LCNEC = large cell neuroendocrine carcinoma (pulmonary). 
DII3 expression has also been demonstrated in other tumors than LCNEC and SCLC. The expression of DII3 in neuroendocrine carcinoma of the prostate, bladder and skin (Merkel cell carcinoma) is comparable to SCLC and LCNEC (Table 6.2). ${ }^{31-34}$ Furthermore, high expression has been shown in melanoma, testicular carcinoma, medullar thyroid cancer, glioma, and glioblastoma (55-90\%). ${ }^{33,35}$ Surprisingly, Dll3 expression (43-80\%) was also found in two studies of NSCLC, both with and without neuroendocrine features. ${ }^{33,36}$ In contrast, only low percentages of positive samples (9-37\%) were found in neuroendocrine carcinoma of the pancreas and breast, low-grade pulmonary neuroendocrine tumors (carcinoids) and several non-neuroendocrine carcinomas (including cerebrum, liver and rectum) (Table 6.2). ${ }^{23,28,33,37}$ A prognostic effect of DII3 expression has been shown in prostate cancer, melanoma and neuroendocrine bladder cancer. $^{28,32-34}$

In contrast to high expression in neuroendocrine carcinoma, DII3 expression is almost absent in normal tissue. Only limited DII3 mRNA expression has been shown in cerebral, esophageal and pancreatic tissue. ${ }^{8}$ Furthermore, Huang et al. found low DII3 IHC expression in adrenal glands, cerebrum, pancreas, pituitary gland, testes, thyroid, stomach, liver, and larynx, but not in other tissue. Remarkably, a neural or neuroendocrine component was present in almost all tissues with DII3 expression. ${ }^{23}$

Clinical relevant cut-off values and relevance of various staining patterns has not been clarified yet. In general, a tumor is regarded positive if any DII3 expression is seen in $\geq 1 \%$ of tumor cells. Furthermore, a subgroup with high Dll3 expression is defined for tumors with $\geq 50 \%$ or $\geq 75 \%$ Dll3 positive tumor cells (Table $6.1,6.2$ ). Three staining patterns has been described for DII3. Frequently, a cytoplasmic staining pattern is seen, which seems to be accompanied by membranous staining (Figure 6.1). Moreover, a punctated staining pattern is observed (Figure 6.1). Furthermore, solely membranous staining is seen infrequently. ${ }^{8,26}$ 
Table 6.2 Dll3 expression in other neoplasms (non SCLC, non LCNEC).

\begin{tabular}{|c|c|c|c|c|c|c|c|}
\hline References & Year & Tumor type (N) & $\begin{array}{c}\text { DIl3 IHC } \\
\text { antibody } \\
\text { clone or RT- } \\
\text { PCR probe }\end{array}$ & $\begin{array}{c}\text { Cut-off } \\
\text { value } \\
\text { Dll3 }\end{array}$ & $\begin{array}{c}\text { DII3 } \\
\text { expression }\end{array}$ & $\begin{array}{l}\text { High DII3 } \\
\text { expression }\end{array}$ & $\begin{array}{c}\text { Survival (DII3+ } \\
\text { vs. DII3-) }\end{array}$ \\
\hline \multicolumn{8}{|c|}{ Immunohistochemistry } \\
\hline \multirow[t]{10}{*}{$\begin{array}{l}\text { Saunders et } \\
\text { al. }^{33}\end{array}$} & $\begin{array}{l}2017 \\
\text { (Abstract) }\end{array}$ & Melanoma* & SC16.65 & NR & $55 \%$ & NR & $\begin{array}{l}\text { Lower OS in } \\
\text { Dll3+ }\end{array}$ \\
\hline & & Low-grade glioma* & & & $90 \%$ & NR & No difference \\
\hline & & Glioblastoma* & & & $70 \%$ & NR & No difference \\
\hline & & $\begin{array}{l}\text { Medullar thyroid } \\
\text { carcinoma* }\end{array}$ & & & $65 \%$ & NR & No difference \\
\hline & & Carcinoids* & & & $33 \%$ & NR & No difference \\
\hline & & NET pancreas* & & & $9 \%$ & NR & No difference \\
\hline & & NET bladder* & & & $57 \%$ & NR & $\begin{array}{l}\text { Lower OS in } \\
\text { Dll3+ }\end{array}$ \\
\hline & & NET prostate* & & & $24 \%$ & NR & No difference \\
\hline & & $\begin{array}{l}\text { Testicular } \\
\text { carcinoma* }\end{array}$ & & & $90 \%$ & NR & No difference \\
\hline & & $\begin{array}{l}\text { NSCLC with } \\
\text { neuroendocrine } \\
\text { features* }\end{array}$ & & & $80 \%$ & NR & No difference \\
\hline Spino et $a l^{35}$ & 2018 & Glioma (46) & SC16.65 & $\geq 1 \%$ & $36(78 \%)$ & $\begin{array}{c}19 \\
(41 \%)^{* *}\end{array}$ & NR \\
\hline $\begin{array}{l}\text { Koshkin et } \\
a .^{32}\end{array}$ & 2018 & $\begin{array}{l}\text { Small-cell bladder } \\
\text { carcinoma (53) }\end{array}$ & SC16.65 & $\geq 1 \%$ & $36(68 \%)$ & $\mathrm{NR}$ & $\begin{array}{l}\text { Lower OS in } \\
\text { Dll3+ }\end{array}$ \\
\hline Xie et $a l^{31}$ & $\begin{array}{l}2019 \\
\text { (Abstract) }\end{array}$ & $\begin{array}{l}\text { Merkel cell } \\
\text { carcinoma (65) }\end{array}$ & SC16.65 & $\geq 1 \%$ & $58(89 \%)$ & $\begin{array}{c}24 \\
(52 \%)^{* *}\end{array}$ & No difference \\
\hline Vranic et al. ${ }^{37}$ & 2019 & NEC breast (19) & SP347 & $\geq 1 \%$ & $2(11 \%)$ & $0(0 \%)^{* *}$ & NR \\
\hline Huang et $a l^{23}$ & ${ }^{3} 2019$ & $\begin{array}{l}\text { Non } \\
\text { neuroendocrine } \\
\text { carcinoma (65) }\end{array}$ & SP347 & $\geq 1 \%$ & $25 \%$ & NR & NR \\
\hline \multirow[t]{2}{*}{ Puca et al. ${ }^{34}$} & 2019 & $\begin{array}{l}\text { Castration resistant } \\
\text { prostate NEC (47) }\end{array}$ & SP347 & $\geq 1 \%$ & $36(77 \%)$ & NR & $\begin{array}{l}\text { Lower OS in } \\
\text { Dll3+ }\end{array}$ \\
\hline & & $\begin{array}{l}\text { Castration resistant } \\
\text { prostate } \\
\text { adenocarcinoma } \\
\text { (56) }\end{array}$ & & & $7(13 \%)$ & NR & $\begin{array}{l}\text { Lower OS in } \\
\text { Dll3+ }\end{array}$ \\
\hline \multirow[t]{2}{*}{ Xie et $a l^{28}$} & 2019 & $\begin{array}{l}\text { Typical carcinoid } \\
\text { (67) }\end{array}$ & SP347 & $\geq 50 \%$ & NR & $\begin{array}{c}22 \\
(33 \%)^{* *}\end{array}$ & No difference \\
\hline & & $\begin{array}{l}\text { Atypical carcinoid } \\
\text { (46) }\end{array}$ & SP347 & $\geq 50 \%$ & NR & $\begin{array}{c}17 \\
(37 \%)^{* *}\end{array}$ & No difference \\
\hline \multicolumn{8}{|l|}{ RT-PCR } \\
\hline $\begin{array}{l}\text { Pancewicz et } \\
\text { al. }^{36}\end{array}$ & 2017 & NSCLC (61) & $\begin{array}{c}\text { TaqMan } \\
\text { probe } \\
\text { Hs0108509 } \\
6 \_m 1\end{array}$ & NR & $21(43 \%)$ & NR & NR \\
\hline
\end{tabular}

*Number of patients not mentioned in abstract; **Samples with Dll3 expression in $\geq 50 \%$ tumor cells. Abbreviations: SCLC = small cell lung carcinoma, LCNEC = large cell neuroendocrine carcinoma (pulmonary), $\mathrm{IHC}=$ immunohistochemistry, $\mathrm{NR}=$ not reported, $\mathrm{OS}=$ overall survival, $\mathrm{NET}=$ neuroendocrine tumor, $\mathrm{NSCLC}=$ non-small cell lung carcinoma, NEC = neuroendocrine carcinoma, RT-PCR = reverse transcriptase polymerase chain reaction. 

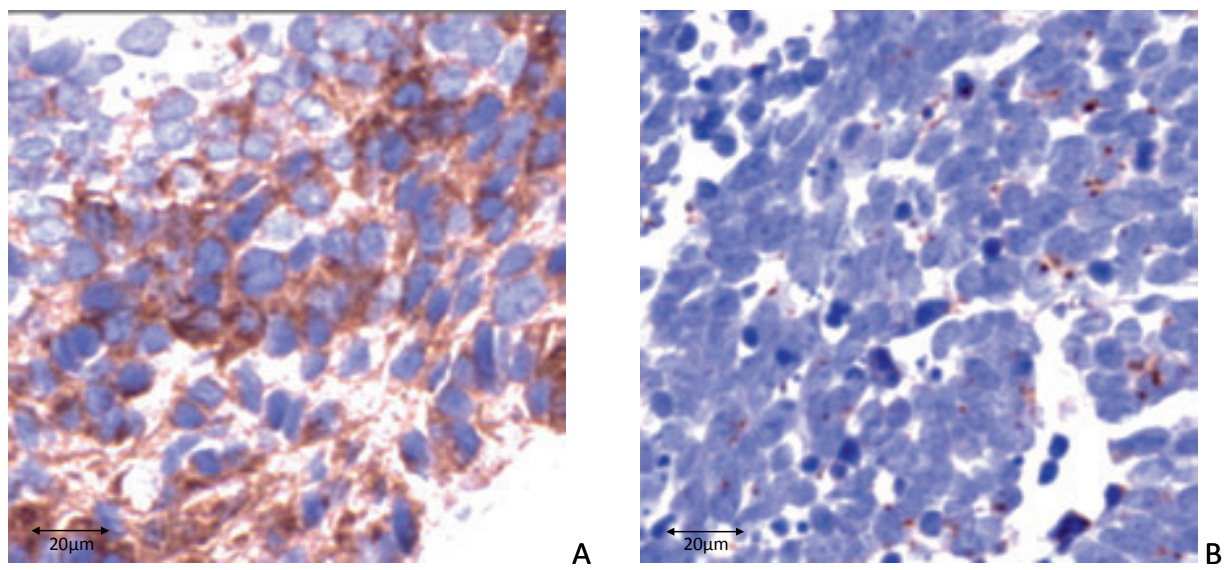

Figure 6.1 Representative LCNEC cases showing different types of immunohistochemical staining patterns of DII3 expression (brown dab). Cell nuclei are counterstained with hematoxylin. A) Combined cytoplasmic and membranous staining. B) Punctated staining.

\section{Targeted DII3 therapy}

\section{Rova-T}

The first-in-class DII3 targeted therapy is the antibody-drug conjugate rovalpituzumabtesirine (Rova-T). ${ }^{38}$ For this drug, a Dll3 specific antibody (rovalpituzumab) has been coupled to a toxin (tesirine). After binding of the antibody to Dll3 on the tumor cell, internalization of the conjugate will take place and the tumor cell will be exposed to a relatively high dose of toxin (Figure $6.2 \mathrm{~A}$ ). ${ }^{38}$ Preclinical studies have shown both internalization and cytotoxicity in DII3 positive cell lines and PDX-models of SCLC, LCNEC, glioma and prostate cancer. ${ }^{8,34,35}$ Saunders et al. found a sustained response (>150 days) after treatment with Rova-T in two PDX-models (SCLC LU64 and LCNEC LU37), whereas only a limited response of <30 days was seen after treatment of the same models with cisplatin-etoposide. ${ }^{8}$ In a third model (SCLC LU86), treatment with Rova-T was also superior to cisplatin-etoposide, however, progression was seen after 30 days. $^{8}$ Furthermore, a response of $>100$ days after treatment with Rova-T has been shown in PDX-models of melanoma and small cell ovarium carcinoma. ${ }^{33}$ 


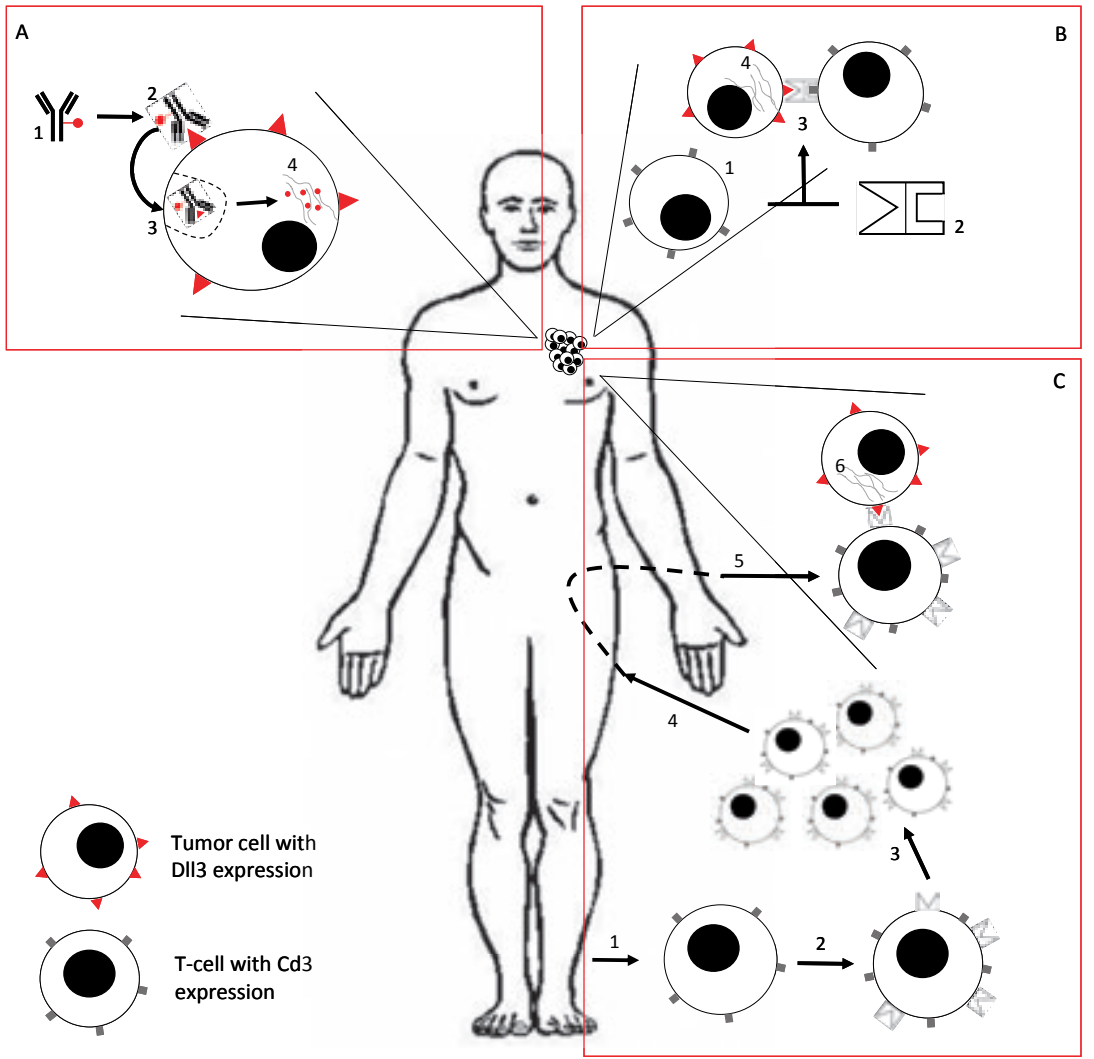

Figure 6.2 Three technologies for DII3 targeted therapy. A) Antibody-drug conjugate (rovalpituzumabtesirine, Rova-T). (1) A toxin is coupled to a Dll3-antibody. (2) After injection, the antibody will bind to DII3 on the tumor cell and (3) the conjugate will be internalized. (4) The toxin induces cell damage, resulting in cell death. B) BiTE ${ }^{\circledR}$ technique. (1) The cytotoxic T-cell is not able to recognize and bind the tumor cell. (2) A bi-specific antibody is infused, consisting of a T-cell specific antibody and a tumor cell specific antibody (anti-DII3). (3) Binding of the bi-specific antibody to both the cytotoxic T-cell and the tumor cell, results in activation of the T-cell, and (4) Iysis of the tumor cell. C) CAR-T technique. (1) T-cells are obtained from the patient by leukaferesis. (2) T-cells are genetically modified to express a tumor cell specific antibody (antiDII3). (3) After expansion, T-cells are infused back (4) into the patient. (5) The modified T-cells can bind to the tumor cells, and (6) induce lysis of the tumor cells.

The first phase 1 study with Rova-T showed promising results. ${ }^{7} 65$ pre-treated patients, mostly SCLC and some LCNEC, were treated with increasing doses of Rova-T. 11/65 (17\%) patients had an objective response and 35/65 (54\%) stable disease. Dll3 expression could be determined on histologic material in 39 patients, any cytoplasmic and/or membranous staining was considered positive. In the group with a high 
percentage ( $\geq 50 \%$ ) Dll3 positive tumor cells, an objective response was seen in 10/29 (35\%) patients and stable disease in 16/29 (55\%). In patients with no or low Dll3 expression, 6/10 (60\%) patients had stable disease and no objective responses were observed. Ten patients in this study had long-lasting stable disease after 2 or 3 doses of Rova-T ( 8 patients $>6$ months and two patients $>12$ months). ${ }^{7}$ In this phase 1 study, a maximum tolerated dose of Rova-T of $0.4 \mathrm{mg} / \mathrm{kg}$ every 3 weeks was found. However, unacceptable late toxic effects were observed at this dose. Therefore, recommended dose for further research was two cycles of $0.3 \mathrm{mg} / \mathrm{kg}$ every 6 weeks. ${ }^{7}$

After this promising phase 1 trial, analysis of the first phase 2 trial (TRINITY, N=339) showed less effectivity. ${ }^{39}$ In this study, SCLC patients with a DII3 positive tumor ( $\left.\geq 25 \%\right)$, pre-treated with at least two lines of chemotherapy, were included. An overall response rate of $12.4 \%$ was observed and median OS was 5.6 months ( $95 \%$ confidence interval ( $\mathrm{Cl}$ ) 4.9-6.1 months). In the subgroup with a high percentage of DII3 positive tumors cells $(\geq 75 \%)$ response rate was $14.3 \%$ and median OS 5.7 months $(95 \% \mathrm{Cl} 4.9$ 6.7 months). ${ }^{40}$ In this study, grade 3-5 drug related adverse events were seen in 169/339 (50\%) patients (thrombocytopenia (13\%), photosensitivity (7\%), pleural effusion (6\%) and anemia (6\%)). ${ }^{40}$ Interim analysis by the independent data monitoring committee of two phase 3 studies (TAHOE and MERU) demonstrated inferior survival in the Rova-T group compared to the control arm. ${ }^{41,42}$ Both studies were closed for inclusion and development of Rova-T was halted by Abbvie. ${ }^{41,42}$ Preliminary results of a phase $1 / 2$ study regarding safety profile in non-pulmonary carcinoma showed adverse events in 22/31 (84\%) of patients with melanoma, medullar thyroid carcinoma, glioblastoma and different types of neuroendocrine carcinoma. Three of the patients $(10 \%)$ had grade 3 or higher adverse events. ${ }^{43}$ No results on effectivity of this study have been presented yet. Table 6.3 gives an overview of all registered ongoing clinical trials for Rova-T. In addition to Rova-T monotherapy, a combination therapy with subtherapeutic doses of Rova-T and anti-programmed death (Pd)-1 therapy (ABBV-181) is investigated (Table 6.3). ${ }^{44}$ 


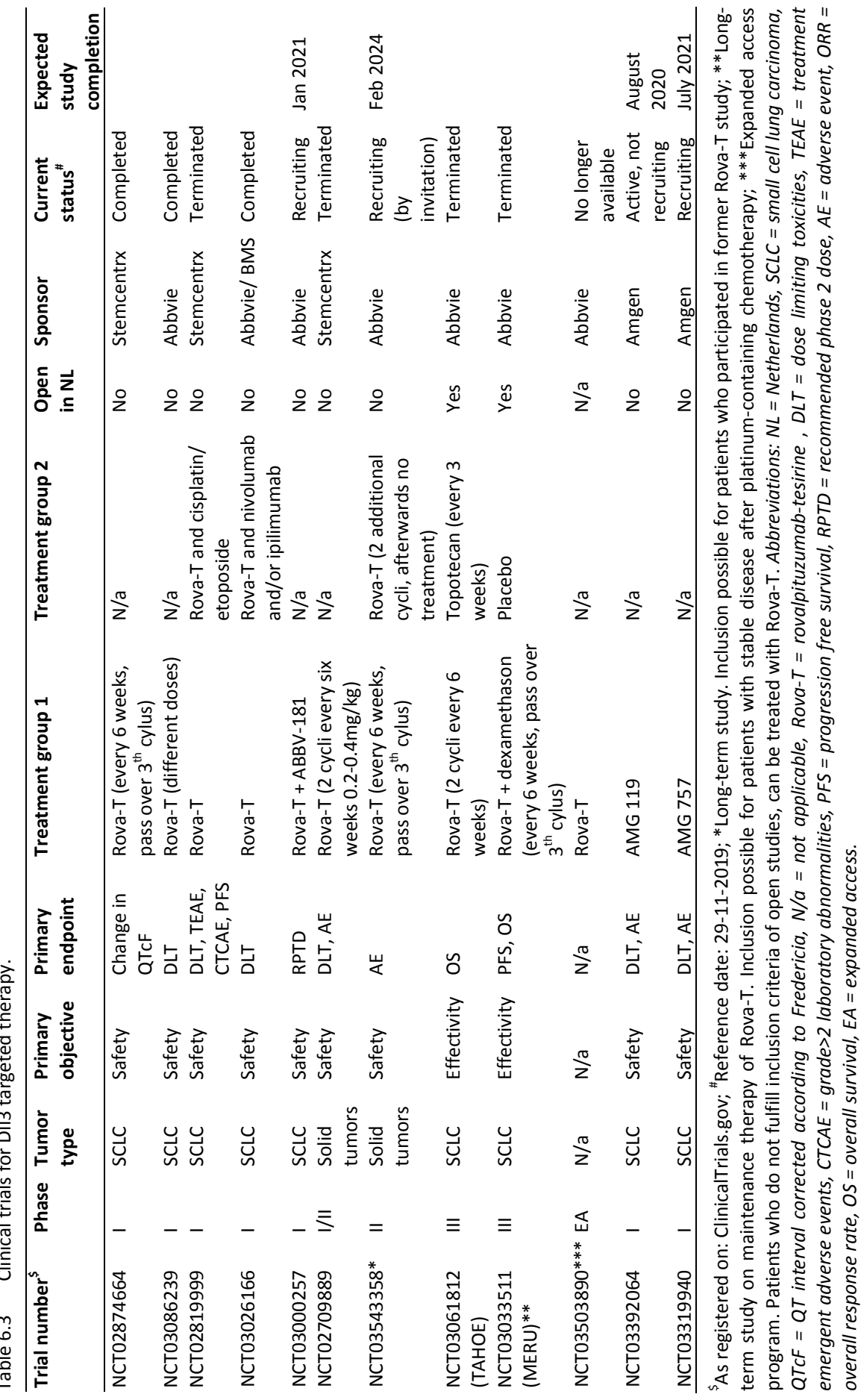




\section{$\mathrm{BiTE}^{\circledR}$}

Immunotherapy is extensively used in oncology, activating the immune system to fight tumor cells. However, tumor cells are not always recognized by the immune system (e.g. lack of major histocompatibility complex-I (MHC-I) proteins) and tumor cells might inhibit the immune system (e.g. expression of $\mathrm{Pd}-\mathrm{l1}$ ). These problems could be bypassed with a Bispecific T-cell Engaging (BiTE $\left.{ }^{\oplus}\right)$ technique whereby a T-cell specific antibody (anti-Cd3) is coupled to a tumor specific antibody (e.g. anti-Cd19 or anti-

DII3). ${ }^{45,46}$ Binding of this bispecific antibody to both the tumor cell and the cytotoxic T-cell will activate the T-cell, which will execute lysis of the tumor cell (Figure 6.2B). Experience with this technique has mainly been gained in the field of hematology and the BiTE ${ }^{\circledR}$ blinatumomab is already used as treatment for acute lymphatic leukemia. ${ }^{45}$ AMG757 is a BiTE ${ }^{\circledR}$ antibody construct with the tumor specific antibody targeting DII3. Reduction of tumor growth has been shown after exposure to this drug in vitro and in vivo. Furthermore, AMG757 had a tolerable toxicity profile in one toxicologic study. A phase 1 study is ongoing to reveal safety and effectivity of AMG757 in SCLC (Table 6.3). ${ }^{47}$ Recently, preclinical data of another DII3/Cd3 IgG-like T cell engager construct were presented. A dose-dependent lysis of cell lines and anti-tumor activity in PDXmodels has been observed. Phase 1 studies are planned to be initiated after further development of this drug. ${ }^{48}$

\section{CAR-T}

For Chimeric Antigen Receptor T-cell (CAR-T) therapy, T-cells of the patient are obtained by leukaferesis. ${ }^{49}$ Subsequently, the T-cells are genetically modified to express a tumor cell specific antibody (e.g. anti-Cd19, anti-DII3). The T-cells are multiplied and the modified and activated T-cells are infused back into the patient. Binding of a cytotoxic CAR-T cell to the tumor cell will result in lysis of the tumor cell (Figure 6.2C). Currently, tisagenlecleucel (acute lymphatic leukemia) and axicabtagene ciloleucel (diffuse large cell B-cell lymphoma) have been registered. ${ }^{49,50}$ The most common adverse event of CAR-T is 'cytokine release syndrome'. Furthermore, high toxicity has been described in cases where the antigen is also present, even in low levels, in normal tissue. ${ }^{49}$ AMG119 is a CAR-T, targeting DII3. Both in vitro and in vivo, anti-tumor activity has been shown with a clear proliferation of CAR-T cells and production of cytokines in the presence of Dll3 positive cells. Currently, a phase 1 study for AMG119 is open for inclusion. ${ }^{47}$ 


\section{Conclusion}

The expression of DII3 in the vast majority of SCLC and LCNEC in combination with very limited expression in normal tissue makes DII3 a promising therapeutic target. After initial enthusiasm for Rova-T, subsequent studies have shown disappointing results. Furthermore, a relevant cut-off value for percentage of positive DIl3 cells has not been defined yet and it is unclear if type of staining has a predictive value for targeted therapy. Results of enrolling and recently closed phase I-III studies are being awaited. Only then, definite conclusions can be drawn considering the effectivity of Rova-T. BiTE $^{\circledR}$ and CAR-T techniques might be an alternative for Dll3 targeted therapy, because of different mechanisms of action and good results in hematological malignancies. First clinical trials are being awaited and in case of positive results, further studies should be initiated. 


\section{References}

1. Derks JL, Hendriks LE, Buikhuisen WA, et al. Clinical features of large cell neuroendocrine carcinoma: a population-based overview. Eur Respir J 2016;47:615-624.

2. Asamura $\mathrm{H}$, Kameya $\mathrm{T}$, Matsuno $\mathrm{Y}$, et al. Neuroendocrine neoplasms of the lung: a prognostic spectrum. J Clin Oncol 2006;24:70-76.

3. NVALT. Small Cell Lung Carcinoma, national guideline the Netherlands, version 1.0. 10-05-2011. Available at www.oncoline.nl.

4. Derks JL, van Suylen RJ, Thunnissen E, et al. Chemotherapy for pulmonary large cell neuroendocrine carcinomas: does the regimen matter? Eur Respir J 2017;49.

5. NVALT. Non-Small Cell Lung Carcinoma, national guideline the Netherlands, version 2.3 18-12-2015. Available at www.oncoline.nl.

6. Horn L, Mansfield AS, Szczesna A, et al. First-Line Atezolizumab plus Chemotherapy in Extensive-Stage Small-Cell Lung Cancer. N Engl J Med 2018;379:2220-2229.

7. Rudin CM, Pietanza MC, Bauer TM, et al. Rovalpituzumab tesirine, a DLL3-targeted antibody-drug conjugate, in recurrent small-cell lung cancer: a first-in-human, first-in-class, open-label, phase 1 study. Lancet Oncol 2017;18:42-51.

8. Saunders LR, Bankovich AJ, Anderson WC, et al. A DLL3-targeted antibody-drug conjugate eradicates high-grade pulmonary neuroendocrine tumor-initiating cells in vivo. Sci Transl Med 2015;7:302ra136.

9. D'Souza B, Miyamoto A, Weinmaster G. The many facets of Notch ligands. Oncogene 2008;27:51485167.

10. Ladi E, Nichols JT, Ge W, et al. The divergent DSL ligand DIl3 does not activate Notch signaling but cell autonomously attenuates signaling induced by other DSL ligands. J Cell Biol 2005;170:983-992.

11. Deng SM, Yan XC, Liang L, et al. The Notch ligand delta-like 3 promotes tumor growth and inhibits Notch signaling in lung cancer cells in mice. Biochem Biophys Res Commun 2017;483:488-494.

12. Chapman G, Sparrow DB, Kremmer E, et al. Notch inhibition by the ligand DELTA-LIKE 3 defines the mechanism of abnormal vertebral segmentation in spondylocostal dysostosis. Hum Mol Genet 2011;20:905-916.

13. Jiang T, Collins BJ, Jin N, et al. Achaete-scute complex homologue 1 regulates tumor-initiating capacity in human small cell lung cancer. Cancer Res 2009;69:845-854.

14. Henke RM, Meredith DM, Borromeo MD, et al. Ascl1 and Neurog2 form novel complexes and regulate Delta-like3 (DII3) expression in the neural tube. Dev Biol 2009;328:529-540.

15. Nakakura EK, Sriuranpong VR, Kunnimalaiyaan M, et al. Regulation of neuroendocrine differentiation in gastrointestinal carcinoid tumor cells by notch signaling. J Clin Endocrinol Metab 2005;90:4350-4356.

16. Sriuranpong $\mathrm{V}$, Borges $\mathrm{MW}$, Strock $\mathrm{CL}$, et al. Notch signaling induces rapid degradation of achaete-scute homolog 1. Mol Cell Biol 2002;22:3129-3139.

17. Kunnimalaiyaan $\mathrm{M}$, Chen $\mathrm{H}$. Tumor suppressor role of Notch-1 signaling in neuroendocrine tumors. Oncologist 2007;12:535-542.

18. Linnoila RI, Zhao B, DeMayo JL, et al. Constitutive achaete-scute homologue-1 promotes airway dysplasia and lung neuroendocrine tumors in transgenic mice. Cancer Res 2000;60:4005-4009.

19. Espinoza I, Miele L. Notch inhibitors for cancer treatment. Pharmacol Ther 2013;139:95-110.

20. Maemura K, Yoshikawa H, Yokoyama K, et al. Delta-like 3 is silenced by methylation and induces apoptosis in human hepatocellular carcinoma. Int J Oncol 2013;42:817-822.

21. Tanaka K, Isse K, Fujihira T, et al. Prevalence of Delta-like protein 3 expression in patients with small cell lung cancer. Lung Cancer 2018;115:116-120.

22. Saito M, Saito K, Shiraishi K, et al. Identification of candidate responders for anti-PD-L1/PD-1 immunotherapy, Rova-T therapy, or EZH2 inhibitory therapy in small-cell lung cancer. Mol Clin Oncol 2018;8:310-314.

23. Huang RSP, Holmes BF, Powell C, et al. Delta-like Protein 3 Prevalence in Small Cell Lung Cancer and DLL3 (SP347) Assay Characteristics. Arch Pathol Lab Med 2019;143(11):1373-1377. 
24. Roy S, Martinez K, Dilip A, et al. DLL3 analysis of circulating tumor cells predict treatment in phase 1 rova-T study in small cell lung cancer (Abstract \#3721). AACR. Washington, DC: 2017;Abstracts 30635947.

25. Furuta M, Sakakibara-Konishi J, Kikuchi H, et al. Analysis of DLL3 and ASCL1 in Surgically Resected Small Cell Lung Cancer (HOT1702). Oncologist 2019;24:e1172-e1179.

26. Hermans BCM, Derks JL, Thunnissen E, et al. DLL3 expression in large cell neuroendocrine carcinoma (LCNEC) and association with molecular subtypes and neuroendocrine profile. Lung Cancer 2019;138:102-108.

27. Brcic L, Kuchler C, Eidenhammer S, et al. Comparison of four DLL3 antibodies performance in high grade neuroendocrine lung tumor samples and cell cultures. Diagn Pathol 2019;14:47.

28. Xie H, Boland JM, Maleszewski JJ, et al. Expression of delta-like protein 3 is reproducibly present in a subset of small cell lung carcinomas and pulmonary carcinoid tumors. Lung Cancer 2019;135:73-79.

29. Messaritakis I, Nikolaou M, Koinis F, et al. Characterization of DLL3-positive circulating tumor cells (CTCs) in patients with small cell lung cancer (SCLC) and evaluation of their clinical relevance during front-line treatment. Lung Cancer 2019;135:33-39.

30. Sharma SK, Pourat J, Abdel-Atti D, et al. Noninvasive Interrogation of DLL3 Expression in Metastatic Small Cell Lung Cancer. Cancer Res 2017;77:3931-3941.

31. Xie H, Isse K, Sun Y, et al. Delta-like protein 3 expression in Merkel cell carcinoma (Abstract \#3171). AACR. Atlanta: 2019;Part B: Abstract 2749-5314.

32. Koshkin VS, Garcia JA, Reynolds J, et al. Transcriptomic and Protein Analysis of Small-cell Bladder Cancer (SCBC) Identifies Prognostic Biomarkers and DLL3 as a Relevant Therapeutic Target. Clin Cancer Res 2019;25:210-221.

33. Saunders LR, Williams SA, Bheddah S, et al. Expression of DLL3 in metastatic melanoma, glioblastoma and high-grade extrapulmonary neuroendocrine carcinomas as potential indications for rovalpituzumab tesirine (Rova-T; SC16LD6.5), a delta-like protein 3 (DLL3)-targeted antibody drug conjugate (ADC) (Abstract \#3093). AACR. Washington, DC: 2017;77.

34. Puca L, Gavyert K, Sailer V, et al. Delta-like protein 3 expression and therapeutic targeting in neuroendocrine prostate cancer. Sci Transl Med 2019;11.

35. Spino M, Kurz SC, Chiriboga L, et al. Cell Surface Notch Ligand DLL3 is a Therapeutic Target in Isocitrate Dehydrogenase-mutant Glioma. Clin Cancer Res 2019;25:1261-1271.

36. Pancewicz-Wojtkiewicz J, Eljaszewicz A, Kowalczuk O, et al. Prognostic significance of Notch ligands in patients with non-small cell lung cancer. Oncol Lett 2017;13:506-510.

37. Vranic S, Palazzo J, Sanati S, et al. Potential Novel Therapy Targets in Neuroendocrine Carcinomas of the Breast. Clin Breast Cancer 2019;19:131-136.

38. Tiberghien AC, Levy JN, Masterson LA, et al. Design and Synthesis of Tesirine, a Clinical Antibody-Drug Conjugate Pyrrolobenzodiazepine Dimer Payload. ACS Med Chem Lett 2016;7:983-987.

39. Carbone D, Morgensztern D, Moulec SL, et al. Efficacy and safety of rovalpituzumab tesirine in patients with DLL3-expressing, $\geq 3$ rd line small cell lung cancer: Results from the phase 2 TRINITY study. ASCO Annual Meeting; Abstract \#8507 2018.

40. Morgensztern D, Besse B, Greillier L, et al. Efficacy and Safety of Rovalpituzumab Tesirine in Third-Line and Beyond Patients with DLL3-Expressing, Relapsed/Refractory Small-Cell Lung Cancer: Results From the Phase II TRINITY Study. Clin Cancer Res 2019;25(23):6958-6966.

41. Phase 3 Trial of Rova-T as Second-line Therapy for Advanced Small-Cell Lung Cancer (TAHOE Study) Halted [press release]. AbbVie, North Chicago; 2018.

42. AbbVie Discontinues Rovalpituzumab Tesirine (Rova-T) Research and Development Program [press release]. Abbvie, North Chicago; 2019.

43. Aggarwal R, Mansfield A, Beltran $\mathrm{H}$, et al. Preliminary Safety and Efficacy of Rovalpituzumab Teserine in Patients With Delta-Like Protein 3-Expressing Advanced Solid Tumors (Abstract 436 PD). Annals of Oncology 2017;28, suppl_5:v142-v157.

44. Vitorno P, Chuang C-H, Moore C, et al. Rovalpituzumab tesirine enhances the anti-tumor efficacy of PD1 blockade in a murine model of small cell lung cancer with endogenous DLL3 expression (Abstract \#3950). AACR. Atlanta: 2019;Part B: Abstracts 2749-5314. 
45. Yuraszeck T, Kasichayanula S, Benjamin JE. Translation and Clinical Development of Bispecific T-cell Engaging Antibodies for Cancer Treatment. Clin Pharmacol Ther 2017;101:634-645.

46. Wu Z, Cheung NV. T cell engaging bispecific antibody (T-BsAb): From technology to therapeutics. Pharmacol Ther 2018;182:161-175.

47. Giffin M, Cooke K, Lobenhofer E, et al. Targeting DLL3 with AMG 757, a BiTE ${ }^{\circledR}$ Antibody Construct, and AMG 119, a CAR-T, for the Treatment of SCLC. J Thorac Oncol 2018;13:Supplement.

48. Hipp S, Voynov V, Drobits-Handl B, et al. A novel T-cell engaging bispecific antibody induces specific and efficacious lysis of small cell lung cancer cells in vitro and potent $T$ cell re-directed anti-tumor activity in vivo (Abstract \#549). AACR. Atlanta: 2019; Part A: Abstracts 1-2748.

49. Minutolo NG, Hollander EE, Powell DJ, Jr. The Emergence of Universal Immune Receptor T Cell Therapy for Cancer. Front Oncol 2019;9:176.

50. Grigor EJM, Fergusson D, Kekre N, et al. Risks and Benefits of Chimeric Antigen Receptor T-Cell (CAR-T) Therapy in Cancer: A Systematic Review and Meta-Analysis. Transfus Med Rev 2019;33(2):98-110. 



\section{Chapter 7}

DIl3 expression in large cell neuroendocrine carcinoma (LCNEC) and association with molecular subtypes and neuroendocrine profile

B.C.M. Hermans, J.L. Derks, E. Thunnissen, R. J. van Suylen, M.A. den Bakker, H.J.M. Groen, E.F. Smit, R.A. Damhuis, E.C. van den Broek, PALGA-group, A. Ruland, E.J.M. Speel*, A-M.C. Dingemans*

* contributed equally to this work Lung Cancer 2019;138:102-108 


\section{Abstract}

\section{Background}

For stage IV pulmonary large cell neuroendocrine carcinoma (LCNEC), the only therapeutic option is palliative chemotherapy. DII3 is a new therapeutic target, which seems to be often expressed in SCLC and LCNEC. It has recently been reported that DLL3 mRNA expression is particularly upregulated in the LCNEC subgroup with STK11/KEAP1 and TP53 co-mutations, in contrast to lower expression levels in RB1 and TP53 co-mutated LCNEC. Our aim was to investigate DII3 protein expression in stage IV LCNEC and correlate data with mutational profiles (i.e. STK11/KEAP1/RB1), immunostaining results ( $p R b$, neuroendocrine markers) and clinical characteristics.

\section{Methods}

Immunohistochemical analysis for DII3 (SC16.65) and Ascl1 (SC72.201) was performed on 94 and 51 FFPE tissue sections, respectively, of pathologically reviewed stage IV LCNEC. DII3 and Ascl1 were scored positive if $\geq 1 \%$ of the tumor cells showed cytoplasmic/membranous or dotlike (DII3) or nuclear (Ascl1) immunostaining. Data were correlated with available sequencing (TP53, RB1, STK11, KEAP1), immunostaining ( $p R b$, neuroendocrine markers) and clinical data.

\section{Results}

DII3 was expressed in 70/94 (74\%) LCNEC, 56 (80\%) of which showed cytoplasmic/membranous staining. Median H-score was 55 (interquartile range 0-160). DIl3 staining was not different in $\mathrm{pRb}$ immunohistochemistry negative and positive patients (DII3+ in 53/70 (76\%) vs. 14/21 (67\%), $\mathrm{p}=0.409)$ or RB1 mutated and wildtype patients (DII3+ in 27/34 (79\%) vs. 23/33 (70\%), p=0.361). Nevertheless, 6/6 (100\%) STK11 mutated, 10/11 (91\%) KEAP1 mutated and 9/9 (100\%) TP53 wildtype tumors were Dll3+. Furthermore, DII3 expression was associated with expression of Ascl1 and at least 2 out of 3 neuroendocrine markers.

\section{Conclusion}

The high percentage (74\%) of DII3 expression in stage IV LCNEC denotes the potential of DII3 targeted therapy in this patient group. 


\section{Introduction}

Large cell neuroendocrine carcinoma (LCNEC) and small cell lung carcinoma (SCLC) are aggressive neuroendocrine tumors with poor survival rates. ${ }^{1-3}$ For stage IV SCLC, treatment has not advanced significantly over the last decades and consists of palliative chemotherapy. The same applies to stage IV LCNEC, were no standard treatment exists and palliative chemotherapy with SCLC and non-small cell lung cancer (NSCLC) regimens are both deemed appropriate. ${ }^{4}$ Recently, targeted therapy focusing on delta like protein 3 (DII3) has received attention to improve outcomes for SCLC and LCNEC. ${ }^{5}$

DII3 is part of the Notch family including four Notch receptors (Notch1-4) and five transmembrane ligands, coded by jagged (JAG1 and JAG2) and delta-like (DLL1, DLL3 and DLL4) gene families. DII3 is an important link in the achaete-scute complex-like 1 (Ascl1) - DII3 - Notch1 pathway. The supposed exclusive function of DII3 is inhibition of Notch1, in contrast to DII1 and Dll4 which have both inhibitory and stimulatory effects on the Notch pathway. ${ }^{6-8}$ DII3 has been reported to be a downstream target of Ascl1, a transcription factor critical for development of pulmonary neuroendocrine cells in the developing lung. ${ }^{9,10}$ Therefore, activation of Ascl1 will result in Dll3 upregulation and increased inhibition of Notch1. ${ }^{5}$ In addition, Notch1 has been described to be a negative regulator of Ascl1. ${ }^{11,12}$ Apparently, the Ascl1 - DII3 - Notch1 pathway is nonlinear, and a change in expression of one of the proteins influences the others. In some tumor types, Notch pathway activation (i.e. Notch1 upregulation or Ascl1 downregulation) results in oncogenic stimulation and tumor growth. ${ }^{13,14}$ However, both Notch1 inhibition and Ascl1 upregulation have shown to result in development of neuroendocrine neoplasms. ${ }^{9,14-16}$

Two main molecular subtypes of LCNEC have been identified by next generation sequencing (NGS) studies. The first subtype has mutations in TP53 and STK11 and/or KEAP1 (NSCLC-like), whereas the second subtype has mutations of TP53 and RB1 (a hallmark of SCLC). ${ }^{17-19}$ The subclassification could also be made based on $\mathrm{pRb}$ immunohistochemistry (IHC) expression, classifying tumors with loss of $p R b$ as SCLClike. $^{20}$ A small subset of LCNEC with carcinoid-like features has also been identified, enriched for MEN1 mutations. ${ }^{17}$ Our recent study emphasized clinical relevance of the two main LCNEC subtypes, by showing a worse survival for NSCLC-like LCNEC patients treated with platinum-etoposide compared to NSCLC-regimen whereas no difference was found for the SCLC-like subtype. ${ }^{20}$ Interestingly, a recent study identified that STK11/KEAP1 mutated LCNEC have a Notch $1^{\text {Low }} / D L L 3^{\text {High }} / A S C L 1^{\text {High }}$ RNA expression signature while the TP53/RB1 mutated LCNEC had a Notch1 $1^{\text {High }} / D L L 3^{\text {Low }} / A S C L 1^{\text {Low }}$ 
signature. ${ }^{18}$ Hence, LCNEC molecular subtypes may be related to DII3 expression also at protein level.

Only limited data on prevalence of DII3 and no data on type of staining, percentage of positive cells within each sample or survival related to DII3 expression in LCNEC is available. In this study, we assessed DII3 expression by IHC in a cohort of 94 patients with well characterized and molecular profiled stage IV LCNEC. In addition, the association of DII3 status with mutational status (RB1, TP53, KEAP1, STK11) and IHC expression of $\mathrm{pRb}$, Ascl1, Ttf1 and neuroendocrine markers was investigated.

\section{Materials and methods}

\section{Patient selection}

All data for this retrospective population-based study were retrieved from the Netherlands Cancer Registry and Netherlands Pathology Registry (PALGA) as described before (2003-2012). ${ }^{21,22}$ Clinical data was updated until 2015 and comprised age, gender, overall survival (OS) and progression free survival (PFS).

The study protocol was approved by the medical ethical committee of the Maastricht University Medical Centre (METC azM/UM 14-4-043).

\section{Pathologic material}

Panel consensus pathology revision was performed as described earlier for 232 stage IV LCNEC. Samples were evaluated for neuroendocrine morphology (organoid nesting, palisading, rosettes or trabeculae), mitotic index, necrosis, and neuroendocrine differentiation using IHC for at least one neuroendocrine marker (NE-marker). Diagnosis was confirmed in patients meeting the WHO-criteria. ${ }^{23}$ If strict WHO-criteria were not met, but the pathologists found it likely that LCNEC was the correct diagnosis, an exception was made as described earlier. ${ }^{24,25}$

IHC for pRb (13A10) were available for the majority of the patients. ${ }^{20}$ Furthermore, for most patients, IHC results for Ttf1 and NE-markers (Cd56, Chromogranin A and Synaptophysin) were present. In case of absence of one or two of the NE-markers, extra immunostaining was performed if tissue was available. NE-markers were scored as negative, weakly $(+)$, moderately $(++)$ or strongly $(+++)$ positive, as described before. ${ }^{25}$ 
Immunohistochemistry

\section{DII3}

We performed DII3 immunostaining on tumors with confirmed LCNEC diagnosis if FFPE blocks with sufficient available tumor tissue were available. IHC was performed on 3 $\mu \mathrm{m}$ thick tissue sections using SC16.65 antibody $(3 \mu \mathrm{g} / \mathrm{ml})$ and a IHC DAKO FLEX Mouse linker protocol (both provided by Abbvie). A low $\mathrm{pH}$ antigen retrieval was used. A positive control (HEK-293T.hDLL3) and a negative control (HEK-293T) were included (also provided by Abbvie). ${ }^{5}$ Immunostainings were evaluated by two investigators (BH and EJS), who were blinded for all clinical, histopathological and mutational data. Selected samples were discussed with K. Isse (pathologist, Abbvie) for confirmation. Samples were scored as positive ( $\geq 1 \%$ positive tumor cells) or negative $(<1 \%)$ for Dll3. ${ }^{26}$ Scoring for positive samples was further specified by intensity (low (1), intermediate (2) or high (3)), percentage of positive cells and type of staining: more diffuse staining of both cytoplasm and membrane was scored as cytoplasmic/membranous (Figure 7.1A), and a more punctuated perinuclear pattern was scored as dotlike (Figure 7.1B). H-score was calculated per case by multiplying intensity with percentage of positive cells.

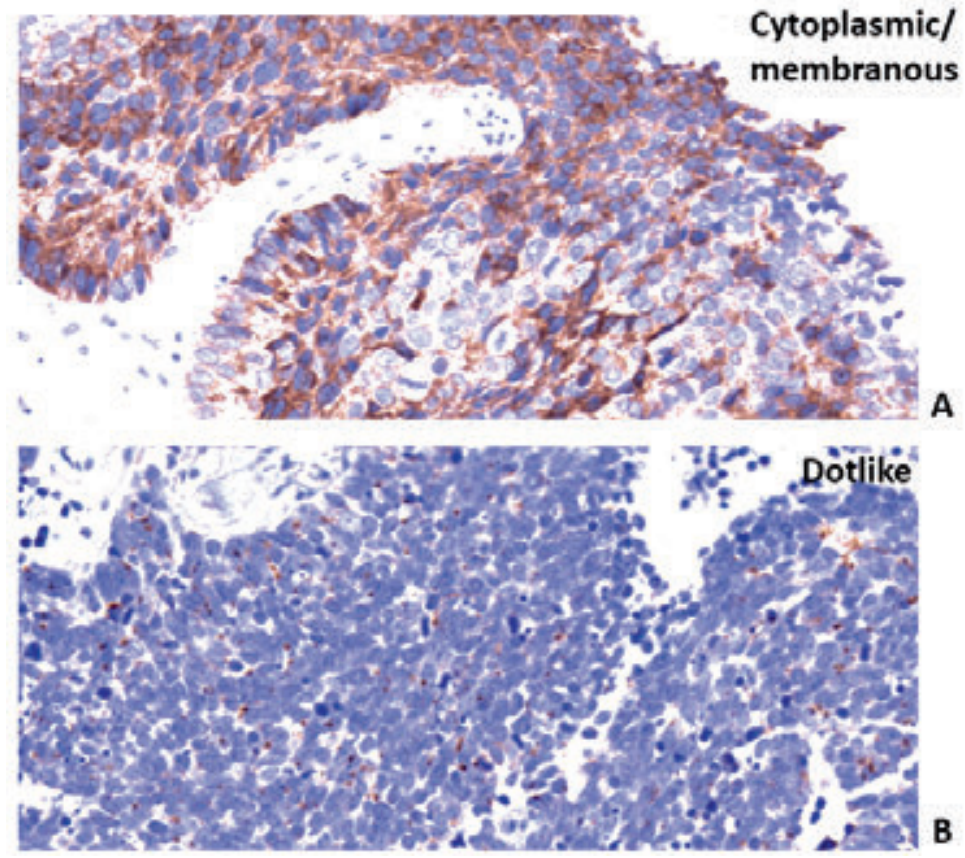

Figure 7.1 Representative samples of Dll3 immunohistochemistry on LCNEC tumors. A) Combined cytoplasmic and membranous staining. B) Perinuclear dotlike staining. 
Ascl1

IHC for Ascl1 was performed if sufficient tumor material was available. IHC was performed on $3 \mu \mathrm{m}$ thick slides using SC72.201 antibody $(1 \mu \mathrm{g} / \mathrm{ml})$ and the IHC DAKO FLEX+ mouse linker protocol (both provided by Abbvie). A low pH antigen retrieval was used. An Ascl1 positive cell line and SCLC sample were used as positive controls. The negative controls were an Ascl1 negative cell line and SCLC sample. Nuclear staining was evaluated by two investigators (BH and EJS), who were blinded for all clinical, histopathological and mutational data. Samples were scored positive $(\geq 1 \%$ positive tumor cells) or negative $(<1 \%)$. Scoring for positive samples was further specified by intensity (low (1), intermediate (2) or high (3)) and percentage of positive cells. H-score was calculated per case by multiplying intensity with percentage of positive cells.

\section{Mutational analysis}

DNA was isolated from available FFPE tissue blocks and targeted NGS was performed for RB1, KEAP1, STK11 and TP53, as described earlier. ${ }^{20}$ All co-mutated RB1 and TP53 samples as well as pRb IHC negative/TP53 mutated samples were classified as SCLC-like. All other samples were classified as non SCLC-like. Since the third LCNEC subgroup with carcinoid-like morphology and MEN1 mutations is very small, this subgroup was not further addressed in this study.

\section{Statistics}

All analyses were performed using SPSS (version 25 for Windows, Armonk, NY: IBM Corp.). Association of DII3 status (DII3+ or DII3-) with gender, mutational status (RB1, STK11, KEAP1 and TP53 mutation), number of positive NE-markers (1 or $\geq 2$ ) and positive immunostaining for $\mathrm{pRb}$, Ttf1 and Ascl1, was investigated with chi-squared test or Fisher's Exact Test. Association between Dll3 $\mathrm{H}$-score and Ascl1 H-score was investigated with Spearman correlation. Differences in median age in Dll3+ versus DII3patients and differences in median DII3 $\mathrm{H}$-score in patients with $1 \mathrm{NE}$-marker versus $\geq 2$ NE-markers were tested with Mann Whitney $U$ test. Differences in median DIl3 H-score for different intensities of NE-markers were analyzed with Kruskal-Wallis test. Median overall survival (OS) was evaluated by Kaplan Meier analysis and differences in survival were tested for significance with Log-Rank test for DII3 positive and negative staining. Results are presented as hazard ratios (HR) with $95 \%$ confidence intervals $(\mathrm{Cl})$. $\mathrm{P}<0.05$ was considered significant. 


\section{Results}

\section{Patient characteristics}

Dll3 immunostaining was performed in 94 out of 148 patients with consensus based confirmed LCNEC (Supplemental Figure S7.A). Mean age at diagnosis was 63 years (range $34-82$ years). A total of $61 \%$ of patients were male (Table 7.1). Staining for three NE-markers (Cd56, Synaptophysin, Chromogranin A), pRb and Ttf1 was available in 91, 91 and 83 samples, respectively. NGS for STK11, KEAP1, RB1 and TP53 was available for 67 patients (Supplemental Figure S7.A). For Ascl1 IHC, only in 51 cases sufficient tumor tissue was available.

Table 7.1 DII3 expression in stage IV LCNEC.

\begin{tabular}{|c|c|c|c|}
\hline & Dll3+ & DII3- & p-value \\
\hline Total $(\mathrm{N}=94)$ & $70(74 \%)$ & $24(26 \%)$ & \\
\hline \multicolumn{4}{|l|}{$\%$ positive cells } \\
\hline$\geq 1 \%$ & $70(74 \%)$ & - & \\
\hline$\geq 25 \%$ & $62(66 \%)$ & - & \\
\hline$\geq 50 \%$ & $51(54 \%)$ & - & \\
\hline$\geq 75 \%$ & $35(37 \%)$ & - & \\
\hline \multicolumn{4}{|l|}{ H-score $(\mathrm{N}=70)$} \\
\hline$\leq 100$ & $33(47 \%)$ & - & \\
\hline $101-\leq 200$ & $21(30 \%)$ & - & \\
\hline $201-\leq 300$ & $16(23 \%)$ & - & \\
\hline \multicolumn{4}{|l|}{ Type of staining $(\mathrm{N}=70)$} \\
\hline Mainly dotlike & $14(20 \%)$ & - & \\
\hline Mainly cytoplasmic/ membranous & $56(80 \%)$ & - & \\
\hline \multicolumn{4}{|l|}{ Patient characteristics } \\
\hline \multicolumn{4}{|l|}{ Gender } \\
\hline Male $(\mathrm{N}=57)$ & $44(77 \%)$ & $13(23 \%)$ & \multirow{2}{*}{$0.45^{*}$} \\
\hline Female $(\mathrm{N}=37)$ & $26(70 \%)$ & $11(30 \%)$ & \\
\hline Age (median, IQ range) & $62(55-71)$ & $65(60-71)$ & $0.28 * *$ \\
\hline
\end{tabular}

* Chi-square; ** Mann-Whitney $\mathrm{U}$ test. IQ range = interquartile range.

\section{DII3 IHC}

Dll3 staining was positive ( $\geq 1 \%$ of tumor cells positive) in $70 / 94(74 \%)$ samples (Table 7.1). Of the 94 patients, 62 (66\%) had Dll3 staining in $\geq 25 \%$ of tumor cells, $51(54 \%)$ in $\geq 50 \%$ and 35 (37\%) in $\geq 75 \%$. Median $\mathrm{H}$-score was 55 (interquartile range (IQ) $0-160$ ). Of the 70 DII3 positive samples, $56(80 \%)$ had mainly cytoplasmic/membranous staining and only 14 (20\%) had mainly perinuclear dotlike staining (Table 7.1, Figure 7.1). Isolated membranous staining was not observed. DII3 expression was not associated with gender or age (Table 7.1). A trend towards more Dll3 positivity in Ttf1 positive 
LCNEC compared to Ttf1 negative LCNEC was observed (52/63 (83\%) vs. 12/20 (60\%), $\mathrm{p}=0.063$ ) (Figure 7.2, Supplemental Table S7.A). OS in DII3+ patients was comparable to DIl3- patients (6.9 months (95\% confidence interval (CI) 5.1-8.7) vs. 6.1 months $(95 \% \mathrm{Cl}$ 4.3-7.9), HR 1.00, p=1.00) (Supplemental Figure S7.B).

\section{DII3 in relation to LCNEC mutational subtypes}

No difference was found between pRb IHC positive and negative groups (DII3+ in 14/21 (67\%) vs. 53/70 (76\%), $p=0.41$ ) (Figure 7.2, Supplemental Table S7.A). Also, no difference was found between RB1 wildtype and RB1 mutated subgroups (DII3+ in $23 / 33(70 \%)$ vs. $27 / 34$ (79\%), $p=0.36)$. After classification of samples by combining information on TP53 and RB1 mutation and pRb IHC expression, 67 SCLC-like and 24 non SCLC-like cases were identified. No difference in DII3 expression was found in the two subgroups (DII3+ in 49/67 (73\%) vs. 18/24 (75\%), p=0.86). Regarding additional mutational analysis, 6/6 (100\%) STK11 mutated vs. 44/61 (72\%) STK11 wildtype $(p=0.33)$ and 10/11 (91\%) KEAP1 mutated vs. 40/56 (71\%) KEAP1 wildtype tumors ( $p=0.27)$ were DII3 positive. Furthermore, 9/9 (100\%) TP53 wildtype tumors were DII3 positive vs. 41/58 (71\%) TP53 mutated tumors ( $p=0.098$ ). No differences were found for the type of staining in mutational subtypes (data not shown). In case a cut-off value of $\geq 50 \%$ was used for Dll3 positivity, only for TP53 wildtype tumors compared to TP53 mutated tumors a significantly higher DII3 expression was found (DII3 $\geq 50 \%$ in $8 / 9$ (89\%) vs. 29/58 (50\%), p=0.035) (Supplemental Table S7.B).

\section{DII3 in relation to neuroendocrine marker profile}

In tumors with $\geq 2$ positive NE-markers, Dll3 was expressed significantly more often compared to tumors with 1 positive NE-marker (DIl3+ in 66/82 (81\%) vs. 3/9 (33\%), $\mathrm{p}=0.006)$, and median DII3 $\mathrm{H}$-score was higher in the group with $\geq 2$ positive NE-markers (77.5 (IQ 18-160) vs. 0 (IQ 0-40), p=0.02) (Figure 7.3). Remarkably, 3/3 (100\%) Dll3+ patients with only 1 positive NE-marker had dotlike staining, while in the samples with $\geq 2$ positive NE-markers the fraction of dotlike staining was 11/56 (20\%) (Figure 7.3). Furthermore, an increased median DII3 $\mathrm{H}$-score was associated with an increased staining intensity of Synaptophysin and Chromogranin A, but not with Cd56 (Table 7.2, Supplemental Figure S7.C). Percentage of DII3+ patients was higher in the Ascl1 positive group compared to the Ascl1 negative group (Dll3+ in 35/39 (90\%) vs. 6/12 (50\%), $\mathrm{p}=0.007$ ) (Figure 7.2, Supplemental Table S7.A). Furthermore, Dll3 $\mathrm{H}$-score and Ascl1 Hscore were correlated (Spearman correlation 0.38, $p=0.007$ ) (Supplemental Figure S7.D). 

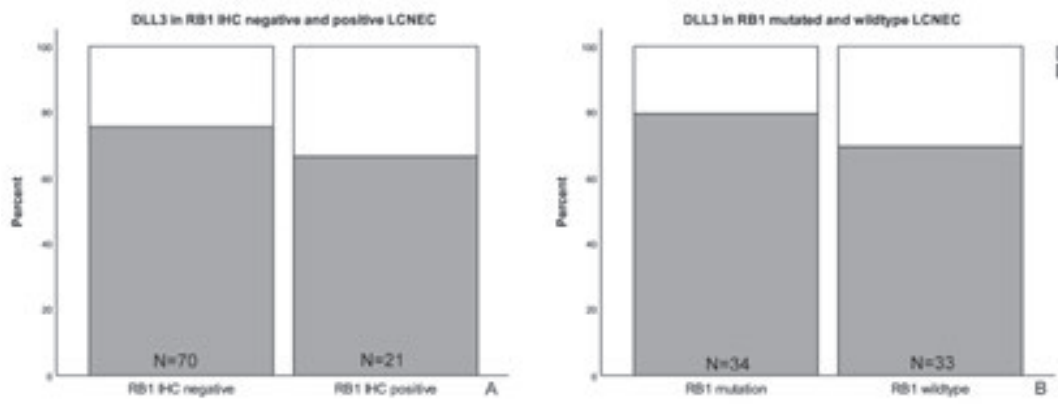

Qous negose
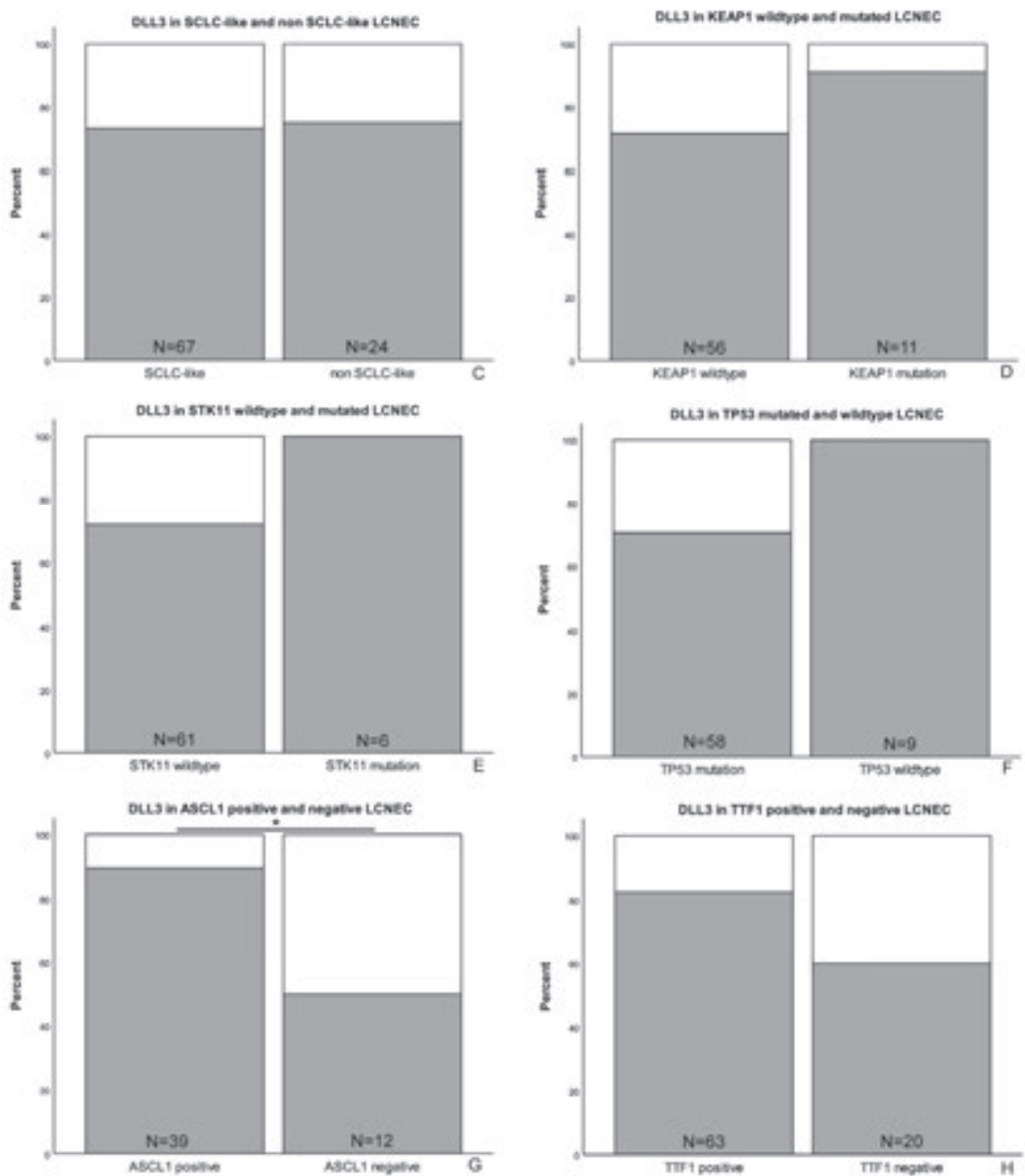

Figure 7.2 DII3 expression in LCNEC: A) pRb IHC negative ( $\mathrm{N}=70)$ \& pRb IHC positive (N=21) B) RB1 mutated $(\mathrm{N}=34)$ \& $R B 1$ wildtype $(\mathrm{N}=33) \mathrm{C}) \mathrm{SCLC}$-like $(\mathrm{N}=67)$ \& non SCLC-like $(\mathrm{N}=24) \mathrm{D})$ KEAP1 wildtype $(\mathrm{N}=56)$ \& KEAP1 mutated $(\mathrm{N}=11)$ E) STK11 wildtype $(\mathrm{N}=61)$ \& STK11 mutated $(\mathrm{N}=6) \mathrm{F})$ TP53 mutated ( $\mathrm{N}=58)$ \& TP53 wildtype $(\mathrm{N}=9) \mathrm{G}$ ) Ascl1 IHC positive ( $\mathrm{N=39}$ ) \& Ascl1 IHC negative $(\mathrm{N}=12) \mathrm{H})$ Ttf1 IHC positive ( $\mathrm{N}=63$ ) \& Ttf1 IHC negative ( $\mathrm{N}=20)$. *Statistically significant (Fisher's Exact Test). 


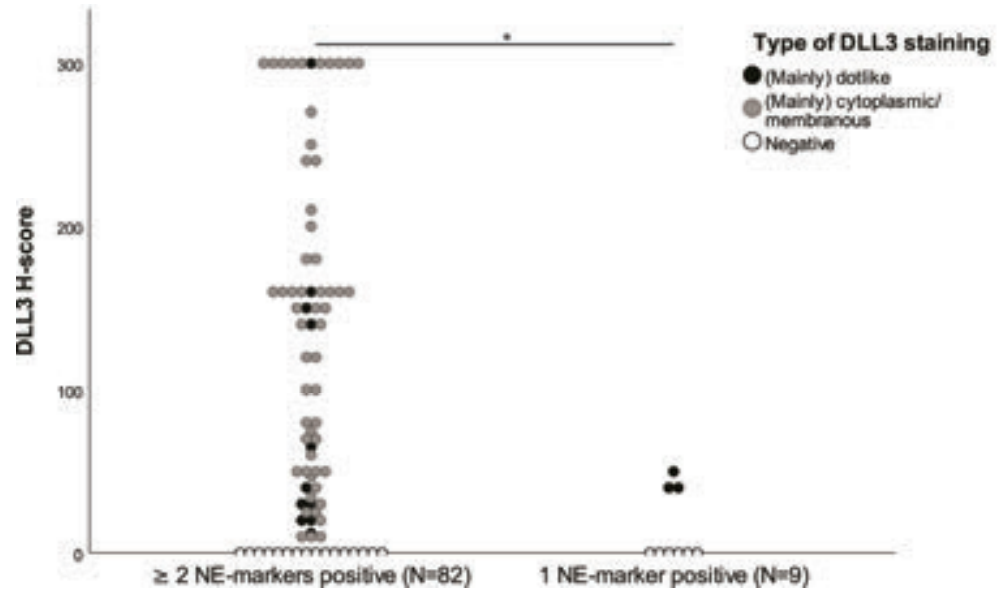

Figure 7.3 DII3 $\mathrm{H}$-score and type of Dll3 staining in tumors with $\geq 2$ positive NE-markers (median $\mathrm{H}$-score 77.5) and 1 positive NE-marker (median $\mathrm{H}$-score $0, \mathrm{p}=0.002$ (Mann-Whitney $\mathrm{U}$ test)). NE-marker $=$ neuroendocrine marker,$-=$ Median $\mathrm{H}$-score

Table 7.2 Correlation between DII3 H-score and staining intensity of neuroendocrine markers.

\begin{tabular}{lcc}
\hline Staining intensity & Median Dll3 H-score (IQ range) & p-value \\
\hline Chromogranin A & & \\
Neg & $40(0-73)$ & $0.015^{*}$ \\
+ & $85(0-185)$ & \\
++ & $65(20-160)$ & $0.001^{*}$ \\
+++ & $160(50-300)$ & \\
Synaptophysin & & \\
Neg & $0(0-20)$ & \\
+ & $80(0-170)$ & $0.55^{*}$ \\
++ & $41(0-135)$ & \\
+++ & $150(43-263)$ & \\
Cd56 & & \\
Neg & $44(8-150)$ & \\
+ & $110(24-243)$ & \\
++ & $50(0-160)$ & \\
+++ & $55(0-160)$ & \\
\hline
\end{tabular}

*Kruskal-Wallis test, IQ range = interquartile range, $+=$ low intensity, $++=$ intermediate intensity, $+++=$ high intensity.

In both subgroups with (almost) $100 \%$ of samples expressing DII3 (STK11/KEAP1 mutated and TP53 wildtype), $100 \%$ of samples were positive for $\geq 2 \mathrm{NE}$-markers. On the contrary, in all 91 patients with all NE-markers performed, $\geq 2$ NE-markers were positive in only 82 samples (90\%). Ascl1 was also highly expressed in the STK11/KEAP1 mutated 
group, whereas an unexpected trend for low Ascl1 expression was seen in the TP53 wildtype group compared to TP53 mutated group (Ascl1+ in 4/8 (50\%) vs. 33/41 (81\%), $\mathrm{p}=0.088$ ).

\section{Discussion}

In this study, we found a high prevalence of DII3 positivity in stage IV LCNEC and demonstrated that DII3 is especially high in STK11 and KEAP1 mutated or TP53 wildtype tumors and in tumors positive for Ascl1 and $\geq 2$ neuroendocrine markers. The prevalence of DII3 expression in LCNEC is comparable to SCLC and might therefore also be a potential therapeutic target in LCNEC.

We demonstrated DII3 expression in $74 \%$ of 94 stage IV LCNEC patients, comparable with the only previous study in LCNEC reporting positive immunohistochemical DII3 staining in $37 / 57$ (65\%) of samples. ${ }^{5}$ So far, no data on type of staining, percentage of positive cells within each sample, or survival related to Dll3 expression was available for LCNEC. In the present study, DII3 was expressed in $\geq 50 \%$ of tumor cells in the majority of samples (54\%). Reported percentages of DII3 expression for SCLC are slightly higher (72-90\%), with the majority of positive samples having a high percentage of tumor cells ( $\geq 50 \%$ ) expressing DII3. ${ }^{5,26-28}$ The majority of LCNEC in our study had cytoplasmic and membranous staining, as was reported before in SCLC. ${ }^{5,28}$ DII3 expression did not correlate with prognosis in this LCNEC cohort. The only study evaluating survival in DII3+ and DII3- SCLC patients without DII3 targeted treatment, demonstrated similar results. $^{28}$

DIl3 expression has been related with mutational status and expression profiles of Ascl1 and Notch1 in LCNEC. George et al. found an $A S C L 1^{\text {High }} / D L L 3^{\text {High }} /$ Notch $^{\text {Low }}$ gene expression profile and high expression levels of neuroendocrine genes (Synaptophysin, Chromogranin A) in LCNEC with TP53 and STK11/KEAP1 mutations. ${ }^{18}$ On the other hand, in LCNEC with TP53 and RB1 mutations, an $A S C L 1^{\text {Low }} / D L L 3^{\text {Low }} /$ Notch $^{\text {High }}$ gene expression profile and lower expression levels of neuroendocrine genes were found. ${ }^{18}$ In accordance with this study, we found all STK11 mutated and 10/11 KEAP1 mutated samples to be immune positive for DII3. Furthermore, a high percentage of those tumors had Ascl1 expression and all had $\geq 2$ NE-markers positive. However, we did not find any relation with RB1 mutation status or pRb IHC staining. In addition, a special subgroup of LCNEC, wildtype for TP53, with an AsCl1 ${ }^{\text {Low }} / \mathrm{DII} 3^{\text {High }}$ profile, was identified. 
Since this study comprises only a limited number of patients in each subgroup, further research is necessary to verify DII3 and Ascl1 expression in these subgroups.

A correlation between Ttf1 and DII3 expression in SCLC was found by Cardnell et al., suggesting that Ttf1 could be used as a surrogate marker for DII3. ${ }^{29}$ We could not confirm this correlation and in our study $28 \%$ of tumors would be misclassified as DII3 IHC positive or negative if Ttf1 would be used as a surrogate marker for DII3.

Recently four subtypes of SCLC were defined by expression of ASCL1, NEUROD1, POU2F3 and YAP1. ${ }^{30}$ Only the first group with ASCL1 expression, the classic SCLC, was found to have high DLL3 expression, whereas the other smaller groups had no or limited expression of DLL3 and ASCL1. ${ }^{30}$ In future research, NEUROD1, POU2F3 and YAP1 could also be tested in LCNEC and correlated to ASCL1 and DLL3 expression.

This study has some limitations. Since it is a retrospective study, not all clinical characteristics (i.e. smoking history) could be obtained. Also, material was not sufficient in all patients to perform NGS, evaluate NE-markers and perform IHC for $p R b$, DII3 and Ascl1. Though clear distinction between dotlike and cytoplasmic staining could be made, discrimination between cytoplasmic staining only and combined cytoplasmic and membranous staining was not possible. Therefore, all cytoplasmic stained samples are considered to have membranous staining as well. Former studies also found a combined cytoplasmic/membranous staining in the majority of tumors. ${ }^{5,28}$ So far, it is not known whether type of staining predicts response to Dll3 targeted therapy. Furthermore, it is not yet known if the cut-off value of $\geq 1 \%$ is clinically relevant or that a higher cut-off value should be chosen. One clinical study found improved outcomes in patients with high DII3 expression ( $\geq 50 \%$ ) compared to low DII3 expression ( $\geq 1-50 \%)$, whereas preliminary results of another study did not find a difference between high $\left(\geq 75 \%\right.$ ) and low ( $\geq 25-75 \%$ ) Dll3 expression. ${ }^{26,31}$ Finally, we used the mouse Dll3 antibody (clone SC16.65) in this study whilst other studies use the rabbit antibody (SP347). So far, no reports are published comparing these two antibodies.

The high percentage of DII3 positive SCLC and LCNEC combined with low or nondetectable DII3 levels in healthy tissue, make DII3 attractive for targeted therapy. ${ }^{5,27,28}$ In normal tissue, DLL3 mRNA is only expressed within the brain and in very low amounts within esophagus and pancreas. ${ }^{5,18}$ The first-in-class drug to target DII3 expressing tumors is an antibody-drug conjugate: rovalpituzumab-tesirine (Rova-T). ${ }^{32}$ After promising results in patient derived xenograft (PDX) mice models and a phase 1 study with Rova-T, several clinical trials were initiated for patients with SCLC and other solid (neuroendocrine) tumors, including LCNEC. $^{5,26}$ Unfortunately, a phase 2 trial (TRINITY) found a response in only a limited number of patients and interim analysis by 
the Independent Data Monitoring Committee of two phase 3 studies (TAHOE and MERU) revealed lack of survival benefit in the Rova-T arm compared to the control arm. $^{33-35}$ Both studies were closed for inclusion and development of Rova-T was halted by Abbvie. ${ }^{34,35}$

Two other approaches of targeting DII3 are a bi-specific T-cell engager (BiTE ${ }^{\circledR}$ ) antibody construct (AMG 757 and DLL3/CD3 ITE) and adoptive chimeric antigen receptor T-cell (CAR-T) therapy (AMG 119). Preclinical studies showed a good safety profile and phase I trials are currently enrolling (NCT03319940 and NCT03392064, respectively). . $^{36,37}$ Hopefully, these new approaches will be more successful than Rova-T in targeting DII3 and treating SCLC and LCNEC.

In this study we demonstrated a high prevalence of cytoplasmic/membranous DII3 positivity in patients with stage IV LCNEC. This high DII3 percentage in LCNEC calls for further study of recently developed DII3 targeting agents such as approaches with BiTE $^{\circledR}$ and CAR-T. 


\section{References}

1. Derks JL, Hendriks LE, Buikhuisen WA, et al. Clinical features of large cell neuroendocrine carcinoma: a population-based overview. Eur Respir J 2016;47:615-624.

2. Asamura $\mathrm{H}$, Kameya $\mathrm{T}$, Matsuno $\mathrm{Y}$, et al. Neuroendocrine neoplasms of the lung: a prognostic spectrum. J Clin Oncol 2006;24:70-76.

3. Rossi G, Cavazza A, Marchioni A, et al. Role of chemotherapy and the receptor tyrosine kinases KIT, PDGFRalpha, PDGFRbeta, and Met in large-cell neuroendocrine carcinoma of the lung. J Clin Oncol 2005;23:8774-8785.

4. Masters GA, Temin S, Azzoli CG, et al. Systemic Therapy for Stage IV Non-Small-Cell Lung Cancer: American Society of Clinical Oncology Clinical Practice Guideline Update. J Clin Oncol 2015;33: 3488-3515.

5. Saunders LR, Bankovich AJ, Anderson WC, et al. A DLL3-targeted antibody-drug conjugate eradicates high-grade pulmonary neuroendocrine tumor-initiating cells in vivo. Sci Transl Med 2015;7:302ra136.

6. Ayyanan A, Civenni G, Ciarloni L, et al. Increased Wnt signaling triggers oncogenic conversion of human breast epithelial cells by a Notch-dependent mechanism. Proc Natl Acad Sci U S A 2006;103:3799-3804.

7. Deng SM, Yan XC, Liang L, et al. The Notch ligand delta-like 3 promotes tumor growth and inhibits Notch signaling in lung cancer cells in mice. Biochem Biophys Res Commun 2017;483:488-494.

8. Chapman G, Sparrow DB, Kremmer E, et al. Notch inhibition by the ligand DELTA-LIKE 3 defines the mechanism of abnormal vertebral segmentation in spondylocostal dysostosis. Hum Mol Genet 2011;20:905-916.

9. Jiang $\mathrm{T}$, Collins BJ, Jin $\mathrm{N}$, et al. Achaete-scute complex homologue 1 regulates tumor-initiating capacity in human small cell lung cancer. Cancer Res 2009;69:845-854.

10. Henke RM, Meredith DM, Borromeo MD, et al. Ascl1 and Neurog2 form novel complexes and regulate Delta-like3 (DII3) expression in the neural tube. Dev Biol 2009;328:529-540.

11. Nakakura EK, Sriuranpong VR, Kunnimalaiyaan M, et al. Regulation of neuroendocrine differentiation in gastrointestinal carcinoid tumor cells by notch signaling. J Clin Endocrinol Metab 2005;90:4350-4356.

12. Sriuranpong V, Borges $\mathrm{MW}$, Strock $\mathrm{CL}$, et al. Notch signaling induces rapid degradation of achaete-scute homolog 1. Mol Cell Biol 2002;22:3129-3139.

13. Maemura K, Yoshikawa H, Yokoyama K, et al. Delta-like 3 is silenced by methylation and induces apoptosis in human hepatocellular carcinoma. Int J Oncol 2013;42:817-822.

14. Espinoza I, Miele L. Notch inhibitors for cancer treatment. Pharmacol Ther 2013;139:95-110.

15. Kunnimalaiyaan $\mathrm{M}$, Chen $\mathrm{H}$. Tumor suppressor role of Notch-1 signaling in neuroendocrine tumors. Oncologist 2007;12:535-542.

16. Linnoila RI, Zhao B, DeMayo JL, et al. Constitutive achaete-scute homologue-1 promotes airway dysplasia and lung neuroendocrine tumors in transgenic mice. Cancer Res 2000;60:4005-4009.

17. Rekhtman N, Pietanza MC, Hellmann MD, et al. Next-Generation Sequencing of Pulmonary Large Cell Neuroendocrine Carcinoma Reveals Small Cell Carcinoma-like and Non-Small Cell Carcinoma-like Subsets. Clin Cancer Res 2016;22:3618-3629.

18. George J, Walter V, Peifer $\mathrm{M}$, et al. Integrative genomic profiling of large-cell neuroendocrine carcinomas reveals distinct subtypes of high-grade neuroendocrine lung tumors. Nat Commun 2018;9:1048.

19. Derks JL, Leblay N, Lantuejoul S, et al. New Insights into the Molecular Characteristics of Pulmonary Carcinoids and Large Cell Neuroendocrine Carcinomas, and the Impact on Their Clinical Management. J Thorac Oncol 2018;13:752-766.

20. Derks JL, Leblay N, Thunnissen E, et al. Molecular Subtypes of Pulmonary Large-cell Neuroendocrine Carcinoma Predict Chemotherapy Treatment Outcome. Clin Cancer Res 2018;24:33-42.

21. Derks JL, van Suylen RJ, Thunnissen E, et al. A Population-Based Analysis of Application of WHO Nomenclature in Pathology Reports of Pulmonary Neuroendocrine Tumors. J Thorac Oncol 2016;11:593-602. 
22. Casparie M, Tiebosch AT, Burger G, et al. Pathology databanking and biobanking in The Netherlands, a central role for PALGA, the nationwide histopathology and cytopathology data network and archive. Cell Oncol 2007;29:19-24.

23. Travis WD, Brambilla E, Burke AP, et al. WHO Classification of Tumours of the Lung, Pleura, Thymus and Heart Lyon, France: International Agency for Research on Cancer; 2015.

24. Derks JL, van Suylen RJ, Thunnissen E, et al. Chemotherapy for pulmonary large cell neuroendocrine carcinomas: does the regimen matter? Eur Respir J 2017;49.

25. Derks JL, Dingemans AC, van Suylen RJ, et al. Is the sum of positive neuroendocrine immunohistochemical stains useful for diagnosis of large cell neuroendocrine carcinoma (LCNEC) on biopsy specimens? Histopathology 2019;74:555-566.

26. Rudin CM, Pietanza MC, Bauer TM, et al. Rovalpituzumab tesirine, a DLL3-targeted antibody-drug conjugate, in recurrent small-cell lung cancer: a first-in-human, first-in-class, open-label, phase 1 study. Lancet Oncol 2017;18:42-51.

27. Saito M, Saito K, Shiraishi K, et al. Identification of candidate responders for anti-PD-L1/PD-1 immunotherapy, Rova-T therapy, or EZH2 inhibitory therapy in small-cell lung cancer. Mol Clin Oncol 2018;8:310-314.

28. Tanaka K, Isse K, Fujihira T, et al. Prevalence of Delta-like protein 3 expression in patients with small cell lung cancer. Lung Cancer 2018;115:116-120.

29. Cardnell RJ, Li L, Sen T, et al. Protein expression of TTF1 and cMYC define distinct molecular subgroups of small cell lung cancer with unique vulnerabilities to aurora kinase inhibition, DLL3 targeting, and other targeted therapies. Oncotarget 2017;8:73419-73432.

30. Rudin CM, Poirier JT, Byers LA, et al. Molecular subtypes of small cell lung cancer: a synthesis of human and mouse model data. Nat Rev Cancer 2019;19:289-297.

31. Carbone D, Morgensztern D, Moulec SL, et al. Efficacy and safety of rovalpituzumab tesirine in patients with DLL3-expressing, $\geq 3$ rd line small cell lung cancer: Results from the phase 2 TRINITY study. ASCO Annual Meeting; Abstract \#8507 2018.

32. Tiberghien AC, Levy JN, Masterson LA, et al. Design and Synthesis of Tesirine, a Clinical Antibody-Drug Conjugate Pyrrolobenzodiazepine Dimer Payload. ACS Med Chem Lett 2016;7:983-987.

33. Morgensztern D, Besse B, Greillier L, et al. Efficacy and Safety of Rovalpituzumab Tesirine in Third-Line and Beyond Patients with DLL3-Expressing, Relapsed/Refractory Small Cell Lung Cancer: Results From the Phase II TRINITY Study. Clin Cancer Res. 2019;25(23):6958-6966.

34. Phase 3 Trial of Rova-T as Second-line Therapy for Advanced Small-Cell Lung Cancer (TAHOE Study) Halted [press release]. AbbVie, North Chicago; 2018.

35. AbbVie Discontinues Rovalpituzumab Tesirine (Rova-T) Research and Development Program [press release]. Abbvie, North Chicago; 2019.

36. Giffin M, Cooke K, Lobenhofer E, et al. Targeting DLL3 with AMG 757, a BiTE ${ }^{\circledR}$ Antibody Construct, and AMG 119, a CAR-T, for the Treatment of SCLC. J Thorac Oncol 2018;13:Supplement.

37. Hipp S, Voynov V, Drobits-Handl B, et al. A novel T-cell engaging bispecific antibody induces specific and efficacious lysis of small cell lung cancer cells in vitro and potent T cell re-directed anti-tumor activity in vivo (Abstract \#549). AACR. Atlanta: 2019;Part A: Abstracts 1-2748. 


\section{Supplemental material}

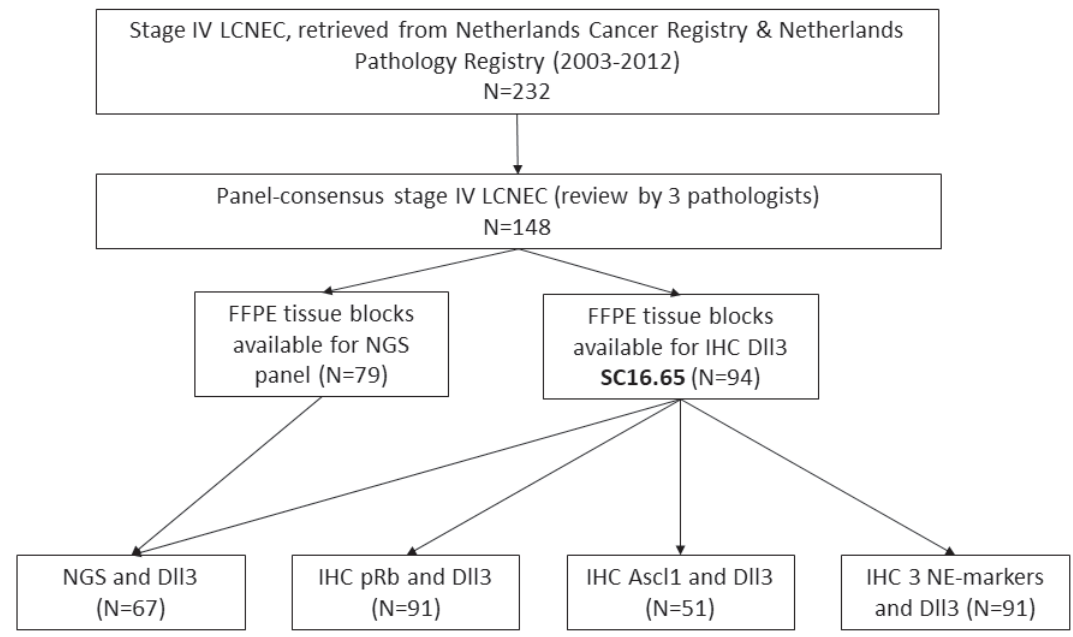

Figure S7.A Selection of patients for panel-consensus review, mutational analysis and IHC for DII3, Ascl1 and $\mathrm{pRb}$. Abbreviations: $\mathrm{N}=$ number, LCNEC = large cell neuroendocrine carcinoma, $\mathrm{FFPE}=$ formalin-fixed paraffin embedded, $\mathrm{NGS}=$ next generation sequencing, $\mathrm{IHC}=$ immunohistochemistry, NE-markers = neuroendocrine markers (Synaptophysin, Chromogranin A, Cd56).

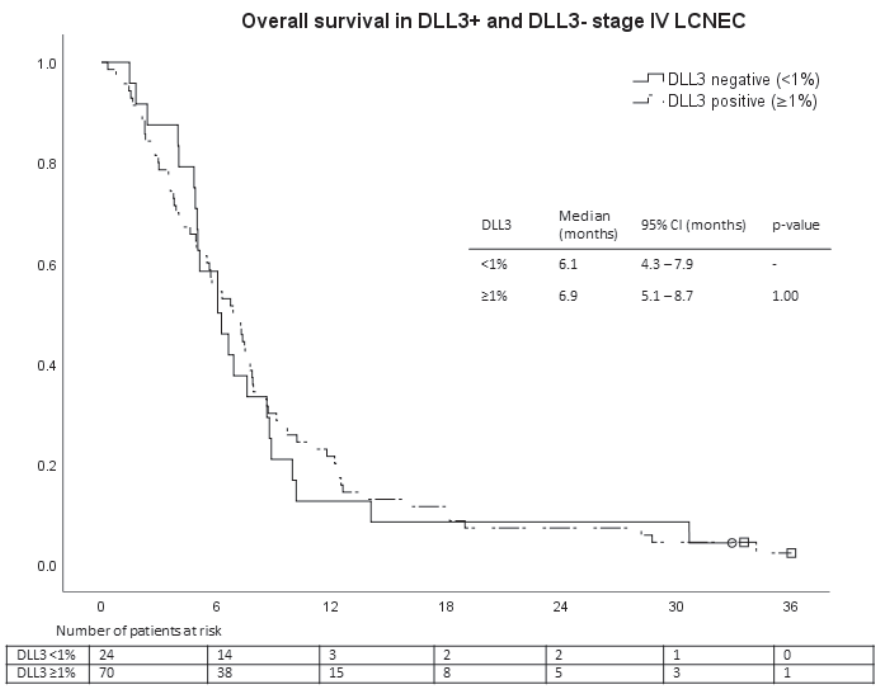

Figure S7.B Overall survival in patients with DII3 immunohistochemistry negative $(<1 \%)$ and DII3 positive $(\geq 1 \%)$ LCNEC. 

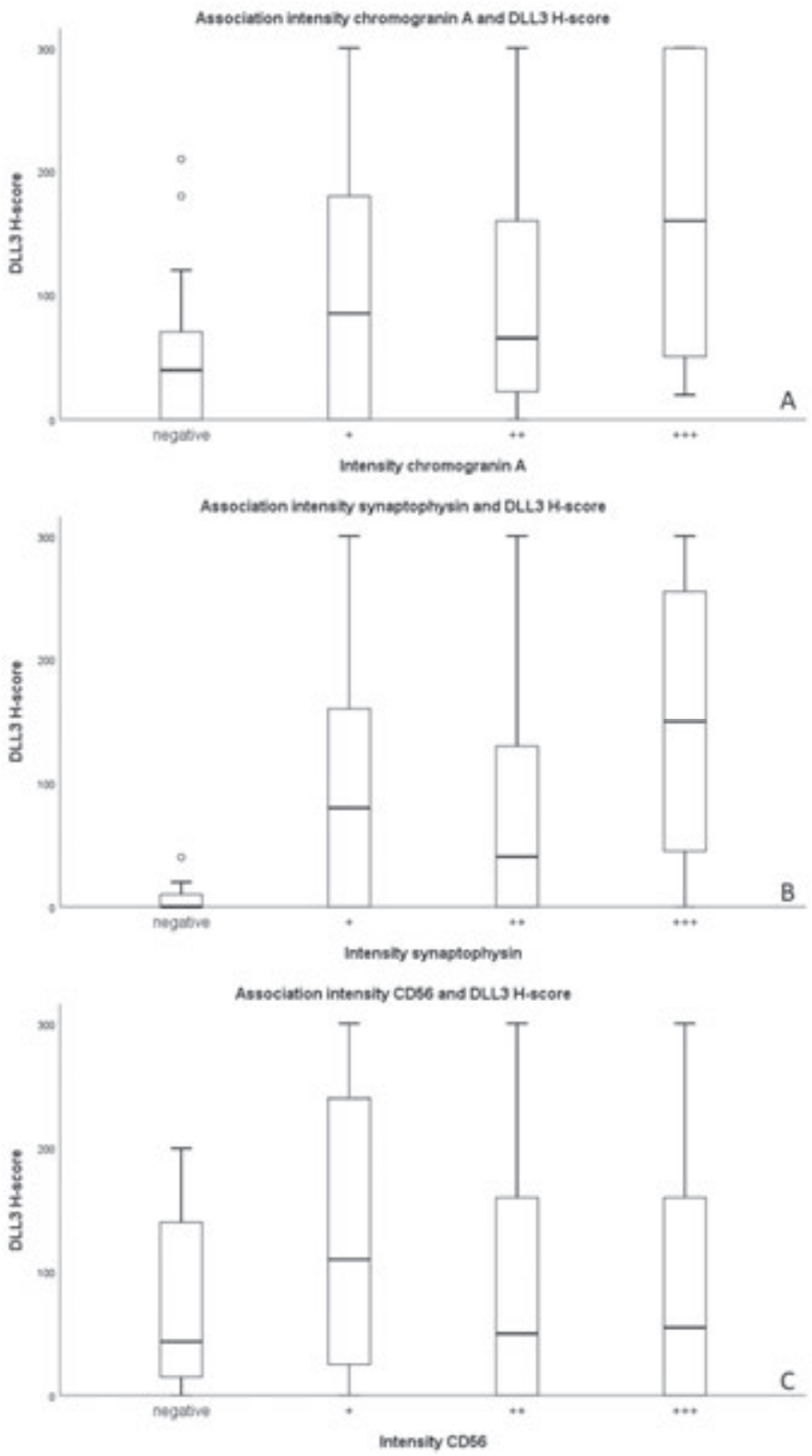

Figure S7.C Median DII3 H-score and interquartile ranges in patients with negative, low $(+)$, intermediate $(++)$ or high $(+++)$ staining for neuroendocrine markers. A) Chromogranin A, B) Synaptophysin, C) $\mathrm{Cd} 56$. 


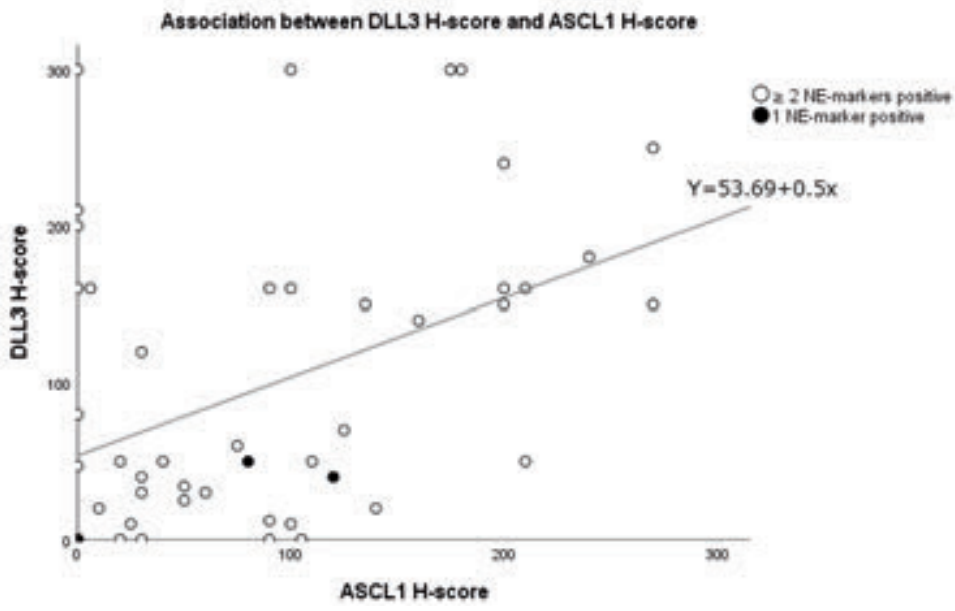

Figure S7.D Correlation between DII3 H-score and Ascl1 H-score with Spearman correlation $0.38(p<0.007)$.

Table S7.A DII3 expression ( $\geq 1 \%$ and $<1 \%$ ) in mutational subtypes of stage IV LCNEC.

\begin{tabular}{|c|c|c|c|}
\hline & DII3 $\geq 1 \%$ & DII3 <1\% & p-value \\
\hline pRb Expressing & $14(67 \%)$ & $7(33 \%)$ & $0.41^{*}$ \\
\hline pRb Non-expressing & $53(76 \%)$ & $17(24 \%)$ & \\
\hline RB1wt & $23(70 \%)$ & $10(30 \%)$ & $0.36^{*}$ \\
\hline RB1mt & $27(79 \%)$ & $7(21 \%)$ & \\
\hline SCLC-like LCNEC & $49(73 \%)$ & $18(37 \%)$ & $0.86^{*}$ \\
\hline Non SCLC-like LCNEC & $18(75 \%)$ & $6(25 \%)$ & \\
\hline TP53wt & $9(100 \%)$ & $0(0 \%)$ & $0.098 * *$ \\
\hline TP53mt & $41(71 \%)$ & $17(29 \%)$ & \\
\hline STK11wt & $44(72 \%)$ & $17(28 \%)$ & $0.33^{* *}$ \\
\hline STK11mt & $6(100 \%)$ & $0(0 \%)$ & \\
\hline KEAP1wt & $40(71 \%)$ & $16(29 \%)$ & $0.27^{* *}$ \\
\hline KEAP1mt & $10(91 \%)$ & 1 (9\%) & \\
\hline \multicolumn{4}{|l|}{ Ascl1 ( $N=51)$} \\
\hline Expressing & $35(90 \%)$ & $4(10 \%)$ & $0.007 * *$ \\
\hline Non-expressing & $6(50 \%)$ & $6(50 \%)$ & \\
\hline \multicolumn{4}{|l|}{ Ttf1 (N=83) } \\
\hline Expressing & $52(83 \%)$ & $11(18 \%)$ & $0.063^{* *}$ \\
\hline Non-expressing & $12(60 \%)$ & $8(40 \%)$ & \\
\hline
\end{tabular}

* Chi-square; ** Fisher's Exact Test. 
Table S7.B DII3 expression ( $\geq 50 \%$ and $<50 \%$ ) in mutational subtypes of stage IV LCNEC.

\begin{tabular}{|c|c|c|c|}
\hline & DII3 $\geq 50 \%$ & DIl3 $<50 \%$ & p-value \\
\hline pRb Expressing & $10(48 \%)$ & $11(52 \%)$ & \multirow{2}{*}{$0.51^{*}$} \\
\hline pRb Non-expressing & 39 (56\%) & $31(44 \%)$ & \\
\hline RB1wt & $19(58 \%)$ & $14(42 \%)$ & \multirow{2}{*}{$0.70^{*}$} \\
\hline RB1mt & $18(53 \%)$ & $16(47 \%)$ & \\
\hline SCLC-like LCNEC & $36(54 \%)$ & $31(46 \%)$ & \multirow{2}{*}{$0.97^{*}$} \\
\hline Non SCLC-like LCNEC & $13(54 \%)$ & $11(46 \%)$ & \\
\hline TP53wt & $8(89 \%)$ & $1(11 \%)$ & \multirow{2}{*}{$0.035^{* *}$} \\
\hline TP53mt & $29(50 \%)$ & $29(50 \%$ & \\
\hline STK11wt & $34(56 \%)$ & $27(44 \%)$ & \multirow{2}{*}{$1.00 * *$} \\
\hline STK11mt & $3(50 \%)$ & $3(50 \%)$ & \\
\hline KEAP1wt & $30(54 \%)$ & $26(46 \%)$ & \multirow{2}{*}{$0.74 * *$} \\
\hline KEAP1mt & $7(64 \%)$ & $5(36 \%)$ & \\
\hline \multicolumn{4}{|l|}{ Ascl1 ( $N=51)$} \\
\hline Expressing & $24(62 \%)$ & $15(39 \%)$ & \multirow{2}{*}{$0.22 *$} \\
\hline Non-expressing & $5(42 \%)$ & $7(58 \%)$ & \\
\hline \multicolumn{4}{|l|}{ Ttf1 (N=83) } \\
\hline Expressing & $40(64 \%)$ & $23(37 \%)$ & \multirow{2}{*}{$0.064 *$} \\
\hline Non-expressing & $8(40 \%)$ & $12(60 \%)$ & \\
\hline
\end{tabular}

* Chi-square; ** Fisher's Exact Test. 



\section{Chapter 8}

\section{Prevalence and prognostic value of $\mathrm{Pd}-\mathrm{I} 1$ expression in molecular subtypes of metastatic large cell neuroendocrine carcinoma (LCNEC)}

B.C.M. Hermans, J.L. Derks, E. Thunnissen, R. J. van Suylen, M.A. den Bakker, H.J.M. Groen, E.F. Smit, R.A. Damhuis, E.C. van den Broek, PALGA-group, C.M. Stallinga, G.M. Roemen, E.J.M. Speel*, A-M.C. Dingemans* * contributed equally to this work 


\section{Abstract}

\section{Background}

Pulmonary large cell neuroendocrine carcinoma (LCNEC) is a rare tumor with high mutational burden. Two subtypes of LCNEC are recognized, the co-mutated TP53 and RB1 group and the TP53 and STK11/KEAP1 group. We investigated Pd-I1 and Cd8 expression in a well characterized stage IV LCNEC cohort and compared expression in the two subtypes.

\section{Methods}

Immunohistochemical (IHC) analysis for $\mathrm{Pd}-\mathrm{I} 1$ and $\mathrm{Cd} 8$ was performed on pathological reviewed pretreatment tumor samples for 148 stage IV LCNEC. Data about targeted next generation sequencing (tNGS) (TP53, RB1, STK11, KEAP1) and IHC for pRb were available for most tumors. IHC staining for Pd-I1 (DAKO 28-8) was performed and scored positive if tumors showed $\geq 1 \%$ membranous staining. Cd8 was scored for intratumor T-cells and stromal cells.

\section{Results}

Pd-I1 IHC expression data could be generated in 98/148 confirmed LCNEC samples along with $\mathrm{pRb}$ IHC ( $\mathrm{n}=97)$ of which 77 passed quality control for tNGS. Pd-I1 expression was positive in $16 / 98$ cases (16\%); 5 (5\%) with $\geq 50 \%$. Pd-I1 expression was equal in $R B 1$ mutated and RB1 wildtype tumors. None of STK11 mutated tumors $(\mathrm{n}=7)$ expressed $\mathrm{Pd}$ I1. Pd-I1 expression was correlated with superior overall survival (OS), hazard ratio 0.55 ((95\% Confidence Interval 0.31-0.96), $\mathrm{p}=0.038)$. Intra-tumor $\mathrm{Cd} 8$ was associated with Pd-I1 expression $(p=0.021)$ and stromal and intra-tumor $\mathrm{Cd} 8$ were correlated with improved OS ( $p=0.037$ and $p=0.026$ respectively).

\section{Conclusion}

Pd-I1 expression was positive in $16 \%$ of stage IV LCNEC tumors. This was independent of molecular subtype but associated with Cd8 expression. In LCNEC patients with Pd-I1 and/or Cd8 expression superior OS was observed. 


\section{Introduction}

Large cell neuroendocrine carcinoma (LCNEC) of the lung is an uncommon tumor, representing $1-3 \%$ of all types of lung cancer. ${ }^{1,2}$ Although LCNEC shows hallmarks of non-small cell lung cancer (NSCLC), prognosis seems to be similar to small cell lung cancer (SCLC) with poor survival rates. ${ }^{1,3,4}$ In LCNEC neuroendocrine morphology is required, and if present confirmation of neuroendocrine differentiation by immunohistochemistry (IHC) is necessary in the WHO 2015 classification. ${ }^{5}$ Next generation sequencing (NGS) studies have identified two exclusive molecular subtypes of LCNEC. A subtype with inactivation of TP53 and STK11 and/or KEAP1 genes, a second subtype with mutation of TP53 and $R B 1$ (a hallmark of SCLC). ${ }^{6-8}$ These subtypes may be relevant for prognosis and response to therapy.

For stage IV LCNEC tumors, palliative chemotherapy is the treatment of choice. However, owing to the rarity of the tumor, no large randomized controlled trials concerning the most appropriate chemotherapy have been performed and currently both SCLC and NSCLC chemotherapy regimens are deemed appropriate. In a recent retrospective study, we showed relevance for the molecular subtyping. The study revealed that patients with LCNEC and wildtype RB1 (NSCLC-like) had a longer overall survival (OS) when treated with NSCLC regime (platinum doublet with gemcitabine, docetaxel or paclitaxel) compared to SCLC regime (platinum-etoposide) or NSCLC regime containing pemetrexed. In contrast, no difference was observed in LCNEC cases with $R B 1$ mutation (SCLC-like). ${ }^{9}$

In NSCLC, Pd-I1 expression has been reported in up to $60 \%$ of tumors and Pd-1/Pd-I1 targeted therapy with or without chemotherapy is standard of care in patients without EGFR or ALK mutation. ${ }^{10-15}$ Approximately $30 \%$ of SCLC tumors are Pd-I1 positive. However, due to insufficient data Pd-1/Pd-I1 targeted therapy for SCLC is so far only recommended in the National Comprehensive Cancer Network (USA) guideline as combination therapy. ${ }^{16-18}$ Scarce data exist about Pd-I1 expression in LCNEC, with prevalence of $\mathrm{Pd}-\mathrm{I1}$ positivity reported in $9-32 \%$ of patients and conflicting results with respect to the prognostic relevance of Pd-11. ${ }^{19-25}$ Importantly, the majority of LCNEC studies evaluated surgically resected cases with non-metastatic disease whereas data on Pd-I1 expression in metastatic (stage IV) disease is lacking. However, immunotherapy is of special interest in LCNEC since LCNEC has a high mutational burden (up to 11 mutations per $\mathrm{Mb}$ ), and this may be related to response to immunotherapy. ${ }^{6,7,9,26-28}$ 
In this study we evaluated the prevalence of $\mathrm{Pd}-\mathrm{I} 1$ expression in a large cohort of patients with well characterized and molecular profiled stage IV LCNEC. We furthermore investigated $\mathrm{Pd}-\mathrm{I} 1$ expression related to different mutational profiles (i.e. RB1 mutation vs. STK11/KEAP1 mutation) and to Cd8 positive cells as a marker of immune system activity. We also studied the prognostic value of $\mathrm{Pd}-\mathrm{I} 1$ and $\mathrm{Cd} 8$ expression in these LCNEC patients.

\section{Materials and methods}

\section{Patient and tissue selection}

For this retrospective population-based study all data were retrieved from the Netherlands Cancer Registry and Netherlands Pathology Registry (PALGA) as described previously. ${ }^{29,30}$ For all 232 stage IV LCNEC, diagnosed between 2003 and 2012 in the Netherlands on a pre-treatment sample, panel consensus pathology revision was performed as described earlier by three pathologists (ET, MdB \& RvS). ${ }^{31}$ Samples were scored for neuroendocrine morphology (organoid nesting, palisading, rosettes or trabeculae), mitotic index, necrosis and neuroendocrine differentiation (positive immunohistochemistry (IHC) for at least one neuroendocrine marker). Diagnosis was confirmed in patients meeting the WHO-criteria. ${ }^{5}$ An exception was made when strict WHO-criteria were not met, but the pathologists found it highly likely that LCNEC was the correct diagnosis, as described earlier. ${ }^{31,32}$ In patients with panel consensus confirmed LCNEC ( $n=148)$, targeted NGS was performed on tumor tissue from available FFPE tissue blocks for the genes RB1, KEAP1, STK11 and TP53. Furthermore, IHC staining was executed for $\mathrm{pRb}$ protein. Data concerning age, gender, OS, chemotherapy details and date of death or last day of follow-up were available and updated until $2015 .^{9}$

The study protocol was approved by the medical ethical committee of the Maastricht University Medical Centre (METC azM/UM 14-4-043). The study is performed according to the Dutch "Federa, Human Tissue and Medical Research: Code of conduct for responsible use (2011)" regulations not requiring patient informed consent.

\section{Immunohistochemistry}

\section{$P d-/ 1$}

IHC staining for Pd-I1 was performed with the monoclonal rabbit anti-Pd-I1 clone $28-8$ using the DAKO Autostainer Link 48 system with the Pd-I1 IHC 28-8 pharmDx kit (DAKO, 
Agilent, USA) according to recommended protocols. Low $\mathrm{pH}$ target retrieval solution and Rabbit linker were used. Evaluation of the percentage tumor cells with partial or complete membranous staining was performed by EJS and $\mathrm{BH}$. Tumor proportion score (TPS) was defined as the percentage of tumor cells with complete or partial membranous staining at any intensity. A TPS $\geq 1 \%$ was considered as positive. A distinction was made between Pd-I1+ high ( $\geq 50 \%)$ and Pd-I1+ low (1-49\%).

\section{Cd8}

DAKO C8/144B antibody was used for Cd8 immunohistochemistry to stain T-cells on the DAKO autostainer link 48 system, high $\mathrm{pH}$ target retrieval was used. Samples were evaluated by two investigators (EJS and $\mathrm{BH}$ ). $\mathrm{Cd} 8$ density in tumor-associated stromal cells was arbitrary scored as negative, weakly positive, moderately positive or strongly positive. Cd8 positive cells in the tumor were scored as negative, $\leq 1 \%$ or $>1 \%$. When Cd8 invasion was scored $>1 \%$ counting of $\mathrm{Cd} 8$ positive cells was performed by evaluating three representative parts of the tumor with 200x amplification. Mean number of $\mathrm{Cd} 8$ positive cells per $\mathrm{mm}^{2}$ was calculated.

\section{Mutational analysis}

Targeted next generation sequencing had already been performed as described previously, covering the exons of TP53, RB1, STK11 and KEAP1. ${ }^{9}$ Immunohistochemistry was performed for $\mathrm{pRb}$ with mouse antibody 13A10, with tonsillar tissue and tumor stromal cells as positive and negative controls, as reported before. ${ }^{9}$

\section{Statistics}

All analyses were performed using SPSS (version 25 for Windows, Armonk, NY: IBM Corp.). Patient characteristics were evaluated with descriptive statistics. Correlation of Pd-I1 expression with age, gender, mutational status (TP53, RB1, STK11 and KEAP1) and $\mathrm{IHC}$ staining for $\mathrm{pRb}$ and $\mathrm{Cd} 8$ was investigated using the chi-square test. Median OS was evaluated by Kaplan Meier analysis and differences in survival were tested for significance with Log-Rank test ( $\mathrm{p}<0.05$ was considered significant) for IHC for Pd-I1, Cd8 in the tumor, Cd8 in stromal cells and $\mathrm{pRb}$, and for mutation status of RB1, STK11, KEAP1 and TP53. Multivariable cox-regression analysis included all factors with a significant impact ( $\mathrm{Pd}-\mathrm{I} 1$ and $\mathrm{Cd} 8$ in stromal cells), completed with the known prognostic factors age and gender. Results are presented as hazard ratios (HR) with $95 \%$ confidence intervals $(\mathrm{Cl})$. 


\section{Results}

\section{Patient characteristics}

After selection of cases with sufficient tumor material for IHC staining, 98 pathology confirmed LCNECs treated with chemotherapy were stained for $\mathrm{Pd}-\mathrm{I} 1$, and 93/98 for Cd8 (Table 8.1). The vast majority of those patients (85/98 for Pd-I1 and 80/93 for Cd8 respectively) fulfilled WHO criteria (Supplemental Table S8.A). For 97/98 cases pRb IHC data were available and for 77/98 cases targeted NGS data for TP53, RB1, STK11 and KEAP1 (Supplemental Figure S8.A). Median age at diagnosis of the 98 patients was 64 years (range 34-82 years). A total of $61 \%$ patients were male (Table 8.1). Chemotherapy included SCLC regimen (including a platinum component and etoposide) in $35 \%$ of patients, NSCLC regimen (including a platinum component with either gemcitabine, docetaxel or paclitaxel) in $44 \%$, platinum-pemetrexed in $12 \%$ and $9 \%$ unspecified, respectively.

Table 8.1 Expression of Pd-I1 in LCNEC, patient characteristics and survival.

\begin{tabular}{lccc}
\hline & Pd-l1+ & Pd-l1- & p-value \\
\hline LCNEC (n=98) & $16(16 \%)$ & $82(84 \%)$ & - \\
$1-<50 \%$ & $11(11 \%)$ & - & - \\
$\geq 50 \%$ & $5(5 \%)$ & - & - \\
Age (median, range) & $63(37-74)$ & $64(34-82)$ & $0.837^{\#}$ \\
Gender & & & \\
$\quad$ Male & $10(63 \%)$ & $50(61 \%)$ & $0.909^{\#}$ \\
Female & $6(38 \%)$ & $32(39 \%)$ & \\
OS in months (95\% Cl) & $8.9(4.1-13.6)$ & $6.6(5.6-7.6)$ & HR $0.55(0.31-0.96)$ \\
& & & p=0.038 \\
\hline
\end{tabular}

${ }^{\#}$ Chi-square test (for age group $\leq 65$ and $>65$ ). ${ }^{*}$ Cox-regression including age and gender. Abbreviations: OS = overall survival; $95 \% \mathrm{Cl}=95 \%$ confidence interval; $\mathrm{HR}=$ hazard ratio.

\section{Pd-I1 expression}

Membranous staining of tumor cells for Pd-I1 ( $\geq 1 \%$ ) was observed in 16/98 (16\%) LCNEC, staining was negative in $82 / 98$ (84\%) (Table 8.1). Positive staining included $n=5$ (5\%) LCNEC cases with $\geq 50 \%$ staining and $n=11$ (11\%) with $1-49 \%$ staining (Figure 8.1 ). Outcome of Pd-11 expression was not associated with age or gender (Table 8.1). Subgroup analysis of the 85 patients with strict WHO-diagnosis was comparable to the results of the full cohort (Supplemental Table S8.A). 


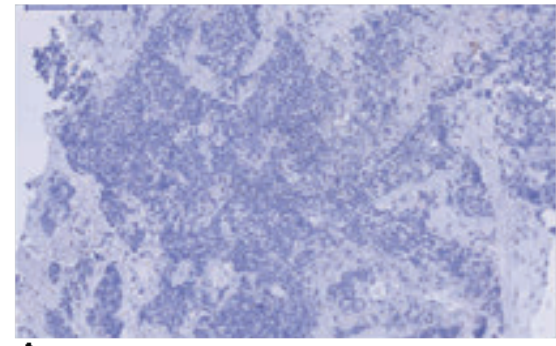

A
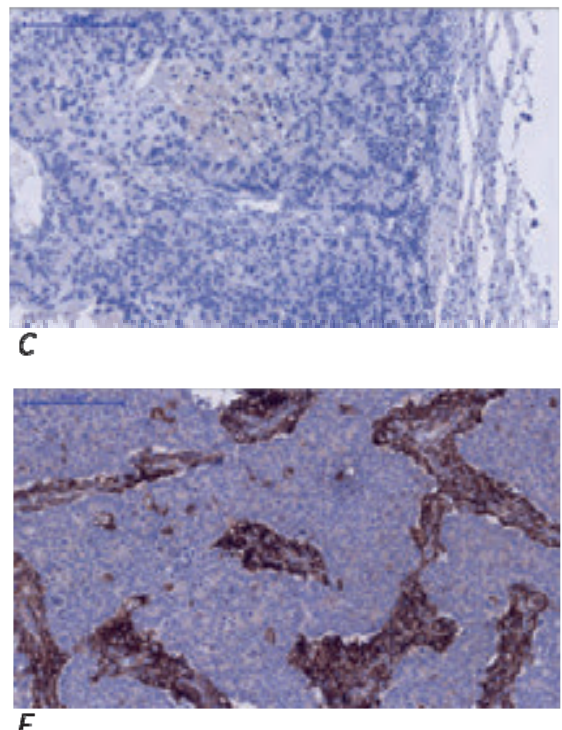

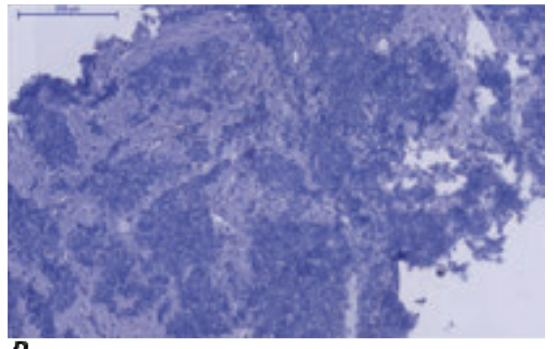

B
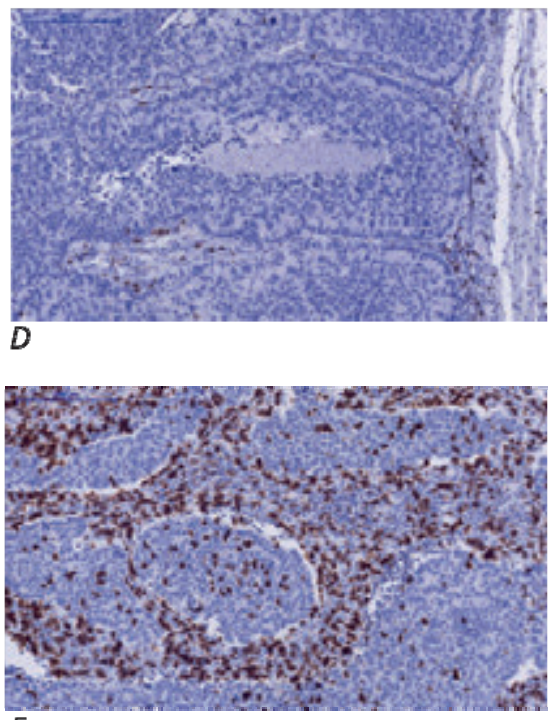

$F$

Figure 8.1 Pathological slide overview of three patients with Pd-11 28-8 and Cd8 staining. A) Patient 1; PdI1 negative. B) Patient 1; Cd8 negative. C) Patient 2; Pd-I1 negative. D) Patient 2; Cd8 stromal cells positive (weak), tumor cells negative. E) Patient 3; Pd-I1 positive. F) Patient 3; Cd8 tumor cells positive and stromal cells strongly positive.

\section{Pd-I1 expression in molecular subgroups of LCNEC}

The frequency of tumors positive for Pd-I1 expression was equal in RB1 mutated (SCLClike) and RB1 wildtype (NSCLC-like) LCNEC ( $\mathrm{n}=6(17 \%)$ vs. $\mathrm{n}=6(15 \%)$, respectively, $\mathrm{p}=0.842)$. All seven STK11 mutated tumors were $P d-I 1$ negative $(p=0.229)$. A higher frequency of Pd-I1 positive LCNEC was observed in TP53 wildtype tumors (TP53 wildtype $n=5(36 \%), T P 53$ mutated $n=8(12 \%), p=0.043)$ (Figure 8.2 , Supplemental Table S8.B). Results were comparable for the subgroup of patients meeting WHO criteria (Supplemental Table S8.C). 

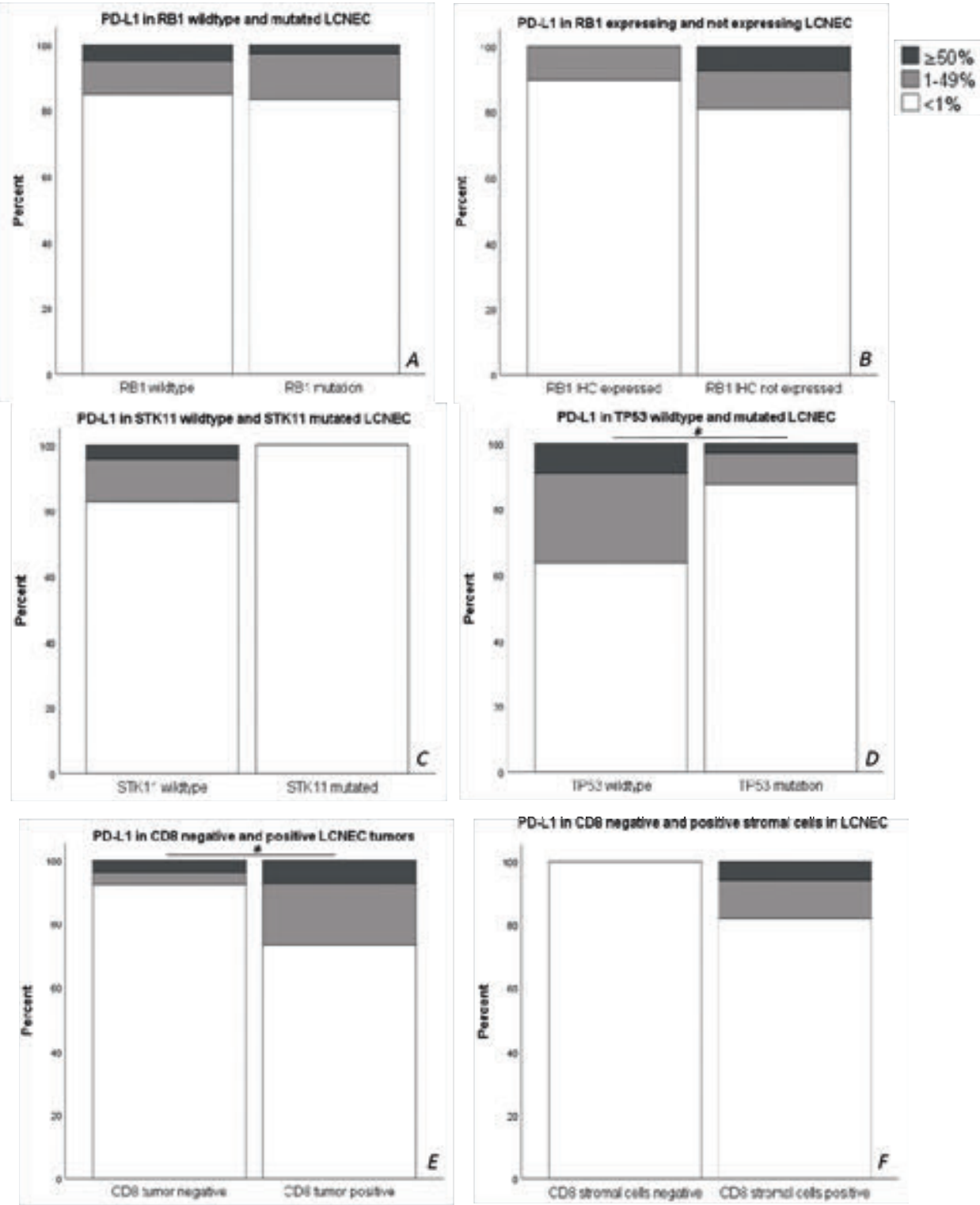

Figure 8.2 Pd-11 expression in LCNEC patients: A) RB1 wildtype $(\mathrm{N}=40)$ \& mutated $(\mathrm{N}=36) \quad \mathrm{B}) \mathrm{pRb}$ expressing $(\mathrm{N}=29)$ \& non-expressing $(\mathrm{N}=68)$ C) STK11 wildtype $(\mathrm{N}=69)$ \& mutated $(\mathrm{N}=7)$ D) TP53 wildtype $(\mathrm{N}=11)$ \& mutated $(\mathrm{N}=65)$ E) Cd8 non-expressing ( $N=52)$ \& expressing $(\mathrm{N}=41)$ in T-cells in tumor $\mathrm{F}) \mathrm{Cd} 8$ non-expressing $(\mathrm{N}=10)$ \& expressing $(\mathrm{N}=83)$ in stromal cells. 


\section{$\mathrm{Cd} 8$}

Any intra-tumor Cd8 staining was observed in 41/93 (44\%) LCNEC and Cd8 staining of $>1 \%$ was observed in 15/93 (16\%) of LCNEC (Figure 8.1, Supplemental Table S8.D). In LCNEC with Cd8 count estimated at $>1 \%$, Cd8 counting exhibited a mean density of 142 cells $/ \mathrm{mm}^{2}$ (minimum 15 cells $/ \mathrm{mm}^{2}$, maximum 376 cells $/ \mathrm{mm}^{2}$ ) (Supplemental Table S8.E). Analysis of stromal tissue showed staining in 83/93 (89\%) LCNEC; including $n=57$ (61\%) weak positive, $n=7$ (8\%) moderate positive, $n=19(20 \%)$ strong positive (Supplemental Table S8.D). Intra-tumor Cd8 expression and Cd8 expression in tumoradjacent stroma was associated, with $98 \%(n=40)$ of samples positive in the tumor also being positive in stromal cells $(p=0.039)$. Expression of $\mathrm{Pd}-\mathrm{I} 1$ was associated with the presence of intra-tumor $\mathrm{Cd} 8(\mathrm{p}=0.013)$ (Figure 8.2, Supplemental Table S8.B). Cd8 expression in both intra-tumor and stroma was comparable in RB1 mutated (15/36, $42 \%)$ and $R B 1$ wildtype $(20 / 36,56 \%)$ LCNEC ( $p=0.238)$. All seven STK11 mutated tumors had $\leq 1 \%$ intra-tumor Cd8 staining $(p=0.332)$. Subgroup analysis of the patients with WHO-diagnosis was comparable to the full cohort results (Supplemental Tables S8.C \& S8.F).

\section{Survival}

Median OS was 8.9 months (95\% confidence interval (Cl) 4.1-13.6 months) for patients with Pd-I1+ tumors and 6.6 months (95\% Cl 5.6-7.6 months) for Pd-I1- tumors (HR 0.55, $95 \% \mathrm{Cl} 0.31-0.96, \mathrm{p}=0.038)$. No difference in survival in $\mathrm{Pd}-\mathrm{I} 1+$ high $(\geq 50 \%)$ or low (1-49\%) was observed (Figure 8.3). Positive staining of intra-tumor Cd8 was associated with improved OS compared to negative staining (7.9 months and 5.8 months, HR 0.62 (95\% Cl 0.40-0.94, $\mathrm{p}=0.026)$. Also, positive $\mathrm{Cd} 8$ staining in stromal cells was correlated with a longer OS (6.9 months vs. 4.0 months, HR 0.49 (95\% $\mathrm{Cl} 0.25-0.96), \mathrm{p}=0.037$ ) and a trend was seen for improved survival with a higher Cd8 density in stromal cells (Supplemental Figure S8.B). Results were comparable for the subgroup of patients achieving strict WHO criteria (Supplemental Figures S8.C \& S8.D). No differences were found in OS for IHC pRb or RB1, TP53, STK11 and KEAP1 mutation. Cox-regression included Pd-I1, Cd8 in stromal cells, age and gender and revealed HR $0.64(95 \% \mathrm{Cl} 0.36$ 1.16, $p=0.141)$. $C d 8$ in the tumor exhibited intersecting lines in the survival curve and was therefore excluded from cox-regression. Stratification for this factor revealed nonsignificant improved OS in Pd-I1 positive tumors in both subgroups (Supplemental Figure S8.E). 


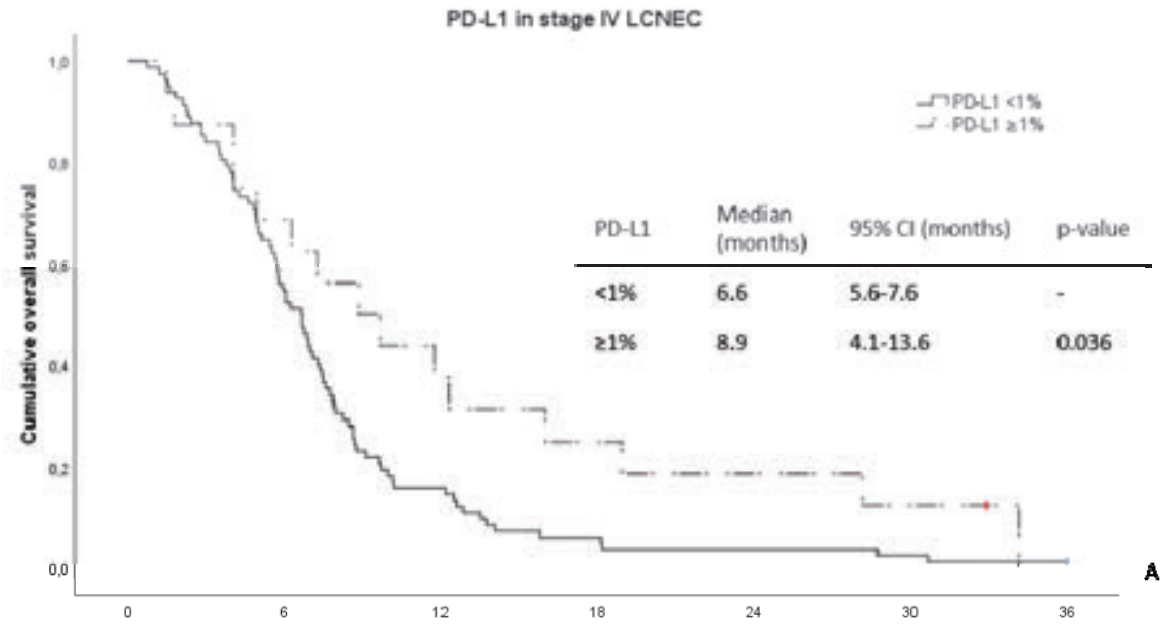

Number of patients at risk
\begin{tabular}{|l|l|l|l|l|l|l|l|}
\hline PD-L1+ & 16 & $\mathbf{1 1}$ & 6 & 4 & 3 & 2 & 0 \\
\hline PD-L1- & $\mathbf{8 2}$ & $\mathbf{4 5}$ & 13 & 5 & 3 & 2 & 1 \\
\hline
\end{tabular}

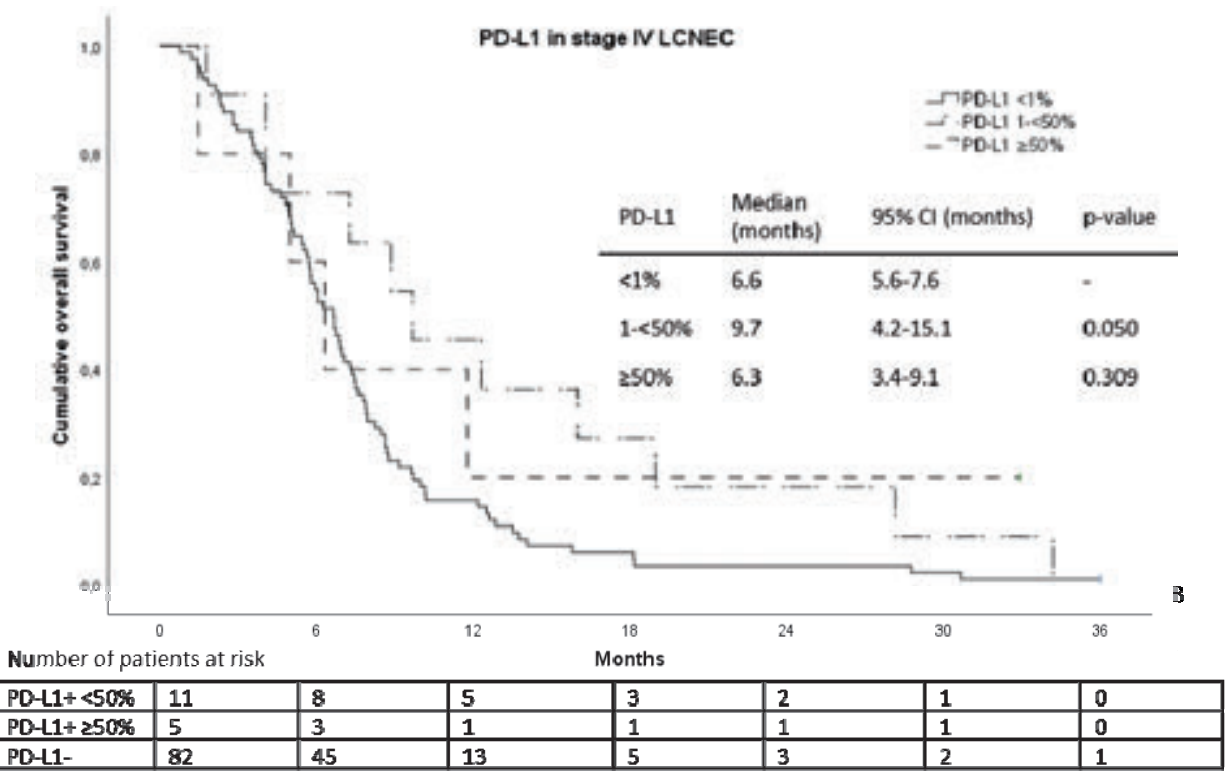

Figure 8.3 A) Overall survival for $\mathrm{Pd}-\mathrm{I} 1$ negative and positive tumors in stage IV LCNEC. B) Overall survival for Pd-I1 negative and positive tumors in stage IV LCNEC, subdivided in low $(<50 \%)$ and high ( $\geq 50 \%)$ Pd-I1 expression. 


\section{Discussion}

Pd-I1 expression in pre-treatment samples of LCNEC patients with metastatic disease has not yet been reported; there is scarce information on Pd-I1 expression in local disease. In this unique series of metastatic LCNEC we found Pd-I1 staining ( $\geq 1 \%$ ) in up to $16 \%$ of cases using the DAKO $28-8 \mathrm{IHC}$ antibody. Hence, based on Pd-I1 expression, combination therapy including Pd-I1 targeted therapy might be a successful extension of current therapy for LCNEC patients. However, this requires further clinical evaluation.

The Pd-I1 staining in LCNEC is comparable to reported values in SCLC, but distinctly lower than in NSCLC. ${ }^{12-14,16,18}$ Several studies have recently provided a similar prevalence of Pd-I1 staining in early stage LCNEC (9\% $(n=58), 10.4 \%(n=106), 16.7 \%$ $(n=72)$ and $22.4 \%(n=76)$ (Table 8.2)). ${ }^{19-22}$ However, three smaller studies revealed higher values of $20 \%(n=15), 27 \%(n=41)$ and $32 \%(n=28){ }^{23-25}$ Besides the size of cohorts, the use of different IHC Pd-I1 antibodies may explain differences in outcomes. We are the first to report the validated DAKO 28-8 antibody for staining in LCNEC. However, a blueprint study showed comparable results for usage of 22C3, SP263 and 28-8 in patients with NSCLC, whereas SP142 assay exhibited fewer stained tumor cells. No comparison was made for E1L3N and B7-H1 antibodies. ${ }^{33}$ Therefore, our results should at least be comparable with studies using 22C3 or SP263 antibodies. No explanation for variation is found in different thresholds defining Pd-I1 positivity (i.e. $\geq 5 \%$ instead of $\geq 1 \%$ ), since higher values were found with higher thresholds (Table $8.2)^{23-25}$

Recently, upregulation of immune related pathways has been reported in an LCNEC subgroup with TP53 and RB1 mutation. ${ }^{7}$ However, in this study Pd-I1 and Cd8 expression was similar in LCNEC with $R B 1$ mutated (SCLC-like) and $R B 1$ wildtype (NSCLC-like) tumors and although Pd-I1 expression is known to be distinctly higher in NSCLC compared to SCLC, this is not reflected when evaluating molecular LCNEC subgroups. Consistent with previous reports of lower $\mathrm{Pd}-\mathrm{I} 1$ expression and lower response rates to $\mathrm{Pd}-\mathrm{I} 1$ targeted therapy in patients with co-mutated KRAS and STK11 NSCLC, none of the seven STK11 mutated samples in our study harbored Pd-I1 expression and all had negative or limited ( $\leq 1 \%) \mathrm{Cd} 8$ staining. This might be due to the accumulation of neutrophils along with $T$ cell suppressive effects and $T$ cell exhaustion in STK11 mutated tumors. ${ }^{34-39}$ Since expression of Cd8 positive cells in the tumor is associated with Pd-I1 staining, this could clarify the reduced Pd-I1 expression in STK11 mutated tumors. Therefore, the effect of immunotherapeutic treatment might be 
reduced in STK11 mutated LCNEC and this should be taken into account in future clinical trials.

So far, conflicting results were presented for deviating survival in tumors expressing PdI1 in LCNEC. In this study, expression of any Pd-I1 was correlated with a superior OS (8.9 vs. 6.6 months). This is in accordance with previous reports by Inamura et al. and Tsuruoka et al. (Table 8.2). ${ }^{20,24}$ Contrary to our findings, Wang et al. reported a trend towards lower OS for total group of $\mathrm{Pd}-\mathrm{I} 1+$ pulmonary neuroendocrine carcinoma $(p=0.459)$. However, in multivariate analysis including clinical staging (I-III), Pd-I1 was not an independent prognostic factor. ${ }^{25}$ Also, a tendency to an inferior 5-year survival rate was revealed by Eichhorn et al.. Nevertheless, despite a higher prevalence of Pd-I1 staining in stage III and IV tumors, no multivariate analysis was reported. Therefore, the inferior survival might be related to a higher disease stage and not to Pd-I1 expression by itself. ${ }^{22}$ We included a more homogeneous population with only stage IV LCNEC, so our study is not affected by this confounding factor.

In this study, a minority of samples (16\%) had $>1 \%$ Cd8 positive cells in the tumor, while higher amounts were seen in the stromal cells (89\%). This may indicate that only a subgroup of LCNEC is an 'inflamed tumor', while the majority likely is 'immune excluded'. In those tumors, T-cell response is present, but T-cells do not seem to be able to penetrate the tumor. A positive correlation for intra-tumor $\mathrm{Cd} 8$ expressing cells and $\mathrm{Pd}-\mathrm{I} 1$ expression was found. A correlation between $\mathrm{Pd}-\mathrm{I} 1$ expression and $\mathrm{Cd} 8$ density in stromal cells has been reported previously. ${ }^{19,25}$ In this study, both positive Cd8 in T-cells in the tumor and in stromal cells were correlated with improved OS. In NSCLC patients, OS is also improved with increased Cd8 T-cell infiltration in both tumor cells and stromal cells (HR 0.77 (95\% Cl 0.66-0.93) and HR 0.77 (95\% Cl 0.69-0.86), respectively). ${ }^{40}$ For LCNEC patients, Wang et al. detected an improved OS with a higher Cd8 density in stromal cells (HR 2.77; 95\% Cl 1.29-5.93, p=0.009), however, association with OS was not found for Cd8 density in tumor cells. ${ }^{25}$ Kasajima et al. found a correlation between $\mathrm{Cd} 8$ density and higher immune cell infiltration, the latter resulting in a prolonged OS (37 vs. 80 months, $p=0.03$ ). ${ }^{19}$ Therefore, the improved OS we and others found in patients with Pd-I1 expression might be partly due to a more active immune system in those patients, reflected by $\mathrm{Cd} 8$. Although the tumor develops escape systems (i.e. Pd-I1) to resist the immune system, this inhibition seems to be only partial, preserving beneficial effects in at least part of the patients. In multivariate coxregression analysis in this study, including Cd8 positive cells in stroma, $\mathrm{Pd}-\mathrm{I} 1$ was not an independent prognostic factor. However, sample sizes for this analysis were small with only 10 patients in $\mathrm{Cd} 8$ negative group. 


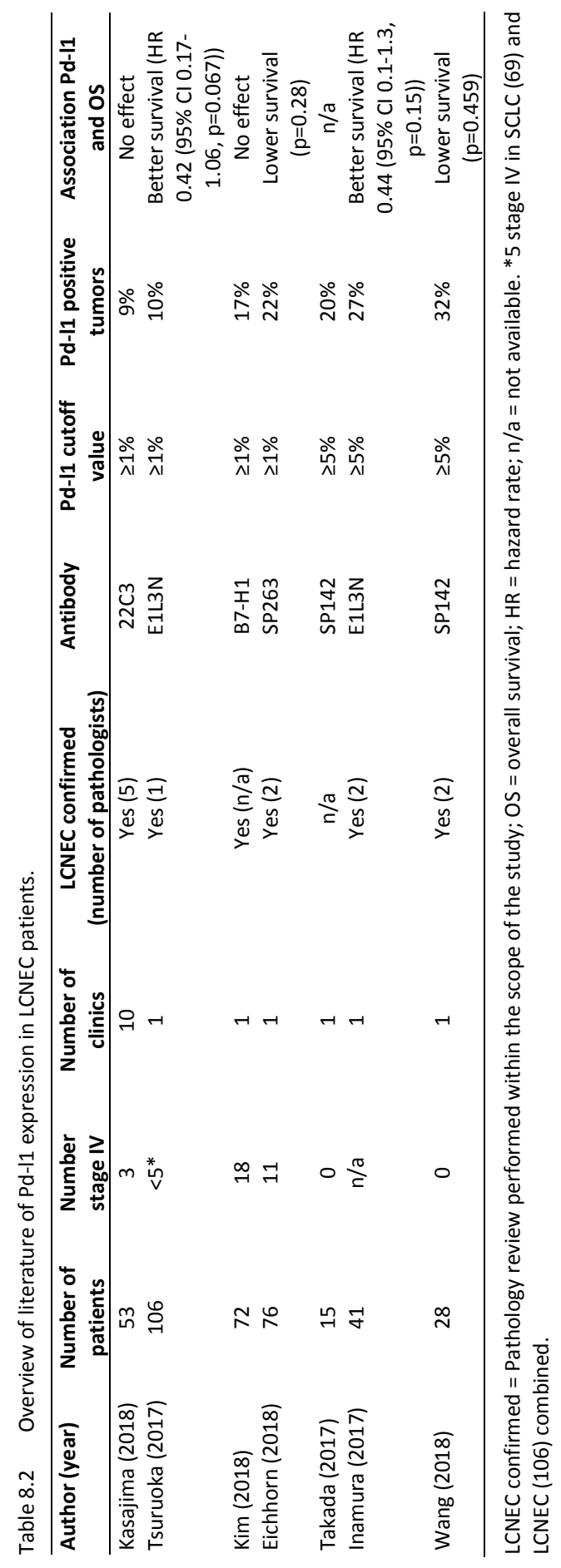


This study has some limitations. First, data was collected retrospectively and therefore we could not obtain all clinical characteristics of patients, i.e. smoking history or WHO performance score. Furthermore, most pathologic diagnoses were performed on biopsy samples, whereas it is known that it is difficult to diagnose LCNEC according to WHOcriteria on biopsy specimen. 5,32 However, the main problem for LCNEC diagnosis on biopsy specimen is lack of sensitivity, and not a lack of specificity. ${ }^{32}$ Subgroup analysis of the 85 patients with strict WHO-diagnosis was comparable to the results of full cohort (supplementary data). Another limitation is that we only established Pd-I1 in tumor cells, not in stromal cells. However, former studies revealed a positive correlation between $\mathrm{Cd} 8$ positive cells and $\mathrm{Pd}-\mathrm{I} 1$ expression in stromal cells, both as a measure of immune activity. ${ }^{19,25}$ Therefore, $\mathrm{Cd} 8$ can be considered as a reasonable alternative. Pd-I1 28-8 clone is known to show some background staining, but we have taken this into account and only scored membranous staining as positive.

Several small case series have reported responses (duration of response up to 6 months) to Pd-I1 monotherapy as second and later-line treatment in patients with LCNEC, irrespective of Pd-I1 expression. ${ }^{41-43}$ Furthermore, a response to nivolumab treatment was seen in few selected patients with SCLC having disease progression after at least one previous platinum-containing regimen. ${ }^{16}$ Based on these studies, Pd-I1 monotherapy might be suitable in LCNEC patients, irrespective of Pd-I1 expression. However, owing to relatively low levels of Pd-I1 expression and the high proportion of 'immune excluded' tumors with low Cd8 and Pd-I1 expression, combination with chemotherapy or another immunotherapy might be more appropriate. This is supported by recent results in first line treatment of SCLC where a combination of chemotherapy and atezolizumab showed a significant survival benefit. ${ }^{44}$ Another example of combination therapy is the improved response rate in SCLC patients treated with nivolumab and ipilimumab. ${ }^{26}$ In the future, more investigations including prospective trials are necessary to reveal the effect of $\mathrm{Pd}-\mathrm{I} 1 / \mathrm{Pd}-1$ inhibition in patients with LCNEC and the predictive value of $\mathrm{Pd}-\mathrm{I} 1, \mathrm{Cd} 8$ and/or tumor mutational burden.

In conclusion, this is the largest study so far reporting Pd-I1 expression in patients with well characterized stage IV LCNEC. Few patients had discernable Pd-I1 expression, with 5/98 high expressers, independent of molecular subtype. Patients with Pd-I1 expression had a better OS than Pd-I1 negative patients. Cd8 expression in T-cells in the tumor and stroma was correlated with Pd-I1 expression and improved OS. These results question the role of single agent $\mathrm{Pd}-(\mathrm{I}) 1$ inhibition in metastatic LCNEC and call for combination strategies. 


\section{References}

1. Derks JL, Hendriks LE, Buikhuisen WA, et al. Clinical features of large cell neuroendocrine carcinoma: a population-based overview. Eur Respir J 2016;47:615-624.

2. Takei $\mathrm{H}$, Asamura $\mathrm{H}$, Maeshima $\mathrm{A}$, et al. Large cell neuroendocrine carcinoma of the lung: a clinicopathologic study of eighty-seven cases. J Thorac Cardiovasc Surg 2002;124:285-292.

3. Asamura $\mathrm{H}$, Kameya $\mathrm{T}$, Matsuno $\mathrm{Y}$, et al. Neuroendocrine neoplasms of the lung: a prognostic spectrum. J Clin Oncol 2006;24:70-76.

4. Rossi G, Cavazza A, Marchioni A, et al. Role of chemotherapy and the receptor tyrosine kinases KIT, PDGFRalpha, PDGFRbeta, and Met in large-cell neuroendocrine carcinoma of the lung. J Clin Oncol 2005;23:8774-8785.

5. Travis WD, Brambilla E, Burke AP, et al. WHO Classification of Tumours of the Lung, Pleura, Thymus and Heart Lyon, France: International Agency for Research on Cancer; 2015.

6. Rekhtman N, Pietanza MC, Hellmann MD, et al. Next-Generation Sequencing of Pulmonary Large Cell Neuroendocrine Carcinoma Reveals Small Cell Carcinoma-like and Non-Small Cell Carcinoma-like Subsets. Clin Cancer Res 2016;22:3618-3629.

7. George J, Walter V, Peifer $\mathrm{M}$, et al. Integrative genomic profiling of large-cell neuroendocrine carcinomas reveals distinct subtypes of high-grade neuroendocrine lung tumors. Nat Commun 2018;9:1048.

8. Derks JL, Leblay N, Lantuejoul S, et al. New Insights into the Molecular Characteristics of Pulmonary Carcinoids and Large Cell Neuroendocrine Carcinomas, and the Impact on Their Clinical Management. J Thorac Oncol 2018;13:752-766.

9. Derks JL, Leblay N, Thunnissen E, et al. Molecular Subtypes of Pulmonary Large-cell Neuroendocrine Carcinoma Predict Chemotherapy Treatment Outcome. Clin Cancer Res 2018;24:33-42.

10. Novello S, Barlesi F, Califano R, et al. Metastatic non-small-cell lung cancer: ESMO Clinical Practice Guidelines for diagnosis, treatment and follow-up. Ann Oncol 2016;27:v1-v27.

11. Hanna N, Johnson D, Temin S, et al. Systemic Therapy for Stage IV Non-Small-Cell Lung Cancer: American Society of Clinical Oncology Clinical Practice Guideline Update. J Clin Oncol 2017;35: 3484-3515.

12. Mu CY, Huang JA, Chen Y, et al. High expression of PD-L1 in lung cancer may contribute to poor prognosis and tumor cells immune escape through suppressing tumor infiltrating dendritic cells maturation. Med Oncol 2011;28:682-688.

13. Chan AWH, Tong JHM, Kwan JSH, et al. Assessment of programmed cell death ligand-1 expression by 4 diagnostic assays and its clinicopathological correlation in a large cohort of surgical resected non-small cell lung carcinoma. Mod Pathol 2018 [Epub ahead of print].

14. Cooper WA, Tran T, Vilain RE, et al. PD-L1 expression is a favorable prognostic factor in early stage nonsmall cell carcinoma. Lung Cancer 2015;89:181-188.

15. Gandhi L, Rodriguez-Abreu D, Gadgeel S, et al. Pembrolizumab plus Chemotherapy in Metastatic NonSmall-Cell Lung Cancer. N Engl J Med 2018;378:2078-2092.

16. Antonia SJ, Lopez-Martin JA, Bendell J, et al. Nivolumab alone and nivolumab plus ipilimumab in recurrent small-cell lung cancer (CheckMate 032): a multicentre, open-label, phase 1/2 trial. Lancet Oncol 2016;17:883-895.

17. Ott PA, Elez E, Hiret S, et al. Pembrolizumab in Patients With Extensive-Stage Small-Cell Lung Cancer: Results From the Phase Ib KEYNOTE-028 Study. J Clin Oncol 2017;35:3823-3829.

18. Gadgeel SM, Pennell NA, Fidler MJ, et al. Phase II Study of Maintenance Pembrolizumab in Patients with Extensive-Stage Small Cell Lung Cancer (SCLC). J Thorac Oncol 2018;13:1393-1399.

19. Kasajima A, Ishikawa $\mathrm{Y}$, Iwata A, et al. Inflammation and PD-L1 expression in pulmonary neuroendocrine tumors. Endocr Relat Cancer 2018;25:339-350.

20. Tsuruoka K, Horinouchi H, Goto $\mathrm{Y}$, et al. PD-L1 expression in neuroendocrine tumors of the lung. Lung Cancer 2017;108:115-120. 
21. Kim HS, Lee JH, Nam SJ, et al. Association of PD-L1 Expression with Tumor-Infiltrating Immune Cells and Mutation Burden in High-Grade Neuroendocrine Carcinoma of the Lung. J Thorac Oncol 2018;13: 636-648.

22. Eichhorn F, Harms A, Warth A, et al. PD-L1 expression in large cell neuroendocrine carcinoma of the lung. Lung Cancer 2018;118:76-82.

23. Takada K, Toyokawa G, Okamoto T, et al. Metabolic characteristics of programmed cell death-ligand 1expressing lung cancer on (18) F-fluorodeoxyglucose positron emission tomography/computed tomography. Cancer Med 2017;6:2552-2561.

24. Inamura K, Yokouchi Y, Kobayashi M, et al. Relationship of tumor PD-L1 (CD274) expression with lower mortality in lung high-grade neuroendocrine tumor. Cancer Med 2017;6:2347-2356.

25. Wang H, Li Z, Dong B, et al. Prognostic significance of PD-L1 expression and CD8+ T cell infiltration in pulmonary neuroendocrine tumors. Diagn Pathol 2018;13:30.

26. Hellmann MD, Callahan MK, Awad MM, et al. Tumor Mutational Burden and Efficacy of Nivolumab Monotherapy and in Combination with Ipilimumab in Small-Cell Lung Cancer. Cancer Cell 2018;33:853861 e854.

27. Rizvi NA, Hellmann MD, Snyder A, et al. Cancer immunology. Mutational landscape determines sensitivity to PD-1 blockade in non-small cell lung cancer. Science 2015;348:124-128.

28. Snyder A, Makarov V, Merghoub T, et al. Genetic basis for clinical response to CTLA-4 blockade in melanoma. N Engl J Med 2014;371:2189-2199.

29. Derks JL, van Suylen RJ, Thunnissen E, et al. A Population-Based Analysis of Application of WHO Nomenclature in Pathology Reports of Pulmonary Neuroendocrine Tumors. J Thorac Oncol 2016;11:593-602.

30. Casparie M, Tiebosch AT, Burger G, et al. Pathology databanking and biobanking in The Netherlands, a central role for PALGA, the nationwide histopathology and cytopathology data network and archive. Cell Oncol 2007;29:19-24.

31. Derks JL, van Suylen RJ, Thunnissen E, et al. Chemotherapy for pulmonary large cell neuroendocrine carcinomas: does the regimen matter? Eur Respir J 2017;49.

32. Derks JL, Dingemans AC, van Suylen RJ, et al. Is the sum of positive neuroendocrine immunohistochemical stains useful for diagnosis of large cell neuroendocrine carcinoma (LCNEC) on biopsy specimen? Histopathology 2018; [Epub ahead of print].

33. Hirsch FR, McElhinny A, Stanforth D, et al. PD-L1 Immunohistochemistry Assays for Lung Cancer: Results from Phase 1 of the Blueprint PD-L1 IHC Assay Comparison Project. J Thorac Oncol 2017;12:208-222.

34. Saigi M, Alburquerque-Bejar JJ, Mc Leer-Florin A, et al. MET-Oncogenic and JAK2-Inactivating Alterations Are Independent Factors That Affect Regulation of PD-L1 Expression in Lung Cancer. Clin Cancer Res 2018;24(18):4579-4587.

35. Scheel AH, Ansen S, Schultheis AM, et al. PD-L1 expression in non-small cell lung cancer: Correlations with genetic alterations. Oncoimmunology 2016;5:e1131379.

36. Skoulidis F, Byers LA, Diao L, et al. Co-occurring genomic alterations define major subsets of KRASmutant lung adenocarcinoma with distinct biology, immune profiles, and therapeutic vulnerabilities. Cancer Discov 2015;5:860-877.

37. Skoulidis F, Goldberg ME, Greenawalt DM, et al. STK11/LKB1 Mutations and PD-1 Inhibitor Resistance in KRAS-Mutant Lung Adenocarcinoma. Cancer Discov 2018;8:822-835.

38. Rizvi H, Sanchez-Vega F, La K, et al. Molecular Determinants of Response to Anti-Programmed Cell Death (PD)-1 and Anti-Programmed Death-Ligand 1 (PD-L1) Blockade in Patients With Non-Small-Cell Lung Cancer Profiled With Targeted Next-Generation Sequencing. J Clin Oncol 2018;36:633-641.

39. Biton J, Mansuet-Lupo A, Pecuchet N, et al. TP53, STK11, and EGFR Mutations Predict Tumor Immune Profile and the Response to Anti-PD-1 in Lung Adenocarcinoma. Clin Cancer Res 2018;24(22): 5710-5723.

40. Soo RA, Chen Z, Yan Teng RS, et al. Prognostic significance of immune cells in non-small cell lung cancer: meta-analysis. Oncotarget 2018;9:24801-24820.

41. Levra MG, Mazieres J, C.A. V, et al. Efficacy of immune checkpoint inhibitors in large cell neuroendocrine lung cancer J Thorac Oncol 2017;12:S702-S703. 
42. Wang VE, Urisman A, Albacker L, et al. Checkpoint inhibitor is active against large cell neuroendocrine carcinoma with high tumor mutation burden. J Immunother Cancer 2017;5:75.

43. Daido W, Yamasaki M, Saito N, et al. [Effectiveness of Nivolumab in Large-Cell Neuroendocrine Carcinoma of the Lung - A Report of Two Cases]. Gan To Kagaku Ryoho 2017;44:59-62.

44. Horn L, Mansfield AS, Szczesna A, et al. First-Line Atezolizumab plus Chemotherapy in Extensive-Stage Small-Cell Lung Cancer. N Engl J Med 2018;379:2220-2229. 


\section{Supplemental material}

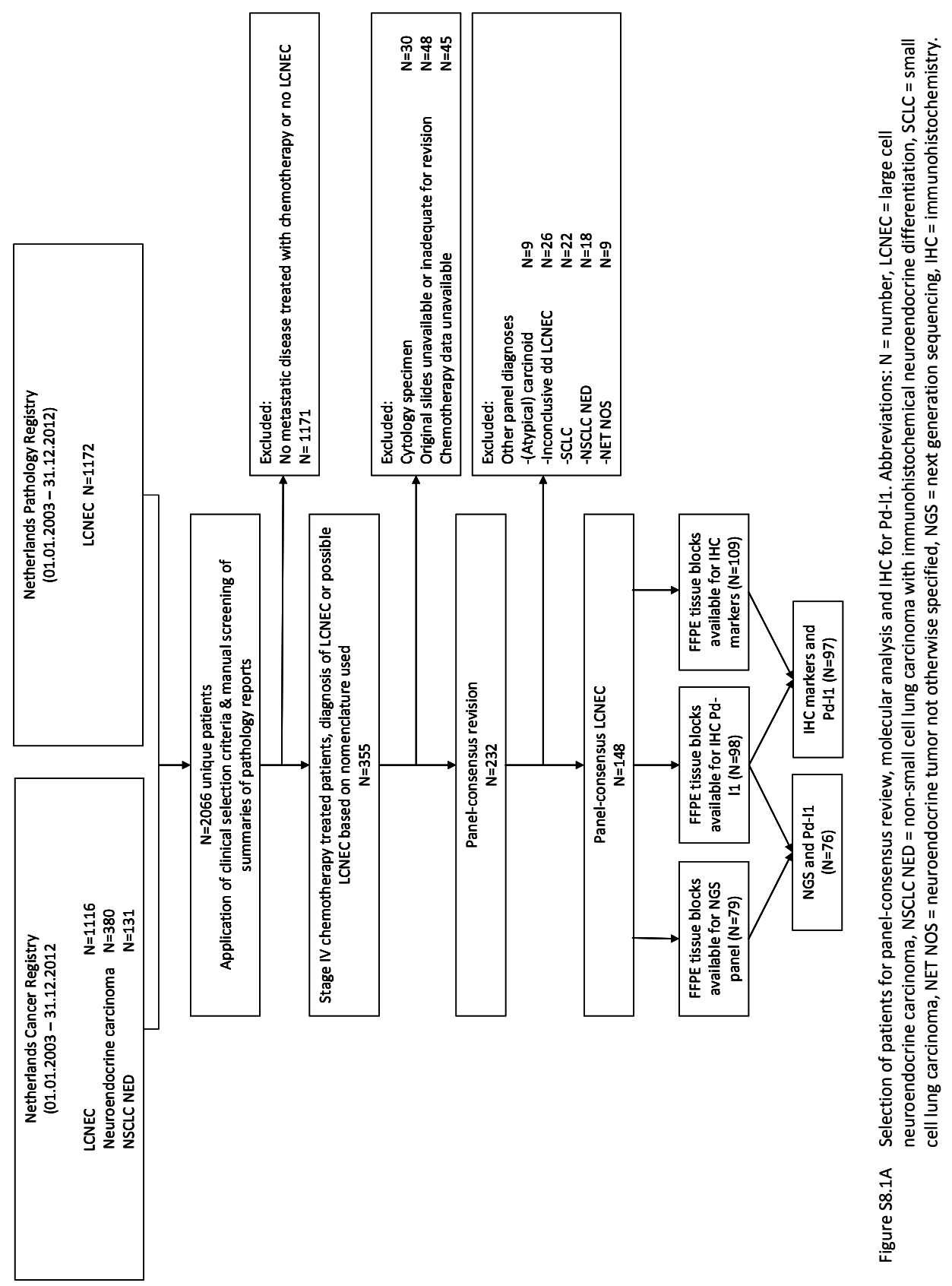




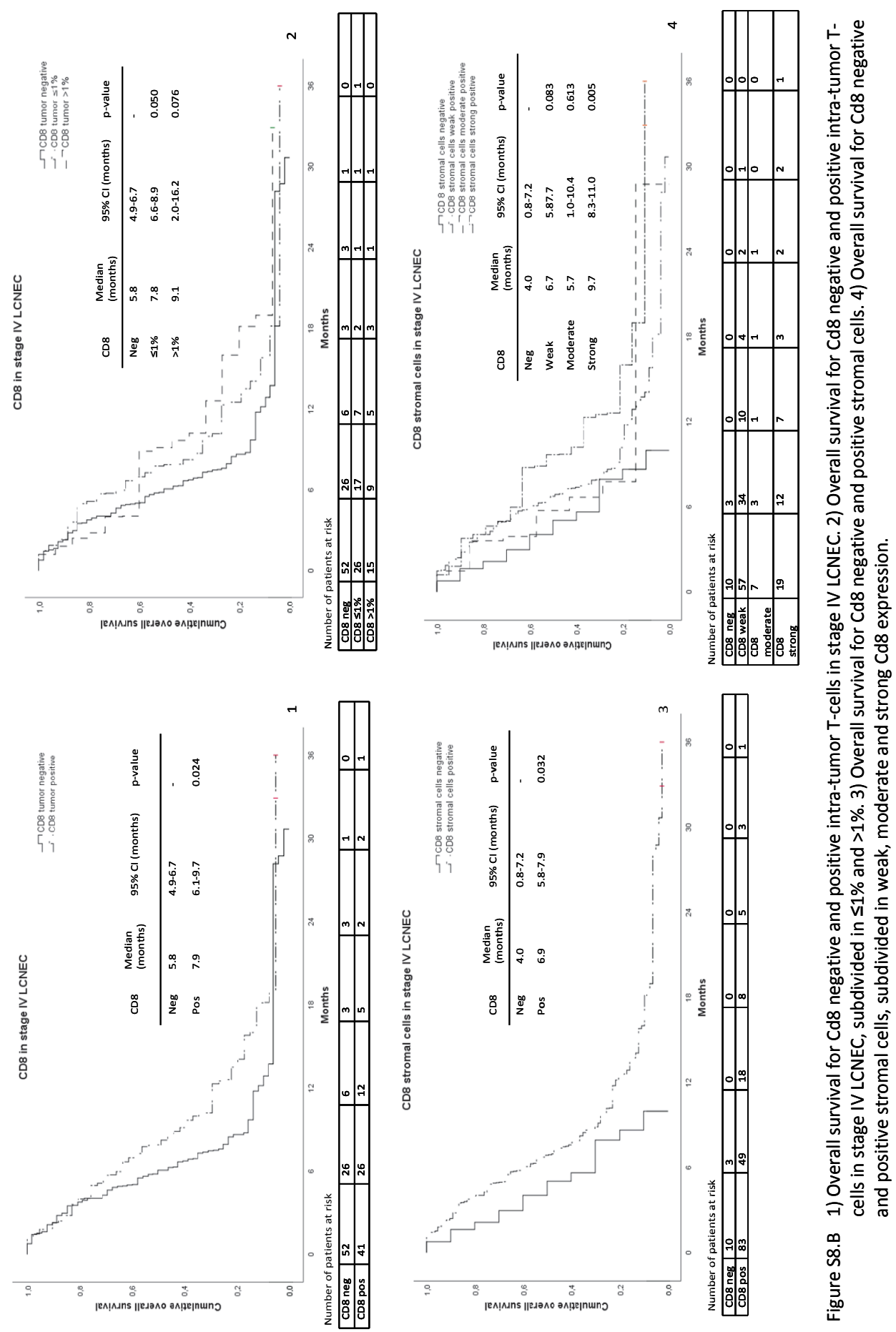



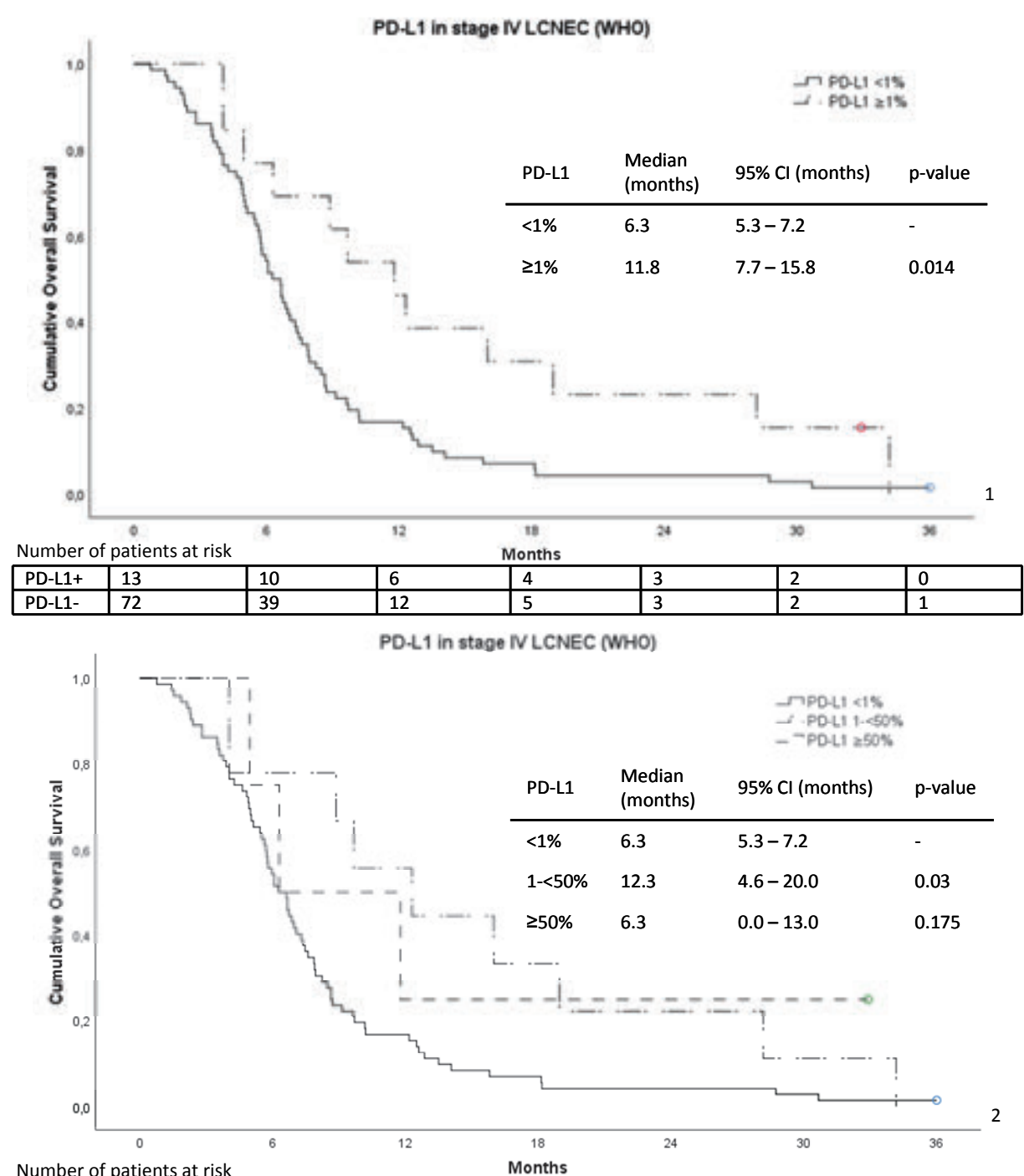

Number of patients at risk

\begin{tabular}{|l|l|l|l|l|l|l|l|}
\hline PD-L1+ $\geq 50 \%$ & 4 & 3 & 1 & 1 & 1 & 1 & 0 \\
\hline PD-L1+ $<50 \%$ & 9 & 7 & 5 & 3 & 2 & 1 & 0 \\
\hline PD-L1- & 72 & 39 & 12 & 5 & 3 & 2 & 1 \\
\hline
\end{tabular}

Figure S8.C Only patients with LCNEC diagnosis according to WHO criteria included. 1) Overall survival for Pd-I1 negative and positive tumors in stage IV LCNEC. 2) Overall survival for Pd-I1 negative and positive tumors in stage IV LCNEC, subdivided in low (<50\%) and high ( $\geq 50 \%)$ Pd-I1 expression. 


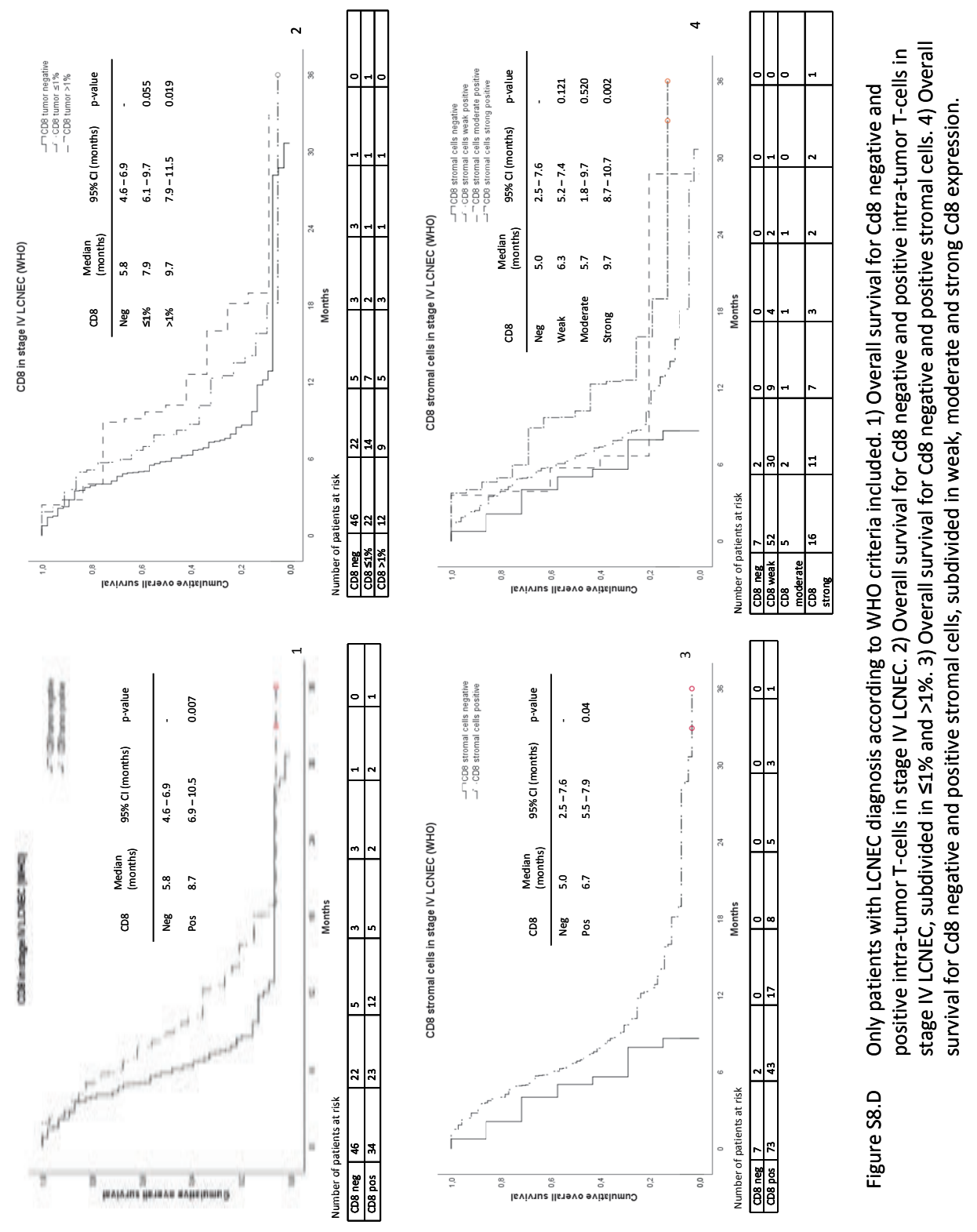


PD.L1 in CD8 negative LCNEC

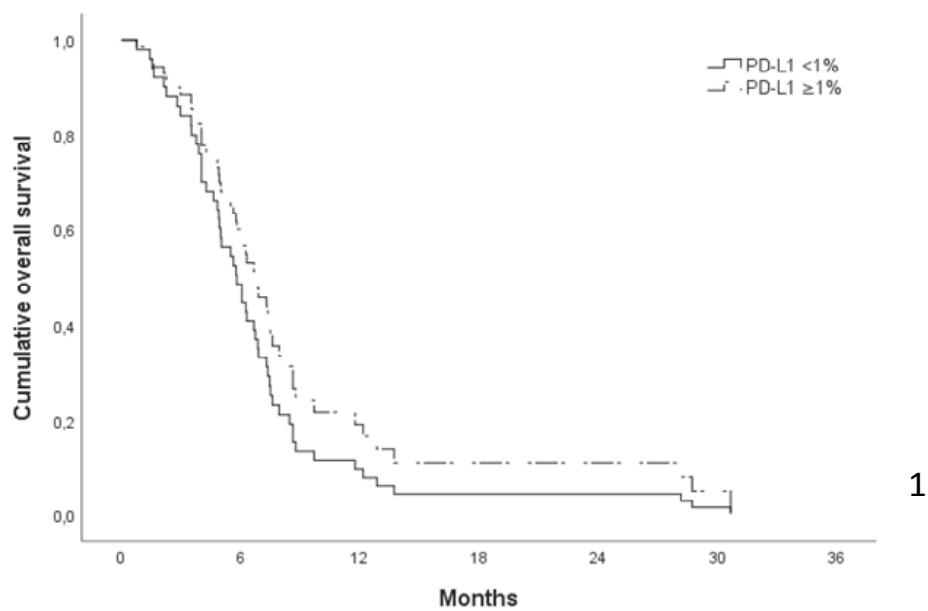

PD-L1 in CD8 positive LCNEC

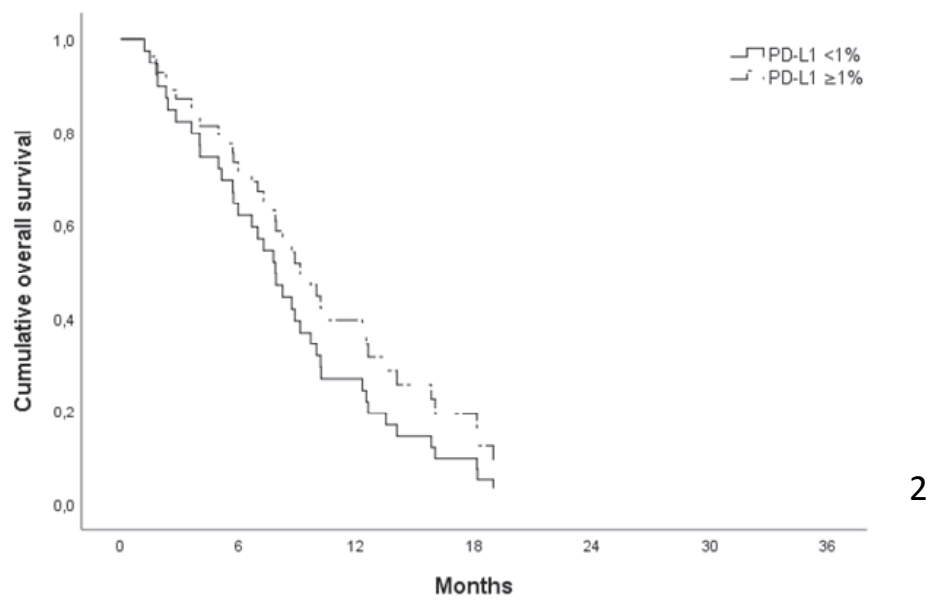

Figure S8.E Overall survival for Pd-I1 negative (1) and positive (2) tumors in stage IV LCNEC, stratified by Cd8 positivity in the tumor. HR $0.71(95 \% \mathrm{Cl} 0.39-1.29, \mathrm{p}=0.255)$. 
Table S8.A Expression of Pd-I1 in LCNEC (according to WHO criteria), patient characteristics and survival.

\begin{tabular}{lccc}
\hline & Pd-I1+ & Pd-l1- & p-value \\
\hline LCNEC (n=85) & $13(15 \%)$ & $72(85 \%)$ & - \\
$1-<50 \%$ & $9(11 \%)$ & - & - \\
$\geq 50 \%$ & $4(5 \%)$ & - & $0.902^{\#}$ \\
Age (median, range) & $60(37-74)$ & $64(34-82)$ & \\
Gender & & & \\
Male & $8(15 \%)$ & $44(85 \%)$ & 0.977 \\
Female & $5(15 \%)$ & $28(85 \%)$ & HR $0.46(0.25-0.86), p=0.015^{*}$ \\
OS in months (95\% Cl) & $11.8(7.7-15.8)$ & $6.3(5.3-7.2)$ &
\end{tabular}

${ }^{\#}$ Chi-square test (for age group $\leq 65$ and $>65$ ). ${ }^{*}$ Cox-regression including age and gender. Abbreviations: OS = overall survival; $95 \% \mathrm{Cl}=95 \%$ confidence interval; $\mathrm{HR}=$ hazard ratio.

Table S8.B Expression of Pd-I1 in LCNEC, correlated to molecular data \& Cd8 staining.

\begin{tabular}{|c|c|c|c|}
\hline & Pd-I1+ & Pd-I1- & p-value \\
\hline \multicolumn{4}{|l|}{ Mutation status ( $n=76$ ) } \\
\hline TP53 mutated $(\mathrm{N}=65)$ & $8(12 \%)$ & $57(88 \%)$ & $0.043^{\#}$ \\
\hline TP53 wildtype ( $\mathrm{N}=11)$ & $5(36 \%)$ & $7(64 \%)$ & \\
\hline$R B 1$ mutated $(\mathrm{N}=36)$ & $6(17 \%)$ & $30(83 \%)$ & $0.842^{\#}$ \\
\hline$R B 1$ wildtype $(\mathrm{N}=40)$ & $6(15 \%)$ & $34(85 \%)$ & \\
\hline STK11 mutated $(\mathrm{N}=7)$ & $0(0 \%)$ & $7(100 \%)$ & $0.229^{\#}$ \\
\hline STK11 wildtype ( $\mathrm{N}=69)$ & $12(17 \%)$ & $57(82 \%)$ & \\
\hline KEAP1 mutated $(\mathrm{N}=13)$ & $2(15 \%)$ & $11(85 \%)$ & $0.965^{\#}$ \\
\hline KEAP1 wildtype $(\mathrm{N}=63)$ & $10(16 \%)$ & $53(84 \%)$ & \\
\hline \multicolumn{4}{|l|}{ IHC pRb (n=97) } \\
\hline pRb (normal expression) ( $n=29)$ & $3(10 \%)$ & $26(90 \%)$ & $0.287^{\#}$ \\
\hline pRb (no expression) (n=68) & $13(19 \%)$ & $55(81 \%)$ & \\
\hline \multicolumn{4}{|l|}{ IHC Cd8 (n=93) } \\
\hline Cd8+ in tumor $(n=41)$ & $11(27 \%)$ & $30(73 \%)$ & $0.013^{\#}$ \\
\hline Cd8- in tumor $(n=52)$ & $4(8 \%)$ & $48(92 \%)$ & \\
\hline Cd8+ in stromal cells $(n=83)$ & $15(18 \%)$ & $68(82 \%)$ & $0.142^{\#}$ \\
\hline Cd8- in stromal cells $(n=10)$ & $0(0 \%)$ & $10(100 \%)$ & \\
\hline
\end{tabular}

$\mathrm{IHC}=$ immunohistochemistry; ${ }^{*}$ Chi-square test/Fisher's Exact Test. 
Table S8.C Expression of Pd-I1 in LCNEC (according to WHO criteria), correlated to molecular data \& Cd8 staining.

\begin{tabular}{|c|c|c|c|}
\hline & Pd-I1+ & Pd-I1- & p-value \\
\hline \multicolumn{4}{|l|}{ Mutation status ( $n=69$ ) } \\
\hline TP53 mutated $(\mathrm{N}=58)$ & $6(10 \%)$ & 52 (90\%) & $0.025^{\#}$ \\
\hline TP53 wildtype ( $\mathrm{N}=11)$ & $4(36 \%)$ & $7(64 \%)$ & \\
\hline RB1 mutated $(\mathrm{N}=32)$ & $6(19 \%)$ & $26(81 \%)$ & $0.350^{\#}$ \\
\hline RB1 wildtype (N=37) & $4(11 \%)$ & $33(89 \%)$ & \\
\hline STK11 mutated ( $\mathrm{N}=7)$ & $0(0 \%)$ & $7(100 \%)$ & $0.251^{\#}$ \\
\hline STK11 wildtype ( $\mathrm{N}=62)$ & $10(16 \%)$ & $52(84 \%)$ & \\
\hline KEAP1 mutated $(\mathrm{N}=13)$ & $2(15 \%)$ & $11(85 \%)$ & $0.919^{\#}$ \\
\hline KEAP1 wildtype $(\mathrm{N}=56)$ & $8(14 \%)$ & $48(86 \%)$ & \\
\hline \multicolumn{4}{|l|}{ IHC pRb $(n=84)$} \\
\hline $\mathrm{pRb}$ (normal expression) ( $\mathrm{n}=24)$ & $2(8 \%)$ & $22(92 \%)$ & $0.252^{\#}$ \\
\hline pRb (no expression) $(n=60)$ & $11(18 \%)$ & 49 (82\%) & \\
\hline \multicolumn{4}{|l|}{ IHC Cd8 (n=80) } \\
\hline $\mathrm{Cd} 8+$ in tumor $(n=34)$ & $8(24 \%)$ & $26(77 \%)$ & $0.066^{\#}$ \\
\hline Cd8- in tumor $(n=46)$ & $4(9 \%)$ & 42 (91\%) & \\
\hline Cd8+ in stromal cells $(n=73)$ & $12(16 \%)$ & $61(84 \%)$ & $0.245^{\#}$ \\
\hline Cd8- in stromal cells $(n=7)$ & $0(0 \%)$ & $7(100 \%)$ & \\
\hline
\end{tabular}

$\mathrm{IHC}=$ immunohistochemistry; ${ }^{\#}$ Chi-square test/Fisher's Exact Test.

Table S8.D Cd8 staining in tumor and stromal cells.

\begin{tabular}{lc}
\hline Cd8 within tumor $(\mathbf{n}=\mathbf{9 3})$ & \\
Negative & $52(56 \%)$ \\
$\leq 1 \%$ & $26(28 \%)$ \\
$>1 \%$ & $15(16 \%)$ \\
Cd8 in stromal cells $(\mathbf{n = 9 3 )}$ & \\
Negative & $10(11 \%)$ \\
Weak positive & $57(61 \%)$ \\
Moderate positive & $7(8 \%)$ \\
Strong positive & $19(20 \%)$ \\
\hline
\end{tabular}

Table S8.E Cd8 within tumor (cells $/ \mathrm{mm}^{2}$ ), only if estimated at $>1 \%$

\begin{tabular}{lc}
\hline Patient & Cells $/ \mathbf{m m}^{2}$ \\
\hline 1 & 100 \\
2 & 86 \\
3 & 184 \\
4 & 57 \\
5 & 58 \\
6 & 38 \\
7 & 15 \\
8 & 196 \\
9 & 321 \\
10 & 300 \\
11 & 103 \\
12 & 154 \\
13 & 54 \\
14 & 83 \\
15 & 376 \\
\hline
\end{tabular}


Table S8.F Cd8 staining in tumor and stromal cells (LCNEC according to WHO criteria)

\begin{tabular}{lc}
\hline Cd8 within tumor $(\mathbf{n}=\mathbf{8 0})$ & \\
Negative & $46(58 \%)$ \\
$\leq 1 \%$ & $22(28 \%)$ \\
$>1 \%$ & $12(15 \%)$ \\
Cd8 in stromal cells $(\mathbf{n}=\mathbf{8 0})$ & \\
Negative & $7(9 \%)$ \\
Weak positive & $52(65 \%)$ \\
Moderate positive & $5(6 \%)$ \\
Strong positive & $16(20 \%)$ \\
\hline
\end{tabular}





\section{Chapter 9}

\section{Unique metastatic patterns in neuroendocrine neoplasms of different primary origin}

B.C.M. Hermans, J. de Vos-Geelen, J.L. Derks, L. Latten, I.H. Liem, J.M. van der Zwan, E.J.M. Speel, M.W. Dercksen, A-M.C. Dingemans Neuroendocrinology 2020 Nov 23. Online ahead of print. 


\section{Abstract}

\section{Background}

Neuroendocrine neoplasms (NEN) can originate in different organs, e.g. the gastroenteral tract (GE), pancreas (Pan) or lung (L). Our aim was to examine metastatic patterns for patients with NEN of various primary origins with a special focus on brain metastases to indicate utility for screening.

\section{Methods}

All NEN patients except for small cell lung cancer registered in the Netherlands Cancer Registry from 2008-2018 were selected. Metastatic patterns at initial diagnosis for NEN with different primary origin were compared. In a subcohort of patients from two referral hospitals (2014-2019), additional information on for example development of metastases after initial presentation was available.

\section{Results}

In the nationwide cohort 4,768/11,120 (43\%) patients had metastatic disease at diagnosis (GE 1,504/4,710 (32\%), Pan 489/1,150 (43\%), L 1,230/2,978 (41\%)). For GEand Pan-NEN, the most prevalent metastatic site was the liver ( $25 \%$ and $39 \%$ ), followed by distant lymph nodes ( $8 \%$ and $8 \%$ ), whereas only few patients with brain metastases were identified ( $0 \%$ in both). In contrast, for L-NEN, prevalence of metastases in liver $(19 \%)$, brain (9\%), lung (7\%) and bone (14\%) was more equal. In the reference network cohort, slightly more NEN patients had metastatic disease $(260 / 539,48 \%)$ and similar metastatic patterns were observed.

\section{Conclusion}

Almost half of NEN patients were diagnosed with synchronous metastatic disease. L-NEN have a unique metastatic pattern compared to GE- and Pan-NEN. Remarkably, an important part of L-NEN metastases were in the brain, whereas brain metastases were almost absent in GE- and Pan-NEN, indicating utility of screening in L-NEN. 


\section{Introduction}

Neuroendocrine neoplasms (NEN) constitute a heterogeneous group of neoplasms with a histopathological neuroendocrine appearance as their typical hallmark. NEN can originate in different anatomical locations, e.g. gastroenteral tract, pancreas, and lungs. ${ }^{1-3}$ NEN are subdivided in low/intermediate grade neuroendocrine tumors (NET) and high grade neuroendocrine carcinomas (NEC). ${ }^{1-3}$ In general, NEC have an aggressive behavior whereas the course of NET might be more indolent with higher survival rates. $^{1-3}$

Metastases are found in up to $50 \%$ of all NEN patients with the liver as the most frequent metastatic site (up to $85 \%$ of all metastases). ${ }^{4-11}$ So far, metastatic patterns in NET from different primary organs have only been extensively described by Riihimaki et al. in 7,334 patients. ${ }^{11}$ They found the liver as the most prominent site in gastroenteral and pancreatic NET ( $20 \%$ and $54 \%$ of all patients at diagnosis), with other metastases (e.g. lung and bone) at a maximum of $10 \%$. In contrast, incidence of liver metastases at diagnosis in lung NET patients was only $10 \%$, whereas lung (e.g. contralateral lesion), bone and nervous system metastases also constituted an important part in this subgroup. ${ }^{11}$ An important limitation of this study is the lack of data on tumor grade. Furthermore, NEC have been excluded from this analysis. ${ }^{11}$

Only limited data considering the clinical relevance of brain metastases in NEN is available. $^{12}$ In the majority of patients, dissemination of the tumor is investigated by a ${ }^{18}$ F-Fluorodeoxyglucose positron emission tomography (FDG-PET) or somatostatin receptor targeting scan (e.g. ${ }^{68}$ Ga-DOTATATE-PET, ${ }^{68}$ Ga-DOTATOC-PET or ${ }^{111} \mathrm{In}$ penetreotide scintigraphy). FDG-PET is insensitive for the detection of brain metastases, because of high physiological brain glucose metabolism. Somatostatin receptor targeting scans might be able to show brain lesions, however, differentiation between meningioma and metastases can be difficult. ${ }^{13}$ In the most common NEC, small cell lung carcinoma (SCLC), brain metastases are frequently present and therefore, patients eligible for curative therapy are screened for asymptomatic brain metastases with magnetic resonance imaging (MRI) or computed tomography (CT) scans. ${ }^{14,15}$ For small cell NEC of other primary origins and for large cell NEC or low/intermediate grade NET, guidelines do not advice on brain metastases screening. However, brain metastases have been described for NEN apart from SCLC and presence of (a)symptomatic brain metastases might influence prognosis and therapeutic choices. $^{10-12,15}$ 
Most medical oncologists and endocrinologists treat patients with NEN from various primary origins and different grades. This could result in suboptimal treatment plans for less prevalent NEN (e.g. low grade pulmonary NET), since the use of clinical experience with more prevalent NEN (e.g. low grade gastroenteral NET) might be unjustified because of different clinical, histopathological or molecular characteristics. Therefore, insight in similarities and differences between various NEN will contribute to optimal treatment to every unique patient. In this study, we describe metastatic patterns in patients with NEN of various primary origins and tumor grade and investigate the effect of primary origin and metastatic sites on overall survival. Furthermore, we particularly focus on the incidence of brain metastases to indicate utility of cerebral screening in different types of NEN.

\section{Methods}

\section{Cohort national cancer registry}

\section{Patient selection}

The first cohort of this study was selected from the Netherlands Cancer Registry (NCR). Specialized data managers collected patient data for this database with a nationwide coverage $>95 \% .{ }^{16,17}$ A yearly linkage to the Centralized Civil Registry ensures up-to-date data on overall survival. In this cohort, all patients diagnosed with NEN (except SCLC) in the Netherlands between January 1, 2008 and December 31, 2018 were selected including similar morphology codes (International Classification of Diseases for Oncology) as described by Korse et al.. ${ }^{17}$ Patients with another malignancy before or concurrent with the NEN diagnosis were excluded, since in these cases the registered metastases could also originate from the other primary malignancy (Supplemental Figure S9.A). Anonymous data on patient characteristics (gender, age), primary tumor characteristics (primary origin, grade), metastatic status at diagnosis (including metastatic sites) and survival data were available. Patients were excluded if topography of metastatic sites was not available.

\section{Subgroup formation}

For analysis of the primary tumor, 5 subgroups were created: 1) Gastroenteral (GE), including esophagus, stomach, duodenum, jejunum, ileum, appendix, colon and rectum, 2) Pancreas (Pan), 3) Lung (L), 4) Other (O), including amongst others Merkel cell carcinoma (MCC), mesenterial tumors, thymus NEN and NEN from the urogenital 
system, and 5) Unknown (U). Three groups were created for tumor grade: 1) grade 1 (G1, including pulmonary typical carcinoid), 2) grade 2 (G2, including pulmonary atypical carcinoid) and 3 ) grade 3 (G3, including pulmonary large cell neuroendocrine carcinoma (LCNEC) and both grade 3 NET and NEC for GEP-NEN). Metastatic sites were categorized in: 1) Liver metastases 2) Brain metastases, 3) Lung metastases, 4) Bone metastases, 5) Distant lymph node metastases, 6) Peritoneal metastases, and 7) Other metastases (including metastases of pleura, skin, soft tissue and adrenal glands).

\section{Presentation of data and statistical analysis}

All statistical analyses were performed with IBM SPSS Statistics, version 25 for Windows, Armonk, NY: IBM Corp.. Metastatic patterns at diagnosis are presented for the different primary tumors, with a subdivision for tumor grade. Median overall survival (OS) was evaluated by Kaplan-Meier analysis for the total group of metastatic patients and for metastatic G1, G2 and G3 patients separately. Differences in OS, indicating prognostic factors, were tested for significance with Log-Rank test and presented as hazard ratios (HR) with $95 \%$ confidence interval $(95 \% \mathrm{Cl}$ ). Investigated variables were: gender (male, female), age $(\leq 65,>65)$, primary tumor (gastroenteral, pancreas, lung, other, unknown), tumor grade (grade 1, 2, 3), number of organs with metastases (1, 2-3, 24), and liver, brain, lung, bone and distant lymph node metastases (yes, no). Variables with a $p$-value $<0.10$ in univariable analysis were selected for multivariable Cox regression analysis. Variables with a $p$-value $>0.10$ were only included in multivariable analysis if they were regarded highly clinical relevant (i.e. metastatic sites). Interaction was tested between different metastatic sites included in the multivariable model. Interaction terms were included in the multivariable model if Omnibus Tests of Model Coefficients showed a difference between -2 Log Likelihoods of the models $(p<0.05)$. To prevent overfitting, we adopted an event per variable ratio of $\geq 10$. Results of the full multivariable model are presented and a $p$-value $<0.05$ is regarded statistically significant.

\section{Cohort reference network}

For the second cohort of this study, all patients diagnosed and/or treated with a NEN (except for SCLC) between January 1, 2014 and December 31, 2019 in two NEN referral centers in the Netherlands (Maastricht University Medical Centre and Maxima Medical Centre) were selected. Most of the patients in this cohort were also included in the nationwide cohort, but this subcohort could provide us with more in depth information, e.g. imaging used for diagnostic workup and development of metastases in patients with local disease at diagnosis. Patients with unclear metastatic status at diagnosis or 
who objected against use of their data for medical research were excluded (Supplemental Figure S9.A). Data on patient and tumor characteristics, staging procedures, treatment, survival, and follow-up were retrieved from medical records. Subgroup formation was performed as described for the nationwide cohort. Metastatic disease was in general evaluated with computed tomography (CT)-thorax/upper abdomen for all lung NEN and CT-thorax/abdomen for GEP-NEN, with a simultaneous or additional FDG-PET and/or somatostatin receptor targeting scan, if necessary. ${ }^{18-21}$ For NEN of other primary origins, work-up depends on the primary tumor. Metastatic patterns at initial presentation are presented for different primary tumor sites, with a subdivision for tumor grade. Furthermore, for patients without metastases at diagnosis, metastatic patterns during follow-up are presented for the different primary origins. The medical ethical review committee of Maastricht University Medical Centre+ assessed this study as not being subject to the Medical Research Involving Human Subjects Act (WMO) and the study was approved by the board of directors of Maastricht University Medical Centre+ (METC 2018-0911, January 24, 2019).

\section{Results}

\section{Cohort national cancer registry}

\section{Study population and metastases}

Between 2008 and 2018, 14,443 NEN patients were registered by the Netherlands Cancer Registry. A total of 3,318 patients were excluded because of a former or concurrent second malignancy and 5 patients were excluded since no information on topography of metastases was available (Supplemental Figure S9.A). Out of 11,120 included patients, 4,768 (43\%) had metastatic disease at initial presentation. Number of patients in each subgroup and patient characteristics can be found in Figure 9.1 and Table 9.1. In GE- and Pan-NEN most patients presented with grade 1 disease $(69 \%$ and $46 \%)$, but L-NEN, O-NEN and U-NEN presented most often with grade 3 disease (59\%, $82 \%$ and 65\%) (Figure 9.1, Supplemental Table S9.A). 
Table 9.1 Patient characteristics of all patients with neuroendocrine neoplasms and for subgroups of different primary origins.

\begin{tabular}{|c|c|c|c|c|c|c|}
\hline & All (\%) & GE (\%) & Pancreas (\%) & Lung (\%) & Other (\%) & Unknown (\%) \\
\hline \multicolumn{7}{|c|}{ Cohort national cancer registry } \\
\hline Total number & 11,120 & 4,710 & 1,150 & 2,978 & 1,075 & 1,207 \\
\hline \multicolumn{7}{|l|}{ Gender } \\
\hline Male & $5,588(50)$ & $2,356(50)$ & $594(52)$ & $1,442(48)$ & $580(54)$ & $616(51)$ \\
\hline Female & $5,532(50)$ & $2,354(50)$ & $556(48)$ & $1,536(52)$ & $495(46)$ & $591(49)$ \\
\hline \multicolumn{7}{|l|}{ Age } \\
\hline$\leq 65$ & $6,354(57)$ & $2,984(63)$ & $732(64)$ & $1,640(55)$ & $515(48)$ & $483(40)$ \\
\hline$>65$ & $4,766(43)$ & $1,726(37)$ & $418(36)$ & $1,338(45)$ & $560(52)$ & $724(60)$ \\
\hline \multicolumn{7}{|c|}{ Cohort reference network } \\
\hline Total number & 539 & 219 & 96 & 103 & 80 & 41 \\
\hline \multicolumn{7}{|l|}{ Gender } \\
\hline Male & $276(51)$ & $108(49)$ & $49(51)$ & $48(47)$ & $45(56)$ & $26(63)$ \\
\hline Female & $263(49)$ & $111(51)$ & 47 (49) & $55(53)$ & $35(44)$ & $15(37)$ \\
\hline \multicolumn{7}{|l|}{ Age } \\
\hline$\leq 65$ & 277 (51) & $124(57)$ & $52(54)$ & $55(53)$ & $30(38)$ & $16(39)$ \\
\hline$>65$ & $262(49)$ & $95(43)$ & $44(46)$ & $48(47)$ & $50(63)$ & $25(61)$ \\
\hline \multicolumn{7}{|l|}{ WHO PS } \\
\hline $0-1$ & $408(76)$ & $173(79)$ & 76 (79) & $77(75)$ & $54(68)$ & $28(68)$ \\
\hline$\geq 2$ & $22(4)$ & $4(2)$ & $1(1)$ & $5(5)$ & $4(5)$ & $8(20)$ \\
\hline Unknown & $109(20)$ & $42(19)$ & $19(20)$ & $21(20)$ & $22(28)$ & $5(12)$ \\
\hline
\end{tabular}

Abbreviations: GE = gastroenteral; WHO PS = World Health Organization Performance Score.

\section{Metastatic patterns at diagnosis}

The liver was the most frequent site of metastatic disease in GE-NEN $(1,158 / 4,710$, 25\%), whereas lung and bone metastases were rare (Figure 9.2, Supplemental Table S9.A). The same pattern was seen in Pan-NEN with liver metastases in 447/1,150 (39\%) of new cases. Brain metastases were observed in 7/4,710 (0.1\%) GE-NEN and 4/1,150 $(0.3 \%)$ Pan-NEN. The liver was also the most prevalent metastatic site in L-NEN $(568 / 2,978,19 \%)$, but brain, lung and bone metastases were also present in a substantial number of patients ( $9 \%, 7 \%$ and $14 \%$, respectively). In O-NEN, liver, bone and lymph node metastases were most frequent (15\%, $13 \%$ and $13 \%$, respectively). The majority of U-NEN presented with liver metastases (66\%), followed by lymph node metastases (39\%) (Figure 9.2, Supplemental Table S9.A). 

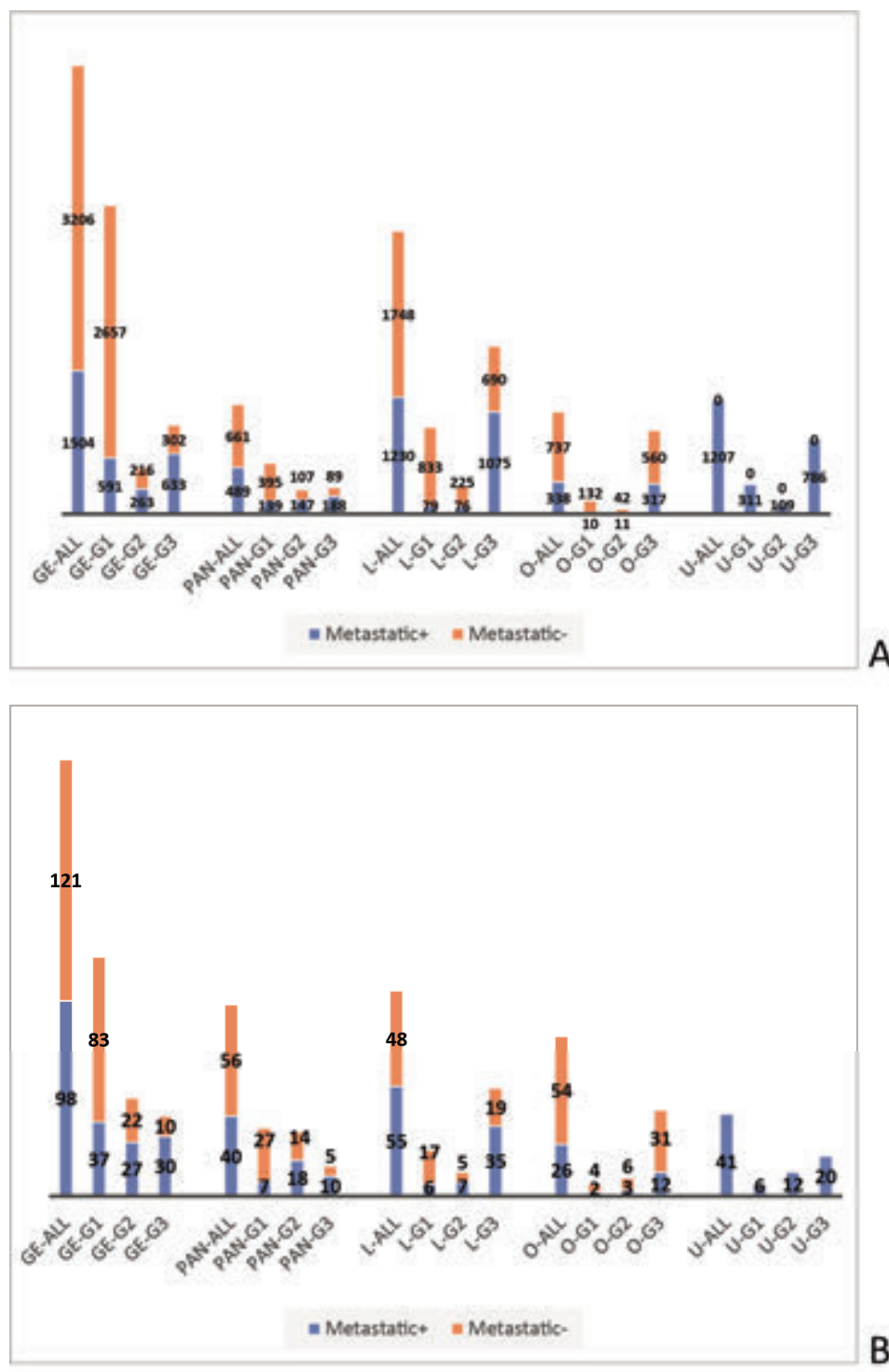

Figure 9.1 Number of patients with metastatic and non-metastatic neuroendocrine neoplasms of different primary origins and tumor grades in the cohort national cancer registry $(A)$ and the cohort reference network (B). Abbreviations: $\mathrm{GE}-\mathrm{ALL}=$ total cohort of gastroenteral neuroendocrine neoplasms; $\mathrm{GE}-\mathrm{G1}=$ gastroenteral grade $1 ; \mathrm{GE}-\mathrm{G} 2$ = gastroenteral grade $2 ; \mathrm{GE}-\mathrm{G} 3=$ gastroenteral grade $3, \mathrm{PAN}-\mathrm{ALL}=$ total cohort of pancreatic neuroendocrine neoplasms; $\mathrm{L}-\mathrm{ALL}=$ total cohort of lung neuroendocrine neoplasms; $\mathrm{O}-\mathrm{ALL}=$ total cohort of other neuroendocrine neoplasms; $\mathrm{U}-\mathrm{ALL}=$ total cohort of neuroendocrine neoplasms with unknown primary origin. 


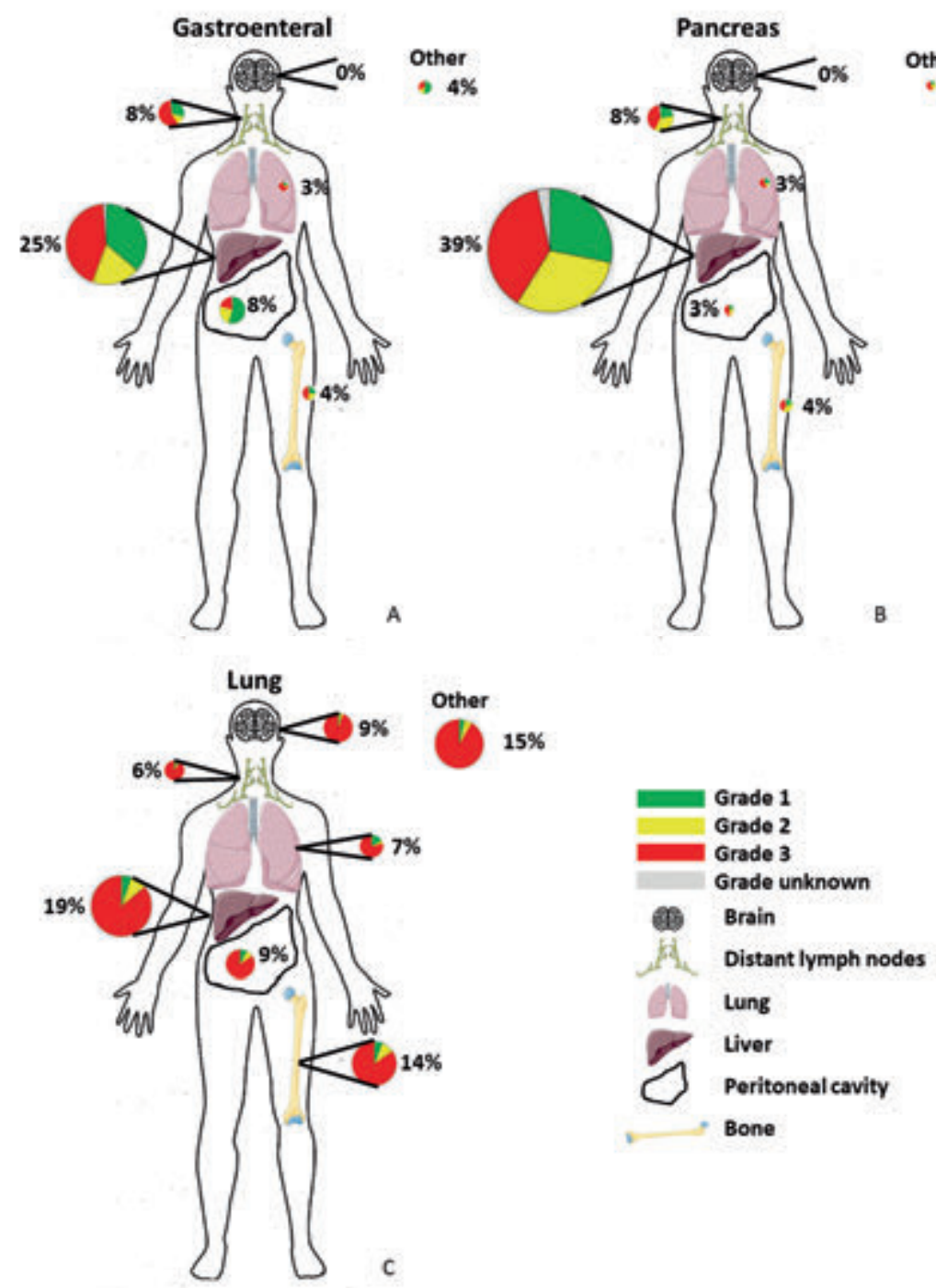

Figure 9.2 Metastatic patterns in neuroendocrine neoplasms of different primary origins in the cohort national cancer registry. Percentage of patients with liver, brain, lung, bone, distant lymph nodes, peritoneal cavity, and other metastases at diagnosis is presented, in relation to the total group of patients (both metastatic and non-metastatic). Percentages can add up above the total percentage of metastatic patients, since the majority of patients have metastases in multiple organs. A) Gastroenteral neuroendocrine neoplasms ( $N=4,710)$. B) Pancreatic neuroendocrine neoplasms $(\mathrm{N}=1,150)$. C) Pulmonary neuroendocrine neoplasms $(\mathrm{N}=2,978)$. 


\section{Overall survival}

In non-metastatic patients median OS was not reached for all grades together, G1 and G2. Median OS in non-metastatic G3 was 22.8 months (95\% Cl 20.7-24.9 months).

Metastatic patients of all grades had a median OS of 8.3 months $(95 \% \mathrm{Cl} 7.7-8.8$ months). Poor prognostic factors in univariable analysis of metastatic patients were: male sex, age >65 years, Pan-NEN, L-NEN, O-NEN or U-NEN as primary tumor (compared with GE-NEN), higher tumor grade, higher number of organs with metastases and metastases in the brain, lung, bone and lymph nodes. In multivariable analysis, age $>65$ years, L-NEN, O-NEN or U-NEN as primary tumor, higher tumor grade, higher number of organs with metastases and liver, brain, lung and bone metastases were poor prognostic factors, whereas presence of lymph node metastases was a good prognostic factor (Supplemental Table S9.B).

Metastatic G1 patients had a median OS of 67.4 months (95\% Cl 61.3-73.4 months). In multivariable analysis male sex, age $>65$ years, Pan-NEN, L-NEN or U-NEN as primary tumor (compared with GE-NEN), and liver and brain metastases were poor prognostic factors, whereas pulmonary metastases was a good prognostic factor (Supplemental Table S9.C). Metastatic G2 patients had a median OS of 38.3 months (95\% Cl 32.8-43.7 months). Multivariable analysis revealed age $>65$ years, Pan-NEN, L-NEN or U-NEN as primary tumor (compared with GE-NEN) and brain, lung and lymph nodes metastases as poor prognostic factors (Supplemental Table S9.D). Metastatic G3 patients had a median OS of 3.9 months ( $95 \% \mathrm{Cl} 3.6-4.2$ months) and in multivariable analysis age $>65$ years, L-NEN and U-NEN (compared with GE-NEN), $\geq 2$ organs with metastases and liver metastases were poor prognostic factors. In contrast, Pan-NEN and presence of lymph node metastases were good prognostic factors (Supplemental Table S9.E).

\section{Cohort reference network}

\section{Development of metastatic patterns}

A total of 539 patients were included in the cohort of the reference network, of which 260 (48\%) had metastatic disease at initial presentation (Figure 9.1, Table 9.1, Supplemental Figure S9.A). Metastatic patterns were comparable to the patterns in the nationwide cohort (Supplemental Table S9.F). Patterns of developing metastases were quite similar to patterns seen at diagnosis, but distant lymph node metastases were slightly more frequent than liver metastases ( $12 \%$ and $9 \%$ of all non-metastatic patients at diagnosis) (Supplemental Table S9.G). 


\section{Brain metastases}

Brain metastases were found at initial presentation in 16/103 (16\%) L-NEN patients and in $1 / 41$ (2\%) patient with U-NEN, but not in patients with GE-NEN, Pan-NEN or O-NEN. Although the absolute number of brain metastases was most frequent in G3 L-NEN (i.e. LCNEC, $12 / 54(22 \%))$, relative incidence of brain metastases in this cohort was roughly the same for $\mathrm{G} 2$ (i.e. atypical carcinoid, 2/12 (17\%)). Imaging of the brain with MRIcerebrum was performed at diagnosis in a minority of patients $(1 / 219(0 \%)$ GE-NEN, 2/96 (2\%) Pan-NEN, 23/103 (22\%) L-NEN, 3/80 (4\%) O-NEN and 1/41 (2\%) U-NEN). In almost all patients with L-NEN and brain metastases, the reason to perform a MRIcerebrum was the presence of symptoms indicating brain metastases. In 2/16 L-NEN with brain metastases, patients were asymptomatic and FDG-PET suggested brain metastases. These were confirmed by a MRI-scan. Of patients with metastatic disease but without brain metastases at diagnosis, 10/260 (4\%; 0 GE-NEN, 2 Pan-NEN, 6 L-NEN (all LCNEC), 1 O-NEN and 1 U-NEN)) developed brain metastases during a median follow-up time of 10.1 months (interquartile range 4.2-24.9 months). Therefore, out of 23 metastatic LCNEC without brain metastases at diagnosis, $6(26 \%)$ developed brain metastases during follow-up (Figure 9.3).

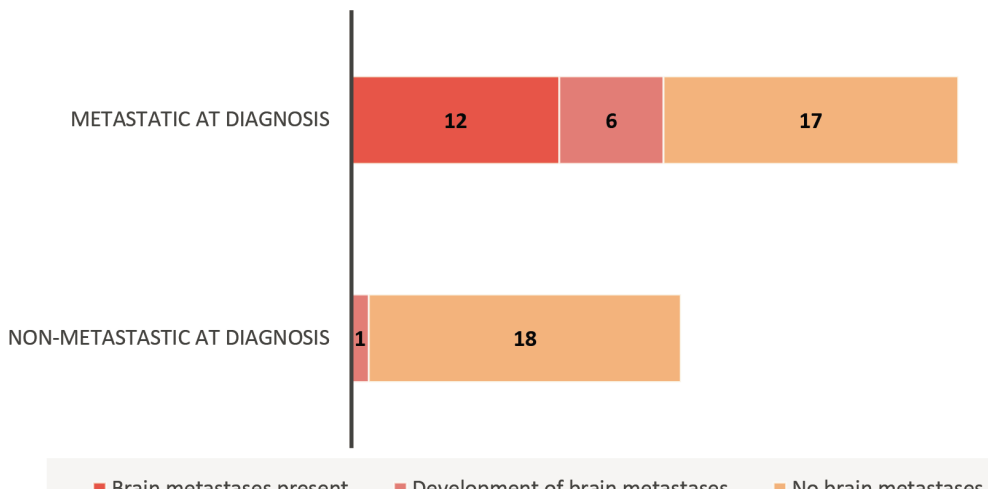

Figure 9.3 Cumulative incidence of brain metastases in large cell neuroendocrine carcinoma of the lung in patients with metastatic disease at diagnosis and non-metastatic disease at diagnosis (cohort reference network). 


\section{Discussion}

A large nationwide retrospective cohort study has been performed in which metastatic patterns of NEN of various primary origins were investigated and additional information on temporal evolution of metastatic spread and diagnostic workup was available for a subcohort of patients from two referral centers. Almost half of the 11,120 NEN patients presented with metastases at diagnosis. In GE- and Pan-NEN, liver was the most prevalent metastatic site at diagnosis, whereas in L-NEN incidence of liver, brain, bone and lung metastases at time of diagnosis was more equal. Remarkably, brain metastases were almost exclusively found in L-NEN.

Besides our data, the presence of metastases at diagnosis in up to $50 \%$ of NEN patients was also found in other series. ${ }^{4-10}$ These studies are in contrast with the study of Riihimaki et al., reporting only $23 \%$ of patients presenting with metastases at diagnosis. ${ }^{11}$ However, in the latter study, only NET were included whereas large cell and small cell NEC were excluded and moreover, distant lymph node metastases and illdefined or unspecific metastatic sites were not reported. ${ }^{11}$ An overview of studies reporting on incidence of metastases in GE-, P- and L-NEN is provided in Table 9.2. , $9-11,22-26$ In general, the metastatic patterns we found were comparable to previous literature. However, to the best of our knowledge, we are the first to include grade 1-3 GE-, P- and L-NEN in one study, herewith providing data for a reliable comparison between the different primary origins. Differences in reported metastatic patterns between studies might be explained by inclusion of solely NET patients (i.e. excluding NEC patients) in some of the studies and diverse definitions used for 'distant' lymph node metastases or 'other' metastases.

Even without routine active screening, brain metastases were found in $14 \%$ of all pulmonary LCNEC patients and in $24 \%$ of patients with stage IV disease in the nationwide cohort. This is in line with previous studies reporting on incidence of brain metastases. ${ }^{9,23}$ Furthermore, in the cohort of the reference network, one out of four patients with stage IV pulmonary LCNEC without brain metastases at diagnosis, developed brain metastases during follow-up, resulting in a cumulative incidence of $51 \%$ in stage IV LCNEC. In our study only 5\% (1/19) of non-stage IV LCNEC patients developed brain metastases during follow-up whereas Zhao et al. found that 35\% $(18 / 52)$ of LCNEC patients treated with curative intent developed brain metastases during follow up, most of them within 2 years after diagnosis. ${ }^{27}$ 
Table 9.2 Overview of current literature on incidences of liver, brain, lung, bone, lymph node, peritoneal and other metastases at diagnosis in gastroenteral, pancreatic and lung neuroendocrine neoplasms.

\begin{tabular}{|c|c|c|c|c|c|c|c|c|c|}
\hline & \multicolumn{2}{|c|}{ Primary tumor } & \multicolumn{7}{|c|}{ Metastases } \\
\hline & Type & $\mathbf{N}$ & $\begin{array}{c}\text { Liver } \\
(\%)\end{array}$ & $\begin{array}{c}\text { Brain } \\
(\%)\end{array}$ & $\begin{array}{c}\text { Lung } \\
(\%)\end{array}$ & $\begin{array}{c}\text { Bone } \\
(\%)\end{array}$ & $\begin{array}{c}\text { Lymph } \\
\text { nodes (\%) }\end{array}$ & $\begin{array}{c}\text { Peritoneal } \\
(\%)\end{array}$ & $\begin{array}{c}\text { Other } \\
(\%)\end{array}$ \\
\hline \multicolumn{10}{|c|}{ Gastroenteral } \\
\hline $\begin{array}{l}\text { Riihimaki } \\
\text { et al. }\end{array}$ & GE-NET & 5,581 & $1,125(20)$ & $37^{1}(1)$ & $95(2)$ & $149(3)$ & - & - & $564^{2}(10)$ \\
\hline $\begin{array}{l}\text { Zheng et } \\
\text { al. }\end{array}$ & GE-NEN & $14,685^{3}$ & $1,459(10)$ & $27(0)$ & $144(1)$ & $115(1)$ & - & - & - \\
\hline $\begin{array}{l}\text { O'Conner } \\
\text { et al. }\end{array}$ & GE-NEN & 270 & $114(42)$ & $0(0)$ & $1(0)$ & $1(0)$ & $101^{4}(37)$ & $36(13)$ & - \\
\hline Chen et al. & SI-NEN & 277 & 52 (19) & $1(0)$ & $1(0)$ & $3(1)$ & - & - & $13(5)$ \\
\hline $\begin{array}{l}\text { Madani et } \\
\text { al. }\end{array}$ & GE-NEN ${ }^{5}$ & 3,413 & - & - & - & - & - & $213(6)$ & - \\
\hline \multicolumn{10}{|l|}{ Pancreas } \\
\hline $\begin{array}{l}\text { Riihimaki } \\
\text { et al. }\end{array}$ & Pan-NET ${ }^{6}$ & 275 & $148(54)$ & $3^{1}(1)$ & $12(4)$ & $28(10)$ & - & - & $44^{2}(16)$ \\
\hline $\begin{array}{l}\text { Wang et } \\
\text { al. }\end{array}$ & Pan-NEN & 3,909 & $1,133^{7}(29)$ & $5^{7}(0)$ & $28^{7}(1)$ & $21^{7}(6)$ & - & - & - \\
\hline $\begin{array}{l}\text { O'Conner } \\
\text { et al. }\end{array}$ & Pan-NEN & 116 & $74(64)$ & $1(1)$ & $4(3)$ & $2(2)$ & $44^{4}(38)$ & $5(4)$ & - \\
\hline $\begin{array}{l}\text { Cetinyaka } \\
\text { et al. }\end{array}$ & Pan-NET & 114 & $49(43)$ & - & - & - & - & - & - \\
\hline $\begin{array}{l}\text { Madani et } \\
\text { al. } \\
\text { Lung }\end{array}$ & Pan-NEN ${ }^{5}$ & 701 & - & - & - & - & - & $21(3)$ & - \\
\hline $\begin{array}{l}\text { Riihimaki } \\
\text { et al. }\end{array}$ & L-NET & 1,113 & $116(10)$ & $54(5)$ & $43(4)$ & 74 (7) & - & - & $86^{2}(8)$ \\
\hline $\begin{array}{l}\text { Kinslow et } \\
\text { al. }\end{array}$ & LCNEC & 1,681 & 323 (19) & 322 (19) & $188(11)$ & 294 (17) & - & - & - \\
\hline $\begin{array}{l}\text { Derks et } \\
\text { al. }\end{array}$ & LCNEC $^{5}$ & 383 & $47 \%^{8}$ & $23 \%^{8}$ & $14 \%^{8}$ & $32 \%^{8}$ & $16 \%^{8}$ & - & - \\
\hline
\end{tabular}

${ }^{1}$ Total nervous system included; ${ }^{2}$ Including metastases of pleura/mediastinum, 'other' intra-abdominal (=nonliver) metastases, and 'other' metastases in general; ${ }^{3}$ Remarkably high number of grade I tumors $(7,387 / 10,107$ with known grade $(73 \%))$ and low number of grade III/IV tumors $(1,108 / 10,107$ (11\%)), reason unknown; ${ }^{4}$ Might also include non-distant lymph nodes; ${ }^{5}$ Partly overlapping with our nationwide cohort; ${ }^{6}$ Liver, gall and pancreas neuroendocrine tumors included; ${ }^{7} 198$ patients (5\%) had multiple metastases. Those are not included in numbers of liver, brain, bone and lung metastases; ${ }^{8}$ Exact numbers not available. Abbreviations: GE = gastroenteral; NET = neuroendocrine tumor; NEN = neuroendocrine neoplasm; $\mathrm{SI}=\mathrm{small}$ intestine; Pan = pancreas; $L=$ lung; $L C N E C=$ large cell neuroendocrine carcinoma (pulmonary).

The low percentage of development of brain metastases we found in non-metastatic LCNEC at diagnosis might be due to the short follow-up time. Therefore, considering the substantial numbers of brain metastases found in pulmonary LCNEC, screening for brain metastases may be considered in these patients to improve treatment management. For patients initially diagnosed with stage I-III LCNEC, presence of 
asymptomatic brain metastases will result in palliative treatment instead of more aggressive treatment with curative intention. For LCNEC patients already diagnosed with metastases, presence of additional brain metastases might influence treatment management. No large sets of data are available on effectivity of systemic therapy for brain metastases in LCNEC. However, based on experience from NSCLC and the fact that some LCNEC can present with targetable mutations, it might be reasonable to perform mutational analysis in LCNEC patients and especially those with brain metastases and treat selected patients with new generation TKIs instead of chemotherapy. ${ }^{28-32}$ The prevalence of brain metastases in $3 \%$ of atypical carcinoids might be an underestimation since screening was not performed in this cohort. Therefore, the actual number of patients could even be higher, which might also justify screening in this NEN subgroup.

So far, only limited evidence about the prognosis of NEN of different primary origin and with specific patterns of metastatic spread has been reported. Improved survival has been observed in patients with liver metastases, compared to patients with brain, bone or lung metastases in GE-NEN. ${ }^{26}$ However, in our cohort presence of liver metastases was a poor prognostic factor. Unfavorable survival has been reported for NEN patients with bone metastases, as is confirmed by our data. ${ }^{5,33,34}$ We found a lower survival in metastatic L-NEN, O-NEN and U-NEN compared to gastroenteral primary origin, as was described for unknown primary origin by Riihimaki et al. ${ }^{11}$ On the other hand, based on our results, Pan-NEN was only a poor prognostic factor in G1 and G2 whereas it seems to be a good prognostic factor in G3 NEN. Taken together, both primary tumor origin and metastatic patterns appear to be inconsistent as prognostic indicators and therefore prognosis can better be predicted by robust factors as age, tumor grade and number of organs with metastatic lesions.

Despite the fact that we could get inside in the temporal evolution of patterns of metastatic spread in NEN from different primary origins for patients in our reference network, the median follow-up time was limited (15 months). Therefore, only an indication of development of metastases during the first years after diagnosis could be provided, whereas especially for low- and intermediate-grade tumors, metastases might develop years after initial presentation. Another limitation of this study is the retrospective design. For the comprehensive large nationwide cohort only limited variables are available and for example, development of metastases during follow-up and WHO performance score are not registered in this database. In the cohort of our reference network, we could obtain most of the data from medical records for the last five years. However, the number of patients in the two centers was limited and some 
data was missing due to follow-up outside the referral centers. The slight differences between the two cohorts might be explained by the fact that patients of two NENreferral centers were included in the latter one, whereas a non-selected population was used for the nationwide cohort. Maybe patients with non-metastatic G1 or G2 NEN are not consequently referred to one of our centers. The same might apply to patients with G3 NEC and a very poor prognosis.

In conclusion, our data show that nearly half of the patients with NEN present with metastatic disease at initial diagnosis. In GE- and Pan-NEN liver metastases are most common, whereas in L-NEN incidence of liver metastases is less frequent and incidence of brain, lung and bone metastases is more equal. Interestingly, brain metastases are almost exclusively observed in L-NEN, and more than half of pulmonary LCNEC patients with metastases at diagnosis have brain metastases at initial presentation or develop brain metastases during follow-up. Therefore, screening for brain metastases might be considered in metastatic LCNEC and other L-NEN which may impact treatment management. Since brain metastases were very rare in GE-, P- and O-NEN, screening does not seem useful in these subtypes.

\section{Acknowledgement}

We thank the registration team of the Netherlands Comprehensive Cancer Organisation (IKNL) for the collection of data for the Netherlands Cancer Registry as well as IKNL staff for scientific advice. We thank A. Willems, M. Bilochvostenko, S. Bayazit and S. Bruurmijn-Verhoeven for their help with obtaining data from medical records. 


\section{References}

1. Lloyd RV, Osamura RY, Kloppel G, et al. WHO classification of Tumours of Endocrine Organs. Lyon: International Agency for Research on Cancer; 2017.

2. Travis WD, Brambilla E, Burke AP, et al. WHO Classification of Tumours of the Lung, Pleura, Thymus and Heart Lyon: International Agency for Research on Cancer, 4th edition, March 2015; 2015.

3. WHO. WHO classification of Tumours, Digestive System Tumours, 5th edition. WHO; 2019.

4. Darba J, Marsa A. Exploring the current status of neuroendocrine tumours: a population-based analysis of epidemiology, management and use of resources. BMC Cancer 2019;19:1226.

5. Scharf M, Petry V, Daniel H, et al. Bone Metastases in Patients with Neuroendocrine Neoplasm: Frequency and Clinical, Therapeutic, and Prognostic Relevance. Neuroendocrinology 2018;106:30-37.

6. Liu Y, Ye S, Zhu Y, et al. Impact of tumour size on metastasis and survival in patients with pancreatic neuroendocrine tumours (PNETs): A population based study. J Cancer 2019;10:6349-6357.

7. Boyar Cetinkaya R, Vatn M, Aabakken L, et al. Survival and prognostic factors in well-differentiated pancreatic neuroendocrine tumors. Scandinavian journal of gastroenterology 2014;49:734-741.

8. Wang J, Ye L, Cai H, et al. Comparative study of large cell neuroendocrine carcinoma and small cell lung carcinoma in high-grade neuroendocrine tumors of the lung: a large population-based study. $J$ Cancer 2019;10:4226-4236.

9. Kinslow CJ, May MS, Saqi A, et al. Large-Cell Neuroendocrine Carcinoma of the Lung: A PopulationBased Study. Clin Lung Cancer 2020;21(2):e99-r113.

10. Wang S, Zhang J, Liu S, et al. The prognostic analysis of different metastatic patterns in pancreatic neuroendocrine tumors patients: A population based analysis. Medicine (Baltimore) 2019;98:e17773.

11. Riihimaki $M$, Hemminki $A$, Sundquist $K$, et al. The epidemiology of metastases in neuroendocrine tumors. Int J Cancer 2016;139:2679-2686.

12. Krug $S$, Teupe $F$, Michl $P$, et al. Brain metastases in patients with neuroendocrine neoplasms: risk factors and outcome. BMC Cancer 2019;19:362.

13. Hoberück S, Michler E, Zöphel K, et al. Brain Metastases of a Neuroendocrine Tumor Visualized by 68GaDOTATATE PET/CT. Clinical nuclear medicine 2019;44:50-52.

14. Seute $T$, Leffers $P$, ten Velde GP, et al. Neurologic disorders in 432 consecutive patients with small cell lung carcinoma. Cancer 2004;100:801-806.

15. Fruh M, De Ruysscher D, Popat S, et al. Small-cell lung cancer (SCLC): ESMO Clinical Practice Guidelines for diagnosis, treatment and follow-up. Ann Oncol 2013;24 Suppl 6:vi99-105.

16. Visser O, Siesling S, Dijk Jv, et al. Incidence of cancer in the Netherlands 1999/2000: Eleventh report of the Netherlands Cancer Registry. 2003 Available at https://www.scienceopen.com/document?vid= 7c2ca696-b8cc-425a-bf17-a4c97974869b.

17. Korse CM, Taal BG, van Velthuysen ML, et al. Incidence and survival of neuroendocrine tumours in the Netherlands according to histological grade: experience of two decades of cancer registry. Eur J Cancer 2013;49:1975-1983.

18. Falconi M, Eriksson B, Kaltsas G, et al. ENETS Consensus Guidelines Update for the Management of Patients with Functional Pancreatic Neuroendocrine Tumors and Non-Functional Pancreatic Neuroendocrine Tumors. Neuroendocrinology 2016;103:153-171.

19. Garcia-Carbonero R, Sorbye H, Baudin E, et al. ENETS Consensus Guidelines for High-Grade Gastroenteropancreatic Neuroendocrine Tumors and Neuroendocrine Carcinomas. Neuroendocrinology 2016;103:186-194.

20. Niederle B, Pape UF, Costa F, et al. ENETS Consensus Guidelines Update for Neuroendocrine Neoplasms of the Jejunum and lleum. Neuroendocrinology 2016;103:125-138.

21. Oberg K, Hellman P, Ferolla P, et al. Neuroendocrine bronchial and thymic tumors: ESMO Clinical Practice Guidelines for diagnosis, treatment and follow-up. Ann Oncol 2012;23 Suppl 7:vii120-123.

22. Chen $L$, Zhou L, Zhang M, et al. Clinicopathological features and prognostic validity of WHO grading classification of SI-NENs. BMC Cancer 2017;17:521.

23. Derks JL, Hendriks LE, Buikhuisen WA, et al. Clinical features of large cell neuroendocrine carcinoma: a population-based overview. Eur Respir J 2016;47:615-624. 
24. Madani A, Thomassen I, van Gestel Y, et al. Peritoneal Metastases from Gastroenteropancreatic Neuroendocrine Tumors: Incidence, Risk Factors and Prognosis. Annals of surgical oncology 2017;24:2199-2205.

25. O'Connor JM, Marmissolle F, Bestani C, et al. Observational study of patients with gastroenteropancreatic and bronchial neuroendocrine tumors in Argentina: Results from the large database of a multidisciplinary group clinical multicenter study. Mol Clin Oncol 2014;2:673-684.

26. Zheng Z, Chen C, Jiang $L$, et al. Incidence and risk factors of gastrointestinal neuroendocrine neoplasm metastasis in liver, lung, bone, and brain: A population-based study. Cancer Med 2019;8:7288-7298.

27. Zhao $Y$, Castonguay M, Wilke D, et al. Treatment outcomes and incidence of brain metastases in pulmonary large cell neuroendocrine carcinoma. Current problems in cancer 2019;43:54-65.

28. Inno A, Di Noia V, D'Argento E, et al. State of the art of chemotherapy for the treatment of central nervous system metastases from non-small cell lung cancer. Translational lung cancer research 2016;5:599-609.

29. Zimmermann S, Dziadziuszko R, Peters S. Indications and limitations of chemotherapy and targeted agents in non-small cell lung cancer brain metastases. Cancer treatment reviews 2014;40:716-722.

30. Abdallah SM, Wong A. Brain metastases in non-small-cell lung cancer: are tyrosine kinase inhibitors and checkpoint inhibitors now viable options? Current oncology (Toronto, Ont) 2018;25:S103-s114.

31. George J, Walter V, Peifer M, et al. Integrative genomic profiling of large-cell neuroendocrine carcinomas reveals distinct subtypes of high-grade neuroendocrine lung tumors. Nat Commun 2018;9:1048.

32. Rekhtman N, Pietanza MC, Hellmann MD, et al. Next-Generation Sequencing of Pulmonary Large Cell Neuroendocrine Carcinoma Reveals Small Cell Carcinoma-like and Non-Small Cell Carcinoma-like Subsets. Clin Cancer Res 2016;22:3618-3629.

33. Kavecansky J, Wei L, Caronia L, et al. Bone metastases in well-to-moderately differentiated neuroendocrine tumors: a single institutional review from the Ohio State University Medical Center. Pancreas 2015;44:198-203.

34. Lee L, Igarashi $\mathrm{H}$, Fujimori $\mathrm{N}$, et al. Long-term outcomes and prognostic factors in 78 Japanese patients with advanced pancreatic neuroendocrine neoplasms: a single-center retrospective study. Jpn J Clin Oncol 2015;45:1131-1138. 
Supplemental material
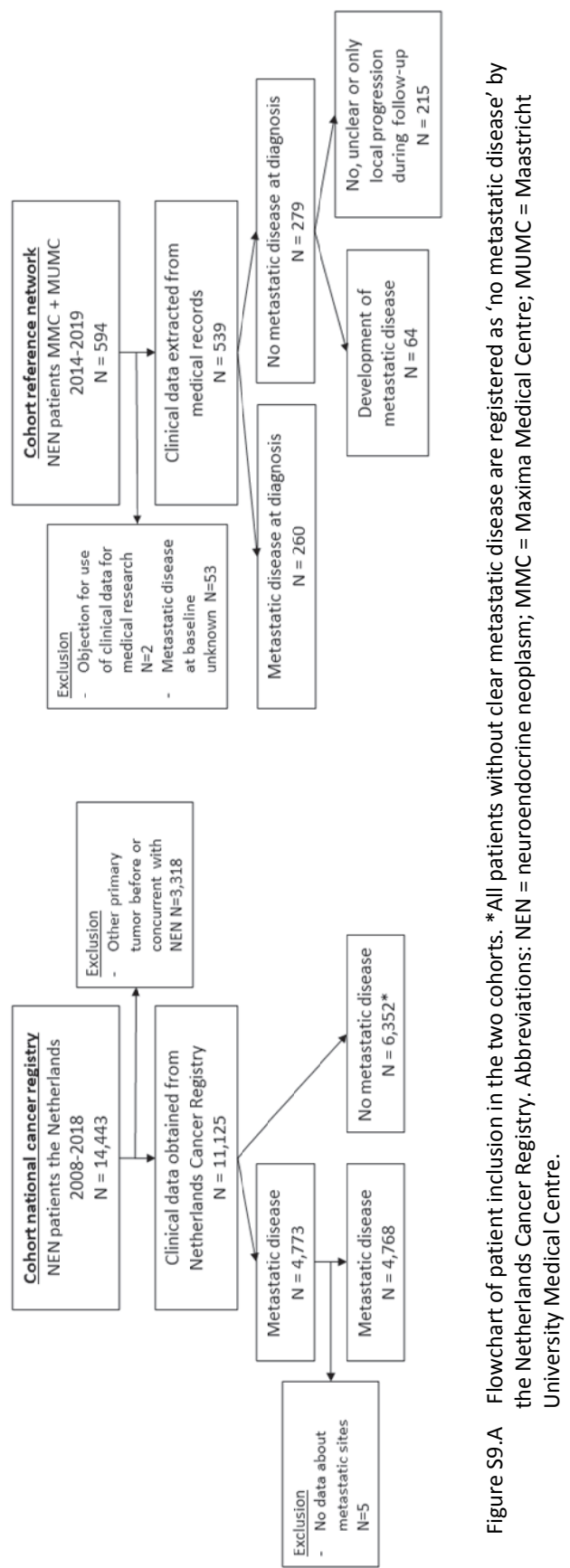


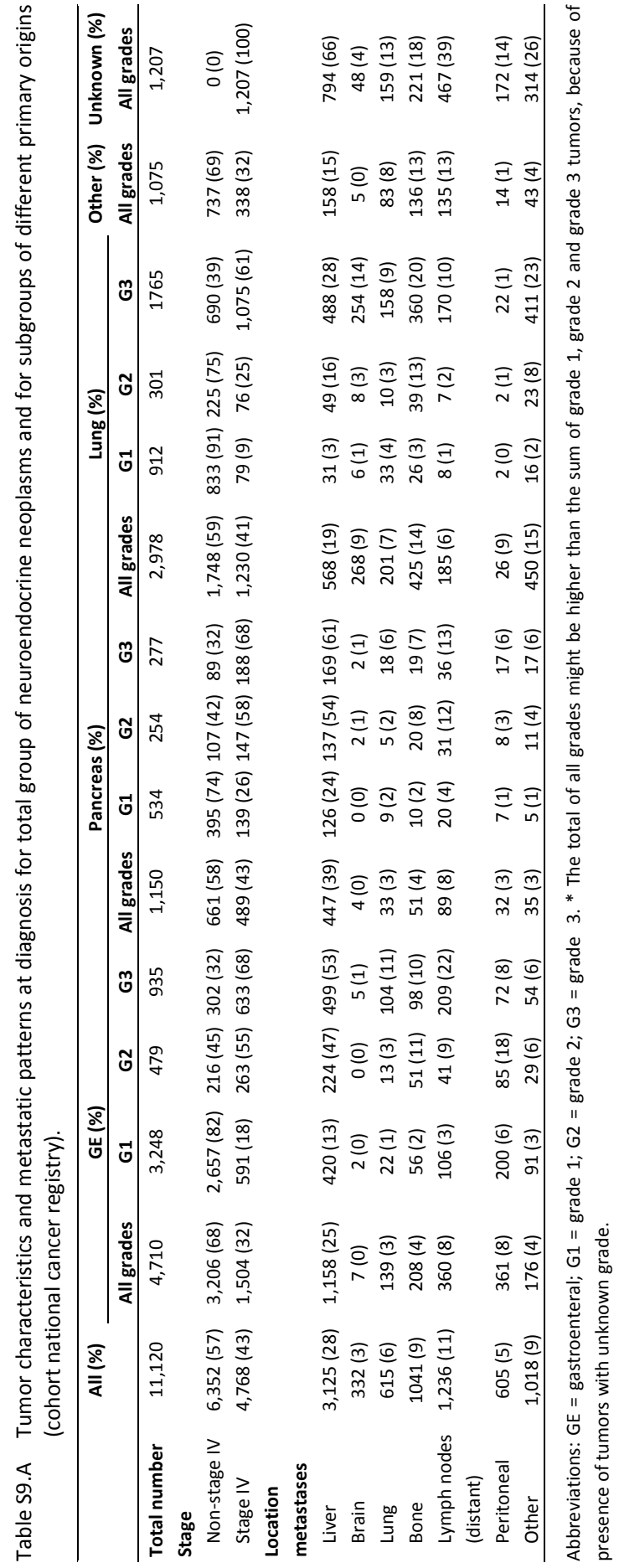


Table S9.B Prognostic factors for overall survival in patients with metastatic neuroendocrine neoplasms (cohort national cancer registry).

\begin{tabular}{|c|c|c|c|c|c|}
\hline & $\mathbf{N}$ & $\begin{array}{l}\text { Univariable } \\
\text { HR }(95 \% \mathrm{Cl})\end{array}$ & p-value & $\begin{array}{c}\text { Multivariable } \\
\text { HR }(95 \% \mathrm{Cl})\end{array}$ & p-value \\
\hline \multicolumn{6}{|l|}{ Gender } \\
\hline Male & 2,588 & Ref. & & Ref. & \\
\hline Female & 2,157 & $0.90(0.84-0.96)$ & 0.001 & $0.97(0.91-1.04)$ & 0.39 \\
\hline \multicolumn{6}{|l|}{ Age } \\
\hline$\leq 65$ & 2,187 & Ref. & & Ref. & \\
\hline$>65$ & 2,558 & $1.66(1.56-1.77)$ & $<0.001$ & $1.64(1.53-1.75)$ & $<0.001$ \\
\hline \multicolumn{6}{|l|}{ Primary tumor } \\
\hline GE & 1,499 & Ref. & & Ref. & \\
\hline Pancreas & 488 & $1.18(1.04-1.33)$ & 0.009 & $1.06(0.93-1.19)$ & 0.40 \\
\hline Lung & 1,220 & $2.68(2.46-2.93)$ & $<0.001$ & $1.53(1.39-1.69)$ & $<0.001$ \\
\hline Other & 335 & $2.41(2.12-2.73)$ & $<0.001$ & $1.18(1.03-1.36)$ & 0.015 \\
\hline Unknown & 1,203 & $1.86(1.70-2.03)$ & $<0.001$ & $1.39(1.27-1.52)$ & $<0.001$ \\
\hline \multicolumn{6}{|l|}{ Tumor grade } \\
\hline Grade 1 & 1,123 & Ref. & & Ref. & \\
\hline Grade 2 & 604 & $1.32(1.15-1.51)$ & $<0.001$ & $1.24(1.09-1.43)$ & 0.002 \\
\hline Grade 3 & 2,985 & $5.50(5.01-6.04)$ & $<0.001$ & $5.38(4.87-5.95)$ & $<0.001$ \\
\hline \multicolumn{6}{|l|}{$\begin{array}{l}\text { Number of organs with } \\
\text { metastases }\end{array}$} \\
\hline 1 & 2,550 & Ref. & & Ref. & \\
\hline $2-3$ & 2,055 & $1.40(1.31-1.50)$ & $<0.001$ & $1.21(1.11-1.32)$ & $<0.001$ \\
\hline$\geq 4$ & 140 & $1.69(1.40-2.03)$ & $<0.001$ & $1.29(1.01-1.66)$ & 0.042 \\
\hline \multicolumn{6}{|l|}{ Location metastases } \\
\hline Liver & 3,110 & $1.03(0.96-1.10)$ & 0.44 & $1.69(1.51-1.89)$ & $<0.001$ \\
\hline Brain & 328 & $1.63(1.45-1.83)$ & $<0.001$ & $1.09(0.94-1.26)$ & 0.026 \\
\hline Lung & 610 & $1.53(1.40-1.68)$ & $<0.001$ & $1.19(1.07-1.32)$ & 0.001 \\
\hline Bone & 1,034 & $1.44(1.33-1.55)$ & $<0.001$ & $1.24(1.08-1.42)$ & 0.002 \\
\hline Lymph nodes (distant) & 1,233 & $1.13(1.05-1.22)$ & $<0.001$ & $0.84(0.74-0.96)$ & 0.011 \\
\hline Liver*Bone $^{1}$ & - & - & - & $0.77(0.65-0.90)$ & 0.001 \\
\hline Liver*Lymph nodes ${ }^{1}$ & - & - & - & $1.17(1.00-1.37)$ & 0.053 \\
\hline Brain*Lymph nodes ${ }^{1}$ & - & - & - & $1.06(0.74-1.50)$ & 0.77 \\
\hline
\end{tabular}

Abbreviations: $\mathrm{HR}=$ Hazard Ratio; $95 \% \mathrm{Cl}=95 \%$ Confidence Interval; Ref. = Reference; $\mathrm{GE}=$ gastroenteral. ${ }^{1}$ Interaction terms included in the final multivariable model. Events $=3,787$. 
Table S9.C Prognostic factors for overall survival in patients with metastatic grade 1 neuroendocrine neoplasms (cohort national cancer registry).

\begin{tabular}{|c|c|c|c|c|c|}
\hline & $\mathbf{N}$ & $\begin{array}{l}\text { Univariable } \\
\text { HR }(95 \% \mathrm{Cl})\end{array}$ & p-value & $\begin{array}{c}\text { Multivariable } \\
\text { HR }(95 \% \mathrm{Cl})\end{array}$ & p-value \\
\hline \multicolumn{6}{|l|}{ Gender } \\
\hline Male & 571 & Ref. & & Ref. & \\
\hline Female & 552 & $0.85(0.72-1.00)$ & 0.047 & $0.83(0.70-0.97)$ & 0.022 \\
\hline \multicolumn{6}{|l|}{ Age } \\
\hline$\leq 65$ & 582 & Ref. & & Ref. & \\
\hline$>65$ & 541 & $2.14(1.82-2.52)$ & $<0.001$ & $2.24(1.90-2.65)$ & $<0.001$ \\
\hline \multicolumn{6}{|l|}{ Primary tumor } \\
\hline $\mathrm{GE}$ & 588 & Ref. & & Ref. & \\
\hline Pancreas & 138 & $1.64(1.28-2.09)$ & $<0.001$ & $1.73(1.35-2.23)$ & $<0.001$ \\
\hline Lung & 77 & $2.01(1.50-2.87)$ & $<0.001$ & $2.45(1.70-3.53)$ & $<0.001$ \\
\hline Other & 10 & $1.18(0.49-2.87)$ & 0.71 & $0.97(0.40-2.38)$ & 0.95 \\
\hline Unknown & 310 & $2.04(1.70-2.45)$ & $<0.001$ & $1.89(1.57-2.29)$ & $<0.001$ \\
\hline \multicolumn{6}{|l|}{$\begin{array}{l}\text { Number of organs with } \\
\text { metastases }\end{array}$} \\
\hline 1 & 683 & Ref. & & Ref. & \\
\hline $2-3$ & 421 & $1.55(1.32-1.82)$ & $<0.001$ & $1.19(0.96-1.47)$ & 0.12 \\
\hline$\geq 4$ & 19 & $1.46(0.72-2.95)$ & 0.292 & $0.49(0.20-1.19)$ & 0.11 \\
\hline \multicolumn{6}{|l|}{ Location metastases } \\
\hline Liver & 814 & $1.61(1.32-1.96)$ & $<0.001$ & $1.50(1.19-1.89)$ & 0.001 \\
\hline Brain & 12 & $2.32(1.24-4.34)$ & 0.008 & $2.36(1.20-4.65)$ & 0.013 \\
\hline Lung & 95 & $1.30(0.98-1.72)$ & 0.066 & $0.55(0.31-0.96)$ & 0.035 \\
\hline Bone & 130 & $1.47(1.15-1.87)$ & 0.002 & $1.22(0.90-1.64)$ & 0.20 \\
\hline Lymph nodes (distant) & 233 & $1.22(1.00-1.48)$ & 0.045 & $1.05(0.83-1.34)$ & 0.67 \\
\hline${\text { Liver* } \text { Lung }^{1}}$ & & - & - & $3.02(1.57-5.79)$ & 0.001 \\
\hline Lung*Bone $^{1}$ & & - & - & $3.13(1.47-6.65)$ & 0.003 \\
\hline
\end{tabular}

Abbreviations: $\mathrm{HR}=$ Hazard Ratio; $95 \% \mathrm{Cl}=95 \%$ Confidence Interval; Ref. = Reference; GE = gastroenteral. ${ }^{1}$ Interaction terms included in the final multivariable model. Events $=601$. 
Table S9.D Prognostic factors for overall survival in patients with metastatic grade 2 neuroendocrine neoplasms (cohort national cancer registry).

\begin{tabular}{|c|c|c|c|c|c|}
\hline & $\mathbf{N}$ & $\begin{array}{l}\text { Univariable } \\
\text { HR }(95 \% \mathrm{Cl})\end{array}$ & p-value & $\begin{array}{c}\text { Multivariable } \\
\text { HR }(95 \% \mathrm{CI})\end{array}$ & p-value \\
\hline \multicolumn{6}{|l|}{ Gender } \\
\hline Male & 312 & Ref. & & & \\
\hline Female & 292 & $1.14(0.92-1.41)$ & 0.25 & - & - \\
\hline \multicolumn{6}{|l|}{ Age } \\
\hline$\leq 65$ & 314 & Ref. & & Ref. & \\
\hline$>65$ & 290 & $1.73(1.40-2.15)$ & $<0.001$ & $2.00(1.60-2.49)$ & $<0.001$ \\
\hline \multicolumn{6}{|l|}{ Primary tumor } \\
\hline $\mathrm{GE}$ & 263 & Ref. & & Ref. & \\
\hline Pancreas & 147 & $2.01(1.52-2.66)$ & $<0.001$ & $2.28(1.70-3.06)$ & $<0.001$ \\
\hline Lung & 74 & $3.58(2.59-4.96)$ & $<0.001$ & $3.87(2.73-5.50)$ & $<0.001$ \\
\hline Other & 11 & $2.14(1.11-4.11)$ & 0.023 & $1.52(0.75-3.08)$ & 0.246 \\
\hline Unknown & 109 & $1.79(1.31-2.43)$ & $<0.001$ & $1.74(1.26-2.39)$ & 0.001 \\
\hline \multicolumn{6}{|l|}{$\begin{array}{l}\text { Number of organs with } \\
\text { metastases }\end{array}$} \\
\hline 1 & 320 & Ref. & & Ref. & \\
\hline $2-3$ & 260 & $1.15(0.92-1.43)$ & 0.21 & $0.84(0.61-1.17)$ & 0.31 \\
\hline$\geq 4$ & 24 & $1.59(0.94-2.70)$ & 0.087 & $1.16(0.56-2.40)$ & 0.69 \\
\hline \multicolumn{6}{|l|}{ Location metastases } \\
\hline Liver & 503 & $1.12(0.83-1.51)$ & 0.45 & $1.41(1.00-1.99)$ & 0.051 \\
\hline Brain & 12 & $3.07(1.63-5.78)$ & 0.001 & $2.54(1.24-5.21)$ & 0.011 \\
\hline Lung & 38 & $1.47(0.98-2.21)$ & 0.061 & $3.41(1.87-6.20)$ & $<0.001$ \\
\hline Bone & 138 & $1.33(1.04-1.70)$ & 0.024 & $1.30(0.91-1.84)$ & 0.15 \\
\hline Lymph nodes (distant) & 119 & $1.22(0.94-1.59)$ & 0.13 & $1.52(1.06-2.18)$ & 0.022 \\
\hline Lung*Bone $^{1}$ & & - & - & $0.29(0.13-0.68)$ & 0.004 \\
\hline Lung $^{*}$ Lymph nodes ${ }^{1}$ & & - & - & $0.29(0.08-1.02)$ & 0.053 \\
\hline
\end{tabular}

Abbreviations: $\mathrm{HR}=$ Hazard Ratio; $95 \% \mathrm{Cl}=$ 95\% Confidence Interval; Ref. = Reference; $\mathrm{GE}=$ gastroenteral. ${ }^{1}$ Interaction terms included in the final multivariable model. Events $=335$. 
Table S9.E Prognostic factors for overall survival in patients with metastatic grade 3 neuroendocrine neoplasms (cohort national cancer registry).

\begin{tabular}{|c|c|c|c|c|c|}
\hline & $\mathbf{N}$ & $\begin{array}{l}\text { Univariable } \\
\text { HR }(95 \% \mathrm{Cl}) \\
\end{array}$ & p-value & $\begin{array}{c}\text { Multivariable } \\
\text { HR }(95 \% \mathrm{Cl})\end{array}$ & p-value \\
\hline \multicolumn{6}{|l|}{ Gender } \\
\hline Male & 1,689 & Ref. & & & \\
\hline Female & 1,296 & $0.98(0.91-1.06)$ & 0.64 & - & - \\
\hline \multicolumn{6}{|l|}{ Age } \\
\hline$\leq 65$ & 1,271 & Ref. & & Ref. & \\
\hline$>65$ & 1,714 & $1.48(1.38-1.60)$ & $<0.001$ & $1.53(1.42-1.65)$ & $<0.001$ \\
\hline \multicolumn{6}{|l|}{ Primary tumor } \\
\hline $\mathrm{GE}$ & 631 & Ref. & & Ref. & \\
\hline Pancreas & 188 & $0.76(0.64-0.90)$ & 0.002 & $0.72(0.61-0.86)$ & $<0.001$ \\
\hline Lung & 1,069 & $1.05(0.95-1.16)$ & 0.33 & $1.22(1.09-1.36)$ & 0.001 \\
\hline Other & 314 & $0.88(0.77-1.01)$ & 0.077 & $1.00(0.87-1.16)$ & 0.99 \\
\hline Unknown & 783 & $1.05(0.94-1.17)$ & 0.38 & $1.16(1.04-1.30)$ & 0.009 \\
\hline \multicolumn{6}{|l|}{$\begin{array}{l}\text { Number of organs with } \\
\text { metastases }\end{array}$} \\
\hline 1 & 1,527 & Ref. & & Ref. & \\
\hline $2-3$ & 1,362 & $1.39(1.29-1.50)$ & $<0.001$ & $1.29(1.16-1.43)$ & $<0.001$ \\
\hline$\geq 4$ & 96 & $1.76(1.43-2.17)$ & $<0.001$ & $1.60(1.22-2.11)$ & 0.001 \\
\hline \multicolumn{6}{|l|}{ Location metastases } \\
\hline Liver & 1,763 & $1.53(1.42-1.65)$ & $<0.001$ & $1.64(1.46-1.84)$ & $<0.001$ \\
\hline Brain & 304 & $0.91(0.81-1.03)$ & 0.13 & $1.02(0.88-1.18)$ & 0.79 \\
\hline Lung & 476 & $1.18(1.07-1.31)$ & 0.001 & $1.07(0.96-1.20)$ & 0.24 \\
\hline Bone & 761 & $1.12(1.03-1.22)$ & 0.007 & $1.16(1.00-1.34)$ & 0.051 \\
\hline Lymph nodes (distant) & 874 & $0.86(0.80-0.93)$ & $<0.001$ & $0.83(0.75-0.92)$ & 0.001 \\
\hline Liver*Bone $^{1}$ & & - & - & $0.75(0.63-0.90)$ & 0.002 \\
\hline
\end{tabular}

Abbreviations: $\mathrm{HR}=$ Hazard Ratio; $95 \% \mathrm{Cl}=95 \%$ Confidence Interval; Ref. = Reference; GE = gastroenteral. ${ }^{1}$ Interaction terms included in the final multivariable model. Events $=2,842$. 


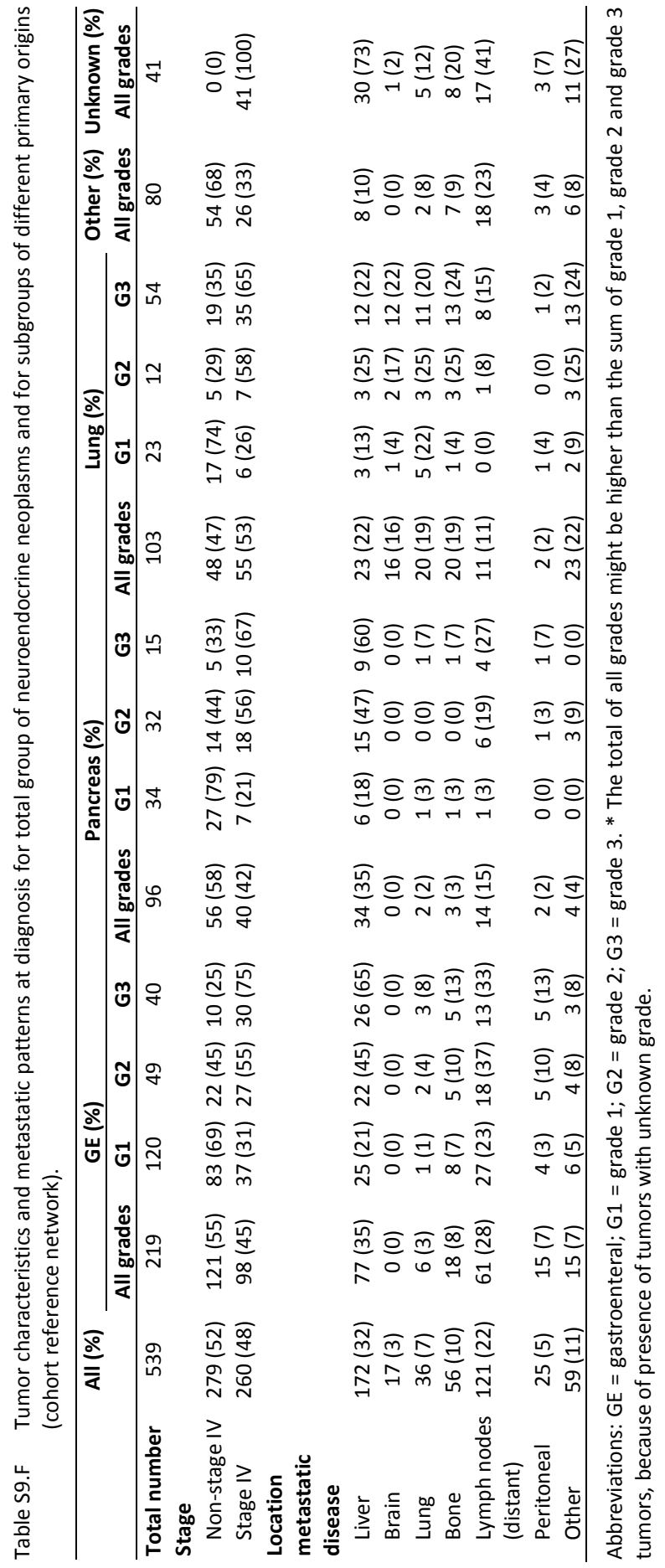


Table S9.G Development of metastatic disease after initial presentation with non-metastatic neuroendocrine neoplasms for different primary origins (cohort reference network).

\begin{tabular}{lcccccc}
\hline & All (\%) & GE (\%) & Pancreas (\%) & Lung (\%) & Other (\%) & Unknown (\%) \\
\hline $\begin{array}{l}\text { Non-metastatic at } \\
\text { diagnosis }\end{array}$ & 279 & 121 & 56 & 48 & 54 & 0 \\
$\begin{array}{l}\text { New metastases } \\
\text { Location metastatic }\end{array}$ & $64(23)$ & $19(16)$ & $9(16)$ & $12(25)$ & $24(44)$ & 0 \\
disease & & & & & \\
$\quad$ Liver & $25(9)$ & $8(7)$ & $7(13)$ & $5(10)$ & $5(9)$ & 0 \\
Brain & $3(1)$ & $0(0)$ & $0(0)$ & $1(2)$ & $2(4)$ & 0 \\
Lung & $8(3)$ & $2(2)$ & $0(0)$ & $3(6)$ & $3(6)$ & 0 \\
Bone & $20(7)$ & $9(7)$ & $0(0)$ & $4(8)$ & $8(15)$ & 0 \\
Lymph nodes (distant) & $33(12)$ & $13(11)$ & $4(7)$ & $2(4)$ & $14(26)$ & 0 \\
Peritoneal & $6(2)$ & $4(3)$ & $1(2)$ & $0(0)$ & $1(2)$ & 0 \\
$\quad$ Other & $10(4)$ & $2(2)$ & $1(2)$ & $1(2)$ & $6(11)$ & 0 \\
\hline
\end{tabular}

Abbreviations: GE = gastroenteral. 



\section{Chapter 10}

General discussion 



\section{General discussion}

Large cell neuroendocrine carcinoma (LCNEC) is a rare disease constituting $1-3 \%$ of patients diagnosed with lung carcinoma. ${ }^{1-5}$ Despite presentation with metastatic disease in about half of the patients and an aggressive course of disease resulting in low survival rates, treatment regimens have not evolved significantly in the last decades and evidence based guidelines are lacking. ${ }^{6-8}$ Recent research has shown that LCNEC, although classified by the world health organization (WHO) as a single entity, maybe more heterogenous with regard to both its molecular and clinical characteristics. ${ }^{9-16}$ Two molecular LCNEC subtypes have been identified: a small cell lung carcinoma (SCLC)-like subtype with TP53 and RB1 mutations and a non-small cell lung carcinoma (NSCLC)-like subtype, enriched for mutations in TP53, KEAP1, STK11 and KRAS. The potential relevance of this subclassification is strengthened by the findings that these two LCNEC subtypes might have a different response to chemotherapeutic regimens. ${ }^{9,17}$ In this thesis we aimed to further unravel the heterogeneity of LCNEC by an in depth evaluation of patients presenting with LCNEC having unique clinical and/or pathological characteristics. Furthermore, we evaluated possible predictive and prognostic markers in clinical and molecular subtypes of LCNEC.

Imaging characteristics might be helpful in classification of lung cancer subtypes, e.g. SCLC is more often located central in the lungs, whereas NSCLC is more often located peripheral. $^{18-23}$ Hence, the radiological presentation of LCNEC maybe utilized to improve (sub)classification of LCNEC. In chapter 2, we evaluated the potential of imaging characteristics and a radiomics signature to identify SCLC, NSCLC and molecular subtypes of LCNEC. To further improve our understanding of LCNEC with a NSCLC-like molecular subtype, in chapter $\mathbf{3}$ we aimed to identify commonly activated molecular pathways in LCNEC whom are closely related to adenocarcinomas (NSCLC) based on histopathology. Therefore, we profiled tumors of patients presenting with (multiple) combined LCNEC-adenocarcinoma. In addition, we aimed to identify clinically relevant subtypes of LCNEC patients who might benefit from adaptive treatment regimens. $A$ detailed investigation of clinical characteristics of LCNEC patients presenting with a solitary brain metastasis and patients with tumors with high proliferation rates but well differentiated morphology is provided in chapters $\mathbf{4}$ and $\mathbf{5}$.

Recently, the treatment of lung cancer has rapidly changed with the introduction of immunotherapy and treatment effect is correlated with a higher expression of programmed death-ligand 1 (Pd-I1) in tumor cells. Furthermore, recent studies have shown that high-grade neuroendocrine carcinomas generally express delta-like ligand 3 (DII3) which might be a target for therapy. ${ }^{24,25}$ In chapter $6-8$, we evaluated the 
expression of Pd-I1 and DII3 in LCNEC subtypes as possible predictive markers for treatment with immunotherapy and Dll3-targeted therapy. Finally, in chapter 9 we evaluated similarities and differences in metastatic patterns of neuroendocrine neoplasms (NEN) of various primary origins with a special focus on brain metastases. Herewith we explored the utility of screening for brain metastases in different NEN.

\section{Molecular subtypes}

\subsection{SCLC-like vs. NSCLC-like LCNEC}

In depth molecular evaluation utilizing next generation sequencing has led to the description of different molecular LCNEC subtypes. Generally, two main subtypes of LCNEC have been recognized. The first subtype has mutations in TP53 and STK11/KEAP1/KRAS (NSCLC-like), whereas the second subtype is co-mutated for TP53 and $R B 1$ (a hallmark of SCLC). ${ }^{10,11,26}$ This subclassification could also be made based on $\mathrm{pRb}$ immunohistochemistry (IHC) expression, classifying tumors with pRb loss as SCLClike. ${ }^{9}$ The latter results in a slightly different classification, since in addition to $R B 1$ mutations (or homozygous deletions/epigenetic inactivation) other mechanisms (e.g. p16 inactivation) can also result in pRb inactivation. ${ }^{27} \mathrm{~A}$ small subset of LCNEC with carcinoid-like features has also been identified, enriched for MEN1 mutations, but so far no criteria have been defined to recognize this subtype and current clinical value is unclear. ${ }^{11}$ In the vast majority of cases, the NSCLC- and SCLC-like subtypes are mutually exclusive. $^{10,11}$ Furthermore, differences in expression levels of ASCL1, DLL3 and neuroendocrine markers have been reported (high in NSCLC-like, low in SCLC-like). ${ }^{10}$ Two studies have evaluated the predictive value of SCLC- and NSCLC-like LCNEC on response to chemotherapy of SCLC or NSCLC regimens, however, with contrasting results. $^{9,17}$ Another limitation of the current classification is the overlap in observed gene mutations in some LCNEC cases. For example, co-mutation of KEAP1 with TP53 and RB1 occurs in a certain number of LCNEC and it is not clear if those cases should be regarded as SCLC-like or NSCLC-like. ${ }^{10,11}$ Furthermore, in this thesis, indications for molecular and clinical heterogeneity within both SCLC-like and NSCLC-like LCNEC were observed. First, in chapter 2, we showed that pulmonary oncologists and radiologists were unable to differentiate between SCLC-like and NSCLC-like LCNEC based on the interpretation of radiological images. Furthermore, neither semantic features of those images nor a radiomics signature could be used for this purpose. Moreover, most LCNEC were assessed as NSCLC-like by interpretation of the images and by the radiomics signature, whereas the majority of included LCNEC were of the molecular 
SCLC-like subtype, based on loss of $\mathrm{pRb}$ expression. This indicates that despite similar molecular characteristics, SCLC-like LCNEC has different radiological characteristics compared to SCLC. Second, in chapter 7, although we did not find a difference between DII3 expression in SCLC-like or NSCLC-like subtypes $(76 \%$ and $67 \%$ positivity, respectively), inhomogeneous results for DII3 staining were observed within the NSCLClike subtype. First, in a subgroup of samples with STK11 ( $\mathrm{N}=6)$ or KEAP1 $(\mathrm{N}=11)$ mutations, $100 \%$ and $91 \%$ of cases were positive for DIl3. A second subgroup within the NSCLC-like subtype contained samples with only one positive neuroendocrine marker and in this subgroup only $1 / 7(14 \%)$ samples was positive for Dll3. These inhomogeneous results might reflect the existence of additional LCNEC subtypes within NSCLC-like LCNEC.

\subsection{ASCL1, NEUROD1, POU2F3 and YAP1 subtypes}

The overlap between the SCLC-like and NSCLC-like LCNEC subtypes and the observed heterogeneity of the subtypes suggest that a different or additional subclassification could be more appropriate and clinically relevant. In that respect, a recent report on subclassification of SCLC is interesting to consider. ${ }^{28}$ In this report subclassification of SCLC in four groups has been proposed, based on gene expression levels of ASCL1 (70\%), NEUROD1 (11\%), POU2F3 (16\%) and YAP1 (2\%). ${ }^{28}$ The ASCL1 subtype represents the classic SCLC with TP53 and RB1 mutations and high expression of neuroendocrine markers. The remaining SCLC are represented by the POU2F3, YAP1 and NEUROD1 subtypes. In the latter three subtypes, RB1 mutations or neuroendocrine marker expression may be absent (especially in the POU2F3 subtype) and $\mathrm{pRb}$ expression might be retained. ${ }^{29}$ POU2F3, YAP1 and NEUROD1 are suggested to play a role in oncogenesis and neuroendocrine differentiation in those SCLC tumors. ${ }^{28}$ POU2F3 is a transcription factor, promoting the formation of a rare chemosensory cell type found in both respiratory and gastro-intestinal cells. Those cells are referred to as 'tuft cells' and upregulation of POU2F3 might induce tuft cell tumorigenesis. ${ }^{30}$ YAP1 is a downstream effector of the Hippo signaling pathway and promotes cell growth as a transcription cofactor. $^{31,32}$ NEUROD1 is involved in neuroendocrine differentiation and cell proliferation. $^{33,34}$ In a recent study using a mouse model inducing SCLC, it was observed that c-Myc (MYC) overexpression can activate NOTCH to dedifferentiate tumor cells thereby allowing temporal differentiation from SCLC ASCL1 subtype to SCLC NEUROD1 and YAP1 subtypes. ${ }^{35}$ Development of the SCLC POU2F3 subtype was also related to MYC activation in this study. ${ }^{35}$ Further research is necessary to confirm temporal differentiation and the relation with $M Y C$ activation, also in human tumors. It is tempting to speculate that ASCL1, NEUROD1, POU2F3 and YAP1 are also involved in 
oncogenesis of LCNEC. For example, NSCLC-like DII3 positive cases might be of the ASCL1 subtype, whereas NSCLC-like DII3 negative cases with expression of only 1 neuroendocrine marker might be of the YAP1 or POU2F3 subtype (Figure 10.1). Indeed, high mRNA expression levels of POU2F3 and YAP1 have already been shown in LCNEC with low ASCL1 expression. ${ }^{36}$

The subclassification in four tumor types might be relevant for treatment outcomes in SCLC and LCNEC, e.g. DII3-targeted therapy might be more efficient in the ASCL1 subtype whereas the YAP1 subtype might be less sensitive to cisplatin compared to general SCLC, but more sensitive to CDK4/6 inhibitors (Figure 10.1). ${ }^{28,31,37}$ However, further validation is necessary to determine clinical relevance.

Besides real SCLC subtypes, NEUROD1, YAP1 and POU2F3 subtypes could also represent cases on the borderline of LCNEC and SCLC, because inter-observer agreement to separate SCLC from LCNEC histopathologically is limited. ${ }^{38,39}$ Taking this into consideration, a classification of neuroendocrine carcinoma separated in molecular subtypes might be more reliable and more clinically relevant than the current separation between LCNEC and SCLC (Figure 10.1). Further studies on MRNA and protein expression levels of RB1, ASCL1, NEUROD1, POU2F3 and YAP1, in relation to histopathological and clinical characteristics, treatment outcome and survival should be performed in both SCLC and LCNEC to reveal clinical significance of those subtypes.

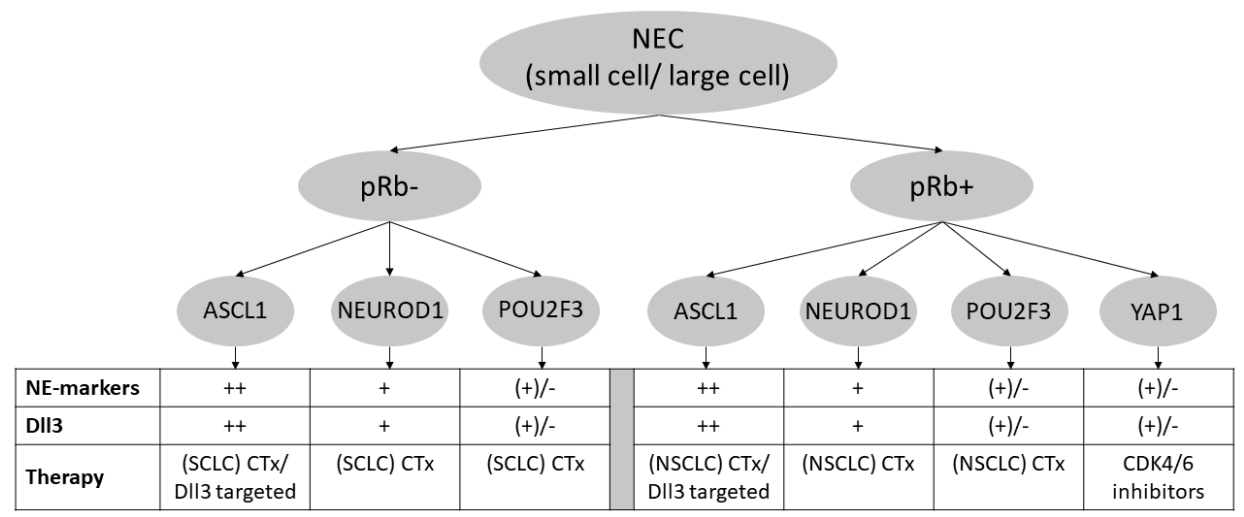

Figure 10.1 Proposed subclassification of both small cell and large cell neuroendocrine carcinoma, showing $\mathrm{pRb}+$ and $\mathrm{pRb}$ - subtypes with a subsequent partitioning in ASCL1, NEUROD1, POU2F3 and YAP1 subtypes. Hypothesized expression of neuroendocrine markers and DII3 and possible therapeutic options are provided for each subtype. Abbreviations: NEC = neuroendocrine carcinoma, $\mathrm{pRb}$ - = loss of immunohistochemical pRb expression (i.e. $\mathrm{H}$-score $<50$ ), $\mathrm{pRb}+=$ retained immunohistochemical pRb expression (i.e. $\mathrm{H}$-score $\geq 50$ ), $\mathrm{NE}$-markers = neuroendocrine markers, SCLC-like CTX = chemotherapeutic regimen according to small cell lung carcinoma guidelines (i.e. cisplatin + etoposide), NSCLC CTX = chemotherapeutic regimen according to nonsmall cell lung carcinoma guidelines (e.g. platinum combined with gemcitabine or paclitaxel). 


\subsection{New methods to reveal subtypes}

In the last decades, both IHC and mutational analysis have become widely available and analysis costs have dropped significantly. However, a considerable amount of pathological tissue is necessary if additional IHC has to be performed and even more when DNA/RNA has to be isolated for appropriate subclassification of the tumor. Especially in cases where only a (small) biopsy or cytology is available, this might be a challenge. An alternative could be to subclassify tumors by application of new technological developments in image analysis, for example radiomics. Radiomic signatures have been used previously to differentiate between SCLC and (subtypes of) NSCLC. ${ }^{40-43}$ Furthermore, primary origin of NET liver metastases can be predicted by semantic features. ${ }^{44}$ In chapter 2 we tried to identify SCLC-like and NSCLC-like LCNEC subtypes based on radiological images performed in routine diagnostic work-up. However, so far, we were unable to make a reliable classification based on interpretation of those images, semantic features and a radiomics signature.

Two other potential options for subclassification are the use of 'histomics' and 'proteomics'. With histomics, digitalized hematoxylin-eosin slides are analyzed for textural and statistical features, in analogy to radiomics methods. Digital pathology is evolving and extraction of quantitative features and deep learning algorithms have proven to be able to identify histologic or molecular subtypes of tumors. For example, two studies reported that SCLC and LCNEC could be diagnosed on cytology specimen by an algorithm, and molecular subtypes of bladder cancer could be determined based on the hematoxylin-eosin slide alone. ${ }^{45,46}$ However, development of histomics has only just started and LCNEC subtypes have not yet been investigated in this way.

In the last decade, proteomics has become an important field of oncology research. Protein analysis using shotgun sequencing and quantitative mass spectrometry can reveal an unique set of biomarkers for a (sub)type of cancer. Those biomarkers can be used for screening, diagnostic, prognostic and predictive purposes. ${ }^{47,48}$ So far, proteomics data on LCNEC is limited. In one study, Nomura et al. selected four representative proteins as biomarkers (Al1a1, Ak1c1, Ak1c3 and $C d 44)$, separating LCNEC from SCLC and large cell carcinoma. ${ }^{49}$ Al1a1 was confirmed in a subsequent investigation, which also revealed $4 \mathrm{f} 2 \mathrm{hc}$, Apoa1 and Enob as biomarkers for LCNEC. ${ }^{50}$ Another study revealed 1203 proteins shared by SCLC and LCNEC, and 195 proteins unique for SCLC and 254 only found in LCNEC. Despite these differences, clustering analysis could not separate SCLC and LCNEC tumors. ${ }^{51}$ So far, no investigations have been performed using proteomics to differentiate subtypes of LCNEC (i.e. SCLC-like vs. NSCLC-like), but a proteomic analysis of the secretome in ASCL1 and NEUROD1 SCLC subtypes revealed Igfbp5 as a marker for the ASCL1 high subtype. ${ }^{52}$ With the use of 
mass spectrometry, known proteins involved in development of LCNEC could be confirmed and new proteins might be discovered, improving the subdivision of LCNEC. However, for this method, fresh frozen material is preferred above formalin fixed paraffin embedded tissue and in general, fresh frozen material is not saved in routine clinical practice. Therefore, in retrospective research, only limited appropriate material will be available for a rare cancer type as LCNEC.

\section{Clinical subtypes}

\subsection{Combined LCNEC}

The heterogeneity of LCNEC is further illustrated by the subtype of combined LCNECadenocarcinoma (ADC) (chapter 3). ${ }^{53-55}$ The 10 presented cases all shared at least one mutation between LCNEC- and ADC-parts of the tumor, indicating a clonal relationship. $A D C$ related mutations (e.g. EGFR/KRAS/STK11 and KEAP1) were found in both tumor parts in 8/10 tumors, whereas those are usually detected in about half of pure LCNEC cases. $^{9-11,53-55}$ In addition, pRb inactivation was found in 6/10 LCNEC- and 4/10 ADCparts. This $\mathrm{pRb}$ inactivation is higher than expected in $A D C$, indicating an underlying role for $\mathrm{pRb}$ in the development of the combined tumors. ${ }^{56}$ The lower Ascl1 IHC expression in pure ADC, compared with ADC-parts of combined tumors and combined LCNEC-parts and pure LCNEC, suggested a role for Ascl1 in neuroendocrine development. An opposite pattern was observed for Rest1, with highest expression in pure ADC and combined ADC-parts. These interesting molecular characteristics contribute to the understanding of LCNEC oncogenesis. Unfortunately, we and others did not have the opportunity to investigate most effective treatment regimens in this subtype, due to low number of patients. ${ }^{53,55}$ It could be speculated that the choice of systemic treatment should be based on the LCNEC-part as this part might drive the prognosis, for example because of a higher $\mathrm{Ki}-67 \mathrm{PI}$ in those tumor parts compared to ADC-parts. Currently, a NSCLC regimen (including for example gemcitabine, docetaxel or paclitaxel) seems to be most appropriate in those combined tumors, as long as it is unclear which systemic treatment (SCLC regimen vs. NSCLC regimen) is optimal for LCNEC. $^{9,17}$ In addition to combined LCNEC-ADC tumors, LCNEC is also frequently combined with SCLC or squamous cell carcinoma. ${ }^{6,55,57,58}$ In-dept analysis of those two subtypes could also reveal additional information on LCNEC oncogenesis. 


\subsection{LCNEC with a solitary brain metastasis}

In chapters 4-5 two other subtypes with unique clinical and/or histopathological presentation were described. In chapter 4, 11 LCNEC patients with metastatic disease based on a solitary brain metastasis were identified. This is a unique subtype since metastatic LCNEC most often presents with extensive disseminated disease and only a few tumor series with oligometastatic disease have been described. ${ }^{1,3,59,60}$ Although no association was found with regard to NSCLC- or SCLC-like LCNEC subtypes, we did identify Ki-67 proliferation index (PI) as a possible prognostic factor. In patients with LCNEC having solitary brain metastases with a Ki-67 PI $\leq 40 \%$, the survival was better than expected for stage IV LCNEC (median overall survival 17 months). Therefore, patients presenting with a solitary brain metastasis with low proliferation might benefit from more aggressive treatment (e.g. metastasectomy) instead of palliative chemotherapy, in line with treatment of NSCLC patients with solitary brain metastases. $^{61}$ In general, Ki-67 PI $\leq 20 \%$ has been reported for carcinoids and Ki-67 PI $>40 \%$ for LCNEC. However, more metastatic and non-metastatic LCNEC with Ki-67 PI $\leq 40 \%$ might exist, which may be relevant for prognosis and therapy. ${ }^{62,63}$ For example, inferior response to platinum-based chemotherapeutic treatment has been shown for gastroenteral neuroendocrine carcinoma (NEC) with lower Ki-67 PI. ${ }^{64}$ Nonetheless, so far, the additional value of Ki-67 PI to the current WHO grading system of pulmonary NEN remains under debate. ${ }^{4,54,63,65,66}$ Further studies should therefore focus on LCNEC with a solitary brain metastasis and on LCNEC in general with Ki-67 PI $\leq 40 \%$ to reveal incidence, prognostic value and clinical relevance.

\subsection{LCNEC with well differentiated morphology}

Another clinical subtype was studied in chapter $\mathbf{5}$ and constituted of 7 patients with LCNEC with well differentiated morphology, but high proliferation rates (mitotic index (MI) $>10 / 2 \mathrm{~mm}^{2}$ and/or Ki-67 PI >20\%). In the current WHO classification (2015), this subtype is classified as LCNEC. ${ }^{4}$ However, in the WHO classification of gastroenteral pancreatic (GEP)-NEN, this subtype would be classified as a grade 3 neuroendocrine tumor (NET). ${ }^{67}$ With these 7 stage IV cases, we added to existing literature of small series mainly containing stage I-III patients. In general, a longer overall survival than expected for LCNEC was found in this subtype. ${ }^{12,15,68,69}$ We were the first to suggest pRb IHC staining as a prognostic marker in pulmonary NEN with well differentiated morphology and high proliferation rates (chapter 5). Nevertheless, a high frequency of disease recurrence has been described in this subtype and solid data on response to NEC focused treatments (e.g. platinum-etoposide) and NET focused treatments (e.g. everolimus or somatostatin analogues) is lacking. ${ }^{12,15,69-71}$ Therefore, future studies on 
stage I-IV NEN with well differentiated morphology and high proliferation rates should be performed to determine prognostic and therapeutic relevance.

\subsection{NSCLC transforming to LCNEC}

Another interesting subtype is LCNEC with an EGFR mutation, which has been transformed from NSCLC as a mechanism of resistance to EGFR-tyrosine kinase inhibitors (TKIs). ${ }^{56,72-82}$ Although this has extensively been described for SCLC, so far only a limited amount of cases with transformation to LCNEC has been reported. ${ }^{56,72-82}$ In those SCLC and LCNEC cases, a preserved EGFR mutation argues for a clonal relationship with the initial NSCLC and against development of a second primary tumor. The exact molecular changes underlying transformation remain unknown. $R B 1$ and TP53 have been described to be frequently mutated in those transformed tumors, whereas mutation rates of RB1 and TP53 in NSCLC with other resistance mechanisms to EGFR-TKIs are relatively low. ${ }^{56,72,74,75}$ Furthermore, NSCLC patients having inactivated pRb and p53 at baseline have $43 x$ greater risk of small-cell transformation. ${ }^{56}$ In addition, PIK3CA mutations have been shown to be present in transformed SCLC. ${ }^{74}$ Most likely, a mutational status predisposing for development of a neuroendocrine carcinoma (i.e. RB1 and TP53 mutations) is present at initial presentation of the EGFR mutated NSCLC cases, and during the course of EGFR-TKI, the tumors develop a neuroendocrine phenotype and herewith resistance to the TKI. Therefore, NSCLC patients with comutations of EGFR and RB1 and TP53 should be closely monitored, and re-biopsies should be taken in case of tumor progression during TKI treatment. Since only small series of transformed LCNEC has been described so far, incidence is unknown and this should be investigated in future nationwide studies.

\section{Markers for systemic treatment}

\section{$3.1 \quad$ Pd-I1}

In NSCLC, Pd-I1 expression ( $\geq 1 \%$ ) has been reported in up to $60 \%$ of tumors and $\mathrm{Pd}$ $1 / \mathrm{Pd}$-I1 targeted immunotherapy with or without chemotherapy is standard of care in stage IV patients without EGFR or ALK mutations. This has remarkedly increased overall survival in this group of patients. ${ }^{83-88}$ In contrast, approximately $10-30 \%$ of SCLC tumors are Pd-I1 positive. ${ }^{89-91}$ In chapter 8 , Pd-I1 expression was found in $16 \%$ of stage IV LCNEC, which is comparable to reported values of $9-32 \%$ in other studies including mainly early stage LCNEC. ${ }^{92-99}$ However, two recent studies seems to be contradictory with previous studies, reporting $\mathrm{Pd}-\mathrm{I} 1$ expression in $50 \%$ and $74 \%$ of $_{\text {cases. }}{ }^{100,101}$ 
Differences between both studies and the study on Pd-I1 expression in this thesis are the included population (mainly Asian vs. mainly Caucasian), tumor stage (mainly stage I-III vs. stage IV) and the Pd-I1 antibody clone used (E1L3N vs. 28-8). However, this does not explain the difference in expression between those two studies and other previous studies with better comparable methods, and it remains unclear why percentages are deviating. ${ }^{100,101}$ The relatively low percentage in the majority of studies resembles the percentages found in SCLC. This finding, in combination with the low percentage of Cd8 positive tumor infiltrating cells but a high number of $\mathrm{Cd} 8$ positive cells in the surrounding tissue (chapter 8), indicates that the majority of LCNEC is an 'immune excluded' tumor and only a low percentage is of the 'inflamed' tumor type. Therefore, combination therapy, for example with chemotherapy, might be more effective than monotherapy with immune checkpoint inhibition. For LCNEC, data on effectivity of immunotherapy is scarce and only small case series with responses lasting more than 6 months to Pd-I1 monotherapy as second and later-line treatment have been reported. $^{102-106}$ One case with complete response of a locally advanced LCNEC after palliative thoracic radiotherapy in combination with nivolumab has been described. ${ }^{107}$ For SCLC, the combination of chemotherapy with atezolizumab or durvalumab has shown a modest survival benefit, as has also been shown for the combination of nivolumab and ipilimumab. ${ }^{108-110}$ Furthermore, treatment of SCLC patients with pembrolizumab in combination with platinum-etoposide resulted in prolonged progression free survival compared to platinum-etoposide alone, although prolongation of overall survival was not significant. ${ }^{111}$ Those combinations might also be effective in LCNEC and this should be investigated in future clinical studies.

Despite the widely use of IHC staining with different antibody clones directed against $P d-I 1$, the predictive value of this marker for $P d-(I) 1$ therapy is limited, especially in tumors other than NSCLC and melanoma. ${ }^{112-114}$ Other markers and combinations of markers have been postulated in the past years, including $\mathrm{Cd} 8$ expression of tumor infiltrating cells, tumor mutational burden and imaging techniques. ${ }^{109,112,114-118}$ However, so far the value of all investigated markers is limited and future research should reveal more effective predictive markers to select patients, including LCNEC patients, for $\mathrm{Pd}-(\mathrm{I}) 1$ mono- or combination therapy.

\subsection{DII3}

DII3, one of the delta-like ligands in the DLL3-ASCL1-NOTCH1 pathway, involved in neuroendocrine differentiation, is expressed in the majority of SCLC and LCNEC tumors $(\approx 50-90 \%)$, but only very limited in healthy tissue (chapters 6-7). ${ }^{24,25,119-130}$ This makes DII3 a high potential therapeutic target in those tumors. However, so far clinically 
relevant cut-off values or the significance of a cytoplasmic/membranous or a punctuated staining pattern have not been clarified. Initial results in preclinical studies and a phase 1 study with the antibody-drug conjugate Rovalpituzumab-Tesirine (RovaT) were promising, but two subsequent phase 3 studies have been halted early due to low effectivity and high toxicity. ${ }^{24,25,86,131}$ A low dose of Rova-T in combination with Pd(I)1 therapy might have a clinical benefit, but so far, this has only been investigated in animal studies. ${ }^{132}$ Another antibody-drug conjugate was designed to overcome some of the toxicity problems of Rova-T (SC-002), but a phase I study showed serious systemic toxicity and only limited efficacy. No further development of this agent is planned. ${ }^{133}$ As an alternative, phase I studies with Bispecific T-cell Engaging $\left(\mathrm{BiTE}^{\circledR}\right)$ technique and Chimeric Antigen Receptor T-cell therapy targeting DII3 have been started, and results are awaited. ${ }^{134,135}$ Furthermore, a conjugate with Rovalpituzumab and the photosensitizer IR 700 has been developed for near infrared photoimmunotherapy, with promising results in preclinical studies. ${ }^{136}$ Results of ongoing and future studies have to be awaited to reveal if those high potential therapies for SCLC and LCNEC indeed will result in prolonged survival along with an acceptable toxicity profile.

\section{3 $\mathrm{pRb}$}

The identification of two main molecular subtypes of LCNEC, SCLC-like (RB1 mutation and/or loss of $\mathrm{pRb}$ expression) and NSCLC-like (RB1 wildtype and retained $\mathrm{pRb}$ expression), resulted in the hypothesis that treatment responses might be different in those subtypes. Indeed, for the NSCLC-like subtype, improved survival was observed in a Dutch cohort treated with NSCLC chemotherapeutic regimens (e.g. gemcitabine, paclitaxel, docetaxel) compared to patients treated with SCLC regimens (platinum + etoposide). This difference was not observed in the SCLC-like subtype. ${ }^{9}$ However, another study in mainly Asian patients found a trend for improved overall survival in the NSCLC-like subtype after treatment with platinum + etoposide compared to treatment with gemcitabine or taxanes. ${ }^{17} \mathrm{~A}$ possible explanation for this difference is the applied criterium for NSCLC-like LCNEC. Whilst the first study used RB1/TP53 mutations or loss of $\mathrm{pRb}$ expression, the latter only evaluated mutational loss of $R B 1$ and clustered all non-RB1 mutated LCNEC as NSCLC-like and thus did not account for potential other mechanisms of RB1 inactivation (i.e. homozygous deletions or epigenetic inactivation). Nevertheless, predictive relevance of the two subtypes requires prospective and when possible randomized evaluation in future studies. 


\subsection{Other markers}

Insulin-associated protein 1 (Insm1) has received attention as a neuroendocrine marker, which might outperform the traditional neuroendocrine markers regarding sensitivity and specificity, especially in SCLC (Insm1 sensitivity range: 86\%-100\%). ${ }^{137-142}$ Sensitivity in LCNEC has been reported to be somewhat lower (Insm1 sensitivity range: $42 \%-91 \%)$, but still seems to be higher than the sensitivity of chromogranin A with a comparable specificity (Chromogranin A sensitivity range: 33\%-48\%). ${ }^{137-142}$ Besides the use as a neuroendocrine marker, INSM1 has also been postulated as a therapeutic target in NEN. ${ }^{143}$ However, therapies are only in the first phases of development and animal studies or clinical trials have not been reported yet.

Other potential predictive markers are related to the proposed subclassification in ASCL1, NEUROD1, POU2F3 and YAP1 subtypes (see 1.2). For example, 3 Yap1-positive SCLC cell lines were significantly more resistant to cisplatin than 7 Yap1-negative SCLC cell lines and a trend for inferior chemotherapeutic response was also seen in Yap1 positive cell lines of another study. ${ }^{31,37}$ On the other hand, Yap1 positive cells are often pRb positive and seem to be more sensitive to CDK4/6 inhibitors than most SCLC (RB1 mutated). ${ }^{31}$ Further research should investigate those proposed markers in SCLC and LCNEC to reveal clinical relevance.

\section{Overlap with other NEN}

NEN of different primary origins have a number of similarities, for example histomorphological characteristics. However, differences also exist for instance between pulmonary and extrapulmonary NEN. These include that only $5 \%$ of pulmonary neuroendocrine tumors (NET) present with a functional syndrome, whereas in GEP-NET percentages up to $50 \%$ have been reported. ${ }^{144-146}$ Furthermore, the metastatic pattern of NEN from different primary origins is different, with more brain, bone and lung metastases in pulmonary NEN, as was shown in chapter 9. ${ }^{147}$ Another remarkable difference between GEP- and pulmonary NEN is the NET/NEC ratio. In GEPNEN, NET is more frequent with a ratio of $5-10: 1 .^{2}$ On the other hand, in pulmonary NEN, NEC is much more frequent ( $>7 x$, especially SCLC) compared to pulmonary carcinoids. ${ }^{2,144}$ This results in a relatively higher prevalence of GEP-NET compared to pulmonary NET and a much higher prevalence of pulmonary NEC compared to GEPNEC. ${ }^{148}$ As a consequence, most evidence of systemic treatment for NET is obtained from GEP-NET cohorts with less evidence available for pulmonary NET. Contrarily, SCLC regimens with platinum-etoposide are also administered as systemic treatment for NEC 
of extrapulmonary origin, but evidence from randomized controlled trials in nonpulmonary NEN is lacking. ${ }^{64,149-158}$ Although this transfer of knowledge on treatment possibilities from more prevalent neoplasms to less prevalent neoplasms is a logical step, caution should be exercised. In GEP-NEC and other extrapulmonary NEC, response rates to platinum-based chemotherapy are lower than in $\mathrm{SCLC}^{64,159,160}$ An explanation might be a different carcinogenesis, reflected by a weaker association with smoking as a risk factor and less TP53 and RB1 mutations in GEP-NEC (TP53 range 18\%-59\%, RB1 range $10 \%-34 \%$ ) compared to SCLC. ${ }^{161,162}$ Furthermore, more than half of GEP-NEC is of the large cell subtype. ${ }^{2,148}$ Even for pulmonary LCNEC, platinum-etoposide is doubted as the most optimal treatment strategy and NSCLC-like regimens might be more appropriate, especially in tumors with preserved pRb expression. ${ }^{9}$ Therefore, it is tempting to speculate that subtypes of GEP-NEC, e.g. large cell NEC with preserved pRb expression, might also benefit from other treatment regimens (e.g. fluorouracil-based chemotherapy).

So far, most research in the field of NEN focused on tumors from a specific primary origin, e.g. pancreatic or pulmonary NEN. In the future, maybe research could also be initiated with tumors from different primary origins but clear molecular and/or clinical similarities. For example, as stated above, treatment responses might have a stronger association with molecular subtypes (e.g. RB1 mutation) of NEN than with the primary origin itself. Future studies for systemic treatment could stratify for those molecular subtypes.

\section{WHO classification}

The WHO provides diagnostic criteria for cytology and histopathology for a broad range of tumors. This enables a classification and grading system, which is useful for prognostic purposes, treatment decisions and efficient communication between pathologists and the treating physician. However, sometimes it can be difficult to classify tumors according to the WHO system, as is illustrated by the cases described in chapter $\mathbf{5}$ and previous case series, with well differentiated morphology as a carcinoid characteristic and high proliferation rates more compatible with neuroendocrine carcinoma. ${ }^{4,11,12,14-16,68,163}$ The limited inter-observer agreement, for example between SCLC and LCNEC, further illustrates the restrictions of strict WHO classification. ${ }^{38,39}$ Moreover, in the past, differences in clinical characteristics, histopathology and molecular characteristics were regarded as evidence for a strict separation between carcinoids and high grade NEC (both SCLC and LCNEC). ${ }^{164,165}$ Nowadays, a temporal and spatial increase in proliferation rates in metastatic carcinoids, existence of a subgroup 
of carcinoids with molecular features of LCNEC (supra-carcinoids) and identification of a small LCNEC subgroup enriched for MEN1 mutations, question this strict separation. $^{11,163,166}$ Furthermore, a recent proof of concept study using two-way clustering of next generation sequencing data of carcinoids, SCLC and LCNEC, suggested development of secondary NEC from carcinoids. ${ }^{167}$ Besides this, in the current WHO classification all LCNEC are categorized together, whereas inter-tumor heterogeneity and the presence of clinical and molecular subtypes with possible clinical consequences has been suggested in this thesis and by others (chapters 2-5, 7). 4,9-16,68,163

These findings point out that the WHO classification cannot take all unique characteristics of each patient into consideration and in clinical practice, strict application of the WHO classification might result in suboptimal treatment in part of the LCNEC patients. Hence, it might be more suitable to regard LCNEC as part of the spectrum of pulmonary NEN, with unique subtypes but also overlapping features with NSCLC, SCLC and carcinoids (Figure 10.2).

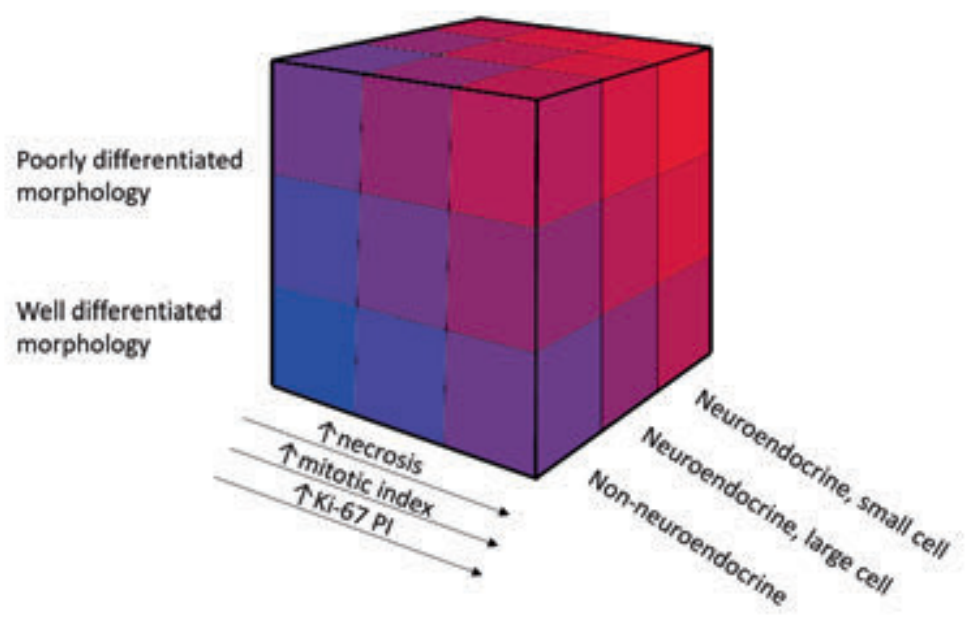

Figure 10.2 Hypothetical spectrum of large cell neuroendocrine carcinoma represented in three dimensions (morphology, cell type and proliferation). Blue color represents relatively indolent neoplasms, red color represents the most aggressive neoplasms. Abbreviations: Ki-67 PI $=\mathrm{Ki}-67$ proliferation index.

In clinical practice, a lot of information might be lost if the information transfer between the pathologist and the treating physician is mainly focused on the WHO diagnosis described in the final conclusion of the pathology report. This transfer could be improved if the pathologist provides the most important histopathological and 
molecular characteristics (e.g. pRb expression, Ki-67 PI) of the tumor to the clinician and gives an indication of the place in the neuroendocrine spectrum. By combining this pathological information with radiological and clinical information during a multidisciplinary team meeting, treatment plans can be further optimized.

For example, a stage IV patient with a tumor with poorly differentiated neuroendocrine morphology, $5 \%$ of the cells being small cell, $95 \%$ of the cells being non-small cell, abundant necrosis, MI 55/2 mm ${ }^{2}$, Ki-67 PI 70\% and loss of pRb expression, could be considered as having an aggressive neuroendocrine carcinoma, which should be treated with palliative platinum-etoposide. In this case, there seems to be no relevance for prognosis or treatment decisions to discriminate between SCLC or LCNEC. Another example is a patient who has undergone resection of a stage lb tumor. Pathology reveals a well differentiated neuroendocrine morphology, some necrosis, $\mathrm{MI} 12 / 2 \mathrm{~mm}^{2}$ and $\mathrm{Ki}-67 \mathrm{PI}$ 15\%. In case additional investigation reveals loss of $\mathrm{pRb}$ expression and negative staining for Orthopedia Homebox (Otp) and Cd44 (two potentially prognostic markers for carcinoids), the tumor is probably quite aggressive and close follow-up is advised. ${ }^{168}$ Alternatively, if pRb expression is preserved and/or Otp and Cd44 are positive, this tumor may be considered to be in the less aggressive part of the spectrum and recurrence of disease might be less likely, despite classification of this tumor as LCNEC by current WHO criteria. ${ }^{4}$

\section{Future perspectives}

In conclusion, LCNEC is a unique and heterogeneous disease harboring characteristics overlapping with SCLC, NSCLC and carcinoids. Molecular LCNEC subtypes likely exist, but SCLC-like and NSCLC-like subtypes were not reflected by radiological characteristics and an alternative molecular subclassification might be more appropriate. Furthermore, clinically relevant subtypes were identified, with possible implications for prognosis and treatment. DII3 targeted therapy might be beneficial for a subset of LCNEC, whereas the role of single agent Pd-(I)1 inhibition seems to be very limited. The current diagnostic criteria of the WHO classification should serve as the basis for communication between pathologists and clinicians. However, a more balanced way between application of those clearly defined diagnostic criteria and awareness of the spectrum of pulmonary NEN tumors and subtypes could be beneficial. Application of this spectrum and prognostic and predictive markers might further improve diagnosis and treatment for all unique pulmonary NEN patients and maximize outcome and quality of life. Future studies should aim to obtain additional insights in clinically 
relevant subtypes of $\mathrm{LCNEC}$, as well as in relevant predictive (e.g. molecular subtype, DII3 expression) and prognostic factors (e.g. Ki-67 PI). Furthermore, an accelerated increase of our insight of those rare tumor types could be obtained by transferring knowledge about one tumor type (e.g. GEP-NEN) to another tumor type (e.g. pulmonary NEN). Therefore, studies should also focus on molecular and/or clinical similarities and differences between NEN of different primary origins. In the future, artificial intelligence might be useful to include all pathological and clinical characteristics of NEN to aid clinicians in predicting prognosis and deciding on optimal treatment plans. 


\section{References}

1. Derks JL, Hendriks LE, Buikhuisen WA, et al. Clinical features of large cell neuroendocrine carcinoma: a population-based overview. Eur Respir J 2016;47:615-624.

2. Korse CM, Taal BG, van Velthuysen ML, et al. Incidence and survival of neuroendocrine tumours in the Netherlands according to histological grade: experience of two decades of cancer registry. Eur J Cancer 2013;49:1975-1983.

3. Takei $\mathrm{H}$, Asamura $\mathrm{H}$, Maeshima $\mathrm{A}$, et al. Large cell neuroendocrine carcinoma of the lung: a clinicopathologic study of eighty-seven cases. J Thorac Cardiovasc Surg 2002;124:285-292.

4. Travis WD, Brambilla E, Burke AP, et al. WHO Classification of Tumours of the Lung, Pleura, Thymus and Heart Lyon, France: International Agency for Research on Cancer; 2015.

5. Varlotto JM, Medford-Davis LN, Recht A, et al. Should large cell neuroendocrine lung carcinoma be classified and treated as a small cell lung cancer or with other large cell carcinomas? J Thorac Oncol 2011;6:1050-1058.

6. Asamura $\mathrm{H}$, Kameya $\mathrm{T}$, Matsuno $\mathrm{Y}$, et al. Neuroendocrine neoplasms of the lung: a prognostic spectrum. J Clin Oncol 2006;24:70-76.

7. Cao L, Li ZW, Wang M, et al. Clinicopathological characteristics, treatment and survival of pulmonary large cell neuroendocrine carcinoma: a SEER population-based study. PeerJ 2019;7:e6539.

8. Grondahl V, Binderup T, Langer SW, et al. Characteristics of 252 patients with bronchopulmonary neuroendocrine tumours treated at the Copenhagen NET Centre of Excellence. Lung Cancer 2019;132:141-149.

9. Derks JL, Leblay N, Thunnissen E, et al. Molecular Subtypes of Pulmonary Large-cell Neuroendocrine Carcinoma Predict Chemotherapy Treatment Outcome. Clin Cancer Res 2018;24:33-42.

10. George J, Walter V, Peifer M, et al. Integrative genomic profiling of large-cell neuroendocrine carcinomas reveals distinct subtypes of high-grade neuroendocrine lung tumors. Nat Commun 2018;9:1048.

11. Rekhtman N, Pietanza MC, Hellmann MD, et al. Next-Generation Sequencing of Pulmonary Large Cell Neuroendocrine Carcinoma Reveals Small Cell Carcinoma-like and Non-Small Cell Carcinoma-like Subsets. Clin Cancer Res 2016;22:3618-3629.

12. Quinn AM, Chaturvedi A, Nonaka D. High-grade Neuroendocrine Carcinoma of the Lung With Carcinoid Morphology: A Study of 12 Cases. Am J Surg Pathol 2017;41:263-270.

13. Rekhtman N, Desmeules $P$, Litvak $A M$, et al. Stage IV lung carcinoids: spectrum and evolution of proliferation rate, focusing on variants with elevated proliferation indices. Mod Pathol 2019;32:11061122.

14. Vivero M, Scholl LM. "Borderline" neuroendocrine carcinomas of the lung are clinically and genomically distinct from large cell neuroendocrine carcinoma. Mod Pathol 2016;29.

15. Kasajima A, Konukiewitz B, Oka N, et al. Clinicopathological Profiling of Lung Carcinoids with a Ki67 Index > 20. Neuroendocrinology 2019;108:109-120.

16. Oka N, Kasajima A, Konukiewitz B, et al. Classification and prognostic stratification of bronchopulmonary neuroendocrine neoplasms. Neuroendocrinology 2019.

17. Zhuo M, Guan Y, Yang X, et al. The prognostic and therapeutic role of genomic subtyping by sequencing tumor or cell-free DNA in pulmonary large-cell neuroendocrine carcinoma. Clin Cancer Res 2019.

18. Nanguzgambo AB, Aubeelack K, von Groote-Bidlingmaier F, et al. Radiologic features, staging, and operability of primary lung cancer in the Western cape, South Africa: a 1-year retrospective study. $J$ Thorac Oncol 2011;6:343-350.

19. Sahin F, Yildiz P. Radiological, Bronchoscopic and Histopathologic Characteristics of Patients with Primary Lung Cancer in Turkey (2006-2009). Asian Pac J Cancer Prev 2011;12:1947-1952.

20. Lee D, Rho JY, Kang S, et al. CT findings of small cell lung carcinoma: Can recognizable features be found? Medicine (Baltimore) 2016;95:e5426.

21. Hashimoto M, Heianna J, Okane K, et al. Small cell carcinoma of the lung: CT findings of parenchymal lesions. Radiat Med 1999;17:417-421. 
22. Kazawa N, Kitaichi M, Hiraoka M, et al. Small cell lung carcinoma: Eight types of extension and spread on computed tomography. J Comput Assist Tomogr 2006;30:653-661.

23. Ren $\mathrm{Y}, \mathrm{Cao} \mathrm{Y}, \mathrm{Hu} \mathrm{W}$, et al. Diagnostic accuracy of computed tomography imaging for the detection of differences between peripheral small cell lung cancer and peripheral non-small cell lung cancer. Int J Clin Oncol 2017;22:865-871.

24. Rudin CM, Pietanza MC, Bauer TM, et al. Rovalpituzumab tesirine, a DLL3-targeted antibody-drug conjugate, in recurrent small-cell lung cancer: a first-in-human, first-in-class, open-label, phase 1 study. Lancet Oncol 2017;18:42-51.

25. Saunders LR, Bankovich AJ, Anderson WC, et al. A DLL3-targeted antibody-drug conjugate eradicates high-grade pulmonary neuroendocrine tumor-initiating cells in vivo. Sci Transl Med 2015;7:302ra136.

26. Derks JL, Leblay N, Lantuejoul S, et al. New Insights into the Molecular Characteristics of Pulmonary Carcinoids and Large Cell Neuroendocrine Carcinomas, and the Impact on Their Clinical Management. J Thorac Oncol 2018;13:752-766.

27. Gouyer V, Gazzeri S, Bolon I, et al. Mechanism of retinoblastoma gene inactivation in the spectrum of neuroendocrine lung tumors. Am J Respir Cell Mol Biol 1998;18:188-196.

28. Rudin CM, Poirier JT, Byers LA, et al. Molecular subtypes of small cell lung cancer: a synthesis of human and mouse model data. Nat Rev Cancer 2019;19:289-297.

29. Baine MK, Hsieh MS, Lai WV, et al. Small Cell Lung Carcinoma Subtypes Defined by ASCL1, NEUROD1, POU2F3 and YAP1: Comprehensive Immunohistochemical and Histopathologic Characterization. J Thorac Oncol 2020.

30. Huang $\mathrm{YH}$, Klingbeil $\mathrm{O}, \mathrm{He} \mathrm{XY}$, et al. POU2F3 is a master regulator of a tuft cell-like variant of small cell lung cancer. Genes \& development 2018;32:915-928.

31. McColl K, Wildey G, Sakre N, et al. Reciprocal expression of INSM1 and YAP1 defines subgroups in small cell lung cancer. Oncotarget 2017;8:73745-73756.

32. Zhao B, Tumaneng K, Guan KL. The Hippo pathway in organ size control, tissue regeneration and stem cell self-renewal. Nature cell biology 2011;13:877-883.

33. Borromeo MD, Savage TK, Kollipara RK, et al. ASCL1 and NEUROD1 Reveal Heterogeneity in Pulmonary Neuroendocrine Tumors and Regulate Distinct Genetic Programs. Cell Rep 2016;16:1259-1272.

34. Hiroshima K, lyoda A, Shida T, et al. Distinction of pulmonary large cell neuroendocrine carcinoma from small cell lung carcinoma: a morphological, immunohistochemical, and molecular analysis. Mod Pathol 2006;19:1358-1368.

35. Ireland AS, Micinski AM, Kastner DW, et al. MYC Drives Temporal Evolution of Small Cell Lung Cancer Subtypes by Reprogramming Neuroendocrine Fate. Cancer Cell 2020.

36. Sonkin D, Thomas A, Teicher BA. Are neuroendocrine negative small cell lung cancer and large cell neuroendocrine carcinoma with WT RB1 two faces of the same entity? Lung cancer management 2019;8:Lmt13.

37. Ito T, Matsubara D, Tanaka I, et al. Loss of YAP1 defines neuroendocrine differentiation of lung tumors. Cancer Sci 2016;107:1527-1538.

38. Ha SY, Han J, Kim WS, et al. Interobserver variability in diagnosing high-grade neuroendocrine carcinoma of the lung and comparing it with the morphometric analysis. Korean journal of pathology 2012;46:42-47.

39. den Bakker MA, Willemsen S, Grunberg K, et al. Small cell carcinoma of the lung and large cell neuroendocrine carcinoma interobserver variability. Histopathology 2010;56:356-363.

40. Zhang J, Jin J, Ai Y, et al. Differentiating the pathological subtypes of primary lung cancer for patients with brain metastases based on radiomics features from brain CT images. European radiology 2020.

41. E L, Lu L, Li L, et al. Radiomics for Classification of Lung Cancer Histological Subtypes Based on Nonenhanced Computed Tomography. Acad Radiol 2018.

42. E L, Lu L, Li L, et al. Radiomics for Classifying Histological Subtypes of Lung Cancer Based on Multiphasic Contrast-Enhanced Computed Tomography. J Comput Assist Tomogr 2019;43:300-306.

43. Ren C, Zhang J, Qi M, et al. Machine learning based on clinico-biological features integrated (18)F-FDG $\mathrm{PET} / \mathrm{CT}$ radiomics for distinguishing squamous cell carcinoma from adenocarcinoma of lung. European journal of nuclear medicine and molecular imaging 2020. 
44. Gulpinar B, Peker E, Kul M, et al. Liver metastases of neuroendocrine tumors: is it possible to diagnose different histologic subtypes depending on multiphasic CT features? Abdominal radiology (New York) 2019;44:2147-2155.

45. Woerl AC, Eckstein M, Geiger J, et al. Deep Learning Predicts Molecular Subtype of Muscle-invasive Bladder Cancer from Conventional Histopathological Slides. European urology 2020.

46. Gonzalez D, Dietz RL, Pantanowitz L. Feasibility of a Deep Learning Algorithm to Distinguish Large Cell Neuroendocrine from Small Cell Lung Carcinoma in Cytology Specimens. Cytopathology : official journal of the British Society for Clinical Cytology 2020.

47. Belczacka I, Latosinska A, Metzger J, et al. Proteomics biomarkers for solid tumors: Current status and future prospects. Mass Spectrom Rev 2018.

48. Jiang F, Zhou XY, Huang J. The value of surface enhanced laser desorption/ionization-time of flight mass spectrometry at the diagnosis of non-small cell lung cancer: a systematic review. Technol Cancer Res Treat 2014;13:109-117.

49. Nomura M, Fukuda T, Fujii K, et al. Preferential expression of potential markers for cancer stem cells in large cell neuroendocrine carcinoma of the lung. An FFPE proteomic study. J Clin Bioinforma 2011;1:23.

50. Fukuda $\mathrm{T}$, Nomura $\mathrm{M}$, Kato $\mathrm{Y}$, et al. A selected reaction monitoring mass spectrometric assessment of biomarker candidates diagnosing large-cell neuroendocrine lung carcinoma by the scaling method using endogenous references. PloS one 2017;12:e0176219.

51. Nakamura H, Fujii K, Gupta V, et al. Identification of key modules and hub genes for small-cell lung carcinoma and large-cell neuroendocrine lung carcinoma by weighted gene co-expression network analysis of clinical tissue-proteomes. PloS one 2019;14:e0217105.

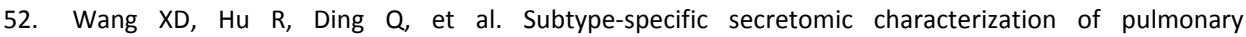
neuroendocrine tumor cells. Nat Commun 2019;10:3201.

53. Ito M, Miyata $\mathrm{Y}$, Hirano $\mathrm{S}$, et al. Therapeutic strategies and genetic profile comparisons in small cell carcinoma and large cell neuroendocrine carcinoma of the lung using next-generation sequencing. Oncotarget 2017;8:108936-108945.

54. Milione M, Maisonneuve P, Grillo F, et al. Ki-67 index of 55\% distinguishes two groups of bronchopulmonary pure and composite large cell neuroendocrine carcinomas with distinct prognosis. Neuroendocrinology 2020.

55. Miyoshi T, Umemura S, Matsumura Y, et al. Genomic Profiling of Large-Cell Neuroendocrine Carcinoma of the Lung. Clin Cancer Res 2017;23:757-765.

56. Lee JK, Lee J, Kim S, et al. Clonal History and Genetic Predictors of Transformation Into Small-Cell Carcinomas From Lung Adenocarcinomas. J Clin Oncol 2017;35:3065-3074.

57. Zhang JT, Li Y, Yan LX, et al. Disparity in clinical outcomes between pure and combined pulmonary large-cell neuroendocrine carcinoma: A multi-center retrospective study. Lung Cancer 2020;139:118123.

58. Zhang $\mathrm{C}$, Yang $\mathrm{H}$, Zhao $\mathrm{H}$, et al. Clinical outcomes of surgically resected combined small cell lung cancer: a two-institutional experience. J Thorac Dis 2017;9:151-158.

59. Kotecha R, Zimmerman A, Murphy ES, et al. Management of Brain Metastasis in Patients With Pulmonary Neuroendocrine Carcinomas. Technol Cancer Res Treat 2016;15:566-572.

60. Naidoo J, Santos-Zabala ML, Iyriboz T, et al. Large Cell Neuroendocrine Carcinoma of the Lung: ClinicoPathologic Features, Treatment, and Outcomes. Clin Lung Cancer 2016;17:e121-e129.

61. Li D, Zhu X, Wang $\mathrm{H}$, et al. Should aggressive thoracic therapy be performed in patients with synchronous oligometastatic non-small cell lung cancer? A meta-analysis. J Thorac Dis 2017;9:310-317.

62. Pelosi G, Rindi G, Travis WD, et al. Ki-67 antigen in lung neuroendocrine tumors: unraveling a role in clinical practice. J Thorac Oncol 2014;9:273-284.

63. Fabbri A, Cossa M, Sonzogni A, et al. Ki-67 labeling index of neuroendocrine tumors of the lung has a high level of correspondence between biopsy samples and surgical specimens when strict counting guidelines are applied. Virchows Arch 2017;470:153-164.

64. Sorbye H, Welin S, Langer SW, et al. Predictive and prognostic factors for treatment and survival in 305 patients with advanced gastrointestinal neuroendocrine carcinoma (WHO G3): the NORDIC NEC study. Ann Oncol 2013;24:152-160. 
65. Rindi G, Klersy C, Inzani F, et al. Grading the neuroendocrine tumors of the lung: an evidence-based proposal. Endocr Relat Cancer 2014;21:1-16.

66. Walts AE, Mirocha JM, Marchevsky AM. Challenges in Ki-67 assessments in pulmonary large cell neuroendocrine carcinomas. Histopathology 2020.

67. WHO. WHO classification of Tumours, Digestive System Tumours, 5th edition. WHO; 2019.

68. Inafuku $\mathrm{K}$, Yokose $\mathrm{T}$, Ito $\mathrm{H}$, et al. Two cases of lung neuroendocrine carcinoma with carcinoid morphology. Diagn Pathol 2019;14:104.

69. Rubino M, Scoazec JY, Pisa E, et al. Lung carcinoids with high proliferative activity: Further support for the identification of a new tumor category in the classification of lung neuroendocrine neoplasms. Lung Cancer 2020;148:149-158.

70. Cros J, Theou-Anton N, Gounant V, et al. Specific genomic alterations in high grade pulmonary neuroendocrine tumours with carcinoid morphology. Neuroendocrinology 2020.

71. Sazonova O, Manem V, Orain M, et al. Transcriptomic data helps refining classification of pulmonary carcinoid tumors with increased mitotic counts. Mod Pathol 2020.

72. Niederst MJ, Sequist LV, Poirier JT, et al. RB loss in resistant EGFR mutant lung adenocarcinomas that transform to small-cell lung cancer. Nat Commun 2015;6:6377.

73. Yu HA, Arcila ME, Rekhtman N, et al. Analysis of tumor specimens at the time of acquired resistance to EGFR-TKI therapy in 155 patients with EGFR-mutant lung cancers. Clin Cancer Res 2013;19:2240-2247.

74. Marcoux N, Gettinger SN, O'Kane G, et al. EGFR-Mutant Adenocarcinomas That Transform to Small-Cell Lung Cancer and Other Neuroendocrine Carcinomas: Clinical Outcomes. J Clin Oncol 2019;37:278-285.

75. Aggarwal C, Davis CW, Mick R, et al. Influence of TP53 Mutation on Survival in Patients With Advanced EGFR-Mutant Non-Small-Cell Lung Cancer. JCO Precis Oncol 2018;2018.

76. Ferrer L, Giaj Levra M, Brevet M, et al. A Brief Report of Transformation From NSCLC to SCLC: Molecular and Therapeutic Characteristics. J Thorac Oncol 2019;14:130-134.

77. Yanagisawa S, Morikawa N, Kimura Y, et al. Large-cell neuroendocrine carcinoma with epidermal growth factor receptor mutation: possible transformation of lung adenocarcinoma. Respirology 2012;17:1275-1277.

78. Baglivo S, Ludovini V, Sidoni A, et al. Large Cell Neuroendocrine Carcinoma Transformation and EGFRT790M Mutation as Coexisting Mechanisms of Acquired Resistance to EGFR-TKIs in Lung Cancer. Mayo Clinic proceedings 2017;92:1304-1311.

79. Lim JU, Woo IS, Jung YH, et al. Transformation into large-cell neuroendocrine carcinoma associated with acquired resistance to erlotinib in nonsmall cell lung cancer. Korean J Intern Med 2014;29:830-833.

80. Kogo M, Shimizu R, Uehara K, et al. Transformation to large cell neuroendocrine carcinoma as acquired resistance mechanism of EGFR tyrosine kinase inhibitor. Lung Cancer 2015;90:364-368.

81. Moriguchi S, Uruga H, Fujii T, et al. Transformation of epidermal growth factor receptor T790M mutation-positive adenosquamous carcinoma of the lung to small cell carcinoma and large-cell neuroendocrine carcinoma following osimertinib therapy: an autopsy case report. Respirol Case Rep 2019;7:e00402.

82. Moriya R, Hokari S, Shibata S, et al. Histological Transformation to Large Cell Neuroendocrine Carcinoma from Lung Adenocarcinoma Harboring an EGFR Mutation: An Autopsy Case Report. Intern Med 2017;56:2013-2017.

83. Novello S, Barlesi F, Califano R, et al. Metastatic non-small-cell lung cancer: ESMO Clinical Practice Guidelines for diagnosis, treatment and follow-up. Ann Oncol 2016;27:v1-v27.

84. Hanna N, Johnson D, Temin S, et al. Systemic Therapy for Stage IV Non-Small-Cell Lung Cancer: American Society of Clinical Oncology Clinical Practice Guideline Update. J Clin Oncol 2017;35:34843515.

85. Mu CY, Huang JA, Chen Y, et al. High expression of PD-L1 in lung cancer may contribute to poor prognosis and tumor cells immune escape through suppressing tumor infiltrating dendritic cells maturation. Med Oncol 2011;28:682-688.

86. Chan AWH, Tong JHM, Kwan JSH, et al. Assessment of programmed cell death ligand-1 expression by 4 diagnostic assays and its clinicopathological correlation in a large cohort of surgical resected non-small cell lung carcinoma. Mod Pathol 2018 [Epub ahead of print]. 
87. Cooper WA, Tran T, Vilain RE, et al. PD-L1 expression is a favorable prognostic factor in early stage nonsmall cell carcinoma. Lung Cancer 2015;89:181-188.

88. Gandhi L, Rodriguez-Abreu D, Gadgeel S, et al. Pembrolizumab plus Chemotherapy in Metastatic NonSmall-Cell Lung Cancer. N Engl J Med 2018;378:2078-2092.

89. Antonia SJ, Lopez-Martin JA, Bendell J, et al. Nivolumab alone and nivolumab plus ipilimumab in recurrent small-cell lung cancer (CheckMate 032): a multicentre, open-label, phase 1/2 trial. Lancet Oncol 2016;17:883-895.

90. Ott PA, Elez E, Hiret S, et al. Pembrolizumab in Patients With Extensive-Stage Small-Cell Lung Cancer: Results From the Phase Ib KEYNOTE-028 Study. J Clin Oncol 2017;35:3823-3829.

91. Gadgeel SM, Pennell NA, Fidler MJ, et al. Phase II Study of Maintenance Pembrolizumab in Patients with Extensive-Stage Small Cell Lung Cancer (SCLC). J Thorac Oncol 2018;13:1393-1399.

92. Kasajima A, Ishikawa $Y$, Iwata A, et al. Inflammation and PD-L1 expression in pulmonary neuroendocrine tumors. Endocr Relat Cancer 2018;25:339-350.

93. Tsuruoka K, Horinouchi H, Goto Y, et al. PD-L1 expression in neuroendocrine tumors of the lung. Lung Cancer 2017;108:115-120.

94. Kim HS, Lee JH, Nam SJ, et al. Association of PD-L1 Expression with Tumor-Infiltrating Immune Cells and Mutation Burden in High-Grade Neuroendocrine Carcinoma of the Lung. J Thorac Oncol 2018;13:636648.

95. Eichhorn F, Harms A, Warth A, et al. PD-L1 expression in large cell neuroendocrine carcinoma of the lung. Lung Cancer 2018;118:76-82.

96. Takada K, Toyokawa G, Okamoto T, et al. Metabolic characteristics of programmed cell death-ligand 1expressing lung cancer on (18) F-fluorodeoxyglucose positron emission tomography/computed tomography. Cancer Med 2017;6:2552-2561.

97. Inamura K, Yokouchi Y, Kobayashi M, et al. Relationship of tumor PD-L1 (CD274) expression with lower mortality in lung high-grade neuroendocrine tumor. Cancer Med 2017;6:2347-2356.

98. Wang H, Li Z, Dong B, et al. Prognostic significance of PD-L1 expression and CD8+ T cell infiltration in pulmonary neuroendocrine tumors. Diagn Pathol 2018;13:30.

99. Arpin D, Charpentier MC, Bernardi M, et al. PD-L1-expression patterns in large-cell neuroendocrine carcinoma of the lung: potential implications for use of immunotherapy in these patients: the GFPC 032017 "EPNEC" study. Therapeutic advances in medical oncology 2020;12:1758835920937972.

100. Ohtaki Y, Kaira K, Atsumi J, et al. Prognostic significance of PD-L1 expression and tumor infiltrating lymphocytes in large cell neuroendocrine carcinoma of lung. American journal of translational research 2018;10:3243-3253.

101. Ichiki Y, Matsumiya $\mathrm{H}$, Mori $\mathrm{M}$, et al. Predictive factors of postoperative survival among patients with pulmonary neuroendocrine tumor. J Thorac Dis 2018;10:6912-6920.

102. Levra MG, Mazieres J, C.A. V, et al. Efficacy of immune checkpoint inhibitors in large cell neuroendocrine lung cancer Journal of Thoracic Oncology 2017;12:S702-S703.

103. Wang VE, Urisman A, Albacker L, et al. Checkpoint inhibitor is active against large cell neuroendocrine carcinoma with high tumor mutation burden. J Immunother Cancer 2017;5:75.

104. Daido W, Yamasaki M, Saito N, et al. [Effectiveness of Nivolumab in Large-Cell Neuroendocrine Carcinoma of the Lung - A Report of Two Cases]. Gan To Kagaku Ryoho 2017;44:59-62.

105. Sherman S, Rotem O, Shochat T, et al. Efficacy of immune check-point inhibitors (ICPi) in large cell neuroendocrine tumors of lung (LCNEC). Lung Cancer 2020;143:40-46.

106. Zhang X, Sun Y, Miao Y, et al. Immune Checkpoint Inhibitor Therapy Achieved Complete Response for Drug-Sensitive EGFR/ALK Mutation-Negative Metastatic Pulmonary Large-Cell Neuroendocrine Carcinoma with High Tumor Mutation Burden: A Case Report. Onco Targets Ther 2020;13:8245-8250.

107. Mauclet C, Duplaquet F, Pirard L, et al. Complete tumor response of a locally advanced lung large-cell neuroendocrine carcinoma after palliative thoracic radiotherapy and immunotherapy with nivolumab. Lung Cancer 2019;128:53-56.

108. Horn L, Mansfield AS, Szczesna A, et al. First-Line Atezolizumab plus Chemotherapy in Extensive-Stage Small-Cell Lung Cancer. N Engl J Med 2018;379:2220-2229. 
109. Hellmann MD, Callahan MK, Awad MM, et al. Tumor Mutational Burden and Efficacy of Nivolumab Monotherapy and in Combination with Ipilimumab in Small-Cell Lung Cancer. Cancer Cell 2018;33:853861 e854.

110. Paz-Ares L, Dvorkin M, Chen $\mathrm{Y}$, et al. Durvalumab plus platinum-etoposide versus platinum-etoposide in first-line treatment of extensive-stage small-cell lung cancer (CASPIAN): a randomised, controlled, open-label, phase 3 trial. Lancet 2019;394:1929-1939.

111. Rudin CM, Awad MM, Navarro A, et al. Pembrolizumab or Placebo Plus Etoposide and Platinum as FirstLine Therapy for Extensive-Stage Small-Cell Lung Cancer: Randomized, Double-Blind, Phase III KEYNOTE604 Study. J Clin Oncol 2020:Jco2000793.

112. Niemeijer AN, Sahba S, Smit EF, et al. Association of tumour and stroma PD-1, PD-L1, CD3, CD4 and CD8 expression with DCB and OS to nivolumab treatment in NSCLC patients pre-treated with chemotherapy. British journal of cancer 2020.

113. Garon EB, Rizvi NA, Hui R, et al. Pembrolizumab for the treatment of non-small-cell lung cancer. $N$ Engl J Med 2015;372:2018-2028.

114. Krieger T, Pearson I, Bell J, et al. Targeted literature review on use of tumor mutational burden status and programmed cell death ligand 1 expression to predict outcomes of checkpoint inhibitor treatment. Diagn Pathol 2020;15:6.

115. Rizvi H, Sanchez-Vega F, La K, et al. Molecular Determinants of Response to Anti-Programmed Cell Death (PD)-1 and Anti-Programmed Death-Ligand 1 (PD-L1) Blockade in Patients With Non-Small-Cell Lung Cancer Profiled With Targeted Next-Generation Sequencing. J Clin Oncol 2018;36:633-641.

116. Snyder A, Makarov V, Merghoub T, et al. Genetic basis for clinical response to CTLA-4 blockade in melanoma. N Engl J Med 2014;371:2189-2199.

117. Bensch F, van der Veen EL, Lub-de Hooge MN, et al. (89)Zr-atezolizumab imaging as a non-invasive approach to assess clinical response to PD-L1 blockade in cancer. Nature medicine 2018;24:1852-1858.

118. Marabelle A, Fakih M, Lopez J, et al. Association of tumour mutational burden with outcomes in patients with advanced solid tumours treated with pembrolizumab: prospective biomarker analysis of the multicohort, open-label, phase 2 KEYNOTE-158 study. Lancet Oncol 2020;21:1353-1365.

119. Saito $M$, Saito K, Shiraishi K, et al. Identification of candidate responders for anti-PD-L1/PD-1 immunotherapy, Rova-T therapy, or EZH2 inhibitory therapy in small-cell lung cancer. Mol Clin Oncol 2018;8:310-314.

120. Tanaka K, Isse K, Fujihira T, et al. Prevalence of Delta-like protein 3 expression in patients with small cell lung cancer. Lung Cancer 2018;115:116-120.

121. Brcic L, Kuchler C, Eidenhammer S, et al. Comparison of four DLL3 antibodies performance in high grade neuroendocrine lung tumor samples and cell cultures. Diagn Pathol 2019;14:47.

122. Huang RSP, Holmes BF, Powell C, et al. Delta-like Protein 3 Prevalence in Small Cell Lung Cancer and DLL3 (SP347) Assay Characteristics. Arch Pathol Lab Med 2019.

123. Furuta M, Sakakibara-Konishi J, Kikuchi H, et al. Analysis of DLL3 and ASCL1 in Surgically Resected Small Cell Lung Cancer (HOT1702). Oncologist 2019;24:e1172-e1179.

124. Roy S, Martinez K, Dilip A, et al. DLL3 analysis of circulating tumor cells predict treatment in phase 1 rova-T study in small cell lung cancer (Abstract \#3721). AACR. Washington, DC: 2017;Abstracts 30635947.

125. Xie H, Boland JM, Maleszewski JJ, et al. Expression of delta-like protein 3 is reproducibly present in a subset of small cell lung carcinomas and pulmonary carcinoid tumors. Lung Cancer 2019;135:73-79.

126. Messaritakis I, Nikolaou M, Koinis F, et al. Characterization of DLL3-positive circulating tumor cells (CTCS) in patients with small cell lung cancer (SCLC) and evaluation of their clinical relevance during front-line treatment. Lung Cancer 2019;135:33-39.

127. Regzedmaa O, Li Y, Li Y, et al. Prevalence of DLL3, CTLA-4 and MSTN Expression in Patients with Small Cell Lung Cancer. Onco Targets Ther 2019;12:10043-10055.

128. Yan LX, Liu YH, Li Z, et al. Prognostic value of delta-like protein 3 combined with thyroid transcription factor-1 in small-cell lung cancer. Oncol Lett 2019;18:2254-2261.

129. Ogawa H, Sakai Y, Nishio W, et al. DLL3 expression is a predictive marker of sensitivity to adjuvant chemotherapy for pulmonary LCNEC. Thoracic cancer 2020;11:2561-2569. 
130. Rojo F, Corassa M, Mavroudis D, et al. International real-world study of DLL3 expression in patients with small cell lung cancer. Lung Cancer 2020;147:237-243.

131. AbbVie Discontinues Rovalpituzumab Tesirine (Rova-T) Research and Development Program [press release]. Abbvie, North Chicago; 2019.

132. Vitorino $\mathrm{P}$, Chuang $\mathrm{CH}$, lannello $\mathrm{A}$, et al. Rova-T enhances the anti-tumor activity of anti-PD1 in a murine model of small cell lung cancer with endogenous Dll3 expression. Translational oncology 2020;14:100883.

133. Morgensztern D, Johnson M, Rudin CM, et al. SC-002 in patients with relapsed or refractory small cell lung cancer and large cell neuroendocrine carcinoma: Phase 1 study. Lung Cancer 2020;145:126-131.

134. Giffin M, Cooke K, Lobenhofer E, et al. Targeting DLL3 with AMG 757, a BiTE ${ }^{\circledR}$ Antibody Construct, and AMG 119, a CAR-T, for the Treatment of SCLC. J Thorac Oncol 2018;13:Supplement.

135. Hipp S, Voynov V, Drobits-Handl B, et al. A novel T-cell engaging bispecific antibody induces specific and efficacious lysis of small cell lung cancer cells in vitro and potent $T$ cell re-directed anti-tumor activity in vivo (Abstract \#549). AACR. Atlanta: 2019;Part A: Abstracts 1-2748.

136. Isobe $Y$, Sato K, Nishinaga $Y$, et al. Near infrared photoimmunotherapy targeting DLL3 for small cell lung cancer. EBioMedicine 2020;52:102632.

137. Kriegsmann K, Zgorzelski C, Kazdal D, et al. Insulinoma-associated Protein 1 (INSM1) in Thoracic Tumors is Less Sensitive but More Specific Compared With Synaptophysin, Chromogranin A, and CD56. Applied immunohistochemistry \& molecular morphology : AIMM 2020;28:237-242.

138. Viswanathan K, Siddiqui MT, Borczuk AC. Insulinoma-associated protein 1 is a sensitive and specific marker for lung neuroendocrine tumors in cytologic and surgical specimens. Journal of the American Society of Cytopathology 2019;8:299-308.

139. Mukhopadhyay S, Dermawan JK, Lanigan CP, et al. Insulinoma-associated protein 1 (INSM1) is a sensitive and highly specific marker of neuroendocrine differentiation in primary lung neoplasms: an immunohistochemical study of 345 cases, including 292 whole-tissue sections. Mod Pathol 2019;32:100-109.

140. Sakakibara R, Kobayashi M, Takahashi N, et al. Insulinoma-associated Protein 1 (INSM1) Is a Better Marker for the Diagnosis and Prognosis Estimation of Small Cell Lung Carcinoma Than Neuroendocrine Phenotype Markers Such as Chromogranin A, Synaptophysin, and CD56. Am J Surg Pathol 2020;44:757764.

141. Rooper LM, Sharma R, Li QK, et al. INSM1 Demonstrates Superior Performance to the Individual and Combined Use of Synaptophysin, Chromogranin and CD56 for Diagnosing Neuroendocrine Tumors of the Thoracic Cavity. Am J Surg Pathol 2017;41:1561-1569.

142. Minami K, Jimbo N, Tanaka $\mathrm{Y}$, et al. Insulinoma-associated protein 1 is a prognostic biomarker in pulmonary high-grade neuroendocrine carcinoma. Journal of surgical oncology 2020.

143. Mahalakshmi B, Baskaran R, Shanmugavadivu M, et al. Insulinoma-associated protein 1 (INSM1): a potential biomarker and therapeutic target for neuroendocrine tumors. Cellular oncology (Dordrecht) 2020;43:367-376.

144. Gustafsson BI, Kidd M, Chan A, et al. Bronchopulmonary neuroendocrine tumors. Cancer 2008;113:521.

145. Jann H, Roll S, Couvelard A, et al. Neuroendocrine tumors of midgut and hindgut origin: tumor-nodemetastasis classification determines clinical outcome. Cancer 2011;117:3332-3341.

146. Pape UF, Jann H, Müller-Nordhorn J, et al. Prognostic relevance of a novel TNM classification system for upper gastroenteropancreatic neuroendocrine tumors. Cancer 2008;113:256-265.

147. Riihimaki M, Hemminki A, Sundquist $K$, et al. The epidemiology of metastases in neuroendocrine tumors. Int J Cancer 2016;139:2679-2686.

148. Dasari A, Mehta K, Byers LA, et al. Comparative study of lung and extrapulmonary poorly differentiated neuroendocrine carcinomas: A SEER database analysis of 162,983 cases. Cancer 2018;124:807-815.

149. Lamarca A, Frizziero M, Barriuso J, et al. Urgent need for consensus: international survey of clinical practice exploring use of platinum-etoposide chemotherapy for advanced extra-pulmonary high grade neuroendocrine carcinoma (EP-G3-NEC). Clin Transl Oncol 2019;21:950-953.

150. Heetfeld $\mathrm{M}$, Chougnet $\mathrm{CN}$, Olsen $\mathrm{IH}$, et al. Characteristics and treatment of patients with $\mathrm{G} 3$ gastroenteropancreatic neuroendocrine neoplasms. Endocr Relat Cancer 2015;22:657-664. 
151. Moertel CG, Kvols LK, O'Connell MJ, et al. Treatment of neuroendocrine carcinomas with combined etoposide and cisplatin. Evidence of major therapeutic activity in the anaplastic variants of these neoplasms. Cancer 1991;68:227-232.

152. Pavel M, O'Toole D, Costa F, et al. ENETS Consensus Guidelines Update for the Management of Distant Metastatic Disease of Intestinal, Pancreatic, Bronchial Neuroendocrine Neoplasms (NEN) and NEN of Unknown Primary Site. Neuroendocrinology 2016;103:172-185.

153. Pavel M, Öberg K, Falconi M, et al. Gastroenteropancreatic neuroendocrine neoplasms: ESMO Clinical Practice Guidelines for diagnosis, treatment and follow-up. Ann Oncol 2020;31:844-860.

154. Fjällskog ML, Granberg DP, Welin SL, et al. Treatment with cisplatin and etoposide in patients with neuroendocrine tumors. Cancer 2001;92:1101-1107.

155. Mitry E, Baudin E, Ducreux M, et al. Treatment of poorly differentiated neuroendocrine tumours with etoposide and cisplatin. British journal of cancer 1999;81:1351-1355.

156. Ilett EE, Langer SW, Olsen IH, et al. Neuroendocrine Carcinomas of the Gastroenteropancreatic System: A Comprehensive Review. Diagnostics (Basel) 2015;5:119-176.

157. Yamaguchi T, Machida N, Morizane C, et al. Multicenter retrospective analysis of systemic chemotherapy for advanced neuroendocrine carcinoma of the digestive system. Cancer Sci 2014;105:1176-1181.

158. Garcia-Carbonero R, Sorbye H, Baudin E, et al. ENETS Consensus Guidelines for High-Grade Gastroenteropancreatic Neuroendocrine Tumors and Neuroendocrine Carcinomas. Neuroendocrinology 2016;103:186-194.

159. Terashima T, Morizane C, Hiraoka N, et al. Comparison of chemotherapeutic treatment outcomes of advanced extrapulmonary neuroendocrine carcinomas and advanced small-cell lung carcinoma. Neuroendocrinology 2012;96:324-332.

160. Grivas P, Bismar TA, Alva AS, et al. Validation of a neuroendocrine-like classifier confirms poor outcomes in patients with bladder cancer treated with cisplatin-based neoadjuvant chemotherapy. Urologic oncology 2020;38:262-268.

161. Bergsland EK, Roy R, Stephens P, et al. Genomic profiling to distinguish poorly differentiated neuroendocrine carcinomas arising in different sites. Journal of Clinical Oncology 2016;34:4020-4020.

162. Hijioka S, Hosoda W, Matsuo K, et al. Rb Loss and KRAS Mutation Are Predictors of the Response to Platinum-Based Chemotherapy in Pancreatic Neuroendocrine Neoplasm with Grade 3: A Japanese Multicenter Pancreatic NEN-G3 Study. Clin Cancer Res 2017;23:4625-4632.

163. Rekhtman N, Desmeules P, Litvak AM, et al. Stage IV lung carcinoids: spectrum and evolution of proliferation rate, focusing on variants with elevated proliferation indices. Mod Pathol 2019.

164. Swarts DR, Ramaekers FC, Speel EJ. Molecular and cellular biology of neuroendocrine lung tumors: evidence for separate biological entities. Biochimica et biophysica acta 2012;1826:255-271.

165. Caplin ME, Baudin E, Ferolla P, et al. Pulmonary neuroendocrine (carcinoid) tumors: European Neuroendocrine Tumor Society expert consensus and recommendations for best practice for typical and atypical pulmonary carcinoids. Ann Oncol 2015;26:1604-1620.

166. Alcala N, Leblay N, Gabriel AAG, et al. Integrative and comparative genomic analyses identify clinically relevant pulmonary carcinoid groups and unveil the supra-carcinoids. Nat Commun 2019;10:3407.

167. Pelosi G, Bianchi F, Dama E, et al. Most high-grade neuroendocrine tumours of the lung are likely to secondarily develop from pre-existing carcinoids: innovative findings skipping the current pathogenesis paradigm. Virchows Arch 2018;472:567-577.

168. Moonen L, Derks J, Dingemans AM, et al. Orthopedia Homeobox (OTP) in Pulmonary Neuroendocrine Tumors: The Diagnostic Value and Possible Molecular Interactions. Cancers 2019;11. 

Addendum

Summary 



\section{Summary}

Pulmonary neuroendocrine neoplasms (NEN) are subdivided in well differentiated typical and atypical carcinoids (TC and $A C$ ) and poorly differentiated neuroendocrine carcinomas (NEC: small cell lung carcinoma (SCLC) and large cell neuroendocrine carcinoma (LCNEC)) (chapter 1). ${ }^{1,2}$ LCNEC represents approximately $1-3 \%$ of new lung cancer cases. ${ }^{3-6}$ LCNEC is characterized by neuroendocrine morphology and large cells with a moderate to abundant amount of cytoplasm and presence of nucleoli. ${ }^{2}$ Furthermore, immunohistochemical (IHC) expression of at least one neuroendocrine marker (Synaptophysin, Chromogranin A or Neural Cell Adhesion Molecule 1 (Ncam1, Cd56); $>10 \%$ of the tumor), presence of necrosis and a mitotic index (MI) $>10 / 2 \mathrm{~mm}^{2}$ are required to confirm LCNEC diagnosis. $^{2}$ So far, at least two exclusive molecular subtypes of LCNEC have been identified by next generation sequencing. The first is a SCLC-like type, with co-mutation of RB1 and TP53 and loss of IHC pRb expression. The second is a NSCLC-like type, with co-mutation of TP53 and STK11/KEAP1 or KRAS genes and preserved $\mathrm{pRb}$ expression. ${ }^{7-9}$ These molecular patterns might be predictive for chemotherapeutic responses. ${ }^{7,10}$ In about half of the cases, LCNEC patients present with metastatic disease at diagnosis. ${ }^{3,5,6,11,12}$ Median overall survival is $12-32$ months for stage I-III patients, and only 4-9 months in patients with stage IV disease., ${ }^{3,6,12,13}$ Only few studies have evaluated optimal treatment strategies for LCNEC and knowledge extrapolated from NSCLC and/or SCLC is usually applied to guide treatment protocols. In case of localized disease, anatomical resection minimally by lobectomy is recommended, supplemented with adjuvant chemotherapy similar to NSCLC disease (i.e. at least in stage II and III (TNM8)). ${ }^{14-21}$ For stage IV, palliative chemotherapy with both a SCLC regimen (cisplatin/carboplatin + etoposide) and NSCLC regimen (platinum + gemcitabin/taxane) are deemed appropriate. ${ }^{22}$ In the last decades, treatment regimens have not substantially evolved. However, in recent years, immunotherapy and targeted therapy have received attention as potential treatment strategies for LCNEC. Furthermore, subtypes with aberrant clinical behavior compared to general LCNEC have been recognized, with a possible impact on prognosis and treatment regimens.

The aim of this thesis was to obtain a deeper insight into relevant molecular and clinical subtypes of LCNEC. Predictive and prognostic markers within LCNEC subtypes were investigated using imaging features, next generation sequencing and histopathological evaluation (e.g. Ki-67, programmed-death ligand 1 (Pd-I1) and delta like ligand 3 (DII3)) to asses prognosis and guide optimal treatment strategies for individual LCNEC patients. Furthermore, clinically relevant differences and similarities between LCNEC and NEN of other primary origins were evaluated in a nationwide database. 


\section{Imaging features to differentiate between molecular LCNEC subtypes}

In chapter 2, radiological features of LCNEC and the possibility to discriminate molecular SCLC-like and NSCLC-like LCNEC subtypes based on radiological imaging were evaluated. The aim was to find a less invasive alternative for (repeated) biopsies to subclassify LCNEC in cases where surgical specimens are not available. Three evaluation methods were used: 1) Interpretation of SCLC-like or NSCLC-like appearance of LCNEC tumors on computed tomography (CT)-scans by pulmonary oncologists and radiologists; 2) assessment of semantic features of LCNEC CT-scans by radiologists; 3 ) application of a radiomics signature, developed to separate SCLC from NSCLC, on molecular LCNEC subtypes. Pulmonary oncologists and chest radiologists assessed chest CT-scans of 44 LCNEC patients for 'small cell-like' or 'non-small cell-like' appearance. The radiologists also scored semantic features of 50 LCNEC scans. A radiomics signature was trained on a dataset containing 48 SCLC and 76 NSCLC scans and validated on an external set of 58 SCLC and 40 NSCLC scans and this signature was applied on scans of 28 SCLC-like and 8 NSCLC-like LCNEC patients. The pulmonary oncologists and radiologists were unable to differentiate between molecular subtypes of LCNEC. Although some semantic features were observed in LCNEC scans in a comparable percentage to that in SCLC or NSCLC scans, most semantic features in LCNEC were identified in percentages in between SCLC and NSCLC. However, no significant differences in semantic features were found between molecular LCNEC subtypes. External validation of the radiomics signature showed a good performance to separate SCLC from NSCLC (area under the curve (AUC) 0.84 (95\% confidence interval (CI) 0.77 $0.92)$ ). Nonetheless, this signature could not identify SCLC-like and NSCLC-like LCNEC (AUC 0.58 (95\% Cl 0.29-0.86). Remarkably, most LCNEC were classified by clinicians and radiomics as NSCLC-like despite they had SCLC-like molecular characteristics. These results indicate that, based on their unique imaging characteristics compared to SCLC and NSCLC, LCNEC can be considered a unique tumor entity.

\section{LCNEC subtypes with specific histopathological or clinical features}

\subsection{Combined LCNEC-adenocarcinoma and LCNEC with an ipsilateral co-primary adenocarcinoma}

LCNEC may present in combination with other NSCLC, e.g. adenocarcinoma (ADC) or squamous cell carcinoma. LCNEC with ADC may arise both as a continuity (combined tumors) and as multiple synchronous ipsilateral lesions (co-primary). Molecular and histopathological analysis of both tumor parts could give additional insight in oncogenesis of those tumors and is provided in chapter 3. In all 10 identified combined 
tumors, the LCNEC- and ADC-parts were clonally related. A high rate of mutations frequently encountered in pure $A D C$ was found, but $p R b$ inactivation, associated with neuroendocrine differentiation, was also seen more often than expected in ADC. Some neuroendocrine differentiation (i.e. IHC expression of neuroendocrine markers, preserved morphology) in ADC-parts of the combined tumors reflected developing neuroendocrine activity in those parts. Furthermore, an increase in Ascl1 expression and decrease in Rest expression in neuroendocrine parts indicated a role for these regulators in neuroendocrine differentiation. Of the 5 co-primary LCNEC and ADC tumors, only 1 set was clonally related, implying that these tumors in general should be regarded as two primary lesions instead of metastatic disease.

\subsection{LCNEC with a solitary brain metastasis}

LCNEC patients present with metastatic disease at diagnosis in about half of the cases. Mostly, this is disseminated metastatic disease. However, in chapter 4, 11 patients with only a solitary brain metastasis diagnosed as LCNEC were identified. Clinical and histopathological characteristics of these cases were evaluated. The Ki- 67 proliferation index (Ki-67 PI) was identified to be of potential prognostic relevance in this subtype. In 6/11 cases tumor Ki-67 PI was $\leq 40 \%$ and overall survival was longer compared to cases with tumor Ki-67 PI >40\% (17 months ( $95 \%$ Cl 11-23 months) vs. 5 months (95\% Cl 0.7-9 months), $\mathrm{p}=0.007$ ). Two patients with Ki-67 $\leq 40 \%$ even had long term survival, and were still alive at follow-up after 86 and 103 months. Patients within this subtype (solitary brain metastasis, Ki-67 PI $\leq 40 \%$ ) might benefit from more aggressive and even definitive therapy, instead of palliative chemotherapy. This study emphasized that LCNEC is a heterogeneous type of cancer and underscored the importance to identify different subtypes of LCNEC.

\subsection{LCNEC with well differentiated morphology}

Another possible clinical LCNEC subtype consists of tumors classifying as LCNEC because of high proliferation rate as defined by $\mathrm{MI}$ and/or $\mathrm{Ki}-67 \mathrm{PI}$, but also showing a well differentiated morphology. In the WHO classification of gastro-intestinal and pancreatic NEN, such tumors are classified as grade 3 NET, instead of NEC. In chapter $\mathbf{5}$, an overview is provided of the limited literature available considering this kind of patients in pulmonary NEN. Furthermore, 7 additional cases were described and a remarkably longer than expected median overall survival in stage IV cases with preserved $\mathrm{pRb}$ expression was found, compared to general LCNEC (45 vs. 4-9 months). Despite the indications for prognostic relevance, clinical relevance including progression free 
survival and optimal treatment regimens (i.e. comparable to carcinoid vs. NEC regimens) remain to be studied for this subtype.

\section{Markers for systemic treatment}

\subsection{DII3 as a potential therapeutic target for treatment of neuroendocrine carcinoma}

Recently, DII3 has been proposed as a therapeutic target for NEC. In chapter 6, current literature on DII3 expression in NEC (both SCLC and LCNEC) and possible treatment options were reviewed. DII3 is expressed in 64-90\% of SCLC and LCNEC, whereas no or only very limited expression is observed in normal tissue. Currently, three different approaches using Antibody-Drug Conjugates (ADC), Bispecific T-cell Engaging antibodies $\left(\right.$ BiTE $^{\circledR}$ ) and Chimeric Antigen Receptor T-cells (CAR-T) are in development for Dll3 targeted therapy. Development of the ADC rovalpituzumab-tesirine has been halted after early termination of two phase III studies. Efficacy and safety of BiTE ${ }^{\circledR} \mathrm{S}$ and CAR-T cells still have to be demonstrated and phase I trials are running.

Chapter $\mathbf{7}$ added to existing literature by the investigation of DII3 expression in stage IV LCNEC. DII3 was expressed in 70/94 (74\%) LCNEC, 56 (80\%) of which showed cytoplasmic/ membranous staining. DII3 staining was not different in pRb IHC negative and positive patients (DII3+ in 53/70 (76\%) vs. 14/21 (67\%), $p=0.409$ ). Nevertheless, 6/6 (100\%) STK11 mutated vs. 44/61 (72\%) STK11 wildtype $(p=0.33)$ and 10/11 (91\%) KEAP1 mutated vs. 40/56 (71\%) KEAP1 wildtype tumors $(p=0.27)$ were DII3 positive. Furthermore, DII3 expression was associated with expression of Ascl1 and at least 2 out of 3 neuroendocrine markers. Altogether, our data and literature review revealed that DII3 is a promising therapeutic target for SCLC and LCNEC, but further development of potential compounds and trials to reveal their safety and effectivity are necessary.

\subsection{Pd-I1 expression in LCNEC as an indication for response to Pd-(I)1 targeted therapy}

Based on positive clinical effects in other types of lung cancer and the high mutational burden of LCNEC, Pd-(I)1 targeted therapy could be a new treatment option in (metastatic) LCNEC. Previous studies, mainly in resected cases with non-metastatic LCNEC, reported Pd-I1 expression in 9-32\% of LCNEC. In chapter 8, Pd-I1 expression in 98 stage IV LCNEC was evaluated and expression was found in $16 \%$ of cases. This expression was not related to SCLC-like or NSCLC-like molecular subtypes, but it was related to $\mathrm{Cd} 8$ expressing cells in the tumor. Furthermore, only a limited number of tumors had $>1 \%$ intra-tumor $\mathrm{Cd} 8$ staining, whereas $\mathrm{Cd} 8$ expression was present in stromal cells in the majority of cases. This indicates that most LCNEC are 'immune excluded', making it difficult for T-cells to invade the tumor. The low number of stage IV 
LCNEC tumors positive for Pd-I1 questions the role of single agent Pd-(I)1 inhibition in metastatic LCNEC and calls for combination strategies.

\section{Metastatic patterns in neuroendocrine neoplasms of the lung and other primary origins}

In chapter 9, insight in similarities and differences between NEN of different primary origins is provided by comparing metastatic patterns in pulmonary NEN with metastatic patterns in gastroenteral and pancreatic NEN in a nationwide cohort of 11,120 patients. About half of the patients presented with metastatic disease. In gastroenteral and pancreatic NEN, liver metastases were most frequent $(25 \%$ and $39 \%$ of all new NEN cases, respectively), whereas in pulmonary NEN the prevalence of metastases was scattered with metastases in the liver (19\%), brain (9\%), lung (7\%) and bone (14\%). Furthermore, at time of presentation, brain metastases were almost absent in gastroenteral and pancreatic NEN, in contrast to the higher frequency in pulmonary NEN (especially LCNEC). Therefore, screening for brain metastases might be considered in pulmonary NEN, whereas it seems not to be useful in NEN from other primary origins. To secure optimal treatment for all unique NEN patients it is essential to increase awareness for these differences among clinicians treating both gastrointestinal and pulmonary NEN.

\section{Discussion}

Finally, in chapter 10, a general discussion on results obtained in this thesis and current literature available on LCNEC is provided. Suggestions for future research are given, e.g. to explore a new molecular subclassification in LCNEC, the use of new methods for subclassification, and analysis of potential prognostic and predictive markers. Finally, limitations of the current WHO classification for pulmonary NEN are considered and LCNEC is suggested to be part of a neuroendocrine spectrum with overlap with other (neuroendocrine) pulmonary tumors and including clinically relevant subtypes. To conclude, a more balanced way between application of the clear diagnostic WHO criteria and awareness for subtypes, and prognostic and predictive factors will aid the optimal approach to all unique pulmonary NEN patients. 


\section{References}

1. Rindi G, Klimstra DS, Abedi-Ardekani B, et al. A common classification framework for neuroendocrine neoplasms: an International Agency for Research on Cancer (IARC) and World Health Organization (WHO) expert consensus proposal. Mod Pathol 2018;31:1770-1786.

2. Travis WD, Brambilla E, Burke AP, et al. WHO Classification of Tumours of the Lung, Pleura, Thymus and Heart Lyon, France: International Agency for Research on Cancer; 2015.

3. Derks JL, Hendriks LE, Buikhuisen WA, et al. Clinical features of large cell neuroendocrine carcinoma: a population-based overview. Eur Respir J 2016;47:615-624.

4. Takei $\mathrm{H}$, Asamura $\mathrm{H}$, Maeshima $\mathrm{A}$, et al. Large cell neuroendocrine carcinoma of the lung: a clinicopathologic study of eighty-seven cases. J Thorac Cardiovasc Surg 2002;124:285-292.

5. Korse CM, Taal BG, van Velthuysen ML, et al. Incidence and survival of neuroendocrine tumours in the Netherlands according to histological grade: experience of two decades of cancer registry. Eur J Cancer 2013;49:1975-1983.

6. Varlotto JM, Medford-Davis LN, Recht A, et al. Should large cell neuroendocrine lung carcinoma be classified and treated as a small cell lung cancer or with other large cell carcinomas? J Thorac Oncol 2011;6:1050-1058.

7. Derks JL, Leblay N, Thunnissen E, et al. Molecular Subtypes of Pulmonary Large-cell Neuroendocrine Carcinoma Predict Chemotherapy Treatment Outcome. Clin Cancer Res 2018;24:33-42.

8. George J, Walter V, Peifer $\mathrm{M}$, et al. Integrative genomic profiling of large-cell neuroendocrine carcinomas reveals distinct subtypes of high-grade neuroendocrine lung tumors. Nat Commun 2018;9:1048.

9. Rekhtman N, Pietanza MC, Hellmann MD, et al. Next-Generation Sequencing of Pulmonary Large Cell Neuroendocrine Carcinoma Reveals Small Cell Carcinoma-like and Non-Small Cell Carcinoma-like Subsets. Clin Cancer Res 2016;22:3618-3629.

10. Zhou F, Hou L, Ding T, et al. Distinct clinicopathologic features, genomic characteristics and survival of central and peripheral pulmonary large cell neuroendocrine carcinoma: From different origin cells? Lung Cancer 2018;116:30-37.

11. Grondahl V, Binderup T, Langer SW, et al. Characteristics of 252 patients with bronchopulmonary neuroendocrine tumours treated at the Copenhagen NET Centre of Excellence. Lung Cancer 2019;132:141-149.

12. Cao L, Li ZW, Wang M, et al. Clinicopathological characteristics, treatment and survival of pulmonary large cell neuroendocrine carcinoma: a SEER population-based study. PeerJ 2019;7:e6539.

13. Asamura $\mathrm{H}$, Kameya $\mathrm{T}$, Matsuno $\mathrm{Y}$, et al. Neuroendocrine neoplasms of the lung: a prognostic spectrum. J Clin Oncol 2006;24:70-76.

14. Jiang $Y$, Lei $C$, Zhang $X$, et al. Double-edged role of radiotherapy in patients with pulmonary large-cell neuroendocrine carcinoma. J Cancer 2019;10:6422-6430.

15. Wakeam E, Adibfar A, Stokes S, et al. Defining the role of adjuvant therapy for early-stage large cell neuroendocrine carcinoma. J Thorac Cardiovasc Surg 2019.

16. Ogawa $\mathrm{H}$, Tanaka $\mathrm{Y}$, Kitamura $\mathrm{Y}$, et al. Efficacy of perioperative chemotherapy for pulmonary high-grade neuroendocrine carcinomas: a propensity score matching analysis. J Thorac Dis 2019;11:1145-1154.

17. Tsoukalas N, Baxevanos P, Aravantinou-Fatorou E, et al. Advances on systemic treatment for lung neuroendocrine neoplasms. Ann Transl Med 2018;6:146.

18. Filosso PL, Guerrera F, Evangelista A, et al. Adjuvant chemotherapy for large-cell neuroendocrine lung carcinoma: results from the European Society for Thoracic Surgeons Lung Neuroendocrine Tumours Retrospective Database. Eur J Cardiothorac Surg 2017;52:339-345.

19. Iyoda A, Hiroshima K, Moriya Y, et al. Prospective study of adjuvant chemotherapy for pulmonary large cell neuroendocrine carcinoma. Ann Thorac Surg 2006;82:1802-1807.

20. Kenmotsu $\mathrm{H}$, Niho $\mathrm{S}$, Ito $\mathrm{T}$, et al. A pilot study of adjuvant chemotherapy with irinotecan and cisplatin for completely resected high-grade pulmonary neuroendocrine carcinoma (large cell neuroendocrine carcinoma and small cell lung cancer). Lung Cancer 2014;84:254-258. 
21. Rossi G, Cavazza A, Marchioni A, et al. Role of chemotherapy and the receptor tyrosine kinases KIT, PDGFRalpha, PDGFRbeta, and Met in large-cell neuroendocrine carcinoma of the lung. J Clin Oncol 2005;23:8774-8785.

22. Hanna N, Johnson D, Temin S, et al. Systemic Therapy for Stage IV Non-Small-Cell Lung Cancer: American Society of Clinical Oncology Clinical Practice Guideline Update. J Clin Oncol 2017;35: 3484-3515. 

Samenvatting 



\section{Samenvatting}

Pulmonale neuro-endocriene neoplasmata (NEN) zijn onderverdeeld in goed gedifferentieerde tumoren (typisch carcinoïd (TC) en atypisch carcinoïd (AC) en slecht gedifferentieerde carcinomen (NEC: kleincellig longcarcinoom (SCLC) en grootcellig neuro-endocrien carcinoom (LCNEC)) (hoofdstuk 1). ${ }^{1,2} \mathrm{NEN}$ is een zeldzame maligniteit

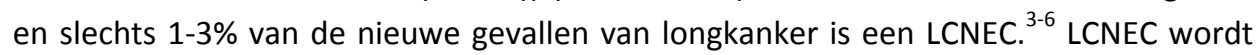
gekenmerkt door een neuro-endocriene morfologie en grote cellen met een matige tot grote hoeveelheid cytoplasma en de aanwezigheid van nucleoli. ${ }^{2}$ Bovendien zijn immunohistochemische (IHC) expressie van ten minste één neuro-endocriene marker (Synaptofysine, Chromogranine A of Neurale celadhesiemolecule 1 (Ncam1, Cd56); $>10 \%$ van de tumor), aanwezigheid van necrose en een mitotische index (MI) $>10 / 2 \mathrm{~mm}^{2}$ vereist om de diagnose LCNEC te stellen. ${ }^{2}$ De diagnostiek op basis van een biopt wordt bemoeilijkt, doordat hierbij de neuro-endocriene morfologie niet altijd goed te herkennen is. Tot dusver zijn er ten minste twee exclusieve moleculaire subtypen van LCNEC geïdentificeerd door middel van next-generation sequencing. De eerste is een SCLC-achtig type, met co-mutatie van RB1 en TP53 en verlies van IHC pRb expressie. De tweede is een niet-kleincellig longcarcinoom (NSCLC)-achtig type, met comutatie van de genen TP53 en STK11/KEAP1 of KRAS en een behouden pRb expressie. $^{7-9}$ Deze moleculaire patronen kunnen voorspellend zijn voor de respons op chemotherapie. ${ }^{7,10}$ In ongeveer de helft van de gevallen hebben patiënten met LCNEC gemetastaseerde ziekte op het moment van diagnose. ${ }^{3,5,6,11,12}$ De mediane totale overleving is 12-32 maanden voor stadium I-III patiënten en slechts 4-9 maanden voor patiënten met stadium IV ziekte. ${ }^{3,6,12,13}$ Bij gelokaliseerde ziekte wordt anatomische resectie met minimaal een lobectomie aanbevolen, eventueel aangevuld met adjuvante chemotherapie zoals dit ook voor NSCLC wordt gedaan (d.w.z. ten minste bij stadium II en III (TNM8)). ${ }^{14-21}$ Door de zeldzaamheid van de ziekte en de beperkte mogelijkheid om de diagnose op een biopt te stellen, is de optimale behandelstrategie van stadium IV ziekte slechts beperkt onderzocht. Veelal wordt kennis die is opgedaan bij de behandeling van patiënten met NSCLC en/of SCLC gebruikt om behandelprotocollen voor LCNEC op te stellen. Voor stadium IV wordt palliatieve chemotherapie met zowel een SCLC-schema (cisplatine/carboplatine + etoposide) als een NSCLC-schema (platinum bevattende chemotherapie + gemcitabine/taxaan) geschikt geacht. ${ }^{22}$ In de afgelopen decennia zijn er geen duidelijke verbeteringen geweest in de behandelmogelijkheden voor stadium IV LCNEC. In de afgelopen jaren zijn immunotherapie en doelgerichte therapie echter geopperd als mogelijke behandelingsstrategieën. Bovendien zijn er subtypen met een afwijkend klinisch gedrag ten opzichte 
van de gemiddelde LCNEC tumor gevonden. Deze subtypen hebben mogelijk impact op de prognose en behandelopties van specifieke patiënten.

Het doel van dit proefschrift was om meer inzicht te krijgen in relevante moleculaire en klinische subtypen van LCNEC. Door evaluatie van radiologische en histopathologische kenmerken (bijvoorbeeld Ki-67, programmed-death ligand 1 (Pd-I1) en delta-like ligand 3 (DII3)) en next generation sequencing werden predictieve en prognostische markers onderzocht binnen de LCNEC subtypen met als doel een betere voorspelling van de prognose te geven en behandelopties te optimaliseren voor individuele LCNEC patiënten. Bovendien werden klinisch relevante verschillen en overeenkomsten tussen LCNEC en NEN van andere primaire origine onderzocht in een landelijke database.

\section{Het gebruik van radiologische kenmerken om onderscheid te maken tussen moleculaire subtypen van LCNEC}

In hoofdstuk 2 worden radiologische kenmerken van LCNEC beschreven en is tevens onderzocht of de SCLC-achtige en NSCLC-achtige LCNEC subtypen te onderscheiden zijn op basis van radiologische beeldvorming. Dit had als doel een minder invasief alternatief te vinden voor (herhaalde) biopsieën om LCNEC te subclassificeren in gevallen waarin geen chirurgisch materiaal beschikbaar is. Er werden drie methoden gebruikt: 1) Beoordeling door longartsen met aandachtsgebied oncologie en radiologen van computertomografie (CT)-scans van LCNEC, waarbij een interpretatie van SCLCachtige of NSCLC-achtige kenmerken gegeven werd; 2) beoordeling door radiologen van semantische kenmerken van LCNEC tumoren op CT-scans; 3 ) toepassing van een radiomics signatuur, ontwikkeld om SCLC en NSCLC te onderscheiden, op de moleculaire LCNEC subtypen. De longartsen en radiologen waren niet in staat om onderscheid te maken tussen moleculaire subtypen van LCNEC. Hoewel sommige semantische kenmerken in een vergelijkbaar percentage werden gevonden bij LCNEC scans als bij SCLC- of NSCLC scans, werden de meeste semantische kenmerken bij LCNEC geïdentificeerd in een percentage van de scans tussen SCLC en NSCLC in. Er werden echter geen significante verschillen in semantische kenmerken gevonden tussen de moleculaire LCNEC subtypen. Externe validatie van de radiomics signatuur toonde dat deze geschikt is om SCLC te onderscheiden van NSCLC (area under the curve (AUC) 0.84 (95\% betrouwbaarheidsinterval (BI) 0.77-0.92)). Met deze signatuur konden de SCLC-achtige en NSCLC-achtige LCNEC echter niet geïdentificeerd worden (AUC 0.58 (95\% BI 0.29-0.86). Opmerkelijk is dat de meeste LCNEC op basis van de beeldvorming door de clinici en de radiomics signatuur werden geclassificeerd als NSCLC-achtig terwijl de meesten tot het moleculaire SCLC-achtige subtype behoorden. Op basis van deze 
unieke radiologische kenmerken in vergelijking met SCLC en NSCLC kan LCNEC worden beschouwd als een afzonderlijke tumorsoort.

\section{LCNEC subtypen met specifieke histopathologische of klinische kenmerken}

\subsection{Gecombineerd LCNEC-adenocarcinoom en LCNEC met een ipsilateraal co-primair adenocarcinoom}

LCNEC kan aanwezig zijn in combinatie met een andere vorm van NSCLC, bijvoorbeeld het adenocarcinoom of plaveiselcelcarcinoom. LCNEC in combinatie met adenocarcinoom kan als een continuüm (gecombineerde tumoren) of als multiple synchrone ipsilaterale laesies (co-primair) optreden. Moleculaire en histopathologische analyse van beide tumordelen kan aanvullend inzicht geven in de oncogenese van deze tumoren. In alle 10 geïdentificeerde gecombineerde tumoren in hoofdstuk $\mathbf{3}$ waren de LCNEC- en adenocarcinoom-delen klonaal gerelateerd. Er werd een hoog aantal mutaties gevonden die frequent worden gezien bij een puur adenocarcinoom. Ook pRb inactivatie, geassocieerd met neuro-endocriene differentiatie, werd vaker gezien dan verwacht. De ontwikkeling van neuro-endocriene activiteit in adenocarcinoom-delen van de gecombineerde tumoren werd weerspiegeld door een beginnende neuroendocriene differentiatie (d.w.z. IHC expressie van neuro-endocriene markers, behouden morfologie) in die delen. Bovendien duidden een toename in Ascl1 expressie en afname in Rest expressie in neuro-endocriene delen op een rol voor deze regulatoren bij de neuro-endocriene differentiatie. Van de 5 co-primaire LCNEC- en adenocarcinoom-tumoren was slechts 1 set klonaal gerelateerd, wat impliceert dat deze tumoren in het algemeen als twee primaire laesies moeten worden beschouwd en niet als metastatische ziekte.

\subsection{LCNEC met een solitaire hersenmetastase}

Patiënten met LCNEC presenteren zich bij diagnose in ongeveer de helft van de gevallen met gemetastaseerde ziekte. Meestal is er sprake van uitgebreide metastasering. In hoofdstuk 4 werden echter 11 patiënten geïdentificeerd met alleen een solitaire hersenmetastase. De klinische en histopathologische kenmerken van deze cases werden geëvalueerd. De Ki-67 proliferatie-index (Ki-67 PI) bleek potentieel prognostisch relevant te zijn in dit subtype. In 6/11 gevallen was de tumor Ki-67 PI $\leq 40 \%$ en in deze groep was de algehele overleving langer dan de overleving in de groep met Ki-67 PI> 40\% (17 maanden (95\% BI 11-23 maanden) vs. 5 maanden (95\% BI 0.7-9 maanden), $p=0.007)$. Twee patiënten met $\mathrm{Ki}-67 \leq 40 \%$ hadden zelfs een langdurige 
overleving en waren bij follow-up na 86 en 103 maanden nog in leven. Patiënten binnen dit subtype van LCNEC met solitaire hersenmetastasen en Ki-67 PI $\leq 40 \%$ zouden baat kunnen hebben bij agressievere en zelfs definitieve therapie in plaats van palliatieve chemotherapie. Deze studie benadrukt het heterogene karakter van LCNEC en toont het belang om verschillende subtypes van LCNEC te identificeren.

\subsection{LCNEC met goed gedifferentieerde morfologie}

Een ander mogelijk klinisch relevant LCNEC subtype bestaat uit tumoren die als LCNEC worden geclassificeerd vanwege een hoge proliferatie (gedefinieerd door de $\mathrm{MI}$ en/of Ki-67 PI), maar een goed gedifferentieerde morfologie hebben. In de WHO-classificatie van gastro-intestinale en pancreas NEN worden dergelijke tumoren geclassificeerd als graad 3 neuro-endocriene tumoren (NET), in plaats van als NEC. In hoofdstuk 5 wordt een overzicht gegeven van de beperkte literatuur die beschikbaar is over dit soort patiënten met pulmonale NEN. Tevens worden 7 aanvullende cases beschreven. In de stadium IV cases met behouden pRb-expressie werd een opmerkelijk langere dan verwachte mediane totale overleving gevonden in vergelijking met LCNEC in het algemeen (45 vs. 4-9 maanden). Ondanks deze suggestie voor prognostische relevantie moet de klinische relevantie nog onderzocht worden voor dit subtype met hierbij ook aandacht voor progressievrije overleving en optimale behandelingsstrategieën (d.w.z. behandeling conform carcinoïd-behandeling danwel NEC-behandeling).

\section{Markers voor systemische behandeling}

3.1 Dll3 als potentieel therapeutisch doelwit voor de behandeling van het neuroendocrien carcinoom

Onlangs is DIl3 voorgesteld als een therapeutisch doelwit voor gerichte behandeling van NEC. In hoofdstuk 6 zijn de huidige literatuur over DIl3 expressie in NEC (zowel SCLC als LCNEC) en mogelijke behandelopties besproken. Dll3 wordt gevonden in 6490\% van SCLC en LCNEC, terwijl er geen of slechts een zeer beperkte expressie wordt gezien in normaal weefsel. Momenteel zijn er drie verschillende methoden in ontwikkeling voor Dll3 gerichte therapie: Antibody-Drug Conjugates (ADC), Bispecific T-cell Engaging Antodies $\left(\mathrm{BiTE}^{\circledR}\right)$ en Chimeric Antigen Receptor T-cells (CAR-T). De ontwikkeling van het $A D C$ rovalpituzumab-tesirine is gestopt na vroegtijdige beëindiging van twee fase III onderzoeken. De werkzaamheid en veiligheid van BiTEs $^{\circledR}$ en CAR-T-cellen moeten nog worden aangetoond en er lopen fase I onderzoeken.

In aanvulling op de reeds bestaande literatuur wordt in hoofdstuk 7 DII3 expressie in stadium IV LCNEC beschreven. DII3 kwam tot expressie in 70/94 (74\%) LCNEC, waarvan 
56 cases (80\%) een cytoplasmatische/membraneuze kleuring vertoonden. DII3 expressie was niet verschillend tussen $\mathrm{pRb}$ IHC negatieve of positieve patiënten (DII3+ in 53/70 (76\%) vs. $14 / 21$ (67\%), $\mathrm{p}=0.409)$. Desalniettemin waren $6 / 6$ (100\%) STK11 gemuteerde vs. $44 / 61$ (72\%) STK11 wildtype $(p=0.33)$ en 10/11 (91\%) KEAP1 gemuteerde vs. $40 / 56$ (71\%) KEAP1 wildtype tumoren ( $p=0.27$ ) Dll3 positief. Bovendien was DII3 expressie geassocieerd met expressie van Ascl1 en ten minste 2 van de 3 neuro-endocriene markers. Al met al tonen onze gegevens en literatuuronderzoek dat DII3 een veelbelovend therapeutisch doelwit is voor SCLC en LCNEC. Verdere ontwikkeling van potentiële middelen en onderzoeken om hun veiligheid en effectiviteit te bepalen zijn echter nog noodzakelijk voordat deze doelgerichte therapie toegepast kan worden.

\subsection{Pd-I1 expressie in LCNEC als een indicatie voor respons op Pd-(I)1 gerichte therapie}

Op basis van positieve klinische effecten bij andere typen longkanker en het hoge aantal mutaties bij LCNEC, zou therapie gericht op Pd-(I)1 een nieuwe behandeloptie kunnen zijn voor (gemetastaseerde) LCNEC. Eerdere studies toonden Pd-I1 expressie in 9-32\% van voornamelijk niet-gemetastaseerde, gereseceerde LCNEC. In hoofdstuk 8 werd Pd-I1 expressie in 98 stadium IV LCNEC geëvalueerd en expressie $\geq 1 \%$ werd gevonden in slechts $16 \%$ van de gevallen. Deze expressie was niet gerelateerd aan het SCLC-achtige of NSCLC-achtige moleculaire subtype, maar wel aan tumor-infiltrerende cellen die $\mathrm{Cd} 8$ tot expressie brengen. Bovendien had een beperkt aantal tumoren een intra-tumor Cd8 expressie $>1 \%$, terwijl Cd8 kleuring in de meeste gevallen wel aanwezig was in de stromale cellen. Dit geeft aan dat de meeste LCNEC 'immune excluded' zijn, waarbij het voor T-cellen moeilijk is om de tumor binnen te dringen. Hierdoor en door het lage aantal stadium IV LCNEC tumoren dat positief is voor $\mathrm{Pd}-\mathrm{I} 1$, is het onwaarschijnlijk dat $\mathrm{Pd}-(\mathrm{I}) 1$ inhibitie een rol kan hebben als monotherapie. Van combinatie therapieën waar $\mathrm{Pd}-(\mathrm{I}) 1$ inhibitoren onderdeel van uitmaken, is mogelijk meer te verwachten.

\section{Metastatische patronen in neuro-endocriene neoplasmata van de long en andere primaire origine}

In hoofdstuk 9 wordt inzicht gegeven in overeenkomsten en verschillen tussen NEN van verschillende primaire origine door in een landelijk cohort van 11.120 patiënten uitzaaiingspatronen in pulmonale NEN te vergelijken met uitzaaiingspatronen in gastroenterale en pancreas NEN. Ongeveer de helft van de patiënten had gemetastaseerde ziekte bij diagnose. In gastro-enterale en pancreas NEN kwamen levermetastasen het meest voor (respectievelijk $25 \%$ en $39 \%$ van alle nieuwe NEN), terwijl bij pulmonale 
NEN de prevalentie van metastasen in verschillende organen meer gelijk verdeeld was met uitzaaiingen in de lever (19\%), hersenen (9\%), longen (7\%) en botten (14\%). Bovendien waren hersenmetastasen op het moment van presentatie vrijwel afwezig in gastro-enterale en pancreas NEN, terwijl hiervan bij pulmonale NEN (met name LCNEC) een hogere incidentie werd gevonden. Screening op hersenmetastasen zou daarom overwogen kunnen worden in pulmonale NEN, terwijl het niet nuttig lijkt te zijn in NEN van andere primaire origine. Een toegenomen bewustzijn over deze verschillen bij clinici die zowel gastro-enterale als pulmonale NEN behandelen is essentieel voor een optimale behandeling van alle unieke NEN patiënten.

\section{Discussie}

Ten slotte wordt in hoofdstuk 10 een algemene discussie gegeven over de resultaten die in dit proefschrift zijn beschreven en de huidige beschikbare literatuur over LCNEC. Er worden suggesties gegeven voor toekomstig onderzoek, bijvoorbeeld een nieuwe moleculaire subclassificatie van LCNEC, het gebruik van nieuwe methoden voor subclassificatie en analyse van potentiële prognostische en predictieve markers. Ten slotte worden de beperkingen van de huidige WHO-classificatie voor pulmonale NEN besproken en wordt gesuggereerd dat LCNEC deel uitmaakt van een neuro-endocrien spectrum met overlap met andere (neuro-endocriene) pulmonale tumoren en aanwezigheid van klinisch relevante subtypen. Concluderend kan de benadering van patiënten met pulmonale NEN worden geoptimaliseerd door een meer gebalanceerde manier van toepassing van WHO-criteria en bewustwording van subtypes en prognostische en predictieve markers. 


\section{Referenties}

1. Rindi G, Klimstra DS, Abedi-Ardekani B, et al. A common classification framework for neuroendocrine neoplasms: an International Agency for Research on Cancer (IARC) and World Health Organization (WHO) expert consensus proposal. Mod Pathol 2018;31:1770-1786.

2. Travis WD, Brambilla E, Burke AP, et al. WHO Classification of Tumours of the Lung, Pleura, Thymus and Heart Lyon, France: International Agency for Research on Cancer; 2015.

3. Derks JL, Hendriks LE, Buikhuisen WA, et al. Clinical features of large cell neuroendocrine carcinoma: a population-based overview. Eur Respir J 2016;47:615-624.

4. Takei H, Asamura H, Maeshima A, et al. Large cell neuroendocrine carcinoma of the lung: a clinicopathologic study of eighty-seven cases. J Thorac Cardiovasc Surg 2002;124:285-292.

5. Korse CM, Taal BG, van Velthuysen ML, et al. Incidence and survival of neuroendocrine tumours in the Netherlands according to histological grade: experience of two decades of cancer registry. Eur J Cancer 2013;49:1975-1983.

6. Varlotto JM, Medford-Davis LN, Recht A, et al. Should large cell neuroendocrine lung carcinoma be classified and treated as a small cell lung cancer or with other large cell carcinomas? J Thorac Oncol 2011;6:1050-1058.

7. Derks JL, Leblay N, Thunnissen E, et al. Molecular Subtypes of Pulmonary Large-cell Neuroendocrine Carcinoma Predict Chemotherapy Treatment Outcome. Clin Cancer Res 2018;24:33-42.

8. George J, Walter V, Peifer M, et al. Integrative genomic profiling of large-cell neuroendocrine carcinomas reveals distinct subtypes of high-grade neuroendocrine lung tumors. Nat Commun 2018;9:1048.

9. Rekhtman N, Pietanza MC, Hellmann MD, et al. Next-Generation Sequencing of Pulmonary Large Cell Neuroendocrine Carcinoma Reveals Small Cell Carcinoma-like and Non-Small Cell Carcinoma-like Subsets. Clin Cancer Res 2016;22:3618-3629.

10. Zhou F, Hou L, Ding T, et al. Distinct clinicopathologic features, genomic characteristics and survival of central and peripheral pulmonary large cell neuroendocrine carcinoma: From different origin cells? Lung Cancer 2018;116:30-37.

11. Grondahl V, Binderup T, Langer SW, et al. Characteristics of 252 patients with bronchopulmonary neuroendocrine tumours treated at the Copenhagen NET Centre of Excellence. Lung Cancer 2019;132:141-149.

12. Cao L, Li ZW, Wang M, et al. Clinicopathological characteristics, treatment and survival of pulmonary large cell neuroendocrine carcinoma: a SEER population-based study. PeerJ 2019;7:e6539.

13. Asamura $\mathrm{H}$, Kameya $\mathrm{T}$, Matsuno $\mathrm{Y}$, et al. Neuroendocrine neoplasms of the lung: a prognostic spectrum. J Clin Oncol 2006;24:70-76.

14. Jiang $Y$, Lei $C$, Zhang $X$, et al. Double-edged role of radiotherapy in patients with pulmonary large-cell neuroendocrine carcinoma. J Cancer 2019;10:6422-6430.

15. Wakeam E, Adibfar A, Stokes S, et al. Defining the role of adjuvant therapy for early-stage large cell neuroendocrine carcinoma. J Thorac Cardiovasc Surg 2019.

16. Ogawa $\mathrm{H}$, Tanaka $\mathrm{Y}$, Kitamura $\mathrm{Y}$, et al. Efficacy of perioperative chemotherapy for pulmonary high-grade neuroendocrine carcinomas: a propensity score matching analysis. J Thorac Dis 2019;11:1145-1154.

17. Tsoukalas N, Baxevanos P, Aravantinou-Fatorou E, et al. Advances on systemic treatment for lung neuroendocrine neoplasms. Ann Transl Med 2018;6:146.

18. Filosso PL, Guerrera F, Evangelista A, et al. Adjuvant chemotherapy for large-cell neuroendocrine lung carcinoma: results from the European Society for Thoracic Surgeons Lung Neuroendocrine Tumours Retrospective Database. Eur J Cardiothorac Surg 2017;52:339-345.

19. Iyoda A, Hiroshima K, Moriya Y, et al. Prospective study of adjuvant chemotherapy for pulmonary large cell neuroendocrine carcinoma. Ann Thorac Surg 2006;82:1802-1807.

20. Kenmotsu $\mathrm{H}, \mathrm{Niho} \mathrm{S}$, Ito $\mathrm{T}$, et al. A pilot study of adjuvant chemotherapy with irinotecan and cisplatin for completely resected high-grade pulmonary neuroendocrine carcinoma (large cell neuroendocrine carcinoma and small cell lung cancer). Lung Cancer 2014;84:254-258. 
21. Rossi G, Cavazza A, Marchioni A, et al. Role of chemotherapy and the receptor tyrosine kinases KIT, PDGFRalpha, PDGFRbeta, and Met in large-cell neuroendocrine carcinoma of the lung. J Clin Oncol 2005;23:8774-8785.

22. Hanna N, Johnson D, Temin S, et al. Systemic Therapy for Stage IV Non-Small-Cell Lung Cancer: American Society of Clinical Oncology Clinical Practice Guideline Update. J Clin Oncol 2017;35:34843515 . 
Valorization 



\section{Valorization}

Worldwide, healthcare costs are increasing due to rising life expectancy and enhanced possibilities for treatment of various diseases. In the Netherlands, total costs for care and welfare increased from $€ 39$ billion in 1998, to $€ 100$ billion in $2018 .^{1}$ In this time period, costs for care in general hospitals and university medical centers increased from $€ 9$ billion to $€ 23.5$ billion. ${ }^{1}$ The challenge for the future is to further increase quality of healthcare with no or only minimal additional costs. One solution for this challenge is personalized medicine, with the purpose to provide the right medication at the right time to the right patient at minimal cost.

In the last decades, personalized medicine has evolved for different types of cancer, e.g. non-small cell lung carcinoma (NSCLC). In a group of patients with NSCLC selected by mutational analysis, targeted therapy with tyrosine kinase inhibitors (TKIs) has been registered as first line therapy. This personalized approach, in combination with application of immunotherapy (programmed death (ligand) 1 (Pd-(I)1) inhibition) in other patients, resulted in remarkably improved survival and even long-term 5-year survival in part of stage IV patients, compared to previous treatment with palliative chemotherapy. Selection of patients by mutational analysis and immunohistochemical markers for targeted- and immunotherapy, respectively, results in the most efficient treatment for patients, while at the same time the usage of this rather expensive medication is reduced in patients who will probably not respond to the treatment. ${ }^{2}$

Large cell neuroendocrine carcinoma (LCNEC) is part of the group of 'rare cancers', since it occurs in less than 6 out of 100,000 people each year. Despite the scarcity of each rare cancer, all rare cancers together comprise 1 out of 5 of all cancer cases in the Netherlands. Therefore, additional research to improve quality of life and survival in this special group of patients is necessary and this has also been recognized by patient organizations and grant providers. ${ }^{3}$ However, due to low patient numbers for each disease, proper research can be challenging. Furthermore, available resources are limited, since expensive research for all those rare cancer types would add up to extremely high costs for society. For these 'rare cancers' as LCNEC, personalized medicine could also be the solution to improve quality of life and survival at limited costs. In this thesis, different methods were used to evolve personalized medicine of LCNEC: expanding knowledge of LCNEC oncogenesis, transfer of knowledge from other tumor types, use of existing data and identification of clinically relevant subtypes. 


\section{Expanding basic knowledge of LCNEC oncogenesis}

Basic knowledge on oncogenesis is essential for development of personalized medicine. Insight in driver gene mutations, the role in tumor cell development and progression, and incidence of those mutations in patients might help to select pharmacological targets. For example, treatment with TKIs in NSCLC are based on driver mutations identified in EGFR, among others. ${ }^{4}$ This basic knowledge might also be helpful in development of predictive markers. By selecting patients who likely will respond to the therapy and only treating those patients, unnecessary side effects and costs will be prevented. For example, immunohistochemical staining of Pd-I1 expression is used as a predictive marker for $\mathrm{Pd}-(\mathrm{I}) 1$ therapy, but accuracy seems to be limited. Other markers and combinations of markers have been postulated in the past years, including $\mathrm{Cd} 8$ expression of tumor infiltrating cells, tumor mutational burden and imaging techniques. ${ }^{5-8}$ However, so far the value of all investigated markers is limited. More specific for rare cancer types, basic knowledge on oncogenesis might be helpful to compare the rare cancer to more prevalent cancer types. In chapter $\mathbf{3}$ of this thesis for example, augmented insight in oncogenesis of LCNEC is provided by an in-dept analysis of combined tumors with both an adenocarcinoma and LCNEC part.

\section{Transfer of knowledge from other tumor types}

An efficient way to develop new therapeutic strategies, is to use knowledge from research readily available from other types of cancer with comparable clinical characteristics and/or mechanisms of oncogenesis. Much of the time and costs in drug development are spent in the pre-clinical phase and clinical phase I studies. Those steps might be (partly) circumvented by transferring the knowledge to another tumor type, in which phase 2 and/or phase 3 studies might be initiated immediately. For example, much research on immunotherapy has been performed in melanoma with ipilimumab (a CTLA-4 blocking antibody), the first immune checkpoint inhibitor drug being FDA approved. ${ }^{9,10}$ Afterwards, knowledge could be transferred among others to renal cell carcinoma and NSCLC, resulting in different types of immunotherapy with FDA approval nowadays and research going on in various other tumor types.

By transferring the knowledge obtained in more prevalent types of cancer, information about applicability and effectiveness in a rare cancer type could be obtained with a limited number of patients and relatively low costs. We applied this method in chapters 7 and 8. In chapter 8, it was shown that Pd-I1 expression in stage IV LCNEC is more comparable to expression in small cell lung carcinoma ( $\mathrm{SCLC}$ ) than to expression in NSCLC. Although Pd-I1 expression seems to have limited predictive value in other studies, the low frequency of Pd-I1 positivity argue for LCNEC being an immune 
excluded tumor. In concordance with SCLC, the majority of those tumors may respond to combination therapy including Pd-(I)1 targeted therapy, but unlikely to monotherapy alone as is seen in NSCLC. Therefore, studies investigating monotherapy should not be performed and only combination therapy should be further explored for LCNEC. In chapter 7, The frequency of delta like ligand 3 (DII3) expression (a potential target for therapy in neuroendocrine carcinoma) in stage IV LCNEC tumors proved to be comparable to SCLC. Since more patients present with SCLC than with LCNEC, it might be easier to develop novel drugs for and execute phase I, II and III studies in SCLC patients. Based on comparable marker expression, medication specifically targeting DII3 that turns out to be effective in SCLC might also be effective in LCNEC. As proposed in chapter 10, information on clinically relevant subtypes in SCLC could be used to develop a hypothesis about additional relevant LCNEC subtypes. For example, since YAP1 and POU2F3 subtypes most likely are very small subgroups, obtained information on treatment efficacy in those SCLC patient groups might also be useful to aid treatment decisions in LCNEC patients.

\section{Efficient use of existing data}

Retrospective studies using data available from clinical practice may also be an efficient way to obtain additional insight in rare cancers. Examples of such databases are the United States Surveillance, Epidemiology, and End Results (SEER) program and the Netherlands Cancer Registry (NCR) database, both comprising the majority of newly diagnosed cancer patients in their respective countries. Besides epidemiological purposes, researches may request data on a specific tumor of interest to retrospectively answer research questions. Such a nationwide database is especially valuable in rare cancers, since it is the only way to obtain information on relatively large amounts of patients. A disadvantage of these nationwide registries is the limited amount of data registered for each patient. Smaller, local, retrospective studies could overcome this problem by obtaining more specific, additional information. In chapter $\mathbf{2}$, a retrospective study was executed with routinely performed CT-scans to obtain more information on molecular SCLC-like vs. NSCLC-like LCNEC. Unfortunately, it was not possible to discriminate between the two subtypes based on clinical interpretation, semantic features or a radiomics signature in this study. With improving techniques it might be possible to use radiomics to replace pathological investigations in the future. This could result in reduced inconvenience for the patient if tissue for histopathological examination has to be taken less often. Retrospective data was also used in chapter 9 to obtain information on metastatic patterns in neuroendocrine neoplasms of different primary origins. This study revealed that screening for brain metastases might be useful 
in patients with pulmonary neuroendocrine neoplasms, but not in patients with other neuroendocrine neoplasms. This insight could result in more personalized advice for screening of metastases, and might result in cost reduction through a reduction of unnecessary screening while preventing withholding of screening for patients who might benefit from it. The knowledge obtained in such a retrospective study could be used to initiate more focused, prospective trials to confirm the results.

\section{Identification of clinically relevant subtypes}

Identification of clinically relevant tumor subtypes can also benefit personalized medicine. In prevalent tumor types with homogeneous morphological characteristics, e.g. colorectal cancer, all patients used to be treated in a comparable way. However, in the last decades clinically relevant subtypes have been identified and as a result diagnostic processes and/or therapeutic regimens has been adopted. For example, in colorectal cancer microsatellite instability (MSI) and immunohistochemistry to reveal loss of mismatch repair (MMR) proteins are only determined in subgroups of patients (e.g. young age), since the chance of a MSI-high tumor is very low in patients outside these subgroups. ${ }^{11}$

Although only a limited number of patients present with a rare disease like LCNEC yearly, it has to be recognized that not all patients should be treated with the same regimen. In chapters $\mathbf{4}$ and $\mathbf{5}$ was shown that within LCNEC clinical subtypes of patients might exist, who may benefit from a different treatment approach. A subtype of patients with a solitary brain metastasis and Ki-67 proliferation index $\leq 40 \%$ showed prolonged overall survival compared to stage IV patients in general (chapter 4). Therefore, this group might benefit from more aggressive and even definitive treatment instead of 'standard' palliative chemotherapy. Another subtype with prolonged survival compared to general LCNEC was identified in chapter $\mathbf{5}$. This group consists of LCNEC patients with well differentiated morphology and preserved $\mathrm{pRb}$ immunostaining. Patients with these characteristics might benefit from treatment as applied to high-grade neuroendocrine tumors, instead of a neuroendocrine carcinoma regimen. Further research is needed to determine clinical relevance.

\section{Future perspective}

In the future, personalized medicine should be further developed for all types of cancer to increase survival rates and quality of life, and at the same time prevent unreasonable additional healthcare costs. More specifically, in LCNEC, more research should be performed to confirm the clinical relevance of the molecular LCNEC subtypes, clinical subtypes and predictive and prognostic markers described in this thesis. Furthermore, 
personalized medicine for LCNEC could be improved in several other ways to optimize quality of healthcare while limiting additional healthcare costs. For example, a more reliable diagnosis based on only small biopsy material would prevent the necessity of re-biopsies and even unnecessary resections. Among others, this diagnosis could be improved by application of new immunohistochemical markers, e.g. pRb. Furthermore, new techniques as 'proteomics', 'histomics' or 'liquid biopsies' might be used in the future to obtain important information with only a limited amount of tumor tissue. ${ }^{12-14}$ These approaches might be able to further differentiate known molecular subtypes of LCNEC, but they might also reveal new relevant subtypes. Another promising method for personalized medicine is the use of 'organoids'. With organoids, a threedimensional in vitro model of the tumor is created, derived from tissue specific stem cells, e.g. cancer cells. High throughput screening of medication on these organoids might reveal new targeted therapies for subtypes of the disease. Furthermore, knock-in or knock-out of specific genes in organoids could augment the knowledge on oncogenesis of a rare disease like LCNEC. ${ }^{15}$

\section{Summary}

This thesis contributes to more personalized medicine of LCNEC by analyzing potential prognostic and predictive markers, identifying possible clinically relevant subtypes and using existing data to give recommendations for diagnostic work-up. In the future, more focus on personalized medicine is necessary for all cancer types, and specifically for rare diseases such as LCNEC, to improve outcome with no or only limited additional healthcare costs. 


\section{References}

1. CBS. Zorguitgaven; zorgaanbieders en financiering. 21-06-2019. Available at https://opendata.cbs.nl/ statline/\#/CBS/nl/dataset/84053ned/table?ts=1584634257241. Accessed 19-3-2020

2. van den Broek D, Hiltermann TJN, Biesma B, et al. Implementation of Novel Molecular Biomarkers for Non-small Cell Lung Cancer in the Netherlands: How to Deal With Increasing Complexity. Front Oncol 2019;9:1521.

3. KWF. Onderzoek naar zeldzame kanker. Available at https://www.kwf.nl/kanker/zeldzame-kanker/ onderzoek-naar-zeldzame-kanker. Accessed 20-3-2020

4. Planchard D, Popat S, Kerr K, et al. Metastatic non-small cell lung cancer: ESMO Clinical Practice Guidelines for diagnosis, treatment and follow-up. Ann Oncol 2018;29:iv192-iv237.

5. Niemeijer AN, Sahba S, Smit EF, et al. Association of tumour and stroma PD-1, PD-L1, CD3, CD4 and CD8 expression with DCB and OS to nivolumab treatment in NSCLC patients pre-treated with chemotherapy. British journal of cancer 2020.

6. Bensch F, van der Veen EL, Lub-de Hooge MN, et al. (89)Zr-atezolizumab imaging as a non-invasive approach to assess clinical response to PD-L1 blockade in cancer. Nature medicine 2018;24:1852-1858.

7. Krieger T, Pearson I, Bell J, et al. Targeted literature review on use of tumor mutational burden status and programmed cell death ligand 1 expression to predict outcomes of checkpoint inhibitor treatment. Diagn Pathol 2020;15:6.

8. Fumet JD, Truntzer C, Yarchoan M, et al. Tumour mutational burden as a biomarker for immunotherapy: Current data and emerging concepts. Eur J Cancer 2020;131:40-50.

9. BMS. FDA Approves Yervoy. 28-03-2011. Available at https://www.drugs.com/nda/ipilimumab_ 100818.html. Accessed 14-4-2020

10. Atkins MB, Clark JI, Quinn DI. Immune checkpoint inhibitors in advanced renal cell carcinoma: experience to date and future directions. Ann Oncol 2017;28:1484-1494.

11. FMS. Richtlijn colorectaal carcinoom. Available at https://www.oncoline.nl/colorectaalcarcinoom. Accessed 14-4-2020

12. Gonzalez D, Dietz RL, Pantanowitz L. Feasibility of a Deep Learning Algorithm to Distinguish Large Cell Neuroendocrine from Small Cell Lung Carcinoma in Cytology Specimens. Cytopathology : official journal of the British Society for Clinical Cytology 2020.

13. Belczacka I, Latosinska A, Metzger J, et al. Proteomics biomarkers for solid tumors: Current status and future prospects. Mass Spectrom Rev 2018.

14. Esposito Abate R, Frezzetti D, Maiello MR, et al. Next Generation Sequencing-Based Profiling of Cell Free DNA in Patients with Advanced Non-Small Cell Lung Cancer: Advantages and Pitfalls. Cancers 2020;12.

15. Drost J, van Boxtel R, Blokzijl F, et al. Use of CRISPR-modified human stem cell organoids to study the origin of mutational signatures in cancer. Science 2017;358:234-238. 
Dankwoord 



\section{Dankwoord}

Het is gelukt! Hoewel een PhD soms best een eenzaam traject is, sta je er nooit echt alleen voor. Ik heb mij de afgelopen jaren dan ook gelukkig mogen prijzen met mijn promotieteam, collega's, vrienden en familie. Via deze weg wil ik graag iedereen bedanken die op welke manier dan ook heeft bijgedragen aan het tot stand komen van dit boekje.

Allereerst wil ik natuurlijk mijn (co)promotoren bedanken, die het mogelijk hebben gemaakt om dit PhD-traject snel tot een goed einde te brengen. Anne-Marie, het vertrouwen en de vrijheid die je mij hebt gegeven om zelf met ideeën te komen heb ik enorm gewaardeerd en hebben mij gestimuleerd om mezelf te ontwikkelen als onderzoeker. Altijd had jij ideeën hoe we de data in nog mooiere figuren weer konden geven. Heel veel dank daarvoor!

Ernst-Jan, de wereld van de pathologie en moleculaire biologie kreeg ik als clinicus cadeau bij dit promotietraject... Ik heb enorm veel geleerd van de discussies over mutatie-analyses, kleuringen en moleculaire processen; kennis en ervaring waar ik de rest van mijn carrière plezier van ga hebben. Dankjewel voor de introductie in deze 'nieuwe' wereld!

Jules, mijn PhD-traject begon als een rijdende trein door alle projectjes die jij nog voor mij had achtergelaten. Je eindeloze nieuwe ideeën 'waar we ook eens naar zouden moeten kijken' maakten mij soms moedeloos, maar waren uiteindelijk steeds weer inspirerend. Dankjewel voor al je hulp en enthousiasme, ik weet zeker dat er nog een hele mooie toekomst in het onderzoek voor jou aan zit te komen!

Gelukkig zetten Laura en Nicole het onderzoek naar carcinoïden en LCNEC nog door, er zijn nog zoveel vragen te beantwoorden. Laura, je bent mijn held! Dank voor alle kleuringen die je 'even' voor mij hebt gedaan en de dummy uitleg als ik weer eens iets niet snapte op het lab. Ik heb er alle vertrouwen in dat je de PREDICT-studie tot een goed einde gaat brengen en al die andere projecten die je 'erbij' mag doen ook natuurlijk. Nicole, leuk om te zien dat jij de LCNEC projecten hebt overgenomen en met veel enthousiasme aan de slag bent gegaan! Heel veel succes de komende jaren met het KWF-project en natuurlijk het voortzetten van de andere projecten.

Furthermore, I would like to thank the assessment committee for the time to read and assess my thesis. Thank you, Prof. dr. A. Zur Hausen, Prof. dr. V. Tjan-Heijnen, Prof. dr. M. Vooijs, Prof. dr. F. Blackhall and Prof. dr. M. Volante. 
Lisa, in de afgelopen jaren werd jij steeds meer bij het 'pul NEN' onderzoeksteam betrokken. Dankjewel voor al je tijd om coupes te kijken, maar bovenal het enthousiasme, optimisme en de levenslessen tussendoor ('niet aan kinderen beginnen tijdens je opleiding...'). Jan, vanuit Rotterdam ben jij inmiddels ook betrokken bij de LCNEC studies. Ook jij was altijd enthousiast, zelfs als ik met twee enorme dozen aan kwam zetten als we alleen 'een paar coupes' zouden kijken. Dank voor de hulp!

Het team van de moleculaire diagnostiek heeft mij veel geholpen bij het labwerk en was altijd bereid om tussendoor coupes te snijden of te kleuren. Dankjewel voor alle hulp bij de optimalisatie van antilichamen Cécile, en ook veel dank natuurlijk aan Guido, Stefan, Erik, Carla en Andrea.

Graag wil ik ook iedereen bedanken die als co-auteur een waardevolle bijdrage heeft geleverd aan het onderzoek en de manuscripten. Sebastian en Hester, speciale dank voor jullie input tijdens het onderzoek naar imaging features bij LCNEC, dat was zonder jullie hulp nooit gelukt! Judith en Wouter, ondanks dit mooie uitstapje bij de longziekten, blijf ik toch bij mijn keuze voor de interne geneeskunde en medische oncologie. Ik vond het daarom ook erg leuk om de kennis over long- en gastrointestinale tumoren te kunnen combineren. Onze database is nog lang niet 'af', dus de komende jaren zullen we zeker nog samenwerken.

In de afgelopen jaren heb ik verschillende studenten mogen begeleiden bij (afstudeer)stages. Ik hoop dat ik jullie allemaal heb kunnen laten zien dat er echt hele leuke kanten aan onderzoek zitten (en dat SPSS niet eng is), ook al hebben jullie helaas ook kennis mogen maken met de tegenslagen die met onderzoek gepaard gaan. Mariya, Cleo, Selin en Romy, ik vond het leuk om jullie wegwijs te maken in het leven van een onderzoeker. Aniek en Simone, jullie ook bedankt voor de hulp bij het invullen van de MMC-MUMC database!

Karin, in het eerste jaar waren wij kamergenoten en heb ik kunnen profiteren van alle tips en tricks die jij als ervaren PhD'er met mij deelde, dankjewel! Gelukkig is jouw traject nu ook echt afgerond. Justine, we were roommates in the second year of my $\mathrm{PhD}$ and the topics of our projects couldn't be further apart... However, I really enjoyed the talks about sports, (healthy) food and the presentation course we did together. Good luck finishing your thesis!

Natuurlijk wil ik ook graag mijn collega's van de pulmonologie-gang bedanken. De lunches, borrels (helaas aan het einde alleen nog virtueel...), (lekkere) koffies en 
gewoon praatjes tussendoor als iets even tegen- of juist meezat, waren heel waardevol! Daarom: Anita, Harry, Juanita, Juliette, Lieke, Lisanne, Marco, Martijn, Mieke, Niki, Pieter, Rianne, Rosanne, Ramon, Sara, Sarah, Sophie, Vasili, Viviënne, Wessel en Wouter, bedankt! Tijdens de laatste fase van dit promotietraject werkte ik weer in de kliniek. Alle internisten en arts-assistenten interne van het Máxima Medisch Centrum, dank voor de support bij de laatste loodjes!

Lieve Que Pasies. Al 10 jaar gaan we als 'Que Pasa' door het leven, en de vriendschap met sommigen van jullie gaat nog veel langer terug. Wat begon met samen hard roeien, is uitgegroeid tot een hechte vriendengroep. Dank jullie wel voor alle Friday Night Dinners, weekendjes weg, de sinterklaasgedichten en het altijd bereid zijn om mijn geklaag aan te horen. Met dr. Lonneke en (bijna) dr. Lisanne had ik mooie PhD voorbeelden en Nine, ik vind het super dat jij het promotie-avontuur inmiddels ook bent aangegaan (ondanks al onze verhalen...). Karlijn, het klopt niet dat jij dit proces niet mee hebt kunnen maken. Je was altijd mijn grote voorbeeld op academisch gebied en bent ook in de afgelopen jaren een enorme motivatie en inspiratie geweest om door te gaan en iets moois van mijn onderzoek te maken.

Een gezonde geest in een gezond lichaam... De broodnodige afleiding kwam vooral van het sporten. Veel dank aan de Roze Pekskes voor vele uren in de boot, koffie drinken achteraf én alle mooie overwinningen! Het dames-Triotters team heeft mij steeds gemotiveerd om te blijven trainen voor en plezier te houden in de triathlon. Dank voor de gezelligheid, de leuke wedstrijden en het uitwisselen van alle tips \& tricks. Er was een corona-crisis en zwangerschap voor nodig om mij aan het Crossfitten te krijgen, maar gelukkig voelde ik mij meer dan welkom bij Youact! Pim, Marjan, Aagje en Nathan: speciale dank aan jullie voor alle steun en gezelligheid en op naar nog heel veel Geldropse bbq's, trainingen en spelletjesavonden(?).

Lieve papa en mama, van jongs af aan hebben jullie mij gestimuleerd om mijn talenten te benutten. Nooit door te pushen, wel door interesse te tonen, vertrouwen te hebben en af te remmen waar nodig. Ook nu ik op eigen benen sta, blijft dat afremmen soms nodig, en dat hebben jullie op de momenten dat het moest dan ook subtiel of minder subtiel gedaan. Maar bovenal was er altijd interesse voor mijn onderzoek én voor alle dingen die daarnaast speelden. Dank voor de stevige basis en voor alle momenten samen, de steun en liefde in de afgelopen jaren. Lieve zus en broertje, wat zijn we verschillend en toch ook hetzelfde, ik zou jullie voor geen goud willen missen! Margriet, de onderzoekswereld heb jij tijdens je master kunnen verkennen en daarna heb je heel verstandig besloten een hele andere kant op te gaan. Ook al woon je (te) ver weg, we 
houden contact en je blijft mijn grote kleine zus. Joris, als ik mezelf door jouw ogen zou moeten beschrijven, denk ik aan 'nerd, burger en hipster'. Gelukkig ben je zelf stiekem ook een nerd en hebben jij en Vita inmiddels de nodige burgerpunten verzameld. Nog even doorzetten, dan hebben jullie die master straks ook binnen! Het fijne aan een oma is dat ze trots op je is, wat je ook doet. Dankjewel oma, voor je eindeloze vertrouwen! Natuurlijk wil ik ook mijn schoonfamilie, Ilse, Werner, Eric, Geke \& Robin bedanken voor alle leuke etentjes, logeerpartijen en de interesse die jullie getoond hebben voor mijn onbegrijpelijke onderzoek.

Lieve Annick, wat had ik zonder jou moeten beginnen? Inhoudelijk begreep je lang niet altijd waar ik mee bezig was, ook al deed je echt je best om te onthouden waar de projecten $1 \mathrm{t} / \mathrm{m} 10$ over gingen. Maar je was er altijd, om de kleine succesjes te vieren, maar ook als het minder goed ging. Dank voor al je relativerende en opbeurende woorden ('met elke afwijzing ben je weer een stapje dichter bij succes') en al die keren dat je me uit de put kon halen als ik dacht de slechtste PhD-student op de wereld te zijn. En meer nog dankjewel voor al die leuke momenten in de afgelopen jaren en het meest fantastische huwelijksaanzoek ooit. Het laatste deel van het onderzoek had ik vrijwel continue gezelschap van ons kleine wondertje Turi. Niet zo praktisch... maar wel heerlijk! Ik hou van jullie en heb nu al zin in alle avonturen die we nog samen gaan beleven! 
List of publications 



\section{List of publications}

\section{Original papers (peer reviewed)}

- $\quad$ E.B. Burger, B.C.M. Hermans, D.M.C.B. van Zeeben-van der Aa, Netwerkrichtlijn kindermishandeling en huiselijk geweld in Limburg; Aanpak binnen de acute zorgketen. Tijdschrift voor Kindergeneeskunde. 2014;2;64-9.

- B.C.M. Hermans, L.C.G.G. Persoon, F.J.P. Hoebers, F. Verhaegen, E.G.C. Troost. Weekly kilovoltage cone beam computed tomography for detection of dose discrepancies during (chemo)radiotherapy for head and neck cancer. Acta Onco. 2015;54(9):1483-9.

- B.C.M. Hermans, J.L. Derks, E. Thunnissen, R.J. van Suylen, M.A. den Bakker, H.J.M. Groen, E.F. Smit, R.A. Damhuis, E.C. van den Broek, C.M. Stallinga, G.M. Roemen, E.J.M. Speel, A.-M.C. Dingemans, Prevalence and prognostic value of PD-L1 expression in molecular subtypes of metastatic large cell neuroendocrine carcinoma (LCNEC). Lung Cancer. 2019;130:179-86.

- B.C.M. Hermans, J. Derks, H.J.M. Groen, J.A. Stigt, R.J. van Suylen, L.M. Hillen, E.C. van den Broek, E.M. Speel, A.C. Dingemans, Large cell neuroendocrine carcinoma with a solitary brain metastasis and low Ki-67: a unique subtype, Endocr Connect. 2019;8(12):1600-1606.

- B.C.M. Hermans, J.L. Derks, E. Thunnissen, R.J. van Suylen, M.A. den Bakker, H.J.M. Groen, E.F. Smit, R.A. Damhuis, E.C. van den Broek, P. group, A. Ruland, E.J.M. Speel, A.M.C. Dingemans, DLL3 expression in large cell neuroendocrine carcinoma (LCNEC) and association with molecular subtypes and neuroendocrine profile, Lung Cancer. 2019;138:102-108.

- B.C.M. Hermans, S. Sanduleanu, J.L. Derks, H. Woodruff, L. Hillen, R. Casale, F. Mohamed Hoesein, E. de Jong, D.M.H.J. ten Berge, E.J.M. Speel, P. Lambin, H.A. Gietema, A-M.C. Dingemans, Exploring imaging features of molecular subtypes of large cell neuroendocrine carcinoma (LCNEC), Lung Cancer. 2020;148:94-99. 
- B.C.M. Hermans, J.L. Derks, L. Moonen, C.H.J. Habraken, J. von der Thüsen, L.M. Hillen, E.J.M. Speel, A-M.C. Dingemans, Pulmonary neuroendocrine neoplasms with well differentiated morphology and high proliferative activity: illustrated by a case series and review of the literature, Lung Cancer. 2020;150:152-158.

- B.C.M. Hermans, J. de Vos-Geelen, J.L. Derks, L. Latten, I.H. Liem, J.M. van der Zwan, E.J.M. Speel, M.W. Dercksen, A-M.C. Dingemans, Unique metastatic patterns in neuroendocrine neoplasms of different primary origin, Neuroendocrinology. 2020 Nov 23. Online ahead of print.

- L. Moonen, J.L. Derks, B.C.M. Hermans, I.M. Bunnik, L.M. Hillen, R.J. van Suylen, M.A. den Bakker, J.H. von der Thüsen, R.A. Damhuis, E.C. van den Broek, W.A. Buikhuisen, A-M.C. Dingemans, E.J.M. Speel, Pre-operative biopsy diagnosis in pulmonary carcinoids, a shot in the dark, J Thorac Oncol. 2021 Apr;16(4):610-618.

\section{Letter to the editor (peer reviewed)}

- B.C.M. Hermans, M.M. Zanders, N.H.M. van Oostrum, P.H.M. Kuijper, L. Nieuwenhuizen, Successful treatment of aplastic anaemia with cyclosporine during pregnancy, Neth J Med. 2019 Apr;77(3):126-127.

\section{Review articles (peer reviewed)}

- $\quad$ L.E. Hendriks, B.C.M. Hermans, M.H.J. van den Beuken-van Everdingen, M.M.H. Hochstenbag, A.M. Dingemans. Effect of bisphosphonates, denosumab and radioisotopes on bone pain and quality of life in non-small cell lung cancer patients with bone metastases: a systematic review. J Thorac Oncol. 2016 Feb;11(2):155-73.

- B.C.M. Hermans, J.L. Derks, L.E.L. Hendriks, L. Moonen, E.J.M. Speel, A-M.C. Dingemans. Delta Like Ligand 3 (DLL3) als doelwit voor gerichte behandeling van het kleincellig longcarcinoom (SCLC) en het pulmonaal grootcellig neuro-endocrien carcinoom (LCNEC) (in Dutch). Ned Tijdschr Oncol. 2019;16:325-33. 
Curriculum Vitae 



\section{Curriculum Vitae}

Bregtje Hermans was born in Gouda, the Netherlands, on March 22th, 1989. In 2007, she graduated cum laude from the Coornhert Gymnasium (Gouda, the Netherlands) and started her studies on Medical Physical Sciences at the Free University (Amsterdam, the Netherlands). She graduated cum laude for her Bachelor in 2011 and was accepted to the Master Physician - Clinical Investigator at Maastricht University (Maastricht, the Netherlands). During her Master, she performed research projects at the Department of Pulmonology (supervisor prof. A-M.C. Dingemans) and the Department of Radiotherapy (supervisor dr. E.G.C. Troost) at

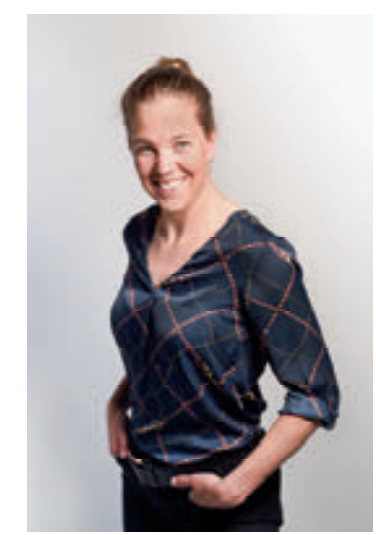
Maastricht University Medical Centre+ (MUMC+). She obtained her Master Degree (cum laude) in 2015 and started working as a resident Internal Medicine at Máxima Medical Centre (Eindhoven/Veldhoven, the Netherlands). She completed the first 2 years of training to become a specialist for Internal Medicine from 2016 to 2018.

Bregtje started her PhD project in 2018 under the supervision of prof. dr. A-M.C. Dingemans, prof. dr. E-J.M. Speel and dr. J.L. Derks at MUMC+. She focused on pathological, radiological and clinical characteristics of pulmonary large cell neuroendocrine carcinoma. She was honored with travel grants to attend the European Society of Medical Oncology (ESMO) conference in 2018, the European Thoracic Oncology Platform (ETOP) residential workshop in 2018, the European Neuroendocrine Tumor Society (ENETS) conference in 2019 and the World Conference on Lung Cancer (WCLC) in 2019. Currently, Bregtje is working as a resident Internal Medicine at Máxima Medical Centre and she is planning to continue her training with the subspecialty Medical Oncology at MUMC+. 


